UNIVERSITE DU QUEBEC A CHICOUTIMI

\title{
STRATIGRAPHIE ET SIGNIFICATIONS DES MINERALISATIONS \\ DANS LE SECTEUR DU MONT BOURBEAU, CANTON DE MCKENZIE, CHIBOUGAMAU
}

\author{
Par \\ PIERRE PILOTE
}

B. Sc. A. (Gểnie Géologique)

\author{
MEMOIRE PRESENTE EN VUE DE L'OBTENTION \\ DE LA MAITRISE EN SCIENCES \\ DE LA TERRE
}

MAI 1986 


\section{Bibliothèque}

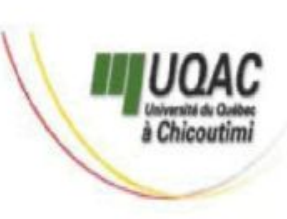

\section{Mise en garde/Advice}

Afin de rendre accessible au plus Motivated by a desire to make the grand nombre le résultat des results of its graduate students' travaux de recherche menés par ses research accessible to all, and in étudiants gradués et dans l'esprit des accordance with the rules règles qui régissent le dépôt et la governing the acceptation and diffusion des mémoires et thèses diffusion of dissertations and produits dans cette Institution, theses in this Institution, the I'Université du Québec à Université du Québec à Chicoutimi (UQAC) est fière de Chicoutimi (UQAC) is proud to rendre accessible une version make a complete version of this complète et gratuite de cette œuvre. work available at no cost to the reader.

L'auteur conserve néanmoins la The author retains ownership of the propriété du droit d'auteur qui copyright of this dissertation or protège ce mémoire ou cette thèse. thesis. Neither the dissertation or Ni le mémoire ou la thèse ni des thesis, nor substantial extracts from extraits substantiels de ceux-ci ne it, may be printed or otherwise peuvent être imprimés ou autrement reproduced without the author's reproduits sans son autorisation. permission. 
$\underline{\text { RESUME }}$

Ce mémoire porte sur une région de $18 \mathrm{~km} 2$ centrée sur le mont Bourbeau, lequel est situé immédiatement au nord de la ville de Chibougamau. Les lithologies présentes sont les roches volcano-sédimentaires de la partie supérieure du Groupe de Roy soit les Formations de Gilman et de Blondeau, le Complexe de Cummings et le stock tonalitique du lac Line. Toutes les lithologies précédentes sont d’âge archéen. Le degré de métamorphisme atteint le faciès schiste vert. Des lambeaux protérozolques se retrouvent localement sur le mont Bourbeau et au sud du lac Larone.

De façon plus spécifique, la Formation de Blondeau est constituée de volcanoclastites de caractère distal. Des faciès plus proximaux se trouve sur le mont Bourbeau, ceci appuyant 1'hypothèse de la proximité d'un centre felsique localisé vers le nord, ou l'est.

$\mathrm{Ce}$ terrain est affecté par plusieurs failles et cisaillements d'orientations variées, associés à des réseaux de fractures d’âge spécifique: syn-volcanique (pré-tectonique), syn à tardi-tectonique et même protérozoìque.

Les indices minéralisés de type stratiforme se rencontrent le plus souvent dans la Formation de Blondeau et exceptionnellement dans la Formation de Gilman. Les indices de type filonien se présentent pour la plupart dans des fractures ou brèches à $1^{\prime}$ intérieur ou en bordure des différents filons-couches du Complexe de Cummings. Globalement, ce complexe apparait d'ailleurs dans ce secteur comme la cible la plus intéressante pour 1'exploration minérale.

Les indices stratiformes et filoniens visités ont des origines souvent distinctes. Leur distribution varie dans le temps et également

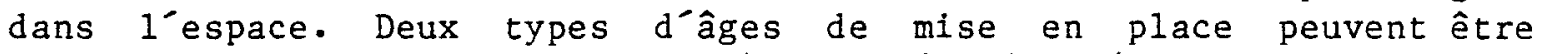
distingués chez ces indices: 1) pré-kénoréens (de types stratiforme et filonien), reliés à 1'activité volcanique et 2) syn à post-kénoréens (filoniens), de nature encore mal définie.

$C^{\prime}$ est sur le mont Bourbeau que $1^{\text {'on }}$ retrouve la plus grande variété d'indices minéralisés. La proximité d’un éventuel centre felsique dans les environs explique possiblement en partie cette situation.

Le stock tonalitique du lac Line, d'âge tardi-tectonique, contient ponctuellement des minéralisations en argent, molybdène et or. Les rôles qu’a pu jouer ce pluton comme réservoir de métaux ( $\mathrm{Au}$ et $\mathrm{Ag}$ ), comme source de chaleur potentielle pour une certaine part des fluides hydrothermaux et sur le style tectonique local restent à être évalués. 


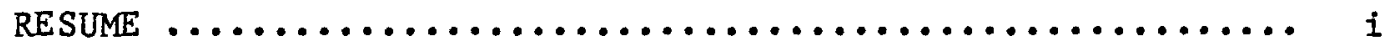

TABLES DES MATIERES $\ldots \ldots \ldots \ldots \ldots \ldots \ldots \ldots \ldots \ldots \ldots \ldots \ldots \ldots$ ii

LISTE DES FIGURES $\ldots \ldots \ldots \ldots \ldots \ldots \ldots \ldots \ldots \ldots \ldots \ldots \ldots \ldots \ldots$ v

LISTE DES TABLEAUX $\ldots \ldots \ldots \ldots \ldots \ldots \ldots \ldots \ldots \ldots \ldots \ldots \ldots \ldots$

LISTE DES PLANCHES $\ldots \ldots \ldots \ldots \ldots \ldots \ldots \ldots \ldots \ldots \ldots \ldots \ldots \ldots$

1. INTRODUCTION $\ldots \ldots \ldots \ldots \ldots \ldots \ldots \ldots \ldots \ldots \ldots \ldots \ldots \ldots \ldots \ldots \ldots$

1.1 Situation géographique $\ldots \ldots \ldots \ldots \ldots \ldots \ldots \ldots \ldots \ldots$

1.2 Buts du travail $\ldots \ldots \ldots \ldots \ldots \ldots \ldots \ldots \ldots \ldots \ldots \ldots \ldots$

1.3 Travaux antérieurs $\ldots \ldots \ldots \ldots \ldots \ldots \ldots \ldots \ldots \ldots \ldots$

1.4 Méthodes de travail $\ldots \ldots \ldots \ldots \ldots \ldots \ldots \ldots \ldots \ldots \ldots \ldots 4$

1.5 Remerciements $\ldots \ldots \ldots \ldots \ldots \ldots \ldots \ldots \ldots \ldots \ldots \ldots \ldots$

2. GEOLOGIE REGIONALE ......................... 7

2.1 Introduction $\ldots \ldots \ldots \ldots \ldots \ldots \ldots \ldots \ldots \ldots \ldots \ldots \ldots$

2.2 Généralités $\ldots \ldots \ldots \ldots \ldots \ldots \ldots \ldots \ldots \ldots \ldots \ldots \ldots$

2.3 Groupe de Roy $\ldots \ldots \ldots \ldots \ldots \ldots \ldots \ldots \ldots \ldots \ldots \ldots \ldots \ldots$

2.4 Groupe d'opémisca $\ldots \ldots \ldots \ldots \ldots \ldots \ldots \ldots \ldots \ldots \ldots \ldots$

2.5 Roches intrusives $\ldots \ldots \ldots \ldots \ldots \ldots \ldots \ldots \ldots \ldots \ldots$

2.6 Structure et métamorphisme $\ldots \ldots \ldots \ldots \ldots \ldots \ldots \ldots 18$

3. GEOLOGIE LOCALE $\ldots \ldots \ldots \ldots \ldots \ldots \ldots \ldots \ldots \ldots \ldots \ldots \ldots \ldots \ldots . \ldots . \ldots . \ldots$

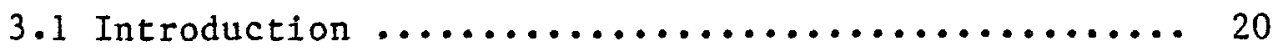


3.2 Groupe de Roy $\ldots \ldots \ldots \ldots \ldots \ldots \ldots \ldots \ldots \ldots \ldots \ldots \ldots \ldots . \ldots \ldots$

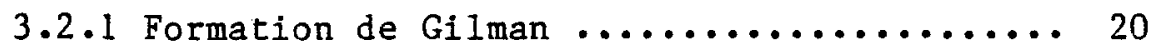

3.2 .2 Formation de Blondeau ................ 22

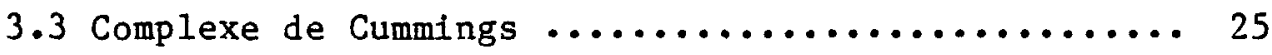

3.3.1 Filon-couche de Roberge ............. 25

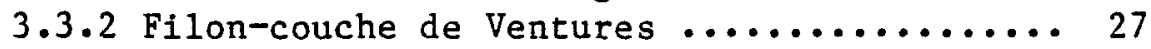

3.3.3 Filon-couche de Bourbeau .............. 29

3.4 Dykes $\ldots \ldots \ldots \ldots \ldots \ldots \ldots \ldots \ldots \ldots \ldots \ldots \ldots \ldots \ldots \ldots \ldots \ldots \ldots$

3.4.1 Dykes porphyriques en plagioclase ....... 31

3.4 .2 Dyke pyroxénitique ................ 32

3.5 Stock tonalitique du lac Line .............. 32

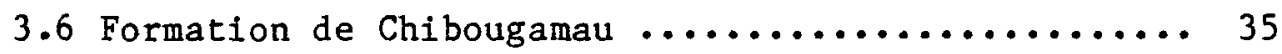

4. GEOLOGIE STRUCTURALE ....................... 37

4.1 P1is $\ldots \ldots \ldots \ldots \ldots \ldots \ldots \ldots \ldots \ldots \ldots \ldots \ldots \ldots \ldots \ldots . \ldots \ldots$

4.2 Failles $\ldots \ldots \ldots \ldots \ldots \ldots \ldots \ldots \ldots \ldots \ldots \ldots \ldots \ldots . \ldots \ldots$

4.2 .1 Introduction $\ldots \ldots \ldots \ldots \ldots \ldots \ldots \ldots \ldots \ldots . \ldots \ldots$

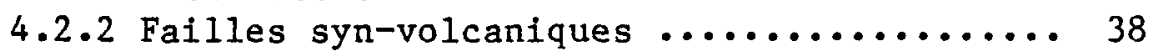

4.2 .3 Failles syn-tectoniques ............. 41

4.2.4 Ceinture de cisaillement du lac Gwillim ... 46

4.2 .5 Failles protérozoiques .............. 50

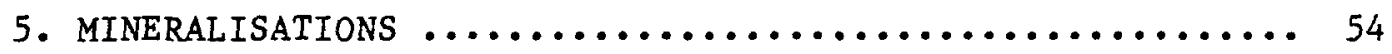

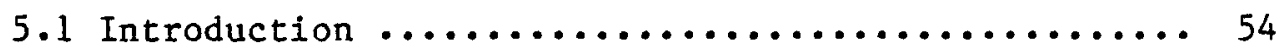

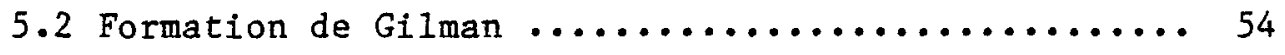

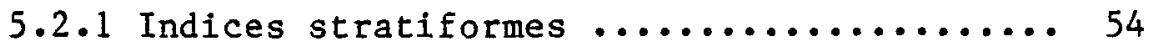

5.2 .2 Indice du lac Ham ................. 56

5.3 Formation de Blondeau $\ldots \ldots \ldots \ldots \ldots \ldots \ldots \ldots \ldots \ldots . \ldots . \ldots$

5.3.1 Indice des lacs Larone et Devilliers ..... 59 
5.4 Formation de Blondeau et bordures des

filons-couches du Complexe de Cummings .........66 63

5.4.1 Indices des dômes du radar .............6 63

$5.4 .2 \mathrm{~L}^{-}$indice $\mathrm{d}^{-}$argent $\ldots \ldots \ldots \ldots \ldots \ldots \ldots \ldots \ldots . \ldots 66$

5.4.3 Apophyse du filon-couche de Bourbeau ..... 68

5.4 .4 Sommet du filon-couche de Ventures ....... 71

5.5 Le Complexe de Cummings $\ldots \ldots \ldots \ldots \ldots \ldots \ldots \ldots \ldots \ldots$

5.5 .1 Le "Main Showing" .................. 72

5.5.2 Filons pyriteux, filon-couche de Ventures.. 74

5.5 .3 La zone "Bel-3" .................... 76

5.6 Le stock tonalitique du lac Line ............ 83

5.7 Protérozoĩque et Formation de Chibougamau ....... 84

6. DISCUSSION $\ldots \ldots \ldots \ldots \ldots \ldots \ldots \ldots \ldots \ldots \ldots \ldots \ldots \ldots \ldots, 86$

6.1 Introduction $\ldots \ldots \ldots \ldots \ldots \ldots \ldots \ldots \ldots \ldots \ldots \ldots . \ldots . \ldots . \ldots$

6.2 Minéralisations volcanogènes et leur mise

en place ............................... 86

6.3 Minéralisations filoniennes (tardi ou post-tectoniques) et leur mise en place ..........101

6.4 Considérations sur les modèles précédents et

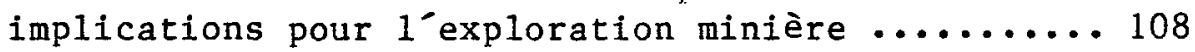

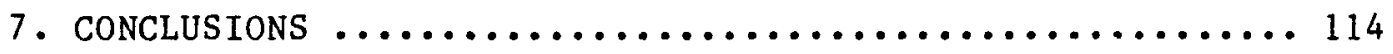

REFERENCES $\ldots \ldots \ldots \ldots \ldots \ldots \ldots \ldots \ldots \ldots \ldots \ldots \ldots \ldots \ldots \ldots \ldots \ldots$

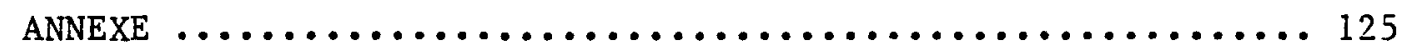

1 Planches, comprenant les photographies et les

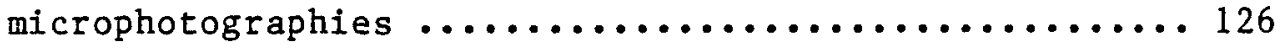

2 Analyses chimiques, éléments traces et minéraux

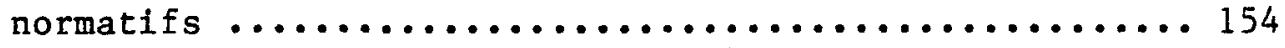

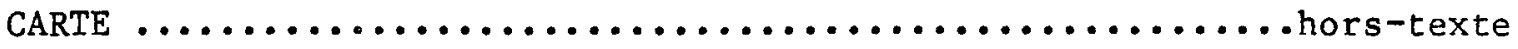

Stratigraphie de la région du mont Bourbeau ( $1: 5$ 000) 


\section{LISTE DES FIGURES}

page

FIGURE 1: Localisation du secteur étudié et géographie de la région de Chibougamau ................... 2

FIGURE 2: Localisation de la région de Chibougamau dans 1 a ceinture orogénique de $1^{\prime}$ Abitibi ............. 8

FIGURE 3: Géologie simplifiée de la région de Chibougamau • 10

FIGURE 4: Représentation schématique de la stratigraphie du camp minier de Chibougamau ............... 11

FIGURE 5: Coupe structurale de la partie ouest du mont Bourbeau .............................. 43

FIGURE 6: Distribution et répartition des failles et des cisaillements dans les environs de la ceinture de cisaillement du lac Gwillim .............. 47

FIGURE 7: Distribution et répartition des failles et des cisaillements dans la région du mont Bourbeau ... 48

FIGURE 8: Représentation schématique de 1 'indice minéralisé du lac Ham ................... 57

FIGURE 9: Indice du lac Devilliers, Formation de Blondeau . 60

FIGURE 10: Indices des Dômes du Radar, mont Bourbeau ...... 64

FIGURE 11: L'indice d'argent, à la base du filon-couche de Bourbeau et des volcaniclastites de la Formation de Blondeau ........................... 67

FIGURE 12: Schéma des indices se retrouvant dans les environs $d$ 'une apophyse de composition dunitique, filon-couche de Bourbeau, injectée dans les volcanoclastites de la Formation de Blondeau ... 69

FIGURE 13: Le "Main Showing", filon-couche de Roberge, mont Bourbeau ............................ 73

FIGURE 14: Coupe-type du filon-couche de Ventures et positions de filonets pyriteux, mont Bourbeau ... 75 
FIGURE 15: Cadre géologique de 1'indice "Be1-3" ......... 77

FIGURE 16: Section simplifiée de 1'indice "Be1-3" ........ 79

FIGURE 17: Plan simplifiée de la galerie d'exploration

"Be1-3" 


\section{LISTE DES TABLEAUX}

page

TABLEAU 1: Colonne stratigraphique simplifiée des secteurs du mont Bourbeau et du lac Berrigan .......... 21

TABLEAU 2: Chronologie des divers réseaux de failles, modèle de travail tentatif .............. 39

TABLEAU 3: Teneurs des minéralisations provenant des principaux indices décrits ............. 55

TABLEAU 4: Sommaire des différents indices minéralisés et de leurs caractéristiques ............... 88 


\section{LISTE DES PLANCHES}

page

PLANCHE I (A) Alternance de lits grèseux et argileux contenant des horizons graphiteux, riches en sulfures disséminés (pyrite) $\ldots \ldots \ldots \ldots \ldots \ldots \ldots \ldots$

I (B) Alternance de lits silteux et grèseux d'épaisseurs millimétriques à centimétriques Au sud du lac Devilliers, mont Bourbeau ....... 126

PLANCHE II (A) Sédiment mixte contenant des fragments mafiques porphyriques en plagioclase incorporés à une matrice composée de cristaux libres de plagioclase et de quartz. A $1^{\prime}$ est du lac Devilliers, Formation de Blondeau. Lumière

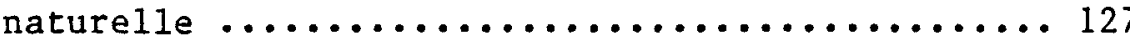

II (B) Coulée pyroclastique oligolithique composée de cristaux libres de plagioclase séricitisés, de quartz résorbés et de fragments d'opaques (pyrite). Au sud du lac Devilliers, mont

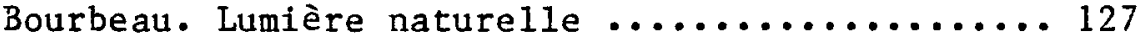

II (C) Sédiment mixte polygénétique contenant des fragments de volcanites mafiques de cristallinité variable (à noter la texture microlitique) et de volcanites felsiques, rhyolitiques (à fragments polycristallins de quartz). A 300 mètres au sud-est du lac Devilliers, Formation de Blondeau, mont Bourbeau (L.P.) $\ldots \ldots \ldots \ldots \ldots \ldots \ldots \ldots \ldots \ldots \ldots$

PLANCHE III (A) Lentille composée de fragments volcaniques monolithologiques de composition felsique, porphyriques et sub-anguleux. La tête du marteau indique 1 a polarité. Formation de Blondeau, indice du lac Devilliers, mont Bourbeau 128

III (B) Photomicrographie provenant d'un fragment rhyolitique de la lentille montrée précédemment. Ce fragment contient des phénocristaux altérés de plagioclase, d'amphibole aux bordures chloritisées et des cristaux de quartz bien résorbés. Formation de Blondeau, indice du lac Deviliers (L.N.) ..... 128 
III (C) Même microphotographie que précédemment. La matrice qui contient ces fragments est microcristalline et se compose majoritairement de

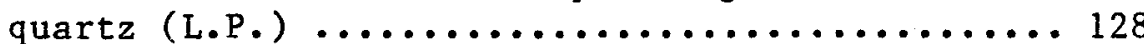

PLANCHE IV (A) Fragment pyriteux, montrant localement des textures colloformes. Ce fragment repose dans une coulée de débris oligolithologique. Formation de

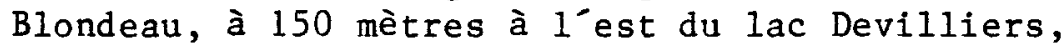
mont Bourbeau ......................... 129

IV (B) Bombe volcanique (?), de composition felsique et porphyrique. Cette bombe est recouverte successivement par une mince épaisseur de sédiments fins, grèseux à silteux, et par une coulée de débris. La tête du marteau indique la polarité. Indice du lac Devilliers, mont Bourbeau 129

PLANCHE V (A) Sédiments grèseux lités, tronqués par une coulée de débris composée de fragments volcaniques intermédiaires à felsiques. A 300

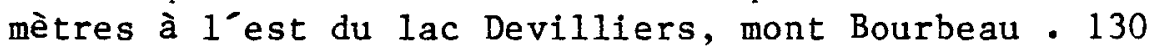

V (B) Base d'une coulée basaltique massive et variolaire. Formation de Blondeau, indice du lac Devilliers, mont Bourbeau ................ 130

PLANCHE VI (A) Intrusif (?) de composition pyroxénitique, sus-jacent aux laves variolaires. La tête du marteau repose sur le basalte. Formation de

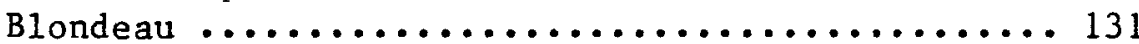

VI (B) Basalte intensément bréchifié et localement variolaire, en bordure de la trace axiale du synclinal de Chibougamau. Formation de Blondeau . 131

PLANCHE VII (A) Wherlite, composée de cristaux légèrement serpentinisés d'olivine (gris pâle) et de clinopyroxènes ouralitisés fréquemment poecilitiques (noirâtre). Membre inférieur du filon-couche de Roberge, au sud-ouest du lac

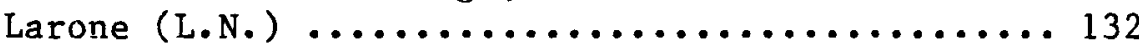

VII (B) Microphotographie montrant une alternance abrupte entre des bandes de composition dunitique (pâle), composées exclusivement de cristaux ovoĭdes d'olivine, et pyroxénitique (foncée). Membre inférieur du filon-couche de Roberge, au sud du mont Bourbeau ....................... 132 
PLANCHE VIII (A) Bande de composition gabbroĩque parallèle au litage magmatique observé à 1 'intérieur de la pyroxénite verte du filon-couche de Ventures. Cette pyroxénite est également bien foliée. Mont

Bourbeau ............................ 133

VIII (B) Dyke gabbroĩque injecté dans 1a pyroxénite verte du filon-couche de Ventures. Des minéralisations en pyrite empruntent les ordures et fractures de ce dyke et $s^{-1} y$ retrouvent aussi disséminées. Mont Bourbeau ............................133

PLANCHE IX (A) Pyroxénite gabbroíque foliée, provenant de la zone de transition entre la pyroxénite et le gabbro. Les clinopyroxènes sont ouralitisés. Filon-couche de Ventures, mont Bourbeau. (L.N.) . 134

IX (B) Contact basal entre la bordure de trempe du filon-couche de Bourbeau (sous la tête du marteau) et les sédiments grèseux et lités de la Formation de Blondeau. A 300 mètres à $1^{\prime}$ est du lac Devilliers, mont Bourbeau ............. 134

IX (C) Sédiment grèseux, composé de cristaux libres de plagioclase légèrement séricitisés et de quelques cristaux de quartz. Ce sédiment est plus ou moins silicifié et chloritisé. Formation de Blondeau, à la base du filon-couche de Bourbeau, mont

Bourbeau. (L.P.) ...................... 134

PLANCHE $X$ (A) Microphotographie du membre dunitique basal du filon-couche de Bourbeau, composé de cristaux ovoĭdes d'olivine (pâle) et de cristaux poecilitiques d'orthopyroxène (bronzite ?). A 400

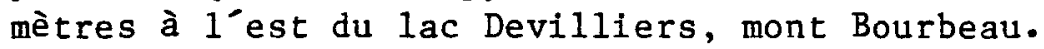

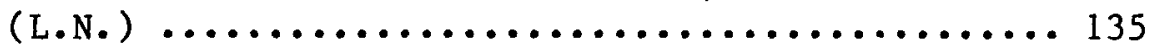

$X$ (B) Lentille "pegmatitique" de composition gabbroìque prenant place dans le ferrogabbro du filon-couche de Bourbeau. Au NW du lac Larone ... 135

PLANCHE XI (A) Microphotographie d'un dyke à phénocristaux de plagioclase peu altérés et de quartz. La matrice se compose pour la plus grande part de quartz microcristallin. Indice des Dômes du radar, mont

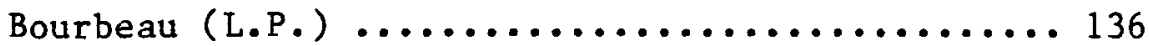

XI (B) Microphotographie $d^{-}$un dyke porphyrique en pyroxènes, totalement ouralitisés. La matrice consiste en un assemblage $d^{\prime}$ aiguilles $d^{-}$amphibole (actinote), de chlorite et de rares feldspaths. Formation de Haüy (?), à 1 'ouest du lac Ham. 


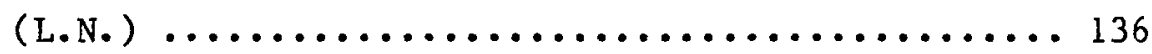

PLANCHE XII Leucotonalite massive (L) bordée dans sa partie supérieure par une syénite porphyrique en amphibole ( $S$ ). Une veine composée d'axinite (rosée) et localement d'épidote (vert) recoupe ces deux lithologies. Bloc rocheux provenant de la galerie d'exploration de 1'indice "Bel-3", mont

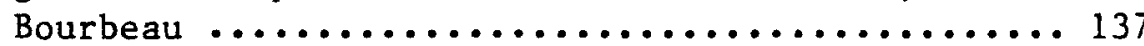

PLANCHE XIII (A) Leucotonalite. Cette roche se compose de phénocristaux de plagioclase, séricitisés de façon variable, d'amphibole et de quartz. Stock tonalitique du lac Line, échantillon recueilli au sud de la Baie du Cran Penché. (L.N.) ......... 138

XIII (B) Leucotonalite. L“altération des phénocristaux de plagioclase et d'amphibole est peu intense. La matrice consiste quasi exclusivement de cristaux de quartz microcristallin et de rares feldspaths

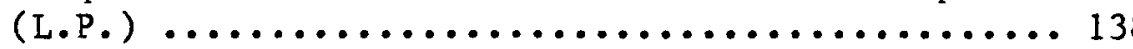

PLANCHE XIV (A) Microphotographie d'une syénite porphyrique en amphibole (foncé) et en feldspath (orthose) légèrement séricitisés. Echantillon provenant de la galerie d'exploration de 1'indice "Be1-3",

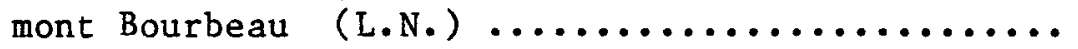

XIV (B) Faciès dioritique de bordure du stock du lac Line (gris noirâtre) en contact graduel avec une leucotonalite (sous le capuchon). Cette diorite est souvent foliée parallèlement au contact avec les volcanites encaissantes. Au nord-ouest du

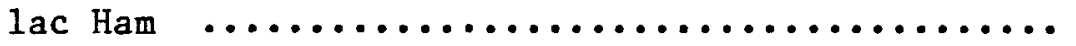

XIV (C) Faciès de cornéenne résultant du métamorphisme de contact amené par le stock tonalitique du lac Line et qui est observé dans des sédiments grèseux épiclastiques et lités de la Formation de Blondeau. Cet affleurement se trouve à moins de 100 mètres de 1 a bordure de ce stock et à environ 400 mètres au sud-est du lac Line. Mont Bourbeau ......... 139

PLANCHE XV Pli d'entrainement serré observé dans une péridotite serpentinisée, membre supérieure du filon-couche de Roberge. Le nord est du côté gauche. Ce pli, un anticlinal, possède une charnière inclinée vers $1^{\text {'est et }}$ il se situe en bordure nord d'une faille inverse majeure, $\mathrm{d}^{\prime}$ orientation $\mathrm{E}-\mathrm{W}$. Ces caractéristiques signifient que la composante horizontale du mouvement global le long de cette faille est 
dextre. Au nord des indices des Dômes du radar, mont Bourbeau .......................... 140

PLANCHE XVI (A) Microphotographie de fragments basaltiques silicifiés, contenant des microlites de plagioclase. La matrice est constituée de fragments plus petits de chlorite, de quartz et de carbonate. Formation de Gilman, indice du

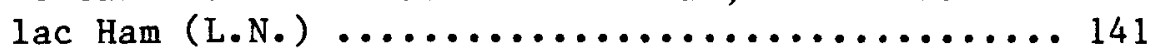

XVI (B) Filonets riches en pyrite et en chalcopyrite qui bréchifient une minéralisation antérieure composée de magnétite massive. Indice du lac Ham . 141

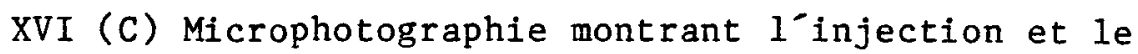
comblement par la chalcopyrite (jaune foncé) de fractures dans des grains de pyrite recristallisés sous forme de porphyroblastes (jaune pâle) et de magnétite. Indice du lac Ham. Lumière réfléchie ...........................

PLANCHE XVII (A) Lentilles pyriteuses stratiformes reposant sur un sédiment épiclastique. La tête du marteau donne la polarité. Formation de Blondeau, au sud

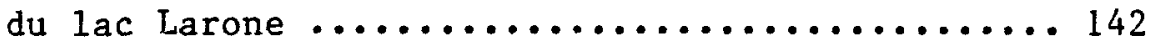

XVII (B) Détails de la photographie précédente. Les lits minéralisés sont très minces et ne contiennent que de la pyrite $. . \ldots \ldots \ldots \ldots \ldots \ldots \ldots \ldots \ldots . . . \ldots$

PLANCHE XVIII (A) Veinules discordantes minéralisées en pyrrhotine et plus ou moins en pyrite. Les sédiments épiclastiques encaissants sont fortement carbonatisés, chloritisés et silicifiés. La tête du marteau souligne la direction générale de la stratification et donne la polarité. Formation de Blondeau, indice du lac Devilliers ............ 143

XVIII (B) Fragment minéralisé plissé similaire aux minéralisations stratiformes stratigraphiquement sousjacentes. Ce fragment est incorporé à une coulée basaltique. Formation de Blondeau, indice

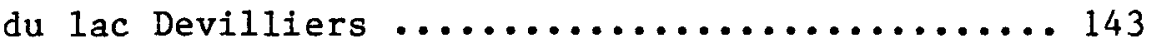

PLANCHE XIX (A) Début de carbonatisation, de silicification et de chloritisation autour de nucleus constitués de petits fragments et de cristaux. Formation de Blondeau, indice du lac Devilliers ............ 144

XIX (B) Intensification du processus d'altération observé sur la photographie précédente. Altérations préférentielles démontrées par la carbonatisation 
et la silicification, dessinant de pseudo-fragments blanchâtres sur 1'affleurement et se propageant de façon préférentielle selon la stratification. La chloritisation affecte davantage la matrice (gris verdâtre) et attaque également les zones préalablement carbonatisées et silicifiées, accentuant ainsi $1^{-}$aspect de pseudo-fragments observés sur cet affleurement .. 144

PLANCHE XX (A) Microphotographie d'un sédiment volcaniclastique grèseux montrant des pseudo-sphérulites produites par 1'altération hydrothermale. Le coeur $\mathrm{d}^{\prime}$ un sphérulite est intensément carbonatisé et séricitisé tandis que la bordure est plutôt chloritisée et silicifiée. Formation de Blondeau, indice du lac Devilliers (L.N.) ................. 145

$\mathrm{XX}$ (B) Même microphotographie que précédemment. La bordure du sphérulite est enrobée de quartz microcristallin. La matrice est intensément cloritisée. Ces caractéristiques témoignent du caractère précoce de la carbonatisation, de la continuité de la silicification et de I'apparition ultérieure de la chlorite (L.P.) ... 145

PLANCHE XXI (A) Minéralisations rubannées en chalcopyrite (jaune. foncé) et en pyrite idiomorphe (jaune pâle). Les paillettes de tourmaline (foncée) soulignent I'aspect rubanné. Formation de Blondeau, indice des Dômes du radar, mont Bourbeau. Lumière réfléchie .......................... 146

(B) Fenêtre au travers du conglomérat protérozoíque. Celle-ci permet de voir des filonets minéralisés recouverts en discordance par ces sédiments. Indice des Dômes du radar, mont Bourbeau ........................... 146

(C) Veine de molybdénite (indiquée par la flèche) tapissée de carbonate et encaissée dans un cisaillement d'orientation $\mathrm{N}-\mathrm{S}$. Les épontes sont intensément silicifiées. Indice des Dômes du radar, mont Bourbeau ................... 146

PLANCHE XXII (A) Vue en détail d un pseudo-fragment de couleur blanchâtre. Une veinule minéralisée en chalcopyrite, sphalérite et pyrite est bordée par une forte altération en chlorite. La séricitisation est observable à partir d'une vingtaine de centimètres de cette veinule. Une seconde fracture, cette fois stérile, borde du côté droit ce pseudo-fragment. Formation de

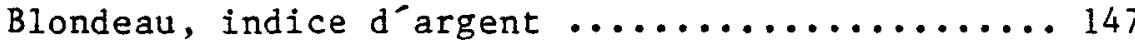


XXII (B) Ces veinules minéralisées se composent de sphalérite (gris pâle) contenant fréquemment de fines inclusions de chalcopyrite (jaune foncée), de pyrite ( $j$ aune blanchâtre) et de gangue (gris foncé). De la millérite (indiquée par la flèche) a possiblement été identifiée. Indice $d^{\prime}$ argent (L.R.) $\ldots \ldots \ldots \ldots \ldots \ldots \ldots \ldots \ldots \ldots \ldots$

PLANCHE XXIII (A) Patron de pseudo-bréchification dans les épontes des fractures, causé par la nature distincte des altérations en présence: la chloritisation et la séricitisation. Formation de Blondeau, indice $\mathrm{d}^{\top}$ argent, mont Bourbeau ................... 148

XXIII (B) Détails sur 1'intensité et la position respective des altérations. Le front de chloritisation (gris-bleuté) se restreint aux bordures immédiates des fractures, minéralisées ou pas, tandis que la séricitisation affecte les roches plus éloignées (blanchâtres). A noter $q u^{\prime} i l n^{\prime} y$ a pas de déplacement apparent des volcaniclastites de part et $d^{\prime}$ autre des fractures et des différents fronts d'altérations. La silicification est faible mais affecte une bonne partie de $1^{\prime}$ affleurement ...... 148

PLANCHE XXIV (A) Coulée pyroclastique de même composition que la roche de la planche XXIV (B) mais ici échantillonnée à $1^{\prime}$ intérieur du front de séricitisation. Les plagioclases ne laissent que deviner leur silhouette, tant la séricitisation est grande. La matrice est un peu plus chloritisée que dans $1^{\prime}$ exemple précédent (L.P.) . 149

XXIV (B) Coulée pyroclastique composée de cristaux libres de plagioclase faiblement séricitisés, d’amphibole et de quartz. La matrice est silicifiée et faiblement chloritisée. Cet échantillon se situe à environ 3 mètres des fractures minéralisées, à 1 'extérieur du front de séricitisation. Formation de Blondeau, indice d'argent (L.P.) ........... 149

PLANCHE XXV Faille d'orientation NNW, séparant les couches bien litées de la Formation de Blondeau d'avec la bordure de trempe basale du filon-couche de Bourbeau. Des veines minéralisées sont sub-parallèles à cette structure et se retrouvent préférentiellement du côté des

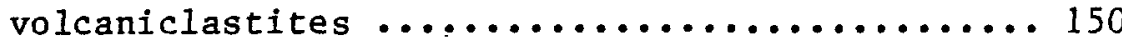

PLANCHE XXVI (A) Minéralisations en pyrrhotine, plus ou moins massive, contenant des inclusions de gangue. Dans 
la Formation de Blondeau, près du sommet du filon-couche de Ventures, mont Bourbeau (L.R.) . 151

XXVI (B) Même microphotographie que précédemment. La gangue se compose principalement de quartz microcristallin, de carbonate et de quelques minéraux mafiques totalement chloritisés (L.P.)

PLANCHE XXVII (A) Minéralisations composées de pentlandite (jaune clair), de chalcopyrite (jaune foncé), de pyrrhotine (gris pâle) et de sphalérite. Des opaques (phyllosilicates) soulignent un vague rubannement dans la partie droite de la microphotographie. Filon-couche de Ventures, indice "Be1-3", mont Bourbeau (L.R.) ..........

XXVII (B) Plissotements démontrés par des bandes riches en phyllosilicates bordées par la sphalérite (gris), la pyrrhotine (gris rosé) et la chalcopyrite (jaune foncé). Indice "Be1-3", mont Bourbeau (L.R.) .............................

XXVII (C) Texture "decussate" démontrée par la pyrrhotine, les bordures en points triples sont fréquentes. Indice "Be1-3", mont Bourbeau (L.R.) .......... 152

PLANCHE XXVIII (A) Cristaux aciculaires de molybdénite (jaune pâle) et de pyrite (blanc) enserrés dans une gangue constituée principalement de quartz et de carbonate (jaune grisâtre). Stock tonalitique du lac Line, au sud de la Baie du Cran Penché (L.R.) $\ldots \ldots \ldots \ldots \ldots \ldots \ldots \ldots \ldots \ldots \ldots \ldots \ldots \ldots \ldots$

PLANCHE XXVIII (B) Brèche dans la leucotonalite, cimentée par un carbonate de fer brunâtre. Présence localement de traces de pyrite. Les fragments tonalitiques sont sub-anguleux et ne semblent pas s'être beaucoup déplacés. Stock tonalitique du lac Line, au nord-ouest du lac Ham 


\section{CHAPITRE 1}

\section{INTRODUCTION}

\subsection{Situation géographique}

Les travaux de recherche, qui servent de base à ce mémoire, ont été faits sur un territoire de $18 \mathrm{~km} 2$, situé immédiatement au nord de la ville de Chibougamau dans le canton de McKenzie (figure 1). Ce terrain est délimité par les longitudes $74^{\circ} 18^{\circ} 30^{\prime \prime}$ et $74^{\circ} 24^{\circ} 00^{\prime \prime}$ et les latitudes $49^{\circ} 55^{\circ} 50^{\prime \prime}$ et $49^{\circ} 57^{\circ} 30^{\prime \prime}$. Il fait partie du feuillet 32 G/16 du découpage SNRC à $1: 50000$.

Les routes de Chibougamau en direction de la station-radar du mont Bourbeau de même que celles vers le club de golf et le lac Larone assurent un excellent accès à toutes les parties de ce terrain. Le mont Bourbeau représente le plus haut relief de ce secteur, avec une dénivellation de plus de 150 mètres.

\subsection{Buts du travail}

L'intérêt de ce teritoire réside dans le fait que diverses minéralisations en $\mathrm{Cu}, \mathrm{Zn}, \mathrm{Pb}, \mathrm{Mo}, \mathrm{Ag}$ et $\mathrm{Au} \mathrm{s}^{\prime} \mathrm{y}$ retrouvent. Celles-ci montrent des compositions, fréquences, distributions, altérations et

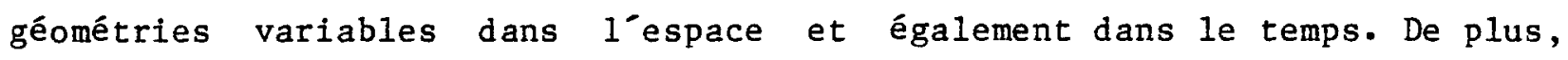
d’anciens producteurs aurifères, tels les mines Norbeau et Gwillim (Bouchard et al. 1984 et Bouchard, 1986), de cuivre, tel la mine Bruno (Trudeau, 1981), et un indice de zinc, or et argent, le prospect Taché (Pilote, 1984 et 1986), se situent à peu de distance dans les prolongements 


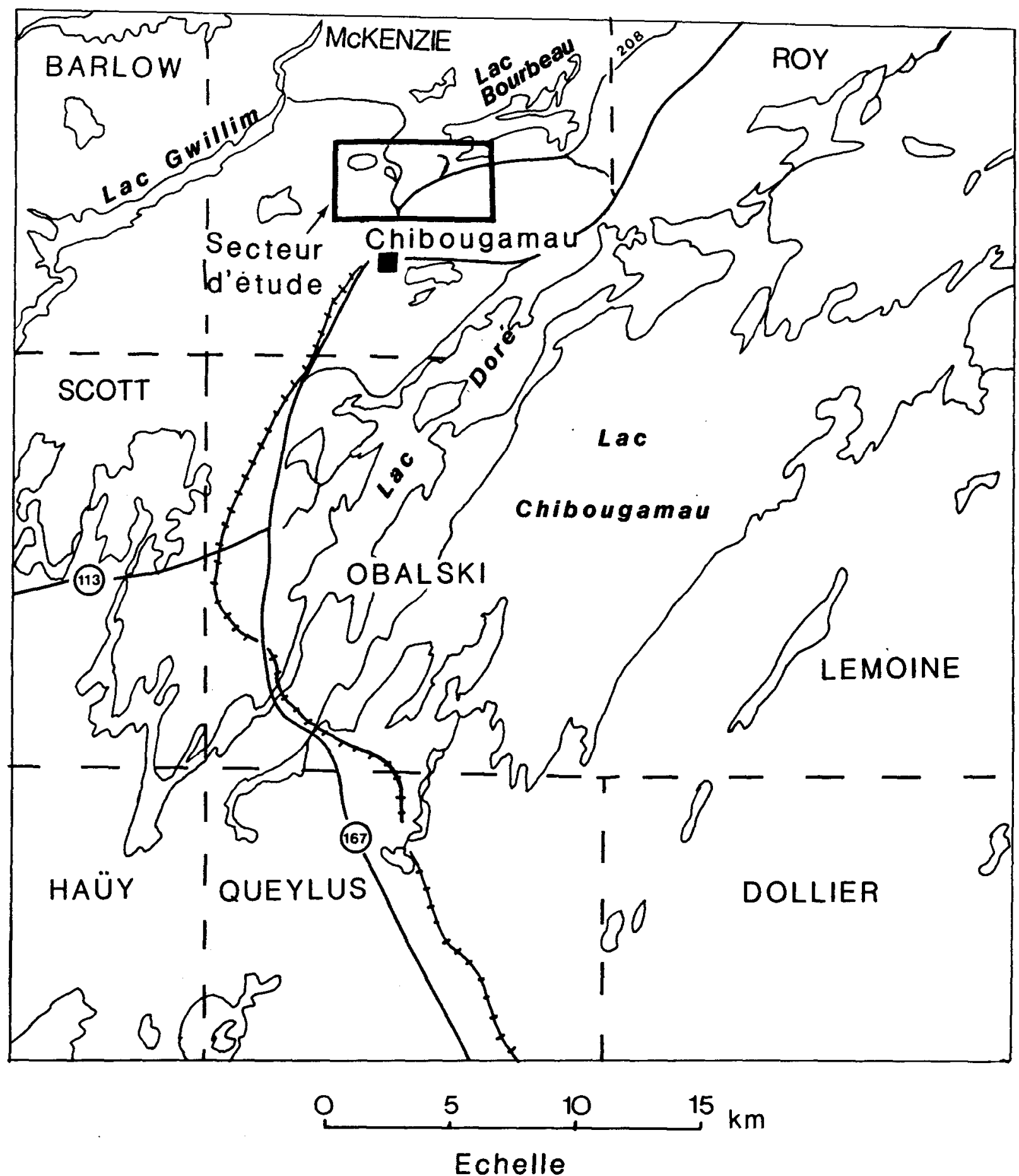

Figure 1 Localisation du secteur d'étude et géographie de la rëgion de Chibougamau 
est et ouest des lithologies rencontrées.

Les buts de ce travail se subdivisent ainsi en quatre volets:

(1) réviser et établir les bases de la stratigraphie locale d'après les modèles régionaux;

(2) répertorier et classifier tous les sites minéralisés rencontrés;

(3) localiser et décrire ces sites à l'intérieur du contexte stratigraphique local;

(4) discuter des différents contrôles exercés sur ces minéralisations et proposer une ou des origines possibles pour les différents types de minéralisations rencontrées.

\subsection{Travaux antérieurs}

Dès 1870 , James Richardson visitait la région pour le compte de la Commission Géologique du Canada. Par la suite, différents prospecteurs et compagnies minières se sont intéressés, quelques-uns avec succès, à la région.

Le canton de McKenzie a d’abord fait $1^{\prime}$ 'objet d'une première campagne de cartographie systématique par Retty (1929) à 1'échelle 1:63 360. En 1935 parait le rapport de Mawdsley et Norman. Ce travail remarquable, à 1'échelle 1:63 360, a jeté les bases de tous les travaux géologiques dans le secteur de Chibougamau. En 1950, le Gouvernement du Québec entreprend la cartographie détaillée du camp minier de Chibougamau. En 1960, le rapport de Smith et Allard est édité. Smith a couvert alors le quart $\mathrm{SW}$ et la demie nord du quart SE du canton de McKenzie à 1'éche1le 1:12 000. De 1962 à 1966, Duquette $(1976,1982)$ cartographie la moitié nord des cantons de 
McKenzie, Roy et le quart nord-ouest du canton de McKorki11. Il définit la structure synforme régionale représentée par le synclinal de Chibougamau. Il est de plus $1^{\prime}$ auteur de la première synthèse géologique sur la région en 1970. Allard et a1. (1979) ont reconsidéré ces travaux lors d'une seconde synthèse géologique.

Du coté des compagnies minières, un grand nombre ont oeuvré sur ce terrain. Mentionnons entre autres, des années 40 à nos jours: Sodem; Belle Chibougamau Mines Ltd; Hailebury Mining Syndicate; International Mining Corp.; Royran Goldfield; Quebec Yellowknife Gold Mines Ltd; New Royran Copper Mines Ltd; Consolidated Quebec Yellowknife Mines Ltd; Marvelor Mines Ltd; Newlund Mines Ltd; Dupont Mining Co. Ltd; Taché Lake Mines Ltd; Chibougamau Kayrand Copper Mines Ltd; Ran Mar Mines Ltd; Amalgamated Beau-Belle Mines Ltd; Bruno Mining Corp.; Dundee-Palliser Resources Inc.; Brosnan Chibougamau Mines Ltd; Normandy Chibougamau Mines Ltd; Soquem; Mattagami Lake Mines Ltd et Campbell Chibougamau Mines Ltd (les Mines (amchib Inc.).

\subsection{Méthodes de travail}

Diverses parties de ce territoire ont été cartographiées au cours des étés 1982 et 1983 pour le compte du Ministère de 1'Energie et des Ressources du Québec. Une carte préliminaire au 1:10 000 a été publiée en 1983 (Pilote, 1983). Nous avons fait des cheminements systématiques, espacés de $150 \mathrm{~m}$ au maximum en terrain non-jalonné ou encore utilisé les grilles coupées par différentes compagnies minières. Les rapports géologiques tant de ces compagnies que des gouvernements ont servi à 1 a 
compilation des informations.

Sur ce territoire, nous avons recueilli plus de 450 échantillons au cours de ces deux étés. De ce nombre, 120 lames minces et 30 sections polies ont été tirées et étudiées au cours des hivers subséquents (de 1983 à 1985). Quelques 50 analyses totales et une quarantaine pour les substances d'intérêt économique. ont été faites sur des échantillons sélectionnés. Le Ministère de 1'Energie et des Ressources du Québec (Centre de Recherches Minérales) a procédé à 1'exécution des différentes analyses chimiques.

Les travaux de laboratoire ont été accomplis dans les locaux du Département des Sciences Appliquées de 1'Université du Québec à Chicoutimi.

\subsection{Remerciements}

Je remercie sincèrement les membres de mon comité de thèse, MM Jayanta Guha (directeur), Jacques Carignan et Erich Dimroth pour 1'aide, les nombreux conseils et commentaires pertinents apportés lors de 1'élaboration de cette thèse.

Les travaux sur le terrain furent défrayés par le Ministère de 1'Energie et des Ressources du Québec au cours des étés 1982 et 1983. Un soutien financier fut également accordé par le fonds F.C.A.C. (action concertée). J'exprime également toute ma gratitude envers André Gobeil, Gilles 0. Allard et Réal Daigneault, géologues du M.E.R., pour les diverses excursions et discussions auxquelles nous avons participé à Chibougamau et 
qui nous furent particulièrement profitables.

Je remercie aussi les Mines Camchib Inc. ainsi que leur personnel (André Tremblay et Marie Racine) pour leur excellente coopération et échange de point de vue. Je veux remercier finalement tout le personnel du Module des Sciences de la Terre, particulièrement Yvon Boudreault et J.-E. Villeneuve, qui nous ont aidé en différents moments dans nos travaux. Claude Dallaire, Claire Tremblay et Jules Tremblay ont apporté leur collaboration au dessin des différentes figures et cartes. 
CHAPITRE 2

GEOLOGIE REGIONALE

\subsection{Introduction}

Le camp minier de Chibougamau fait partie de la province Supérieure du Bouclier Précambrien et de la ceinture orogénique archéenne de 1'Abitibi (Goodwin et Riddler, 1970). Cette ceinture est marquée par une évolution asymétrique correspondant à deux subdivisions majeures: la zone interne et une zone externe (Dimroth et al., 1982). Les roches de 1a région de Chibougamau s'insèrent à 1'extrémité est du sillon de roches vertes Chibougamau-Matagami qui prend place dans une zone interne (figure 2). Cette dernière se distingue entre autres de la zone externe par la présence d'importants complexes ignés lités et de plutons tonalitiques-trondhjémitiques syn-volcaniques et par $1^{` a}$ absence à 1'échelle régionale de komatiite à la base de l'empilement volcanique (Dimroth et al., 1982 et $1984 b$ ).

Le sillon Chibougamau-Matagami, orienté est-ouest, s'étend sur une distance d'environ $440 \mathrm{~km}$ avec une largeur variant de 25 à $100 \mathrm{~km}$. Il est bordé à 1'ouest par la sous-province de Kapuskasing. Du coté est, ce sillon se poursuit sur une distance non-déterminée dans la Province de Grenville (Allard, 1978 et 1979). Au nord, il est limité par des terrains gneissiques et des massifs granitoìdes archéens d’âges tectoniques divers (Racicot et a1., 1984). 


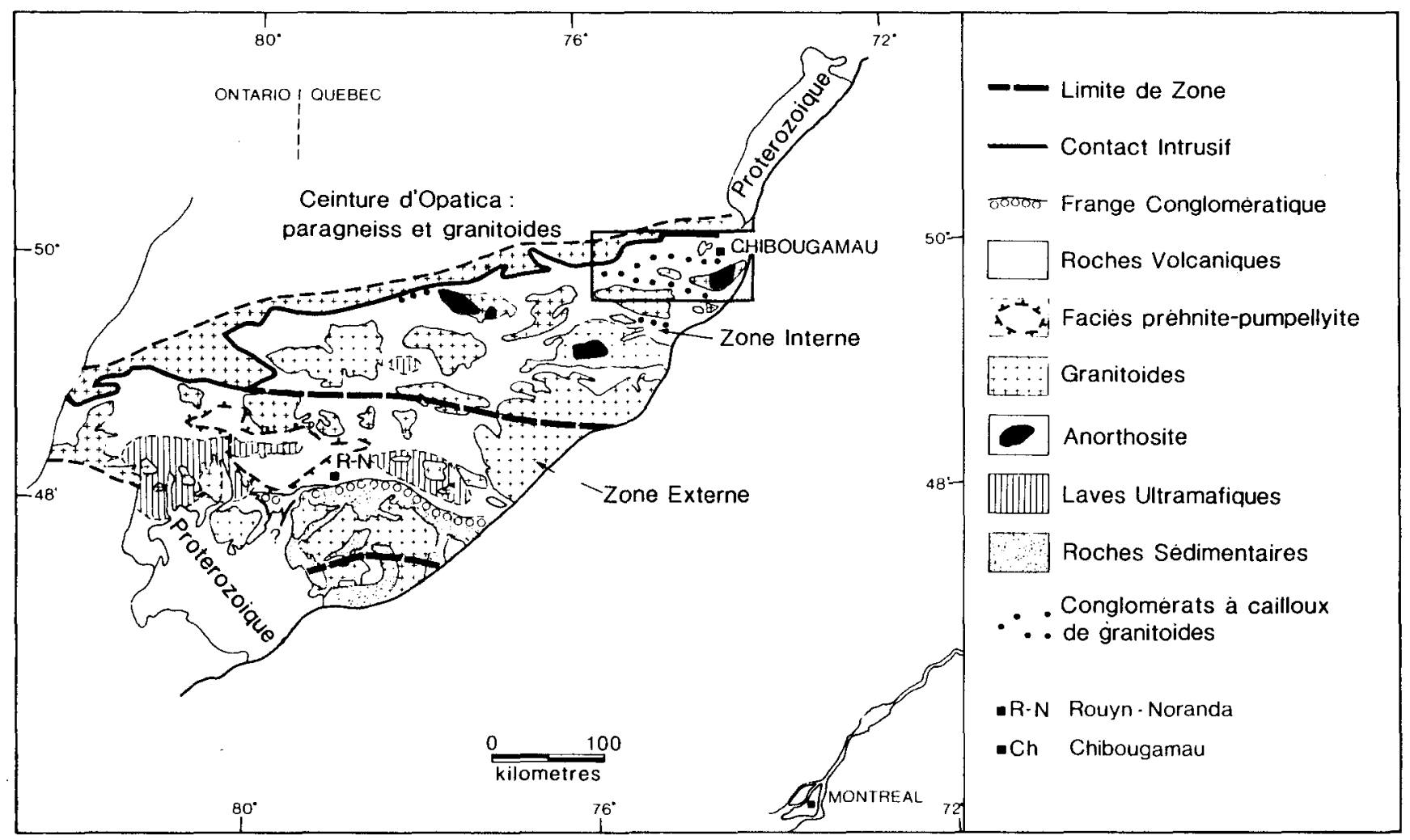

Figure 2. Localisation de la région de Chibougamau et subdivision de la ceinture orogénique de 1 -Abitibi en des zones interne et externe. Modifié de Dimroth et a1. (1982). 


\subsection{Généralités}

Les unités lithologiques archéennes de la région (figure 3) ont été divisées en 2 groupes (Allard et a1., 1979): à la base, le Groupe de Roy (Duquette, 1970) et au sommet le Groupe d'Opémisca, reposant sur le premier en discordance plus ou moins marquée, selon les localités (figure 4).

Le Groupe de Roy se compose, dans la région de Chibougamau, de deux cycles volcaniques distincts, chacun allant $d^{\top}$ une composition mafique à felsique (Allard et al., 1979). Les Formations d'Obatogamau et de Waconichi représentent le premier cycle tandis que le second est constitué par les Formations de Gilman et de Blondeau. La Formation de Bordeleau (Caty, 1979), exposée uniquement dans le synclinorium de Waconichi, est rattachée à ce groupe.

Le Groupe d'Opémisca contient de façon conventionnelle les Formations de Daubrée, Stella, Chebistuan et Hauy.

Des lambeaux de roches protérozoĩques d’origine glaciaire (Barlow et a1., 1911; Retty, 1929; Mawdsley et Norman, 1935; Long, 1974) sont identifiés à la Formation de Chibougamau. Celle-ci est composée de conglomérats, arkoses, grès et tillites qui reposent en discordance sur les roches archéennes.

Des dykes tardifs de diabase recoupent toutes les lithologies présentes dans la région. Ceux-ci sont présumés appartenir au réseau de dykes de 1'Abitibi (Fahrig et a1., 1965). 


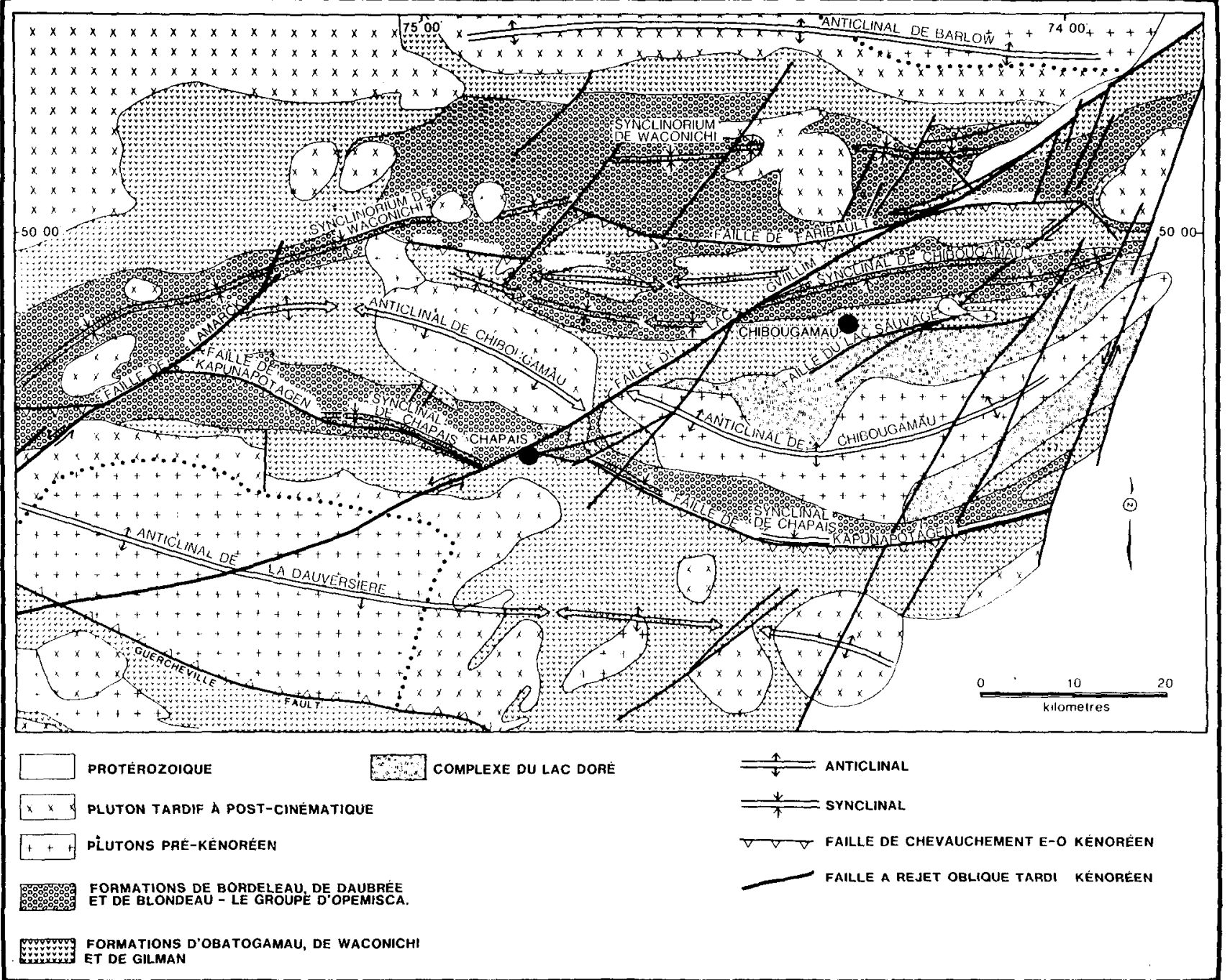

Figure 3. Carte tectonique simplifiée de la région de Chibougamau. Ti rée de Dimroth et al. (1985a)et modifiée de Gobeil et Racicot (1983). 


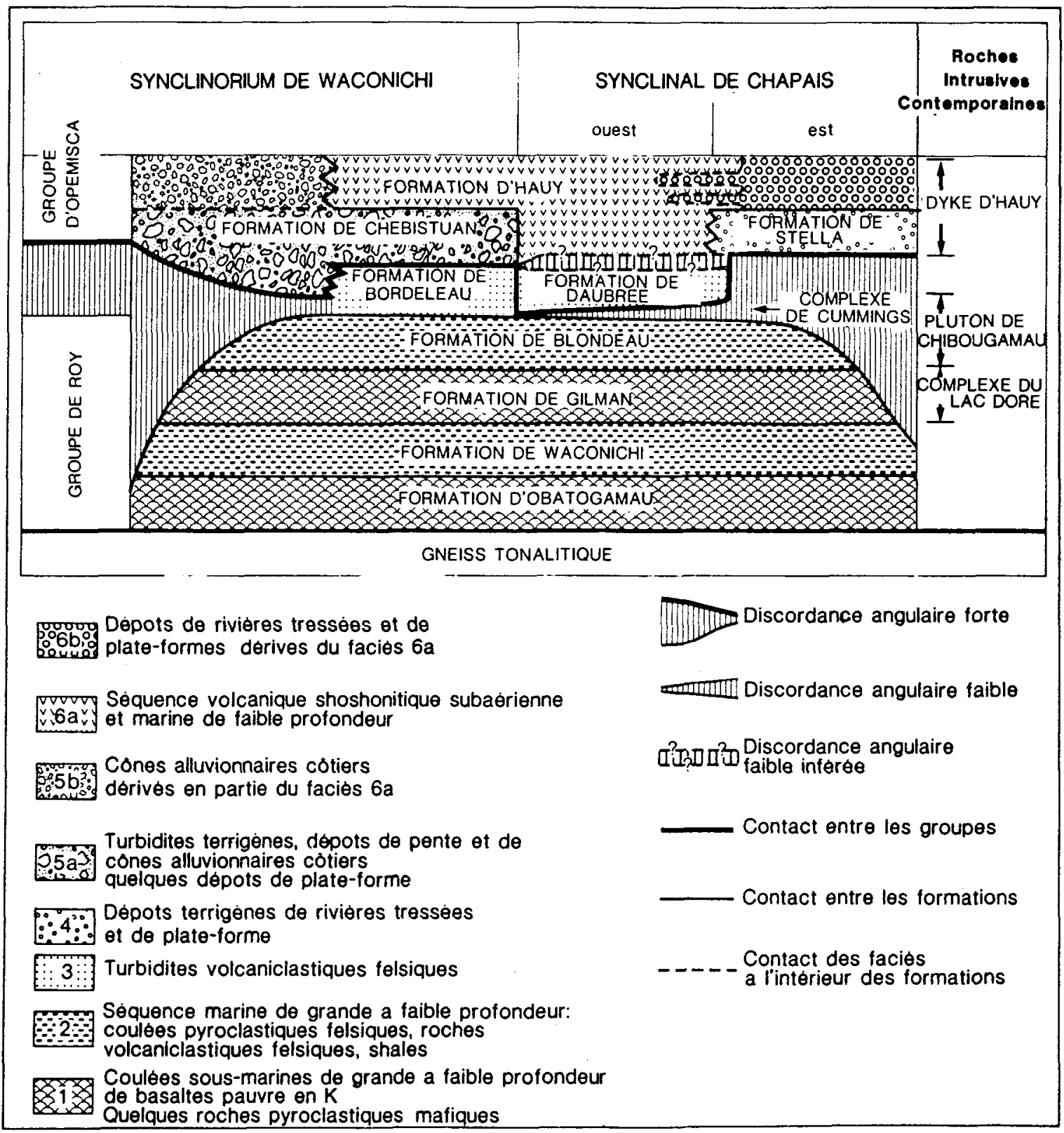

Figure 4. Représentation schématique de la stratigraphie archéenne dans les régions de Chibougamau et de Chapais. Modifiée de Dimroth et al. (1985a). 


\subsection{Groupe de Roy}

La Formation d'Obatogamau (Cimon, 1977) se compose surtout de laves mafiques massives à coussinées et de filons-couches comagmatiques. Nous y retrouvons aussi de minces épaisseurs de sédiments, telles des argilites graphiteuses et des exhalites. Une caractéristique de ces laves mafiques et filons-couches est qu'ils sont par endroit porphyriques en plagioclase, ceux-ci pouvant constituer localement plus de $10 \%$ de $1 a$ roche. Cette formation a une épaisseur estimée de 3.5 à $5 \mathrm{~km}$ et une extension régionale est-ouest d’au-delà de $100 \mathrm{~km}$. A l'extérieur de la région de Chibougamau, cette formation comprend également des petits centres volcaniques felsiques ponctuels, tel celui du lac des Vents (Gobeil et Racicot, 1983; Tait et al., 1985).

La Formation de Waconichi (Duquette, 1970) représente une interruption plus ou moins bien développée et discontinue entre 1'épanchement des volcanites mafiques des Formations d'Obatogamau et de Gilman. Elle est d'épaisseur restreinte, de 200 à 500 mètres et marquée par la présence d’amas lenticulaires de caractère volcanogène felsique. La Formation de Waconichi comprend des rhyolites et dykes porphyriques, des pyroclastites felsiques et des coulées volcaniques mafiques. Des formations de fer marquent localement le sommet de ces roches (Henry et Allard, 1979).

La Formation de Gilman a une épaisseur de 2 à $5 \mathrm{~km}$. Elle est principalement constituée de laves mafiques massives à coussinées, de filons-couches gabbroiques comagmatiques et d'horizons pyroclastiques. 
L'épaisseur de cette formation est à son maximum dans les environs de la ville de Chibougamau. Ceci suggère un complexe central volcanique à cet endroit (Allard, 1984). La partie supérieure de cette formation est présente sur le terrain cartographié.

La Formation de Blondeau (Duquette, 1970) représente un assemblage volcano-sédimentaire comprenant, selon les faciès distaux (Archer, 1983) ou proximaux, diverses proportions de sédiments remaniés, des coulées pyroclastiques et rhyolitiques, des tufs felsiques, des exhalites, des schistes bitumineux pyritifères et des coulées mafiques variolitiques. L'épaisseur de cette formation est d'environ un kilomètre. Son chimisme est représentatif d’une suite sub-alcaline à caractère tholéiitique pauvre en potassium (Trudel et Cloutier, 1984).

La Formation de Bordeleau a été identifiée par Caty (1979) comme reposant de façon conforme sur la Formation de Blondeau, dans le Synclinorium de Waconichi. Cette formation a plus de $1 \mathrm{~km}$ d’épaisseur. Elle est composée quasi essentiellement de turbidites volcaniclastiques felsiques (Dimroth et al., 1985a) pauvres en quartz.

2.4 Groupe d'Opémisca

Les Formations de Daubrée (Wolhuter, 1962; Dimroth, 1985a) et de Stella (Cimon, 1976) se situent à la base du Groupe d'Opémisca, respectivemnt dans les parties ouest et est du synclinal de Chapais. Globalement, la composition de la Formation de Daubrée est similaire à celle de la Formation de Bordeleau. Son épaisseur est d'environ $2 \mathrm{~km}$. 
La Formation de Stella représente une séquence de roches sédimentaires terrigènes fluviatiles et littorales (lentilles de conglomérats, grès, argilites) avec localement quelques coulées andésitiques. Cette formation, d'une épaisseur de 1 à $2 \mathrm{~km}$, marque la bordure d'un bassin de faille principalement continental (Dimroth et al., 1985a).

La Formation, de Chebistuan (Caty, 1977) a une épaisseur maximum de $4 \mathrm{~km}$. Elle se retrouve dans le synclinorium de Waconichi. Cette formation consiste en un assemblage de sédiments polymictes épiclastiques déposés dans un environnement de bassin de faille marin (Mueller et Dimroth, 1984).

La Formation de Haüy (Cimon, 1976) se compose, de façon caractéristique, de laves potassiques, intermédiaires à mafiques, porphyriques en pyroxène et plagioclase, émises de façon sub-aérienne. Son épaisseur varie de 1 à 2 km (Piché, 1984; Allard et al., 1979). Ces coulées de laves sont interlitées avec des tufs, grès, argilites et conglomérats. De par leur composition, ces sédiments proviennent en grande partie de 1'érosion de ces mêmes coulées de laves. La Formation de Haüy s'interdigite avec les Formations de Chebistuan et de Stella dans le synclinorium de Waconichi et le synclinal de Chapais (Dimroth et al., 1985a; Piché, 1984).

Des études sédimentologiques et volcanologiques entreprises 
récemment par Dimroth et al. (1984b, 1985a) ont permis d’établir de façon préliminaire le modèle palégéographique suivant pour la région de Chibougamau: 1) les Formations d'Obatogamau et de Gilman représentent 1'établissement et la croissance d'une chaîne volcanique sous-marine basaltique; 2) les Formations de Blondeau, Bordeleau et Daubrée indiquent 1’émergence et $1 a$ croissance d’îles volcaniques, de caractère plus

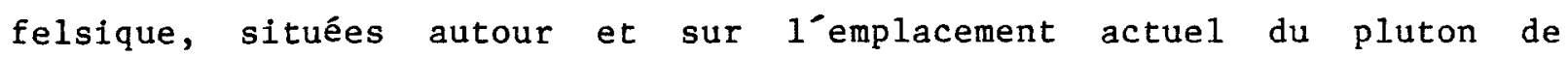
Chibougamau; 3) les Formations de Stella, Chebistuan et Hauy sont les

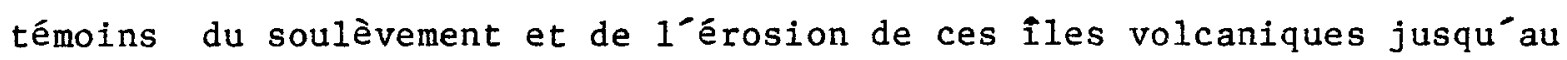
niveau des masses plutoniques subvolcaniques et d'une activité volcanique shoshonitique sub-aériennne. Les sédiments provenant de cette troisième phase se sont déposés dans des bassins de faille continentaux et marins, le synclinal de Chapais et le synclinorium de Waconichi.

\subsection{Roches intrusives}

Outre les filons-couches comagmatiques gabbroíques propres aux Formations d’obatogamau et de Gilman et quelques dykes porphyriques se retrouvant à des niveaux stratigraphiques bien précis, les roches du Groupe de Roy, dans la région de Chibougamau, sont envahies par d’autres masses synvolcaniques. Celles-ci sont par ordre stratigraphique ascendant le Complexe du lac Doré, le pluton de Chibougamau, le Complexe de Cummings et les dykes nourriciers rattachés aux coulées volcaniques de la Formation de Haïy.

Le Complexe du lac Doré (Allard, 1956 et 1976), de caractère anorthositique, est une large intrusion stratiforme de type Bushveld. Il 
est composé de 5 zones distinctes (Allard, 1984) qui sont, de la base au sommet: la zone anorthositique, la zone litée, la zone de ferrodiorite, la zone de sodagranophyre et la zone de bordure supérieure. L'épaisseur du complexe varie selon les localités de 4 à $7 \mathrm{~km}$. Du côté régional, il s'est globalement mis en place entre les Formations de Waconichi et de Gi1man.

La base du Complexe du lac Doré est brêchifiée par le pluton de Chibougamau, un dôme structural émergeant (Cimon et Gobeil, 1976)

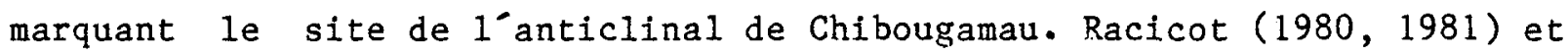
Racicot et al. (1984) ont reconnu quatre lithologies majeures dans ce pluton polyphasé, soit de la plus jeune à la plus vieille: la méladiorite à hornblende, la diorite à hornblende et quartz, la tonalite à biotite et la leucotonalite (trondjhémite). Des lithologies de compositions intermédiaires existent cependant.

Le Complexe de Cummings (Duquette, 1972 et 1976) se compose de trois filons-couches différenciés, comagmatiques (Poitras, 1984; Duquette, 1976), qui sont d'ultramafiques à mafiques en composition. Ce sont, de la base au sommet, les filons-couches de Roberge, de Ventures et de Bourbeau. Cet ordre est invariablement respecté dans la région de Chibougamau mais des répétitions et/ou des omissions, par failles ou par plis, sont parfois observées (Allard, 1976; Pilote et al., 1984a; Poitras, 1984; Daigneault et Allard, 1984). Ce complexe s'est mis en place dans la partie supérieure de la Formation de Gilman et à la base de 1a Formation de Blondeau. Sa très grande extension régionale, $160 \mathrm{~km}$ 
en direction est-ouest (Duquette, 1976), en fait un excellent horizon-repère.

Le filon-couche de Roberge est constitué d'un membre basal très bien lité de wehrlite, dunite et pyroxénite surmonté d’un membre supérieur dunitique plus massif (Poitras, 1984). L'épaisseur de ce filon-couche varie de 450 à $550 \mathrm{~m}$ au maximum. Il est 1'hôte de 1 'indice minéralisé ( $\mathrm{Zn}, \mathrm{Pb}, \mathrm{As}, \mathrm{Ag}$, $\mathrm{Au}$ ) Taché (Duquette, 1976; Pilote, 1984 et 1986).

Le filon-couche de Ventures repose directement sur le filon-couche de Roberge ou encore $i l$ en est séparé par une mince bande de roches volcaniclastiques appartenant à la Formation de Blondeau. Il est composé d'un membre pyroxénitique verdâtre surmonté d’un membre gabbroĩque. Son épaisseur totale varie de 150 mètres dans la section du lac Barlow (Poitras, 1984) à apparemment plus de 1100 mètres dans le secteur de Chapais (McMillan, 1972; Duquette, 1976 et 1982).

Le filon-couche de Bourbeau est séparé du filon-couche de Ventures par une épaisseur variable de roches de la Formation de Blondeau. I1 comprend une pyroxénite basale, un leucogabbro massif et un ferrogabbro-ferrodiorite à quartz. L'épaisseur du filon-couche de Bourbeau varie de 450 à 700 mètres. Il encaisse de plus $1^{\prime}$ ancienne mine

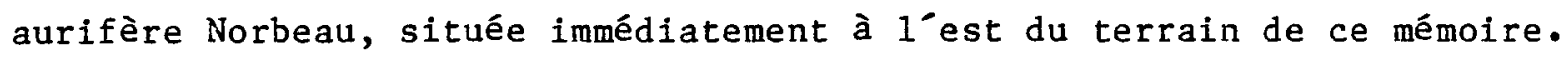

Finalement, des dykes porphyriques en pyroxène et plagioclase, 
caractéristiques de la Formation de Haüy, recoupent les roches de la partie supérieure du Groupe de Roy et le Groupe d’Opémisca. Ces dykes sont considérés comme les conduits nourriciers des laves de cette formation.

\subsection{Structure et métamorphisme}

Les roches supracrustales archéennes de la région de Chibougamau ont été plissées et métamorphisées au faciès des schistes verts (Duquette, 1970) lors de 1'orogénèse Kénoréenne, il y a 2.65 à 2.70 M.A. (Dallmeyer et al., 1975). Le patron structural résultant est dominé par la présence de larges synclinaux isoclinaux et de crètes anticlinales majeures, tous orientés est-ouest. Le synclinorium de Waconichi et les synclinaux de Chibougamau et de Chapais bordent, du nord au sud, les anticlinaux de Waconichi, de Chibougamau et de La Dauversière (figure 3 ). Le pendage des couches est fréquemment subvertical. Des plis précoces ouverts, de direction générale nord-sud, interfèrent localement avec les plis est-ouest pour donner des structures en dômes et bassins (Duquette, 1970; Allard et al. 1979; Daigneault et Allard, 1984).

Un deuxième événement dynamo-métamorphique, encore mal identifié, signalé autour de 2.2-2.0 M.A., semble avoir affecté toutes les lithologies alors présentes (Thorpe et al., 1981 et 1984). Cet événement parait correspondre entre autres aux minéralisations du secteur Henderson-Portage (Guha et al., 1983 et Guha, 1984) et à la ceinture de cisaillement observée au détroit de McKenzie dans le canton de Roy (Archambault et al., en préparation). Cette ceinture affecte également 
les sédiments protérozoĩques de la Formation de Chibougamau.

\begin{abstract}
Plusieurs réseaux de failles affectent la région minière de Chibougamau (Cimon, dans Allard et al., 1979). Quelques-unes de ces failles se signalent plus particulièrement puisqu'elles renferment ou bordent une grande partie des dépôts économiques connus jusqu’à maintenant. Globalement, les failles observées dans cette région sont orientées N-S, E-W, N-W, NE et NNE. Dans la région de Chibougamau plusieurs de ces directions sont ponctuellement associées aux ceintures de cisaillement du lac Gwillim (Dimroth et al., 1984a) et du lac Doré (Archambault et al., 1984 et 1986), selon les hypothèses de travail préliminaires de différents chercheurs. Ces ceintures sont d’âge syn à tardi-kénoréen alors que la ceinture de cisaillement du détroit de McKenzie est nettement post-kénoréenne (Dimroth et a1., 1984a; Guha et al., 1983; Dimroth et al., 1985b). Il est ainsi plus que probable que certaines failles ont pu être actives plus d'une fois dans $1^{\text {'histoire }}$ tectonique de la région.
\end{abstract}

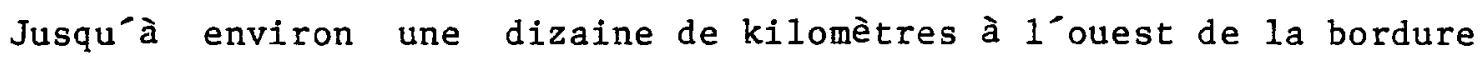
du front Grenville, $1^{\prime}$ orogénie Grenville est responsable de grands cisaillements orientés N-S à NNE $\left(020^{\circ}\right)$, affectant les roches de la Province Supérieure (Allard, 1979 et 1981; Daigneault et Allard, 1984). A proximité de ce front, de même qu'en bordure de certaines masses plutoniques importantes, le faciès métamorphique peut atteindre le degré amphibolite. 
CHAPITRE 3

GEOLOGIE LOCALE

\subsection{Introduction}

Le terrain étudié pour ce mémoire a été cartographié par Retty (1929) et par Mawdsley et Norman (1935) à 1'échelle 1:63 360. Smith a couvert la moitié sud du canton de McKenzie (1:12 000) et Duquette (1976, 1982) la moitié nord (1:12 000 et $1: 20000)$. Ces rapports ont permis d’établir les bases stratigraphiques et structurales de la présente étude. A 1'échelle régionale, le terrain étudié se situe sur le flanc sud du synclinal de Chibougamau, orienté grossièrement ENE et chevauche sa trace axiale au NW (carte en pochette). Les polarités sont régulièrement orientées vers le nord.

Les lithologies volcano-sédimentaires rencontrées ici sont d’âge archéen. Elles appartiennent pour la plupart à la partie supérieure du Groupe de Roy. Par ordre statigraphique (tableau 1), il sªgit des Formations de Gilman, de Blondeau et des trois filons-couches différenciés du Complexe de Cummings.

\subsection{Groupe de Roy}

3.2.1 Formation de Gilman

Cette formation présente sur ce terrain sa partie supérieure. Elle est composée de laves basaltiques massives à coussinées, de 


\begin{tabular}{|c|c|c|c|}
\hline ÂGE & FORMATION & & \\
\hline Cénozoïque & Sable et gravier & $10 \%$ & \\
\hline \multicolumn{2}{|c|}{ Discordance } & & \\
\hline Protérozoique & $\begin{array}{l}\text { FORMATION DE } \\
\text { CHIBOUGAMAU } \\
\text { Conglomérat } \\
\text { arkose } \\
\text { grès }\end{array}$ & & \\
\hline \multicolumn{2}{|c|}{ Discordance } & & \\
\hline \multirow[t]{2}{*}{ Archéen } & $\begin{array}{l}\text { FORMATION } \\
\text { DE BLONDEAU } \\
\text { Basaltes, andésites et } \\
\text { votcaniclastites } \\
\text { Sédiment volcaniclastique } \\
\text { shale,dykes porphyriques } \\
\text { en fetdspath et quartz, } \\
\text { basaltes variolitiques, turs }\end{array}$ & 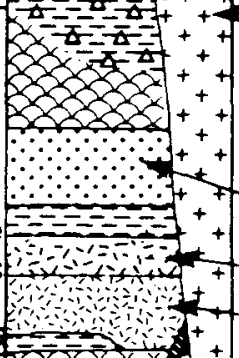 & $\begin{array}{l}\text { Stock tonalitique du } \\
\text { lac Line } \\
\text { (leucotonalite, syénite, } \\
\text { diorite) } \\
\text { Complexe de Cummings } \\
\text { filon-couche de Bourbeau } \\
\text { filon-couche de Ventures } \\
\text { filon-couche de Roberge }\end{array}$ \\
\hline & $\begin{array}{l}\text { FORMATION DE } \\
\text { GILMAN } \\
\text { Basalte, andésite, tufs, } \\
\text { sédiments } \\
\text { volcaniclastiques } \\
\text { et } \\
\text { filons-couchergabbröques }\end{array}$ & 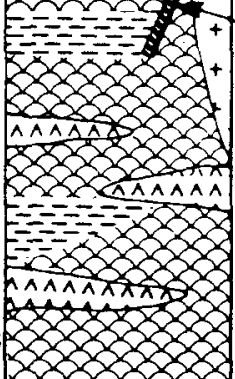 & $\begin{array}{l}\text { FORMATION DE HAÜY } \\
\text { (dyke à phénocristaux } \\
\text { pyroxénitique) }\end{array}$ \\
\hline
\end{tabular}

Tableau 1 Colonne stratigraphique simplifiée de la région du mont Bourbeau, canton de Mckenzie. Modifiée de Pilote et al. (1984a).

Représentation non à 1'échelle. 
filons-couches gabbroíques comagmatiques et de quelques rares lits de matériel volcaniclastique. La texture microlitique est générallement bien conservée dans ces laves. De plus, certaines coulées basaltiques sont localement variolaires. La carbonatisation et la chloritisation sont variables, dépendant de la présence ou non d'une altération hydrothermale avoisinante. Les sommets bréchifiés de ces coulées affleurent en de rares endroits, dont la rive sud du lac Ham, et permettent difficilement d'évaluer l'épaisseur individuelle de celles-ci. Duquette $(1976,1982)$ rapporte une épaisseur maximum de 60 mètres pour ces coulées, sur le flane nord du synclinal de Chibougamau. Une épaisseur moyenne variant entre 5 et 15 mètres est par contre probablement plus réaliste.

Les filons-couches gabbroiques compris dans cette formation sont fréquemment différenciés lorsque leur épaisseur le permet. Ils montrent alors une partie supérieure riche en phénocristaux de quartz (2 à $4 \%$ de la roche). C'est notamment le cas des filons-couches se retrouvant dans la partie centrale-sud du terrain et dans celui entourant le lac Ham. $D^{-}$autre part, de minces lits volcaniclastiques mafiques à felsiques, deépaisseur centimétrique à métrique, séparent les différentes coulées mafiques entre elles. Quelques rares minces lentilles pyriteuses s ${ }^{-} y$ retrouvent localement.

\subsubsection{Formation de Blondeau}

La Formation de Blondeau consiste en des sédiments volcaniclastiques principalement felsiques, des shales et silts verts, 
des basaltes localement variolaires, des pyroclastites felsiques, des tufs de composition variée et des agglomérats. Une tentative de corrélation entre notre section et celle du lac Geoff (Gobeil, 1982, communication personnelle; Lefebvre, 1986) située sur le flanc nord du synclinal de Chibougamau, basée sur la présence de coulées basaltiques variolaires, indique que notre région occupe un niveau stratigraphique similaire, soit la base de la Formation de Blondeau, à faible distance du contact avec la Formation de Gilman.

Dans la plupart des cas sur ce terrain, la partie inférieure de la Formation de Blondeau est dominée par des roches de nature sédimentaire, soit des lits grèseux, silteux, argileux et à intraclastes d’argilites (planches I (A), (B) et II (A)). Ces lithologies correspondent à celles décrites par Archer (1983, 1984), qui conclut à un environnement de bassin distal en émergence.

Sur le mont Bourbeau, ces faciès typiquement distaux sont mineurs. Ils sinterdigitent avec des lithologies devenant rapidement plus grossières et à aspect pyroclastique. Il sªgit de coulées monolithologiques constituées de cristaux libres de plagioclase, de fragments ponceux et souvent même de fragments rhyolitiques (planche II $(B)$ et (C)). Des lits et des lentilles contenant des fragments et blocs subanguleux monolithologiques se retrouvent plus spécifiquement dans le secteur du lac Devilliers (planche III (A)). Ces fragments et blocs, le plus souvent porphyriques et de compositions intermédiaires à felsiques (planche III (B) et (C)), ont jusqu’à 15 par 20 cm de côté. 
Ces lits ont des puissances variables, de 10 à $150 \mathrm{~cm}$ et contiennent parfois des fragments pyriteux ( $p$ lanche IV (A)) et des bombes volcaniques felsiques (planche IV (B)), recouverte par des sédiments de granulométrie fine et de composition felsique. Des sédiments volcanogènes remaniès, composés de fragments porphyriques en plagioclase et en quartz (provenant possiblement de 1'érosion de laves rhyolitiques ?) et des

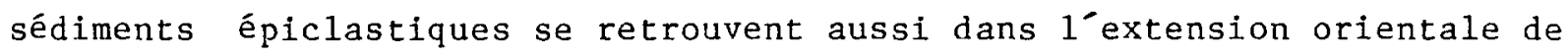
ces lithologies (planche V(A)).

Ces faciès plus grossiers signalent un environnement à caractère proximal dans le secteur immédiat du mont Bourbeau. A $1^{\prime}$ ouest du lac Line, ces faciès diminuent très rapidement en puissance et en étendue. L'occurrence de fragments pyriteux est pour sa part étroitement restreinte au secteur du lac Devilliers, au mont Bourbeau.

Les coulées de laves variolaires sont massives sur le mont Bourbeau (planche $V(B)$ ) et de massives à coussinées au sud du lac Larone. L'épaisseur individuelle de ces coulées varie de moins de un à plus de cinq mètres dans le secteur du lac Larone. Elles sont surmontées d’une mince couche de roche d’aspect pyroxénitique à gabbroíque, allant de 1 à 20 mètres d'épaisseur. Les contacts entre ces deux lithologies sont nets et localement paraissent intrusifs (planche VI (A)). Il pourrait $s^{\prime}$ agir d'un petit dyke d’aspect conforme ou plus vraisemblablement de la partie massive d’une coulée mafique.

Ces coulées de laves variolaires représentent possiblement les 
premiers signes $d^{*}$ un court épisode local de volcanisme mafique, signalé par des coulées du même type observées dans les environs de la trace axiale du synclinal de Chibougamau, dans le secteur nord-ouest de la carte. A cet endroit, des coulées de basaltes parfois variolaires, rarement coussinées, sont surmontées d'une zone bréchique et fragmentaire très épaisse, de 100 à 150 mètres, globalement de même composition (planche VI (B)). Cette grande épaisseur apparente de matériel bréchique pourrait être due d'autre part au pendage plus modéré des strates à proximité de la trace axiale du synclinal.

\subsection{Le Complexe de Cummings}

3.3.1 Le filon-couche de Roberge

La Formation de Blondeau est de façon générale 1'hôte des filons-couches du Complexe stratiforme de Cummings (Poitras, 1984). Sur le terrain, le filon-couche de Roberge s’est mis en place dans la partie supérieure de la Formation de Gilman. Son contact inférieur affleure très rarement, sauf dans le secteur au sud du mont Bourbeau. A cet endroit, la base de ce filon-couche est en contact avec une mince épaisseur (2 mètres) de sédiments volcaniclastiques intermédiaires à felsiques, faiblement cisaillée. Des basaltes affleurent immédiatement au sud.

L'épaisseur réelle de ce filon-couche est ici impossible à déterminer, celui-ci étant toujours tronqué à sa base ou à son sommet par une faille longitudinale. Son membre inférieur, quand présent en affleurement, montre un excellent litage rythmique, alternant 
graduellement d'une wherlite (planche VII (A)) et d'une pyroxénite, souvent poecilitique à une pyroxénite verte massive (planche VII (B)). Ces lits définissent des méga-cycles de 10 à 30 mètres d’épaisseur. A 1'intérieur même de ceux-ci, des cycles de plus petite échelle sont également discernables. En surface altérée, la pyroxénite poecilitique est grise-blanchâtre, tachetée de larges phénocristaux de pyroxène de 1 à $2 \mathrm{~cm}$ d’arète, pouvant représenter jusqu’à $60 \%$ de $1 \mathrm{a}$ roche. Ces phénocristaux contiennent des reliques de petits cristaux d’olivine maintenant totalement serpentinisés.

Le membre supérieur du filon-couche de Roberge se compose d'une zone dunitique relativement massive avec un litage magmatique peu à non développé. La serpentinisation y est souvent très intense, avec présence de minéraux amiantifères. Cette section du filon-couche de Roberge est très bien exposée le long du coté sud de la route qui mène aux dômes de radar, au sommet du mont Bourbeau et également au sud-ouest du lac Larone.

Cette subdivision du filon-couche de Roberge en deux membres principaux est proposée par Poitras (1984), à partir de ses études sur la section du lac Barlow et se trouve appuyêe par ces travaux de cartographie. Ce dernier décrit ainsi un membre inférieur bien lité, présentant une alternance de pyroxénite, de werhlite et de péridotite et un membre supérieur dunitique relativement homogène. Dans cette section, le filon-couche de Roberge possède une épaisseur de 440-450 mètres, ce qui pourrait également être une estimation fort plausible pour la 
région cartographiée. Ce filon-couche est couramment fortement magnétique et exige 1'utilisation de tables solaires ou d'autres techniques appropriées pour sa cartographie et la mesure des divers éléments structuraux.

3.3.2 Le filon-couche de Ventures

Ce filon-couche, lorsque sa base est non-tronquée par une faille longitudinale, repose directement sur le filon-couche de Roberge, soit par exemple au nord du mont Bourbeau et au sud-ouest du lac Larone. Par contre une mince bande discontinue de sédiments de la Formation de Blondeau marque ce contact, au sud-ouest du lac Line. Les sédiments y sont injectés de veinules de composition pyroxénitique. De facon générale, 1'épaisseur du filon-couche de Ventures varie de 100 à 140 mètres.

A la base de ce filon-couche, un membre pyroxénitique vert est

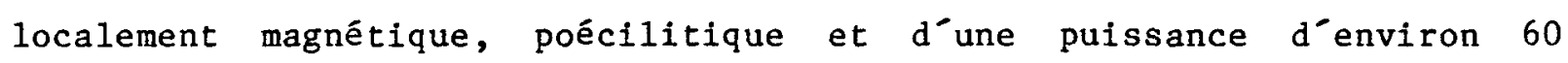
mètres. Il est constitué globalement d’un cumulat de phénocristaux de pyroxène (augite) ouralitisés de façon variable, ayant de .2 à .6 cm d'arète. Des bandes gabbroĩques, conformes au litage, peuvent se retrouver à tous les niveaux dans ce membre (planche VIII (A)). A certains endroits, notamment sur le mont Bourbeau, ces bandes conformes aboutissent à des dykes qui bréchifient la pyroxénite. Les parois de ces brèches sont localement faiblement pyritisées (planche VIII (B)). 
Vers le sommet du membre pyroxénitique, une foliation magmatique devient clairement évidente, soulignée par 1 'orientation préférentielle des cristaux aciculaires d’amphibole et de plagioclase ( $p l a n c h e ~ I X(A))$. Cette zone foliée, d'une puissance de un à trois mètres au maximum, marque générallement 1'apparition du plagioclase dans la roche. Cette zone souligne de plus la transition du membre pyroxénitique au membre gabbroĭque plus communément appelé "gabbro de Ventures". Ce dernier est facilement reconnaissable à sa texture gloméroporphyrique. Il s $\mathrm{s}^{\circ}$ agit $\mathrm{d}^{\circ} u n$ gabbro à texture ophitique composé en proportions égales, $40 \%-40 \%$, de plagioclases saussuritisés et de clinopyroxènes quasi entièrement ouralitisés en assemblage hornblende-actinote. Le plagioclase atteint une abondance maximum, $60 \%$, au sommet de ce membre. L'apatite est présente dans ce gabbro en quantité significative, de 1 à $2 \%$.

Le "gabbro de Ventures" est généralement surmonté d’une mince zone granophyrique, parfois discontinue, de 2 à 10 mètres d'épaisseur. Cette dernière zone est d’aspect plutôt massif et est par endroits faiblement silicifiée. Cette roche est fréquemment confondue sur le terrain avec un "tuf à cristaux" puisqu'elle est immédiatement surmontée par les strates de la Formation de Blondeau. La partie granophyrique, d'aspect blanc-crème en affleurement, est composée de 50 à $60 \%$ de plagioclases idiomorphes montrant localement une texture myrmékitique, de 10 à $15 \%$ d'amphibole et de chlorite et de 25 à $30 \%$ d'une matrice riche en quartz. Les phénocristaux de quartz même sont peu abondants.

Au nord-ouest du lac North, Smith (Smith et Allard, 1960) a 
identifié des dykes gabbroĩques felsiques d'une dizaine de mètres d’épaisseur sous les termes de "métadiorite et gabbro hybrides de bordure". La texture et la composition de ces dykes rappellent fortement les roches granophyriques du filon-couche de Ventures. Ces dykes se présentent à cet endroit immédiatement à la base du filon-couche de Roberge et sont apparemment conformes à la stratigraphie.

\subsubsection{Le filon-couche de Bourbeau}

Le filon-couche de Bourbeau est séparé du filon-couche de Ventures par une mince épaisseur, de 125 à 175 mètres, de strates appartenant à la Formation de Blondeau. L'épaisseur du filon-couche de Bourbeau varie de 500 à rarement plus de 600 mètres. Il se compose à sa base d'une pyroxénite massive ayant de 40 à 50 mètres d'épaisseur. L'orthopyroxène bronzite, rapporté par plusieurs auteurs (Allard,1976; Poitras,1984), nª pu être identifié de façon certaine dans cette partie basale. Un clinopyroxène fortement ouralitisé en actinote et serpentine et une faible quantité d'opaques sont les constituants majeurs de cette roche. La pyroxénite surmonte une zone de trempe de 2 à 3 mètres d’épaisseur, finement grenue, à texture gabbroĭque ( $p$ lanche IX (B)). Le contact inférieur avec les roches de la Formation de Blondeau est très net, conforme et ne montre pas d'évidence de métamorphisme de contact poussé (planche IX (C)).

A certains endroits, sur le mont Bourbeau et au sud du lac Larone, cette pyroxénite basale repose sur une roche péridotitique à dunitique de 
puissance variable, de 20 à plus d'une quarantaine de mètres ( $p l a n c h e X$ (A)). L'extension latérale de ces dernières lithologies est très restreinte, ce qui suggère une forme générale d’apophyse, reliée à la masse même du filon-couche. Ces apophyses bordent sur leur coté nord quelques strates typiques de la Formation de Blondeau. La position stratigraphique particulière de ces apophyses, nettement sous le niveau normal occupé par le filon-couche de Bourbeau, a probablement permis une différentiation magmatique et/ou une accumulation davantage marquée de minéraux mafiques à ces endroits.

De façon générale, le membre pyroxénitique de base est surmonté d’un gabbro leucocrate grossier, représentant près de la moitié de 1'épaisseur totale de ce filon-couche, soit de 225 à 250 mètres. Ce gabbro se compose de 40 à $50 \%$ d'hornblende, d’actinote, de pyroxènes ouralitisés et de 35 à $40 \%$ de plagioclases saussuritisés selon une intensité variable. Un litage rythmique se développe davantage vers le sommet de cette unité. Ce litage est constitué d'une alternance de minces 1its, d’épaisseur millimétrique à métrique, de plagioclases et d’amphiboles chloritisées. Ce membre leucocrate contient également des lentilles gabbroĭques de granulométrie grossière, de 20 à $40 \mathrm{~cm}$ de long par 10 à $15 \mathrm{~cm}$ de large, tapissées de cristaux d’amphiboles ayant couramment de 1 à $1.5 \mathrm{~cm}$ de longueur; des phénocristaux de quartz s’y observent aussi fréquemment.

Le ferrogabbro-ferrodiorite à quartz compose la partie supérieure du filon-couche de Bourbeau. Cette dernière unité, d'une épaisseur 
similaire à la précédente consiste en un assemblage de plagioclases ( 40 à 45\%), d'amphiboles (15 à 20\%), de rares pyroxènes ( 5 à 10\%) de quartz (10 à 15\%), d'opaques (le plus couramment pyrite et magnétite) et de minéraux d'altération (chlorite et épidote). Des dykes gabbroĨques leucocrates recoupent localement le mélanogabbro, entre autres sur le flanc nord du mont Bourbeau. Des lentilles gabbroĭques de granulométrie grossière (planche $X(B)$ ), à texture quasi pegmatitique, augmentent en importance et fréquence vers le sommet du ferrogabbro. Le contact supérieur de ce filon-couche avec la Formation de Blondeau, des laves basaltiques massives, est très net et affleure à faible distance au sud de la trace axiale du synclinal de Chibougamau, dans le coin nord-ouest de notre cartographie.

\subsection{Dykes}

3.4.1 Dykes porphyriques en plagioclase

Des dykes porphyriques en plagioclase et en quartz n’ont été rencontrés jusqu`à maintenant qu’à la base du filon-couche de Roberge (ou de Bourbeau ?), sur le mont Bourbeau (planche XI (A)). Ces dykes paraissent s'être mis en place de façon grossièrement conforme dans la base pyroxénitique du filon-couche présent. Ils contiennent des fragments cherteux localement. Une filiation entre ces dykes et un éventuel complexe volcanique felsique ou encore le stock tonalitique du lac Line est impossible à préciser davantage à ce stade-ci des travaux. Toutefois il apparait clair que ces dykes signalent une activité magmatique postérieure à la mise en place des filons-couches du Complexe de Cummings. 


\subsubsection{Dyke pyroxénitique}

Un dyke pyroxénitique, retrouvé à $1^{`}$ WNW du lac Ham, recoupe les sédiments pyriteux à graphiteux de la Formation de Gilman. Ce dyke, d`au moins un mètre de large, est composé de 15 à $20 \%$ de clinopyroxène complètement ouralitisé et d'une matrice riche en chlorite, épidote et carbonate, recristallisée et à grain fin (planche XI (B)). La texture de ce dyke ainsi que sa minéralogie rappelle les coulées de laves andésitiques porphyriques en pyroxènes de la Formation de Haüy, appartenant au Groupe d`Opémisca. Il montre cependant un contenu faible en potassium (annexe 2), contraire à ce qui est généralement observé dans les coulées volcaniques de cette formation ailleurs dans le district. Par contre Dubé (1983) dans les cantons de Lamarck et de Guettard et Allard (1982) dans le canton de Haüy rapportent des analyses de volcanites provenant de cette formation et qui sont localement anormalement pauvres en potassium. Une corrélation entre ce dyke et cette formation est ainsi proposée pour le moment.

\subsection{Le stock tonalitique du lac Line}

Ce stock plutonique recoupe les roches supracrustales archéennes dans le secteur du mont Bourbeau. Au sud de ce secteur, la tonalite se présente par contre sous la forme de grands dykes allongés, apparemment conformes à la stratigraphie et qui se sont mis en place dans le membre inférieur du filon-couche de Roberge. Ils isolent et bréchifient plusieurs segments pyroxénitiques. 
La partie nord de ce stock, affleurant en relief négatif, possède une forme en croissant avec un segment orienté ENE, en direction de la Belle Baie. Une faille délimite la bordure sud de ce segment et pourrait fort bien avoir dirigé partiellement la mise en place de ce stock. De plus, les textures présentées par ces roches ne montrent aucune fabrique métamorphique ou déformation majeure autres que celles en bordures des zones cisaillées. Ces caractéristiques lui confère un âge tardi à post-tectonique

Au moins trois faciès peuvent être distingués à $1^{\prime}$ intérieur de ce pluton. Le plus important est une leucotonalite massive (planche XII), composée de 40 à $55 \%$ de plagioclases idiomorphes zonés de .5 à $1 \mathrm{~mm}$ $d^{\prime}$ arète montrant une séricitisation et une chloritisation variables, croissantes en direction de la Baie du Cran Penché, à la périphérie est de ce stock. La matrice, cryptocristalline, consiste en un mélange de quartz (20 à 35\%) et de plagioclases (10 à 15\%) (planche XIII (A) et (B)).

Le second faciès affleure plus rarement. Il s’agit d'une syénite avec phénocristaux d’amphiboles aciculaires ayant jusqu’à $1.5 \mathrm{~cm}$ de long (planche XIV (A)). Cette syénite se retrouve sous la forme d'un dyke $d^{\top}$ une puissance de six à sept mètres, introduit dans une roche $d^{`}$ aspect pyroxénitique dans la galerie d'exploration "Be1-3" (à 1'ouest du lac Line) et en amas de forme quelconque dans la leucotonalite (planche XII). Des veinules d’axinite et d’épidote, de cinq mm à deux cm de large, sont 
observées recouper alors la leucotonalite et la syénite. Une syénite

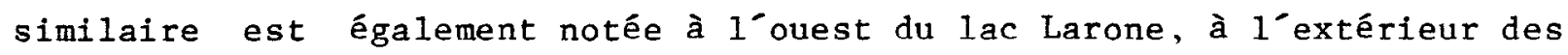
terrains cartographiés ici, injectée à la base du filon-couche de Bourbeau (Pilote, 1984 et 1986).

Le troisième faciès est plus marginal. Il se présente en bordure des grands dykes tonalitiques observés dans la moitié sud des terrains cartographiés. Il $\mathrm{s}^{\top}$ agit d'une diorite à texture gabbroĩque, localement foliée et qui passe graduellement sur deux ou trois mètres à une leucotonalite massive (planche XIV (B)). La foliation est généralement parallèle aux contacts.

Smith (Smith et Allard, 1960) a reconnu le caractère distinct de ces deux derniers faciès en les regroupant dans une unité identifiée sous les termes de "métadiorite, métagabbro hybride de bordure et laves feldspathiques". Seul 1'appellation "laves feldspathiques" serait à écarter. D'autre part, cette texture gabbroĭque ne ressemble à aucun des faciès des filons-couches du complexe de Cummings. Les larges dykes tonalitiques rencontrés au sud du mont Bourbeau, selon la cartographie de Smith (Smith et Allard, 1960) et notre interprétation, devraient se poursuivre en direction est, à $1^{\prime}$ extérieur de la zone couverte par cette étude.

Au sud de la Baie du Cran Penché, le stock tonalitique contient de nombreuses inclusions pyroxénitiques de tailles diverses, d’échelle centimétrique à mètrique, anguleuses, appartenant vraisemblablement au 
filon-couche de Roberge. Des dykes tonalitiques discordants, de quelques centimètres à une cinquantaine de mètres de large, se retrouvent aussi dans les roches archéennes encaissantes, soit par exemple à l'est du lac Line dans le filon-couche de Bourbeau et dans le filon-couche de Roberge, au sud du mont Bourbeau. Le métamorphisme de contact autour de ce pluton est en général faible. Là où le pluton est le plus large, autour du lac Line, le métamorphisme se remarque jusqu’à une centaine de mètres dans les volcaniclastites de la Formation de Blondeau et à un degré moindre dans les différents filons-couches du complexe de Cummings. Les roches encaissantes sont alors partiellement silicifiées, chloritisées et montrent couramment un aspect de cornéenne (planche XIV (C)).

3.6 Formation de Chibougamau

Toutes les lithologies archéennes précédentes sont recouvertes en discordance par des lambeaux protérozoĩques (Long,1974) de conglomérats, d'arkoses, de grès et de tillites d’origine glaciaire. Déjà bien auparavant, Barlow et al. (1911) et Retty (1929) soupconnaient le caractère glaciaire et tardif de ces dépôts; ils avaient de plus noté leur similitude avec des sédiments observés en Ontario dans la région de Cobalt, sédiments réputés d’âge Huronien à cette époque. Mawdsley et Norman (1935) ont pour leur part rapporté la découverte d'un boulder strié dans les roches protérozoìques sur le mont Bourbeau et soupçonnaient aussi l'origine glaciaire de ces dépôts. Ce sont également eux qui ont identifié ces roches sédimentaires sous le nom de "Séries de Chibougamau", ce terme devenant plus tard Formation de Chibougamau. 
Sur le mont Bourbeau, ces séquences protérozoĩques possèdent un léger pendage de $10^{\circ}$ à $15^{\circ}$ vers le sud. A cet endroit, un conglomérat hétérolithique, composé de fragments subarrondis de granitoĭdes et de volcanites, recouvre les roches supracrustales. La dimension de ces fragments augmentent vers le sommet. L'épaisseur du couvert protérozoíque atteint de 25 à 50 mètres sur le sommet du mont Bourbeau. La différence d'élévation du niveau d'érosion archéen atteint plus de deux mètres (Gobeil, dans Allard et al., 1979). Un forage à la base de ce mont a également permis d’estimer une épaisseur apparente de 275 mètres de sédiments protérozoĩques (Graham, 1967).

Au sud du lac Larone, la couverture de sédiments atteint une épaisseur d'environ 60 mètres d'après un trou de forage (Cronsilver, 1957). Un conglomérat de base de 6 à 7 mètres d'épaisseur repose alors sur une mince bande de sédiments à grain très fin. Le reste de la section se compose d'une alternance de bandes conglomératiques et de tillites de puissances variées. 
CHAPITRE 4

GEOLOGIE STRUCTURALE

4.1 Plis

Duquette (1970, 1976 et 1982) est le premier qui a identifié le synclinal de Chibougamau. Selon celui-ci la trace axiale de cette structure, d'orientation est-nord-est, se retrouve dans le coin nord-ouest de notre cartographie, à 1'intérieur d’un niveau de basalte appartenant à la Formation de Blondeau. La plus grande partie de nos terrains occupe le flanc sud de ce synclinal. Sur ce flanc, les stratifications observées sont également orientées est-nord-est et les polarités ont leur regard régulièrement vers le nord. Les pendages sont abrupts vers le nord à subverticaux. La trace de la schistosité régionale S2, qui est identifiée par 1'aplatissement caractéristique des textures volcaniques et sédimentaires, est d’autre part généralement parallèle à la direction présentée par la stratification, à 1'extérieur de la zone

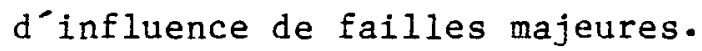

Outre ce synclinal, aucun autre pli majeur ne semble perturber

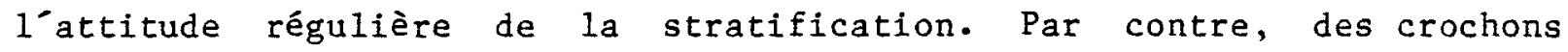
d'entrainement sont observés localement le long de certaines failles directionnelies importantes. C'est ainsi le cas par exemple sur le mont Bourbeau, dans le membre supérieur du filon-couche de Roberge. Cette situation sera traitée plus à fond dans la section qui suit. 
4.2 Failles

4.2.1 Introduction

\begin{abstract}
Cette région est affectée très inégalement par différents réseaux de failles. Certaines lithologies semblent davantage propices au développement de certaines cassures. De plus, des fracturations d'orientations particulières semblent se présenter dans des périodes de temps données, que ce soit avant, pendant ou après 1'orogénèse kénoréenne. Ces critères, de même que la composition des différents indices minéralisés se retrouvant associés à différentes failles, nous

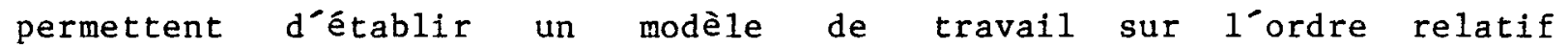
d’apparition des réseaux de failles majeurs. Les minéralisations qui sont assignées à chaque phase de fracturation seront discutées dans une section ultérieure. Par ordre chronologique d'occurrence, ces failles sont soit d’âge syn-volcanique, syn-kénoréen et tardi ou post-kénoréen (tableau 2).
\end{abstract}

\title{
4.2.2 Failles syn-volcaniques
}

Ces failles sont orientées $\mathrm{N}-\mathrm{S}$ à NNE et rarement NNW. Elles possèdent des pendages abrupts et affectent principalement la base du filon-couche de Bourbeau et le sommet du filon-couche de Ventures, dans le secteur du mont Bourbeau. A ce dernier endroit, la présence d’un centre volcanique felsique est d’ailleurs soupçonnée. Les roches de la Formation de Blondeau ne montrent pas de développement appréciable de ce type de faille, sauf dans le secteur du lac Devilliers. Sans doute 


\begin{tabular}{|c|c|c|c|c|}
\hline \multicolumn{2}{|r|}{ Orientation } & $\begin{array}{l}\text { Décrochement apparent, } \\
\text { amplitude }\end{array}$ & Age relatif & Exemple $d^{-}$emplacement \\
\hline 1 & NNE & $\begin{array}{l}\text { senestre et dextre, de } \\
\text { type effondrement (?), } \\
\text { de } 0 \text { a } 150 \mathrm{~m} \text {. }\end{array}$ & syn-volcanique & $\begin{array}{l}\text { Mont Bourbeau, le secteur } \\
\text { du lac Devililers; le lac } \\
\text { Berrigan (prospect Taché) }\end{array}$ \\
\hline 2 & EW a ENE & $\begin{array}{l}\text { mouvement inverse } \\
\text { dextre présumé, } \\
\text { amplitude indéfinie }\end{array}$ & pré a syn-Kénoréen & fatile du lac cummings \\
\hline \multirow[t]{4}{*}{3} & NNE $\quad(R)$ & senestre, $200 \mathrm{~m}$ & & $\begin{array}{l}\text { affecte la région au } \\
\text { complet }\end{array}$ \\
\hline & NNW a NW $\left(R^{-}\right)$ & dextre, $\quad 100 a 200 \mathrm{~m}$ & tardt a post-Kénoréen & $\begin{array}{l}\text { au sud du lac Gwillim, } \\
\text { relativement rare }\end{array}$ \\
\hline & ENE a E-W (P) & senestre, $\times 100 \mathrm{~m}$ & (Dimroth et al., 1984a) & $\begin{array}{l}\text { sur le flanc sud du } \\
\text { synclinal de Chibou- } \\
\text { gamau, peu fréquente }\end{array}$ \\
\hline & NE a ENE (D) & senestre, $2 d 3 \mathrm{~km}$ & & la fallle du lac Gwillim \\
\hline \multirow[t]{2}{*}{4} & NNE $\gtrsim N E$ & dextre, $\quad 100 a 300 \mathrm{~m}$ & $\begin{array}{l}\text { post-tectonique, } \\
\text { Protérozolques }\end{array}$ & $\begin{array}{l}\text { en bordure des lambeaux } \\
\text { protérozotques }\end{array}$ \\
\hline & ENE & $\begin{array}{l}\text { mouvement inverse } \\
\text { senestre (?) présumé, } \\
\text { amplitude Indéfinie }\end{array}$ & $\begin{array}{l}\text { (Duquette, } 1976 \text { et 1982) } \\
\text { (Dimroth et al., 1984a } \\
\text { et 1985b) }\end{array}$ & la fallle du lac North \\
\hline
\end{tabular}

Tableau 2 - Chronologle relative des divers réseaux de fallles, modèle de traval, réglons du mont Bourbeau et du lac Berrigan.

Note: Chaque nouvel Eplsode de fracturation est susceptible d'avolr réactivê certalnes fallles précédentes, d'orientations favorables. 
faut-il y voir un comportement mécanique différent entre un sédiment partiellement consolidé et une roche intrusive cristalline. Ces failles sont recoupées ou délimitées en extension par certaines lithologies ou encore par d’autres cassures décrites dans les sections qui suivent.

Ces failles réputées d’âge syn-volcanique atteignent rarement plus d'une dizaine de mètres de long à la base du filon-couche de Bourbeau et de 40 à 50 mètres dans le filon-couche de Ventures. A 1'intérieur de ce dernier filon-couche, des dykes gabbroíques d'orientation NNE empruntent ces cassures et recoupent les faciès pyroxénitiques sousjacents (planche VIII (B)). Aucun décalage majeur n'est observé dans les lithologies encaissantes de chaque coté de ces cassures. De par leur étendue et leur configuration, ces failles ne s’associent à aucun système de contrainte majeur reconnu dans la région.

Dans le secteur du lac Devilliers (figure 9, cf. chap. 5), de façon résumée, ces failles sont de courte extension et ont permis le passage des fluides hydrothermaux et la déposition de minéralisations volcanogènes stratiformes. Ces cassures présentent des décrochements de directions variables, dextres et sénestres. Les propagations de ces cassures et des minéralisations ont été partiellement entravées par 1'épanchement subséquent de coulées basaltiques variolaires.

Par ailleurs, la faible densité d’affleurements à la base du filon-couche de Roberge, dans les environs du mont Bourbeau, nous empèche d'observer à cette position stratigraphique 1'occurrence attendue de ce 
type de faille.

Par contre du côté ouest, à $1^{\prime}$ extérieur de notre cartographie et à moins de $4 \mathrm{~km}$ du mont Bourbeau nous retrouvons dans les environs du lac Berrigan un très bon exemple de ces failles (Pilote et al., 1984a; Pilote, 1986). D'une façon sommaire, le filon-couche de Roberge est affecté à cet endroit par des failles syn-volcaniques orientées NNE. Les pendages de ces failles sont subverticaux ou abrupts vers le SE et elles délimitent très bien les bordures d'un bloc de la Formation de Blondeau, enfoncé partiellement à 1'intérieur des filons-couches de Ventures et de Roberge. Cette situation reflète vraisemblablement, selon ces auteurs,

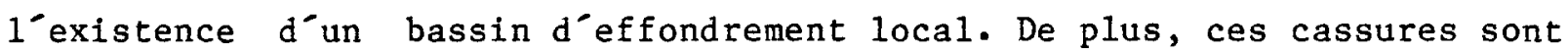
localement porteuses de minéralisations elles-mêmes d’âge volcanique (Thorpe et al., 1981 et 1984; Guha, 1984). Les extensions nord de ces failles et des minéralisations adjacentes sont tronquées par une faille inverse majeure de direction E-W, la faille du lac Cummings.

Ailleurs dans la région étudiée, ce type de courte cassure śest avérée relativement rare.

\subsubsection{Failles syn-tectoniques}

Il s'agit de failles directionnelles, d’orientation générale E-W, qui sont sub-parallèles ou à angle faible en surface aux lithologies encaissantes (Dimroth et al., 1983 et 1985b; Daigneault et Allard, 1984). Ces cassures, présumément des failles normales à leur origine, sont 
responsables de grands chevauchements et amènent de façon plus spécifique sur nos terrains la répétition de certaines lithologies qui conservent toujours le même sommet stratigraphique, en direction nord. Une de ces failles, un cisaillement majeur, traverse d`ailleurs complètement le secteur d’étude. Elle se présente à 1'ouest du lac Larone, se continue sur le mont Bourbeau et en direction est sous les eaux de la Baie du Cran Penché pour finalement se poursuivre au sud de la mine Norbeau, tout

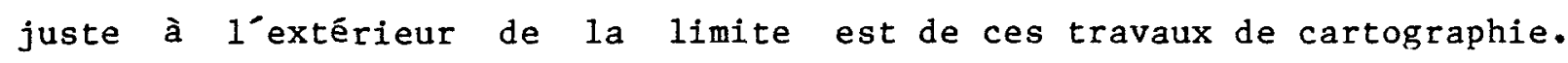
Cette faille répète tout le flanc sud du synclinal de Chibougamau, ceci incluant le Complexe de Cummings au complet.

Cette cassure met en contact sur son coté nord le filon-couche de Roberge et du coté sud le filon-couche de Bourbeau (figure 5). Le cisaillement principal prend place du coté du filon-couche de Roberge et des petites fractures parallèles $s^{\prime} y$ remarquent sur une distance plus grande, de 20 à 40 mètres, que du coté du filon-couche de Bourbeau. Cette situation est notamment observée à la galerie d'exploration "Be1-3" (chapitre 5). Ce cisaillement possède un pendage subvertical à abrupt, $85^{\circ}$, vers la nord. Des stries, révélatrices du sens du mouvement, n`ont pu malheureusement être observées en bordure de cette zone. Néanmoins la topographie actuelle suggère que le bloc situé au sud de la trace du cisaillement principal a surmonté le bloc nord, dans un mouvement typique de faille inverse.

Allard et Cimon (1972; tiré de Allard, 1976) ont noté une faille inverse similaire dans les environs du mont Cummings dans le canton de 


\section{SECTION SIMPLIFIÉE DE LA PARTIE}

\section{OUEST DU MONT BOURBEAU}

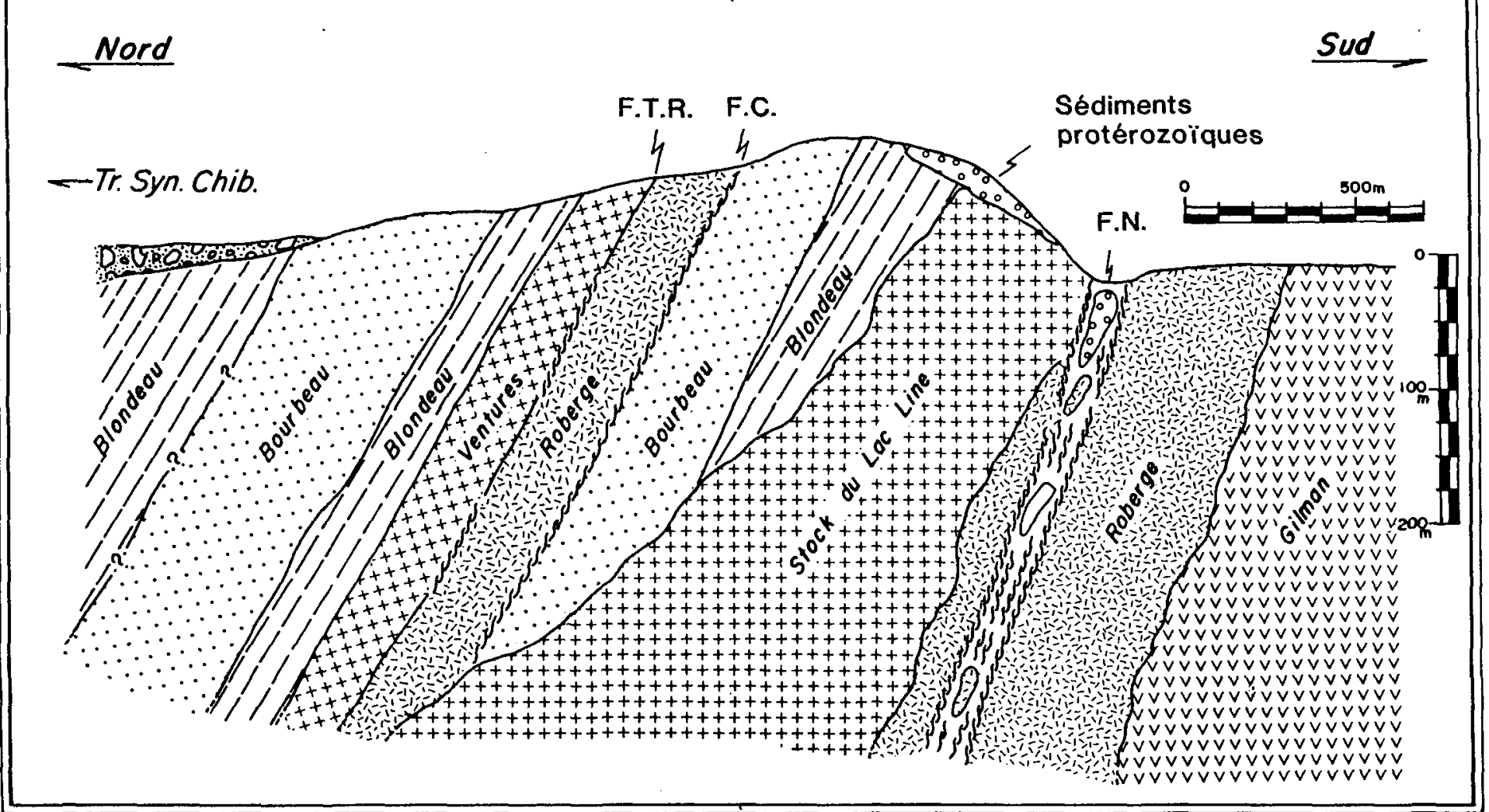

Figure 5. Section simplifiée de la partie ouest du mont Bourbeau. F.T.R. = la faille du toit du filon-couche de Roberge, F.C. = la fatlle du lac Cummings et F.N. = la faille du lac North. 
Roy et lui ont assigné le nom de "Faille du synclinal de Chibougamau". La structure en chevauchement rapportée par nos travaux de cartographie pourrait fort bien $s^{\prime}$ identifier à cette faille puisque cette dernière se retrouve dans son prolongement attendu. Par la suite, Daigneault et Allard (1984) ont suggéré le nom de Faille du lac Cummings à cette structure. Nous avons retenu pour le moment cette dernière appellation et $1^{\text {' }}$ avons décernée à la faille inverse reconnue sur nos terrains. Vers $1^{\prime}$ ouest, cette faille se poursuit au molns jusqu’au lac Elaine (Pilote, 1986) et se bute fort probablement sur la faille du lac Gwillim.

Dans la partie centrale du synclinal de Chibougamau, ces cassures de direction $E-W$ se manifestent fréquemment à 1 'intérieur du membre supérieur dunitique-péridotitique, davantage serpentinisée, du filon-couche de Roberge (Duquette, 1970, 1976, 1982; Allard, 1976; Pilote, 1983). Duquette (1976) a observé que ce style de faille borde régulièrement le sommet de ce filon-couche et leur a attribué ainsi le nom de "Roberge Roof Fault" (ou Faille du Toit du Filon-couche de Roberge). De façon plus détaillée sur le terrain cartographié, le membre supérieur du filon-couche de Roberge est faillé de façon très discontinue et variable en intensité. Ceci suggère que la trace véritable de la faille inverse majeure occupe des niveaux également variables dans ce membre dunitique supérieur et non nécessairement son sommet au sens stricte (figure 5).

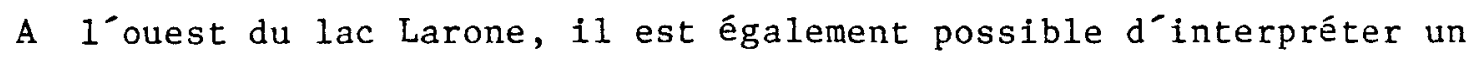
mouvement apparent horizontal dextre sur la trace de cette faille par 
1 'observation des schistositês, en forme de "S", et des linéations.

En bordure nord de la trace inférée de la faille du lac Cummings se retrouve sur le mont Bourbeau un pli d’entrainement mineur très serré ayant $1^{\text {'aspect }} \mathrm{d}^{\text {`u }}$ an anticlinal (planche XV). Ce pli, subvertical et plongeant de $47^{\circ}$ en direction ESE, affecte une péridotite serpentinisée prenant place dans la partie supérieure du filon-couche de Roberge. Ces caractéristiques démontrent que la composante horizontale du mouvement total était dextre, le bloc sud chevauchant alors le bloc nord. Ces plis d'entrainement représentent possiblement, à une échelle réduite, une structure anticlinale analogue à celle que Duquette (1970, 1976 et 1982) a observée ailleurs dans le filon-couche de Roberge, le long du synclinal de Chibougamu et $q u^{\top i}$ a identifiée sous le nom d"Anticlinal du Filon-couche de Roberge". La proximité de cisaillement de direction E-W dans les environs de ces structures anticlinales n’est pas discutée par Duquette mais serait fortement à envisager.

Une seconde faille inverse, d'extension mineure, est encaissée dans les sédiments volcaniclastiques de la Formation de Blondeau, au sud du lac Larone.

Le mouvement inverse présenté par ces failles et le fait que celles-ci soient recoupées par d'autres réseaux de cassures indiquent qu'elles sont associées de façon précoce au système en compression de direction $\mathrm{N}-\mathrm{S}$ responsable des structures synclinales majeures (Dimroth et al., 1983, 1984a et 1985b; Daigneault et A1lard, 1984). 


\subsubsection{Ceinture de cisaillement du lac Gwillim}

Cette ceinture de cisaillement a été identifiée suite aux récents travaux de Dimroth et al. (1984a). Selon les hypothèses de travail présentées, il est proposé que cette ceinture soit responsable de la création de quatre différents réseaux de failles (figure 6). Ces réseaux, selon la nomenclature proposée et utilisée par Tchalenko (1968), sont orientés NNE ( $R$, les cisaillements de Riedel), NNW à NW ( $R^{-}$, les cisaillements de Riedel conjugués), ENE à E-W ( $P$, les cisaillements chevauchants) et finalement $N E$ à $\operatorname{ENE}$ (D, le cisaillement majeur). Ces réseaux de cassures se sont succédés les uns aux autres dans un court interval de temps. Cette ceinture est d’âge tardi-archéen. La contrainte de compression régionale, instigatrice de ces réseaux, aurait été orientéée NNE $\left(010^{\circ}\right)$. Certaines de ces failles, dont celle du lac Gwillim, sont demeurées actives même au Protérozoǐque.

Les interprétations et hypothèses obtenues (figure 7) à partir de la mesure des différents éléments structuraux recueillis sur le terrain sujet à notre étude (Pilote et al., 1984a; Pilote, 1986), concernant la distribution et $1^{\prime}$ orientation de certains réseaux de failles, se sont avérées d’autre part en accord avec les résultats préliminaires dégagés par Dimroth et al. (1984a).

Sur ce terrain, les failles " $R$ ", d'orientation NNE, sont particulièrement bien développées dans la zone comprise entre le mont 


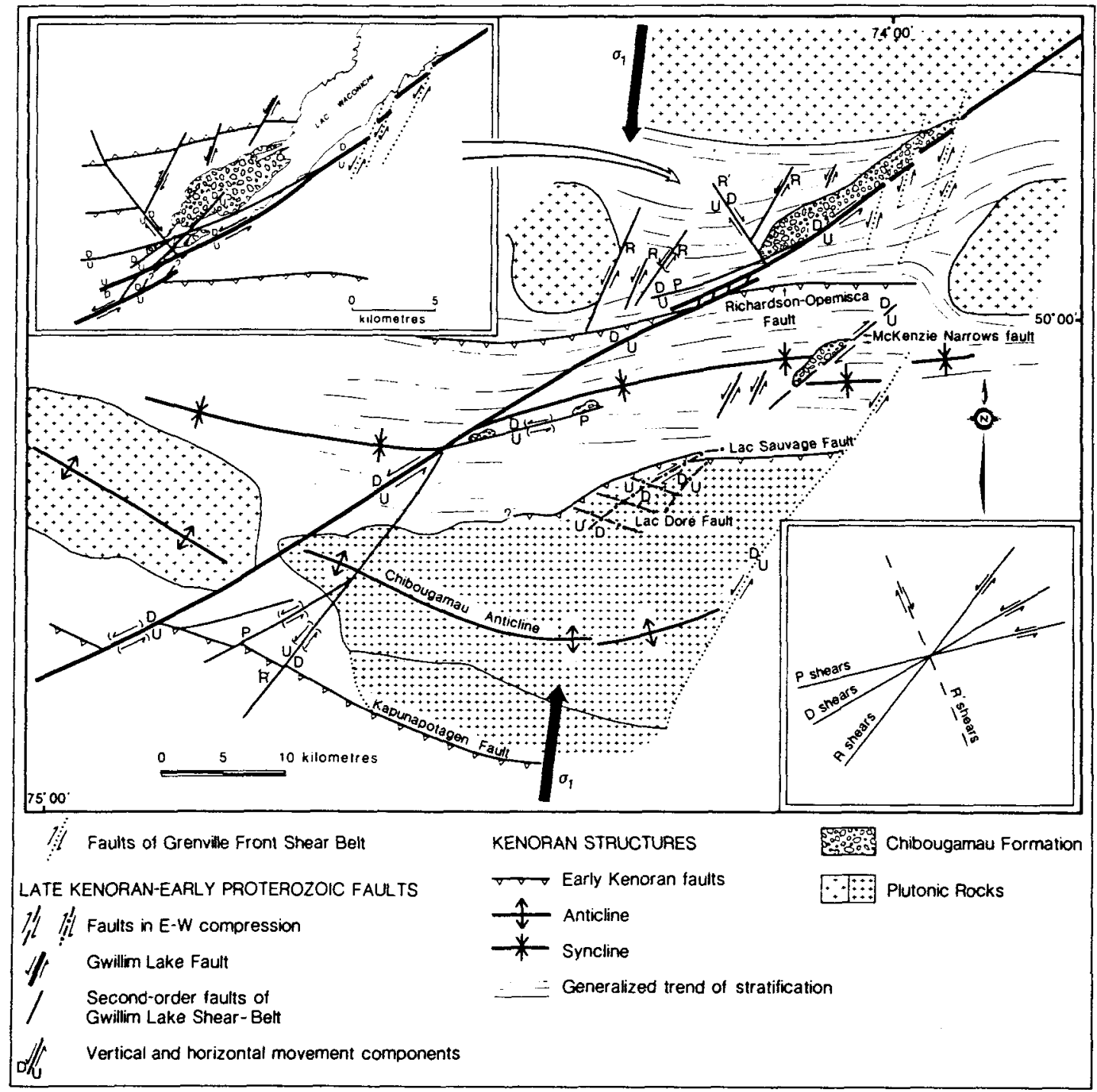

Figure 6. Distribution et répartition des failles et des cisaillements dans les environs de la ceinture de cisaillement du lac Gwillim. LLF = Faille du lac Laura; CCF = Chibougamau Copper Fault; SLF = Faille du lac Scott. $D^{\prime}$ après les travaux préliminaires tirés de Dimroth et al. (1984a). 


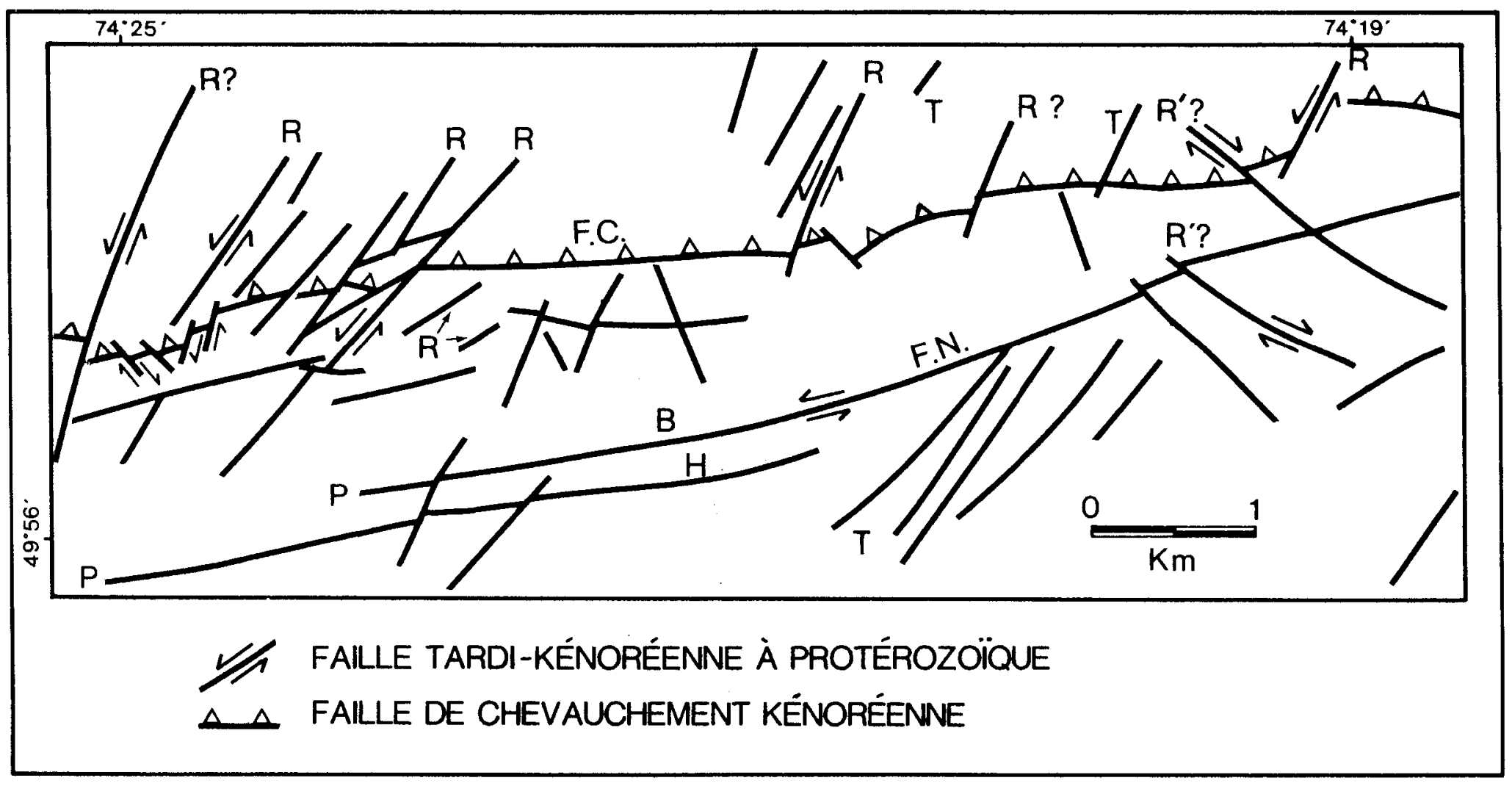

Figure 7. Distribution et répartition des failles et cisaillements dans les régions du mont Bourbeau et du lac Berrigan. Les fallles nommées par les termes $R^{\prime}$, $R$ et $P$ se rattachent à la ceinture de cisaillement du lac Gwillim (C.C.G.) (Dimroth et a1., 1984a). Celles identifiées par $T$ sont d'âge protérozol̃que. Certaines failles, $R$ ? et $R^{-}$?, représentent possiblement une réactivation protérozỡque de failles kénoréennes. Les sens de décrochements sont alors parfois inversés. Le mouvement des blocs, haut (H) et bas (B), de chaque côté de la faille du lac North (F.N.) est déduit des relations établies avec la C.C.G. et ne tient pas compte des mouvements protérozoĩques ultérieurs. F.C. = faille du lac Cummings. 
Bourbeau et le lac Larone. Leur fréquence est croissante en direction ouest, vers le lac Gwillim et décroissante vers 1'est. Elles demeurent toutefois toujours présentes sur toute l'étendue du secteur. Le pendage est abrupt, le plus fréquemment dirigé vers $1^{\prime}$ 'est. Ces cassures recoupent la trace d'une faille inverse, celle du lac Cummings. Elles ont de plus réactivé certaines failles syn-volcaniques N-S/NNE précoces (Pilote, 1984 et 1986; Landry, 1984 ).

Ces cassures NNE sont d'extension appréciable, c’est-à-dire qu'elles affectent le Complexe de Cummings en entier et les roches de la Formation de Blondeau. Les décrochements apparents horizontaux observês

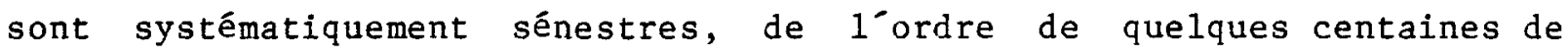
mètres.

Les failles " $R^{-1}$, de direction WNW à NW, sont peu fréquentes dans le secteur étudié. Quelques unes ont été retrouvées au sud du lac Larone. Les décrochements apparents sont dextres et courts, inférieurs à une centaine de mètres. Le pendage est abrupt vers le NE. $D^{\prime}$ autres cassures d’orientations similaires sont interprétées recouper le stock tonalitique du lac Line, au sud de la Baie du Cran Penché (carte hors-texte). Le sens des décrochements est par contre encore mal défini. Le stock intrusif recoupe pour sa part une faille inverse et plusieurs failles NNE ("R"). Ce stock est par contre recoupé au sud du mont Bourbeau par une faille protérozoĩque, ce qui indique à tout le moins pour celui-ci une mise en place tardi-kénoréenne. 
Les cisaillements "P", d'orientation ENE, sont peu fréquents sur le terrain. Ils se remarquent plus particulièrement au sud du lac Larone. Les décrochements apparents sont systématiquement senestres et courts, inférieurs à une centaine de mètres de long. Le pendage est abrupt vers le SE. Egalement certaines failles d'orientations similaires, mais montrant des extensions beaucoup plus grandes et des évidences de mouvements plus tardifs, post-Kénoréens, pourraient avoir été instaurées lors de cette période.

Les cisaillements "D", de direction NE à ENE, ne sont pas observés comme tel sur ce terrain. La faille du lac Gwillim, située à moins de 6 $\mathrm{km}$ à 1 -ouest du mont Bourbeau, est la manifestation principale et ultime de ce réseau (Dimroth et al., 1984a). Des failles d'orientations similaires se retrouvent par contre en bordure du lac Elaine, immédiatement au sud du lac Gwillim (Pilote, 1986).

régulièrement

lles protérozoĩques

Une activité tectonique post-kénoréenne s'est fait sentir jusqu’à cette période et même au-delà. La manifestation la plus évidente en est la présence de cinq lambeaux de sédiments protérozor̃ques, alignés sur le côté nord d’un linéament topographique, une faille inférée d’au moins $11 \mathrm{~km}$ de long. Cette zone de cisaillement, d'orientation ENE-WSW, s'étend du lac Elaine à 1'ouest (Pilote, 1986), à la limite des cantons McKenzie-Barlow et jusqu'à la Baie du Cran Penché au moins du coté est. A ce dernier endroit, cette faille recoupe le stock du lac Line, mais les 
évidences manquent pour situer son prolongement est avec plus de certitude. Le pendage de cette faille est de 60 à $70^{\circ}$ vers le nord (Graham, 1967), déterminé d'après des forages enfoncê au sud du mont Bourbeau (figure 5). Les sédiments protérozoĩques ont étê préservés du coté nord de cette structure, ceci signifiant que $1^{\prime}$ accumulation de ces dépôts a pris place dans une dépression préexistante ou encore qu'il y a eu enfoncement de ce dernier bloc, après la déposition protérozoĩque. La position actuelle de ces sédiments, au sommet d'une colline, implique vraisemblablement un ou des mouvements post-protérozoĩques.

Ainsi, au sud du mont Bourbeau, Graham (1967) rapporte que des forages ont intercepté à une profondeur réelle d'au moins 275 mètres des roches protérozoìques, cisaillées de façon variable, sous les eaux de la Belle Baie. Cette situation peut être expliquée par le cisaillement

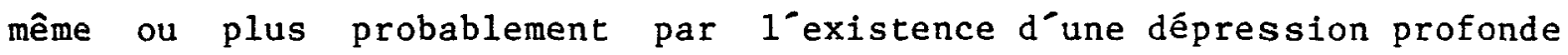
dans la paléo-surface d'érosion, avant la déposition de ces sédiments (Smith, dans Smith et Allard, 1960; Graham, 1967). Cette dépression peut $s^{` e}$ être produite de trois manières, soit par une faille inverse précoce (syn-tectonique), par un cisaillement ENE-WSW ( du type "P"), globalement contemporain de la zone de cisaillement du lac Gwillim (Dimroth et al., 1984a) ou encore par une conjonction de ces deux mécanismes. Finalement des mouvements tectoniques ultérieurs, d’âge protérozoĩque, le long des cassures ENE préexistantes, auraient assuré la continuité et $1^{-}$envergure de ce linéament, que nous avons identifié sous le nom de "Faille du lac North". Un ou des mouvements inverses, le long de ce linéament, deviennent alors nécessaires pour expliquer 1'emplacement 
actuel de ces sédiments au sommet de cette série de collines.

L’influence de ce linéament sur les roches encaissantes est variable, dépendant du type de lithologie présent. Ainsi, au sud du lac North et vers 1'ouest, la Formation de Gilman met en évidence un cisaillement, de pendage subvertical, sur une épaisseur de près de 100 mètres. La roche a souvent 1'aspect d'un "schiste à papier", la carbonatisation et la schistositê sont très intenses en direction du contact entre les laves et le filon-couche de Ventures. Le cisaillement majeur se situe par contre au centre du lac et affecte la base du filon-couche de Ventures. L'absence du filon-couche de Roberge, au sud du lac North, appuie ainsi 1 'idée que ce cisaillement protérozoĩque possède également une composante verticale appréciable en chevauchement.

Les cinq lambeaux protérozoĩques protégés par la faille du lac North et observés sur les sommets de collines montrent, de $1^{\prime}$ ouest vers 1`est, une épaisseur régulièrement décroissante, minimale sur le mont Bourbeau. Ceci suggère que le mouvement ascendant du bloc situé au nord de ce linéament était d’une intensité inégale, beaucoup plus accentué en direction est que ouest. L'extension ouest de ce linéament sªppuie vraisemblablement sur la faille du lac Gwillim (Pilote, 1986).

Sur le mont Bourbeau, les sédiments protérozoĩques sont recoupés par quelques failles NNE. Ces failles montrent des décalages apparents dextres, inférieurs en envergure à une cinquantaine de mètres. Le sommet du filon-couche de Bourbeau, au nord des dômes du radar ainsi que toute 
la demie est du terrain étudié, présentent localement ce même type de faille. La longueur de ces cassures est variable, de 100 à plus de 400 mètres. Elles ont très bien pu localement réactiver certaines failles de direction NNE à NE ("R") et ENE ("D") de la ceinture de cisaillement du lac Gwillim, ce qui rend encore plus malaisée la distinction entre ces différents types de failles, en 1'absence d'horizons-repères fiables. 
CHAPITRE 5

MINERALISATIONS

\subsection{Introduction}

Les différents indices minéralisés rencontrés dans cette région seront décrits dans un premier temps par ordre stratigraphique ascendant, soit ceux se retrouvant dans la Formation de Gilman jusqu’à ceux inclus dans le stock tonalitique du lac line et en second lieu d’après le type de roche qui les encaisse. Des considérations sur leur mode de mise en place et leur chronologie seront données au chapitre suivant, en tenant compte de leurs caractéristiques pétrographiques, minéragraphiques et structurales. Un chiffre encerclé, ex.:1), réfère à un site localisé sur la carte en pochette (échelle 1:5 000). Le tableau 3 résume les teneurs des principaux indices visités.

\subsection{Formation de Gilman \\ 5.2.1 Minéralisations stratiformes}

Cette formation contient quelques rares lits minéralisés en pyrite, pyrrhotine et très rarement en chalcopyrite. Ces lits ont une configuration stratiforme et sont disposés de façon très irrégulière dans des sédiments entre les différentes coulées basaltiques. L'épaisseur de ces lits n'excède jamais $1^{\prime}$ échelle millimétrique et leur extension

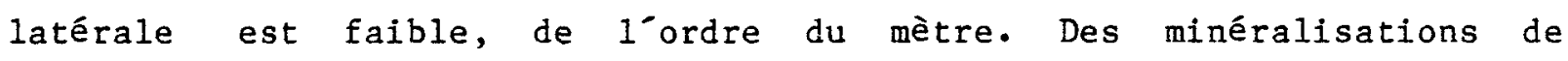
composition similaire se retrouvent aussi en interstices de coussins 
FORMATION DE GILMAN

-Indice du lac Ham

FORMATION DE BLONDEAU

$\begin{array}{cccccccc}\mathrm{Cu} & \mathrm{Zn} & \mathrm{N} 1 & \mathrm{~Pb} & \mathrm{Cr} & \mathrm{Co} & \mathrm{Au} & \mathrm{Ag} \\ 2.177 & 110 & 160 & <.5 & 2 & 650 & 90 & 26 \mathrm{~g} / \mathrm{t} \\ & & & & & & & \\ .117 & 87 & 65 & 46 & 19 & & <.5 \mathrm{~g} / \mathrm{t}<.5 \mathrm{~g} / \mathrm{t} \\ .147 & 170 & 590 & 55 & 16 & & <.5 \mathrm{~g} / \mathrm{t}<.5 \mathrm{~g} / \mathrm{t} \\ .27 \% & 910 & 170 & 560 & .237 & 660 & 80 \quad 59 \mathrm{~g} / \mathrm{t} \\ .257 & 100 & & 5 & & & <.5 \mathrm{~g} / \mathrm{t}<.5 \mathrm{~g} / \mathrm{t}\end{array}$

-Fragments pyriteux, au SE du lac Devililer

-Lentille substratiforme de Po, près du sommet du filon-couche de Ventures - Contact cisalllé et minelisé entre sédiment et ba se du f.-c. de Bourbeau - Lentilles stratiformes pyriteuses, au sud du lac Larone

COMPLEXE DE CUMMINGS

\begin{tabular}{|c|c|c|c|c|c|c|c|}
\hline $.37 \%$ & $28.4 \%$ & 110 & $.20 z$ & 19 & & $2.3 \mathrm{~g} / \mathrm{t}$ & $27 \mathrm{~g} / \mathrm{t}$ \\
\hline $.13 \%$ & $9.06 \%$ & $.21 \%$ & 550 & $.17 \%$ & 280 & $3.3 \mathrm{~g} / \mathrm{t}$ & $16 \mathrm{~g} / \mathrm{t}$ \\
\hline 180 & 80 & 95 & 100 & 63 & & 24 & $<250$ \\
\hline $.15 \%$ & 470 & $.33 \%$ & 57 & $.32 \%$ & & $<.5 \mathrm{~g} / \mathrm{t}$ & $6.5 \mathrm{~g} / \mathrm{t}$ \\
\hline $23 \%$ & $.20 \%$ & .928 & 20 & 8 & 670 & 39 & $70 g / t$ \\
\hline $.69 \%$ & 90 & 2.237 & 13 & 26 & $.25 \%$ & 150 & $3.5 \mathrm{~g} / \mathrm{t}$ \\
\hline $.60 \%$ & $3.95 \%$ & 360 & $.10 \%$ & 850 & 71 & $<.5 \mathrm{~g} / \mathrm{t}$ & $31.6 \mathrm{~g} / \mathrm{t}$ \\
\hline .117 & 1.797 & 2 & $.38 \%$ & & 2 & $<.5 g / t$ & $20.9 \mathrm{~g} / \mathrm{t}$ \\
\hline 330 & 34 & & 8 & & & $<.5 \mathrm{~g} / \mathrm{t}$ & $<.5 \mathrm{~g} / \mathrm{t}$ \\
\hline $1.14 \pi$ & 530 & 120 & 16 & 18 & & 50 & $45 g / t$ \\
\hline $.24 \%$ & 660 & 80 & 81 & 21 & & 70 & $9.3 \mathrm{~g} / \mathrm{t}$ \\
\hline $5.63 \%$ & 330 & 350 & 140 & $.06 \%$ & & 540 & $40 \mathrm{~g} / \mathrm{t}$ \\
\hline
\end{tabular}

NOTE: Les valeurs pour Ag et Au sont en ppb et celles pour $\mathrm{Cu}, \mathrm{Zn}$, Ni, $\mathrm{Pb}, \mathrm{Cr}$ et Co sont en ppa, a molns d'indications contraires.

TABLEAU 3: Teneurs des minérallsations provenant des principaux 1ndices étudiés 
mafiques au sud-ouest de lac Ham. Knuckey et al. (1982) ont d'autre part reconnu ce dernier type de minéralisation à proximité de lentilles de sulfures massifs volcanogènes stratiformes pour le cas de la mine Millenbach en Abitibi. Bouchard (1986) observe également des minéralisations similaires dans le secteur de la mine Gwillim, au nord-ouest de nos terrains et leur attribue également un âge syn-volcanique.

\subsubsection{Indice du lac Ham (1)}

Au nord-est du lac Ham, un filon-couche gabbroĩque, prenant place dans la Formation de Gilman, renferme des filonets de magnétite, pyrite et chalcopyrite 1 de même que des valeurs erratiques en argent (26 $g / t$ ) et or (figure 8). Ces filonets occupent des plans de cassure en tension, orientés grossièrement NNW $\left(340^{\circ}\right)$ avec un pendage subvertical (planche 33). Ces cassures sont plus ou moins perpendiculaires à la direction de cette masse gabbroĩque. Il $n^{-} \mathrm{y}$ a pas de décrochement apparent de part et d’autre de celles-ci. La situation géologique locale indique que les polarités sont vers le nord: ce filon-couche différencié montre une base à tendance pyroxénitique du coté sud et une partie gabbroĭque riche en phénocristaux de quartz à son sommet, du coté nord.

La superficie fracturée s'étend sur 20 à $25 \mathrm{~m}$ de large par $75 \mathrm{~m}$ de long, à flanc de colline. Les épontes sont fortement silicifiées et chloritisées et ces altérations se propagent même à 1'extérieur de la zone minéralisée, dans les laves mafiques du coté nord de la tranchée 


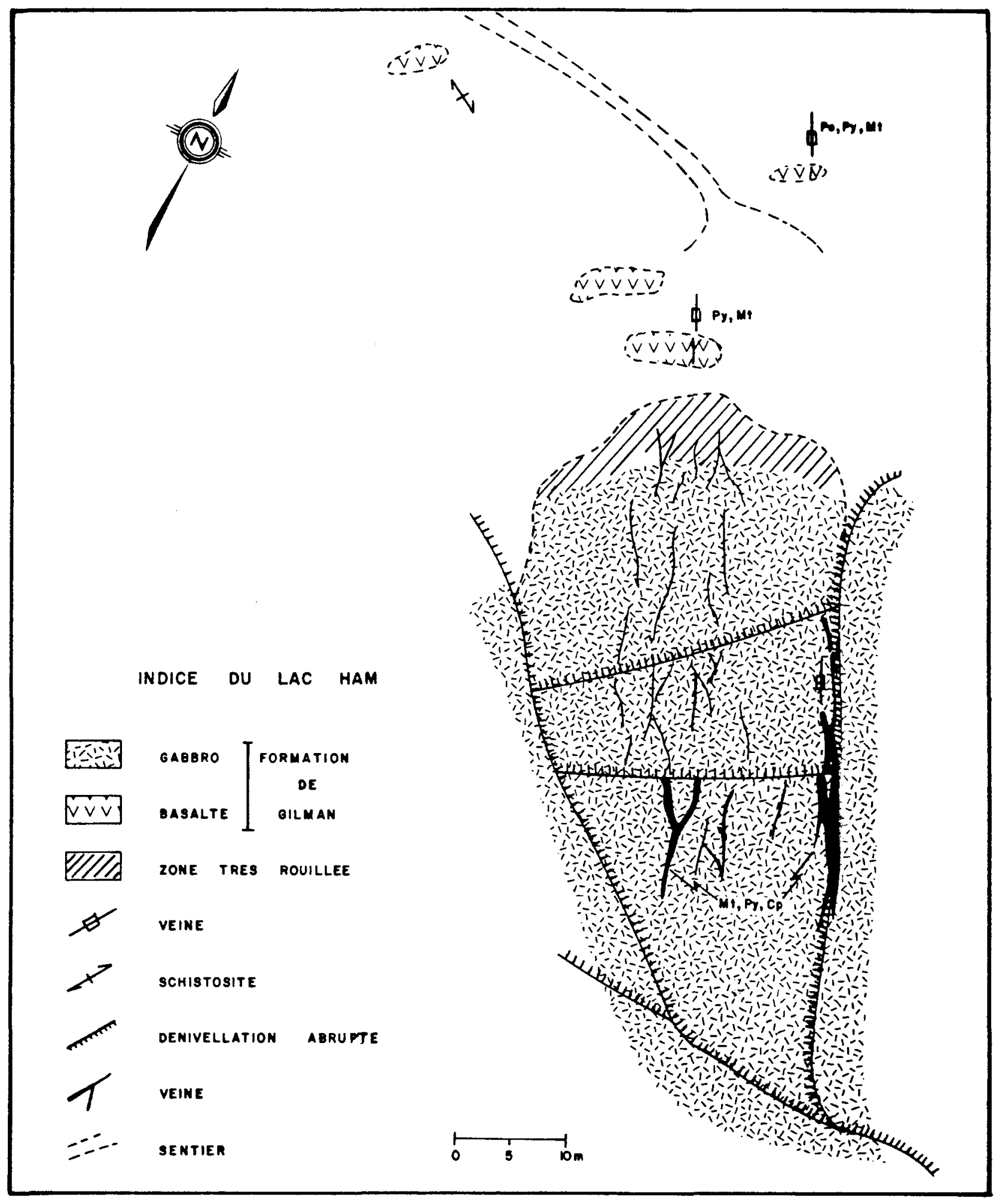

Figure 8. Représentation schématique de 1 'indice minéralisé du lac Ham. Modifiée de Pilote et al. (1984b). 
(planche XVI (A)). Par contre, la carbonatisation et $1^{-e ́ p i d o t i s a t i o n ~ s o n t ~}$ très bien limitées à la zone minéralisée. Celle-ci se compose de veines de magnétite massive, ayant jusqu’à $40 \mathrm{~cm}$ de 1arge. La magnétite est fréquemment bréchifiée et cimentée par I'assemblage pyrite-chalcopyrite, ce qui témoigne de 1'ordre de déposition de 1'oxyde et des sulfures (planche XVI (B)). Toutes ces minéralisations montrent des évidences de recristallisation tardive (planche XVI (C)).

Les meilleures teneurs produites par forages sont de $1.02 \mathrm{~g} / \mathrm{t} \mathrm{Au}$ et $2.05 \% \mathrm{Cu}$ sur 1.7 mètre (Alexander, 1969). L'extension nord de cet indice se perd sous un ruisseau, une faille majeure ENE en occupe possiblement le lit. Par contre, les cassures WNW se poursuivent dans les laves encaissantes. De la tourmaline est localement présente le long de ces fractures. Vers le sud, au sommet de la colline, les cassures disparaissent rapidement ainsi que toute trace de minéralisation.

\subsection{Formation de Blondeau}

$$
\text { De minces lentilles riches en pyrite et pyrrhotine se retrouvent }
$$

très fréquemment dans cette formation. Elles sont conformes au litage. Un bon exemple de ce genre de minéralisation est donné par les sédiments cherteux lités de la Formation de Blondeau, immédiatement sous-jacents aux laves variolaires au sud du lac Larone et au sud du lac Devilliers. Les lits minéralisées ont des puissances de deux à $10 \mathrm{~mm}$. $\mathrm{D}^{-}$autres horizons minéralisés mineurs, de composition surtout pyriteuse, se retrouvent à différents niveaux dans cette formation. Une origine diagénétique plutôt qu'hydrothermale pourrait cependant être 
responsable d'une bonne part de ces derniers types d’horizons minéralisés (Carignan et al., 1984).

5.3.1 Indices des lacs Larone et Devilliers (2) et (3)

Ces deux indices se situent dans des contextes stratigraphiques similaires, soit sous-jacents à un niveau basaltique variolaire. Au sud du lac Larone 2 , la minéralisation se présente sous la forme de minces lits essentiellement pyriteux, stratiformes (planche XVII (A) et (B)), ayant une extension latérale d’au moins 400 mètres. La carbonatisation et $1 a$ chloritisation sont faibles, étroitement restreintes aux lits minéralisés.

Dans le secteur immédiatement au sud du lac Devilliers 3 , une partie de la minéralisation est constituée de minces lentilles stratiformes contenant localement des quantités significatives de chalcopyrite, de sphalérite et de pyrrhotine (figure 9). La pyrite y est d'une façon remarquable très peu abondante, comparée à la pyrrhotine. Ces lentilles ont des épaisseurs diverses, de quelques millimètres à une dizaine de centimètres de large. Elles prennent place au contact entre des volcaniclastites plus ou moins fracturées et une coulée basaltique massive.

A d’autres endroits sous ces niveaux de laves, ces lentilles stratiformes recouvrent des veinules minéralisées discordantes qui bréchifient les volcaniclastites encaissantes (planche XVIII (A)). Ces 


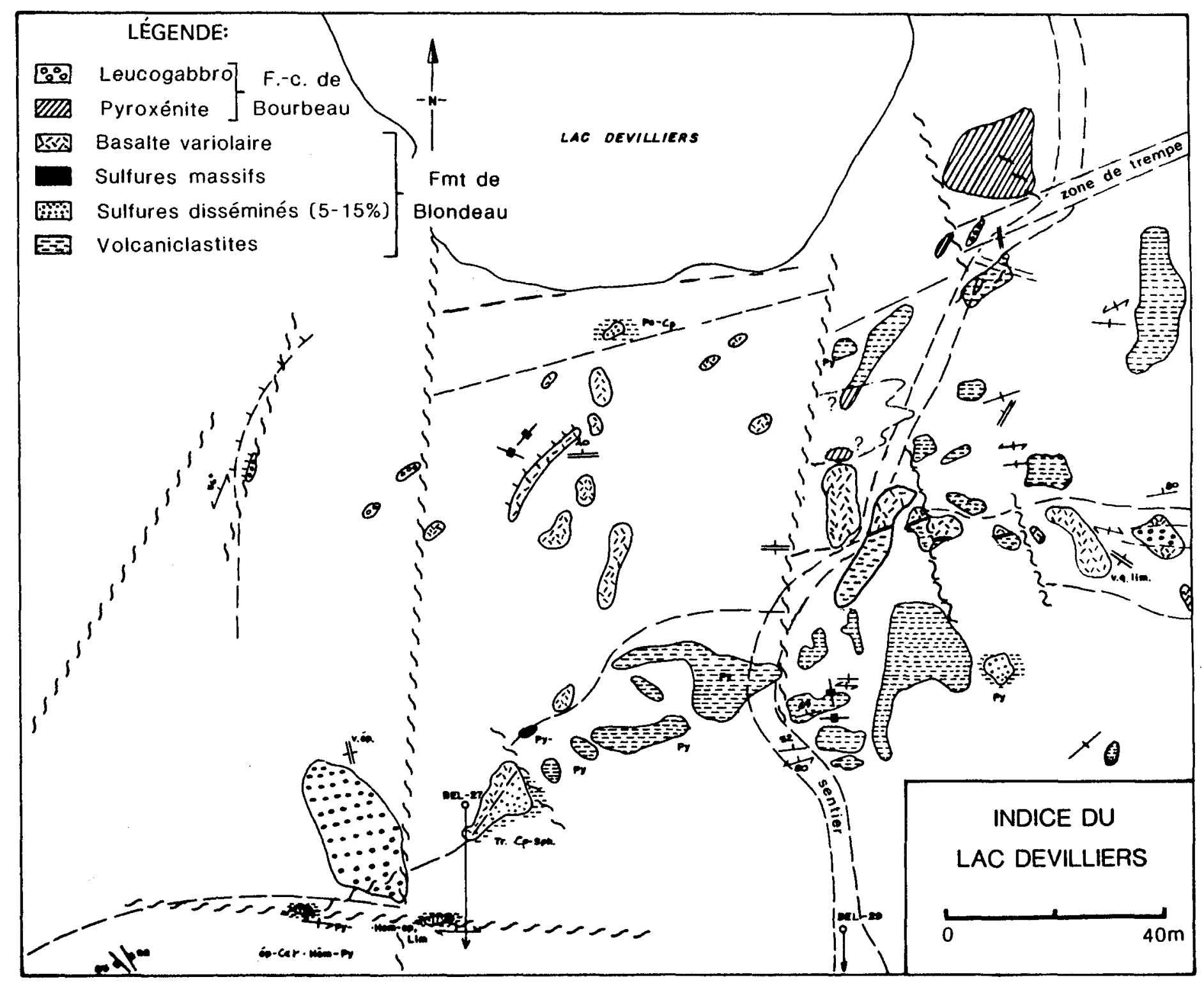

Figure 9. Indice du lac Devilliers, Formation de Blondeau, Mont Bourbeau. Modifiée de Pilote et al. (1984b). 
veinules contiennent de la pyrrhotine et parfois de la pyrite finement disséminées. La chloritisation et dans une moindre mesure la silicification et la carbonatisation affectent toutes les roches-hôtes dans les environs immédiats de ces minéralisations mais ces altérations toutefois ne se propagent pas de façon significative à $1^{\prime}$ intérieur de 1'horizon de lave variolaire.

Les veinules de type discordant sont également étroitement restreintes aux sédiments et tufs sous-jacents à ce niveau de lave. Elles ne pénètrent pas ce niveau. Ce dernier point suggère que l'épanchement des laves est postérieure à la mise en place des minéralisations stratiformes et filoniennes. Ceci est corroboré par le fait que des fragments de blocs minéralisés similaires, contenant de minces lits pyriteux et graphiteux, se retrouvent en inclusions dans ces coulées variolaires (planche XVIII (B)). Ces laves sont surmontées dans ces deux secteurs d'un corps ultramafique (coulée massive, dyke ou filon-couche) de composition pyroxénitique. Dans le cas de $1^{\prime}$ indice du lac Devilliers, la base du filon-couche de Bourbeau n'est qu’à une dizaine de mètres au

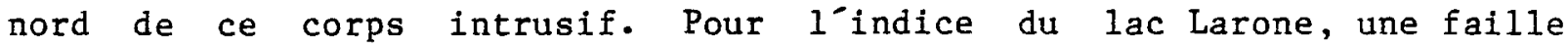
inverse borde du côté nord cette roche pyroxénitique et amène la répétition d'une partie des 1ithologies de la Formation de Blondeau.

Ces lentilles minéralisées sont, au sud-est du lac Devilliers (cf. chap. 3.2.2), sus-jacentes à des coulées de débris régulièrement monolithologiques contituées de blocs, de fragments de composition intermédiaire à felsique ou souvent même pyriteux. Des tufs à 
cristaux, de puissance variable: de 10 à $150 \mathrm{~cm}$, des bombes volcaniques et même localement des lentilles composées de fragments sub-anguleux porphyriques et rhyolitiques sont rencontrés. Ces lithologies recouvrent des horizons de composition mixte, d'une nature davantage silteuse et/ou argileuse marqués par de nombreuses fractures et déformations syn-sédimentaires telles que des glissements et des figures de charge. L'extension latérale de ces séquences volcaniclastiques fragmentaires, typiques d'une activité volcanique felsique proximale, est restreinte au secteur du mont Bourbeau.

Le litage est orienté ENE, avec un pendage variant de 50 à $80^{\circ}$ vers le nord. Ce litage devient par contre nord-est à la périphérie ouest de cette zone. Cette torsion est probablement due à $1^{\text {'effet }}$ d'entrainement sénestre d’une ou de plusieurs failles majeures d'orientation NE.

La chloritisation, la silicification et la carbonatisation représentent vraisemblablement des altérations hydrothermales liées à la venue des minéralisations, dans le secteur du lac Devilliers. Ces altérations affectent de différentes façons les roches encaissantes. Ainsi, à cet endroit, la chloritisation et dans une moindre part la silicification affectent toutes les roches. Par contre, dans la partie ouest, les horizons fragmentaires lités montrent des nucleus altérés de façon préférentielle par la séricite et le carbonate (planche XIX (A)). Ce cortège d’altérations se propage de façon préférentielle dans le sens du litage, dessinant sur $1^{\text {'}}$ affleurant des pseudo-fragments et bandes discontinues (planches XIX (B), XX (A) et (B)). Le métamorphisme de 
contact du pluton du lac Line a de plus affecté ces assemblages et est peut-être en partie responsable des altérations observées.

\subsection{Formation de Blondeau et bordures des filons-couches du Complexe de Cummings}

5.4.1 Indices des Dômes du Radar (4a)

Au nord des dômes du radar, les sédiments volcaniclastiques et tufs de la Formation de Blondeau sont décrits en contact avec la base du filon-couche de Roberge (Gobeil, dans Allard et al., 1979). Néanmoins, une corrélation avec les lithologies présentés du coté ouest (carte en pochette) et un levé magnétique au sol (exécuté par les Mines Camchib Inc.) indiqueraient $q^{\top}{ }^{\top} i l$ pourrait plutôt $\mathbf{s}^{`}$ agir de la base du filon-couche de Bourbeau. Une faille de chevauchement mineur, orientée est-ouest, aurait sa trace hypothétique immédiatement au nord des dykes porphyriques en feldspath et quartz. Cette faille accolerait la pyroxénite de base du filon-couche de Bourbeau sur la pyroxénite noire du filon-couche de Roberge.

Dans la partie centrale de ces indices (figure 10), de minces lits conformes de pyrrhotine et de pyrite de 3 à $12 \mathrm{~mm} \mathrm{~d}$ épaisseur, imprégnées de carbonates, se présentent sur une puissance de $40 \mathrm{~cm}$ à quelques mètres au sud de la base du filon-couche (une zone de trempe de 2 à 3 mètres de large). Un sédiment noirâtre à grains fins, contenant des fragments cherteux, tronque avec un léger angle le long d'une discordance d'érosion la bordure supérieure de ces minéralisations. Le litage va de NE à NNE avec des pendages de $55^{\circ}$ à $80^{\circ}$ vers le NW. De façon générale, les 


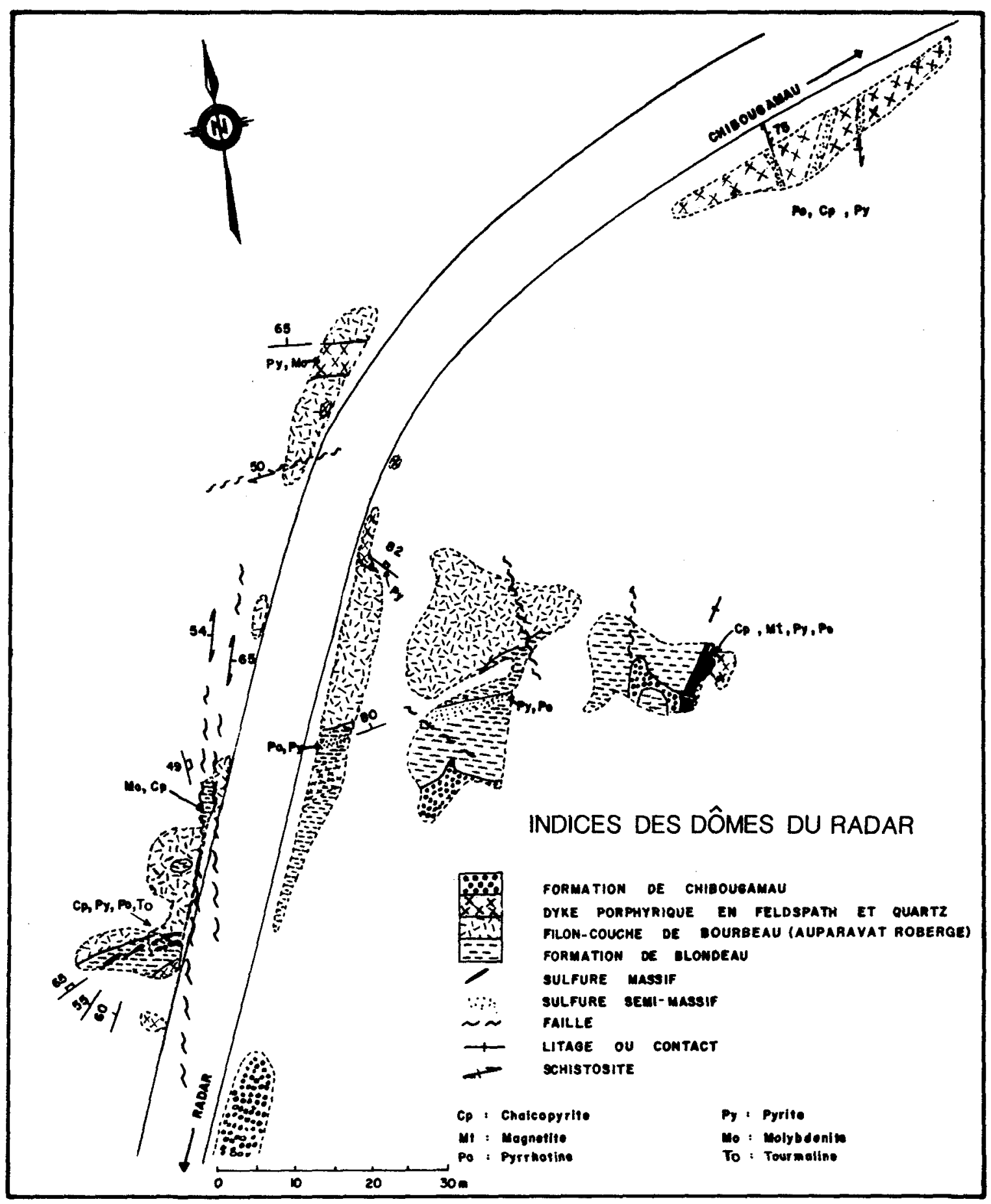

Figure 10. Indices des Dômes du radar, mont Bourbeau. Modifiée de Pilote et al. (1984b) et de Gobeil (dans Allard et al., 1979). 
volcaniclastites sont très silicifiées et fracturées. Du coté ouest de la route, une veine de chalcopyrite massive avec plus ou moins de pyrite et de pyrrhotine est légèrement discordante par rapport au litage. Cette veine fait de 40 à $60 \mathrm{~cm}$ de large. Elle se subdivise en deux segments vers 1'ouest. De la tourmaline $s^{-} y$ trouve disséminée et souligne une foliation parallèle aux contacts (planche XXI (A)).

Du coté est, une épaisse lentille minéralisée se compose de chalcopyrite, de magnétite et plus ou moins de pyrrhotine et de pyrite. Elle est disposée de façon globalement conforme au litage, ce dernier étant orienté NNE et de pendage subvertical. Cependant, cette lentille recoupe en quelques endroits la stratification, ce qui lui confère un caractère filonien. Le sédiment encaissant du coté ouest est très chloritisé. Cette lentille est accolée du coté est à une brèche constituée de fragments cherteux parfois rubannés (figure 10). Cette brèche est cimentée par un matériel tonalitique, porphyrique en plagioclase, similaire en composition aux dykes situés plus au nord (Pilote et al., 1984b). Le conglomérat de la Formation de Chibougamau, d’âge Aphébien (Long, 1974), recouvre en discordance ces diverses lithologies. Une fenêtre au travers du conglomérat permet en plus de voir cette dernière lentille minéralisée et les volcaniclastites encaissantes. Ceci nous donnant la relation d’âge suivante, ce type de minéralisation au moins est pré-Protérozoĩque (planche XXI (B)).

Dans ce secteur, des failles affectent la base présumée du filon-couche de Roberge, les strates de la Formation de Blondeau et les 
dykes porphyriques en feldspath et quartz. Ces failles sont orientées NS et NNE $\left(014^{\circ}\right)$, de pendages variables, à décrochements apparents sénestres et tapissées localement de sidérose. Les dykes porphyriques contiennent par endroits des traces de pyrite, de chalcopyrite et localement de molybdénite le long de ces cassures NS et NE. La molybdénite se retrouve également dans un cisaillement NNE, associée à du quartz et du carbonate (planche (XXI (c)).

5.4.2 L'indice d’argent (4b)

Cet indice prend place dans de minces fractures d'orientation NNW, subverticales (figure 11). Les décrochements apparents sont de très faibles à nuls. Ils affectent la pyroxénite basale du filon-couche de Bourbeau et les volcaniclastites sous-jacentes de la Formation de Blondeau, soit des grès, silts et tufs à fragments felsiques localement porphyriques. Les minéralisations, composées de chalcopyrite, de pyrite et de sphalérite sont associées à des veinules de quartz de 2 à $10 \mathrm{~cm}$ de large, se retrouvant dans ces fractures (planche XXII (A) et (B)). Des teneurs significatives en $\mathrm{Au}$ et $\mathrm{Ag}$ ont été obtenues en surface et en forages. Ces cassures disparaissent rapidement dans la pyroxénite, du coté nord. Les veines de quartz sont cependant le plus large dans la zone de trempe et dans les volcaniclastites immédiatement sousjacentes. Quelques minces fractures minéralisées, discontinues, se retrouvent aussi du coté sud dans la Formation de Blondeau.

La silicification est d'une intensité inégale mais elle affecte 


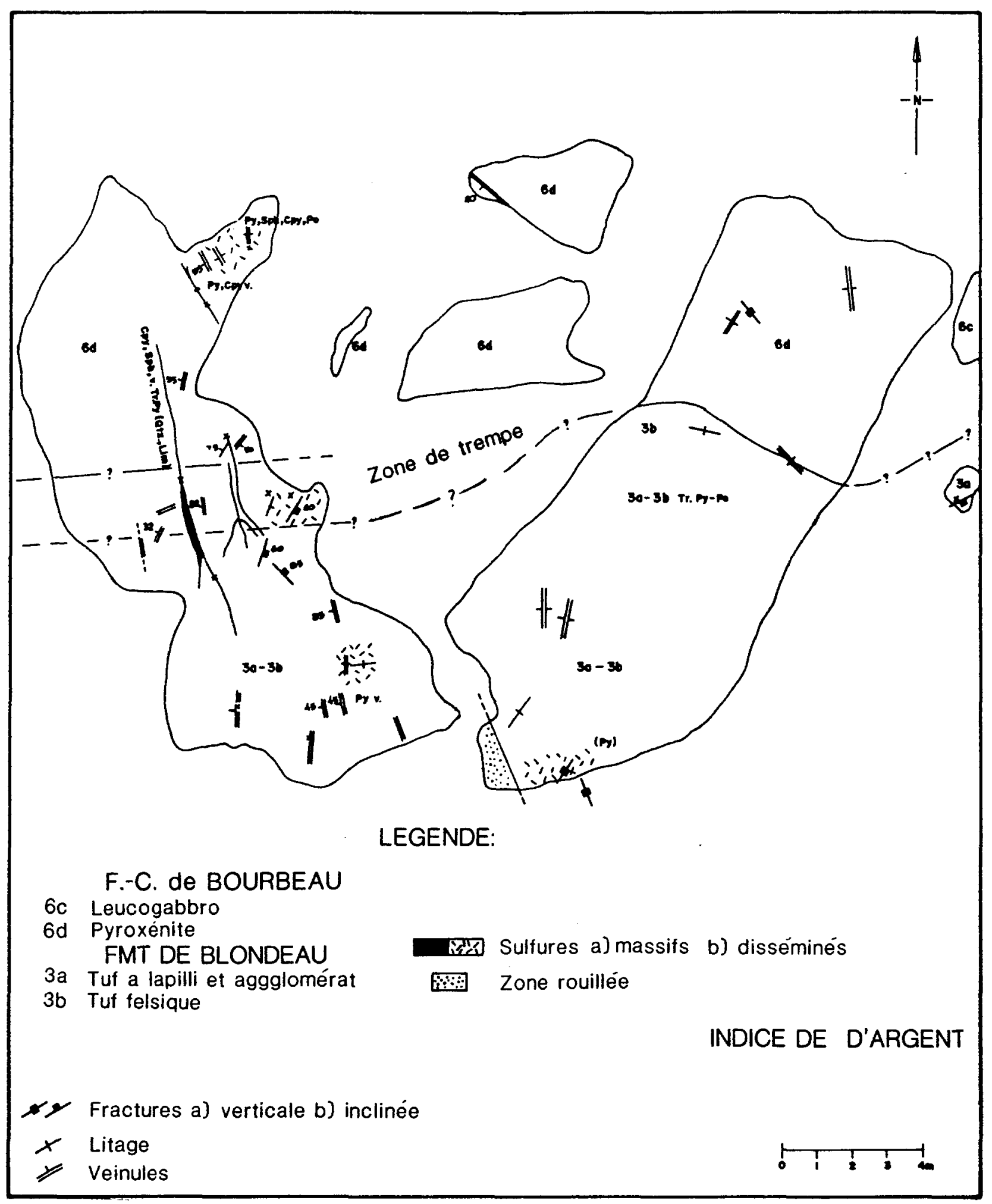

Figure 11. L'indice d'argent, situé à la base du filon-couche de Bourbeau et des volcaniclastites de la Formation de Blondeau, mont Bourbeau. Les termes "tuf à lapilli, tuf felsique et agglomérat" ne représentent que des appellations de terrain. Modifiée de Pilote et al. (1984b). 
toutefois la plus grande partie de cette zone d’affleurements. L'altération autour des fractures minéralisées dessine un patron de pseudo-bréchification dans les épontes (planche XXIII (A)). Un étroit front d'altération chloriteux de 6 à $20 \mathrm{~cm}$ de large enserre de chaque coté les fractures (planches XXIII (B)) tandis que la séricitisation se fait sentir de façon préférentielle à l'extérieur de cette zone jusqu’à une distance de un ou deux mètres au maximum (planche XXIV (A)). La carbonatisation est en général faible et associée étroitement aux veinules minéralisées. A une distance supérieure à trois mètres, les altérations sont minimales et permettent de déterminer la nature des lithologies présentes, des coulées pyroclastiques composées de fragments rhyolitiques similaires à ceux rencontrés dans les environs du lac Devilliers (planche XXIV (B)).

5.4.3 Apophyse du filon-couche de Bourbeau (5)

Cette zone d’affleurements, située à 300 mètres à $1^{\prime}$ est du lac Devilliers, présente trois indices minéralisés distincts (figure 12). A cet endroit une apophyse péridotitique, liêe au filon-couche de Bourbeau, s'introduit dans les sédiments et tufs à fragments de la Formation de Blondeau. Les strates sont de direction $E-W$ et les pendages sont de subverticaux à abrupts vers le nord.

Un premier indice minéralisé occupe une zone de cisaillement de direction NNW à pendages subverticaux à abrupts vers 1 'est. I1 consiste en d'étroites veinules de chalcopyrite, sphalérite et pyrite, de 2 à 12 


\section{CONTACT FILON-COUCHE DE BOURBEAU ET FORMATION BLONDEAU, FLANC NORD DU MONT-BOURBEAU}

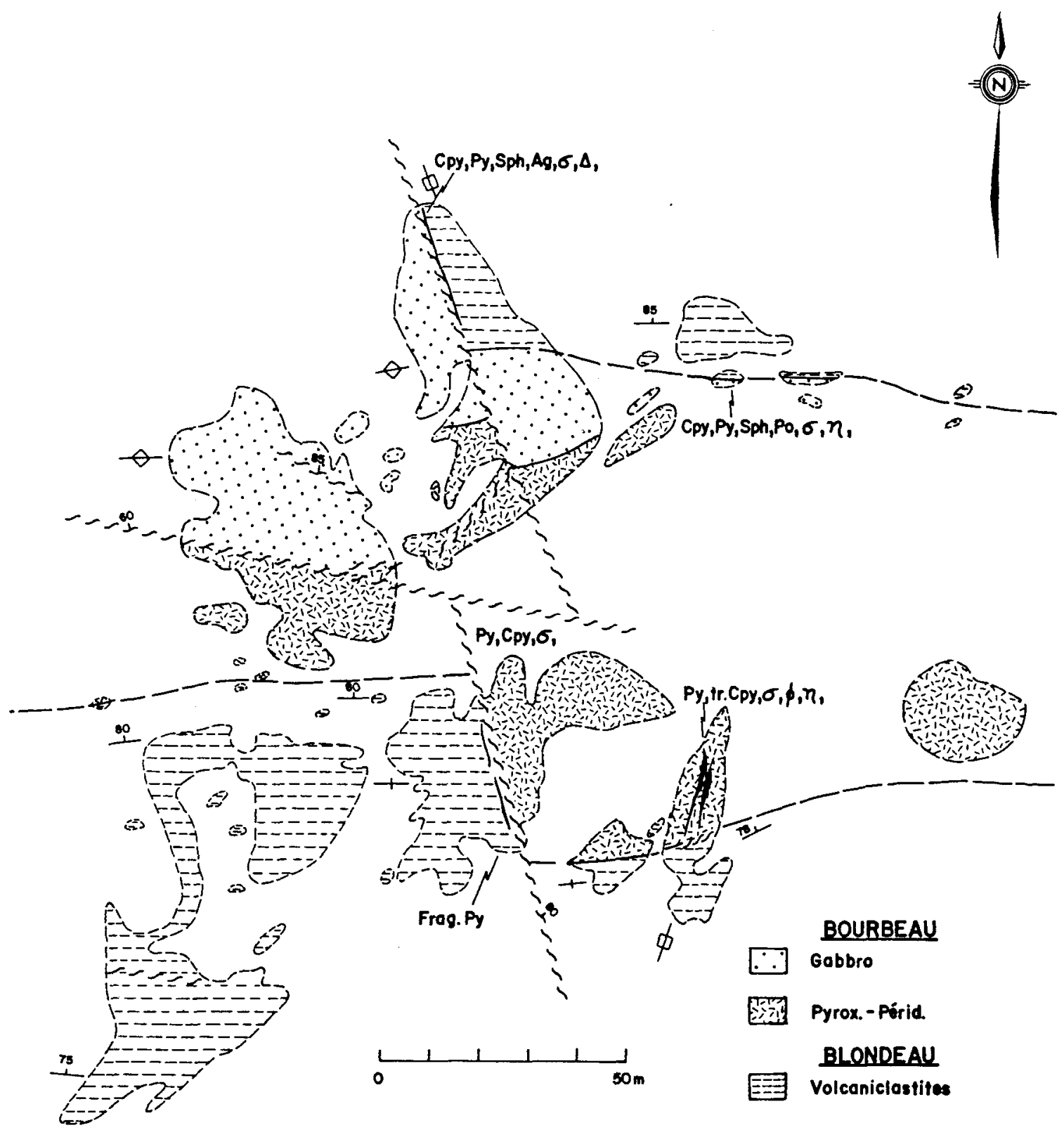

Figure 12. Schéma des indices minéralisés se retrouvant dans les environs d'une apophyse de composition dunitique du filon-couche de Bourbeau, injectée dans les volcaniclastites de la Formation de Blondeau, mont Bourbeau. 
$\mathrm{mm}$ de large. Les valeurs en $\mathrm{Ag} \mathrm{y}$ sont erratiques, le résultat d`une analyse indiquant entre autre plus de $100 \mathrm{~g} / \mathrm{t}$ dans un échantillon pris à 1 'intérieur de ce cisaillement, dans la partie nord de cette zone. La minéralisation tend à se retrouver dans des fractures parallèles, du côté de la Formation de Blondeau et non directement dans le cisaillement principal NNW qui sépare ces roches du filon-couche de Bourbeau (planche XXV). Les épontes sont fortement chloritisées et silicifiées. A d’autres endroits, ces fractures ne portent aucune minéralisation mais la chloritisation et la séricitisation dessinent sur $1^{-}$affleurement des structures pseudo-fragmentaires, tout à fait

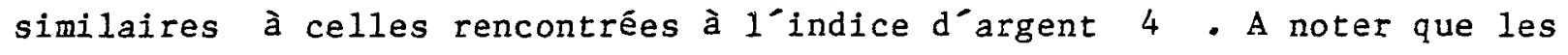
cisaillements majeurs NNW montrent un léger décrochement dextre, d`au plus une quarantaine de mètres d’amplitude.

Un second indice est représenté par des filons d'orientation NNE $\left(014^{\circ}\right)$, subverticaux et $d^{\prime}$ une nature essentiellement pyriteuse. Ceux-ci $n^{\prime}$ ont indiqué après analyse aucune valeur appréciable en or et en argent. Ces filons sont composés de pyrite granuleuse (en cubes de 2 à $3 \mathrm{~mm}$ d'arête) s'effritant très facilement au toucher. La largeur de ces filons va de 5 à $15 \mathrm{~cm}$. Ils se retrouvent dans la base du filon-couche de Bourbeau mais les altérations, une forte silicification et de plus faibles et restreintes chloritisations et carbonatisations, affectent également les roches sousjacentes de la Formation de Blondeau. Les

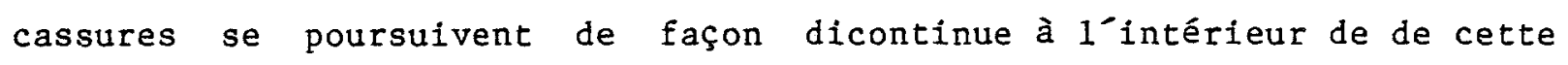
formation mais elles sont alors stériles. 
Le troisième indice minéralisé est situé dans un cisaillement $\mathrm{E}-\mathrm{W}$, marquant le contact entre un leucogabbro et des volcaniclastites. Le cisaillement est d’une intensité variable, généralement faible et d’une largeur inférieure à une trentaine de centimètres. Il comprend de minces filonets, de 2 à $6 \mathrm{~mm}$ de large, de pyrrhotine, de chalcopyrite, de sphalérite et de pyrite. Des valeurs significatives en Ag sont présentes (59 $\mathrm{g} / \mathrm{t})$. La carbonatisation est très forte dans le cisaillement même, de faibles silicifications et chloritisations ne se signalant qu’à proximité immédiate de cet indice et ne dépasse pas deux mètres en extension.

5.4.4 Sommet du filon-couche de Ventures 6

A environ 400 mètres au nord-ouest de la Belle Baie, des amas minéralisés substratiformes riches en pyrrhotine se retrouvent dans la Formation de Blondeau, à quelques mètres du sommet du filon-couche de Ventures (planche XXVI (A) et (B)). Ces amas, de 2 à $10 \mathrm{~cm}$ de large, orientés E-W, contiennent également de la pyrite et plus rarement de la chalcopyrite et de la sphalérite. Les altérations sont faibles, la carbonatisation dominant sur la chloritisation. Des filons contenant de la pyrite et de la pyrrhotine aboutissent parfois du filon-couche de Ventures jusqu’à ces amas, sans les traverser de manière significative. Les relations sont toutefois masquées fréquemment par des cisaillements d'intensités variables, subparallèles au contact supérieur de ce filon-couche. 
5.5 Le Complexe de Cummings

5.5.1 Le "Main Showing" (7)

Cet indice est situé immédiatement au nord de la Belle Baie, dans le filon-couche de Roberge (figure 13). La minéralisation est de type veine et se compose de pyrrhotine massive, de pyrite, de chalcopyrite et de sphalérite avec localement de la magnétite. Des valeurs significatives en $\mathrm{Au}$ et $\mathrm{Ag}$ sont localement signalées dans des échantillons recueillis en surface et dans des sondages. Ces minéralisations se présentent sous forme de quatre lentilles de sulfures massifs de .3 à 1 mètre de largeur par de 2 à 8 mètres de longueur. L'extension totale de ces quatre lentilles atteint 60 mètres en surface. Elles sont encaissées dans des cisaillements NS à NNW à pendages subverticaux qui montrent une configuration "pinch and swel1", chaque lentille étant isolée des autres de façon très nette dans cette structure. Les ouvertures le long de la trace de ce cisaillement semblent coincider avec les endroits où cette trace recoupe des zones de composition pyroxénitique. Ces lentilles sont très déformées et semblent contemporaines au cisaillement de direction NS qui les contient.

Les roches encaissantes sont des pyroxénites, péridotites et dunites, localement très bien litées. Ceci pourrait indiquer que cet indice se trouve dans le membre inférieur du filon-couche de Roberge, $d^{\top}$ après la coupe-type qứa établie Poitras (1984) pour celui-ci et confirmée par notre cartographie dans la région au sud du mont Bourbeau. 


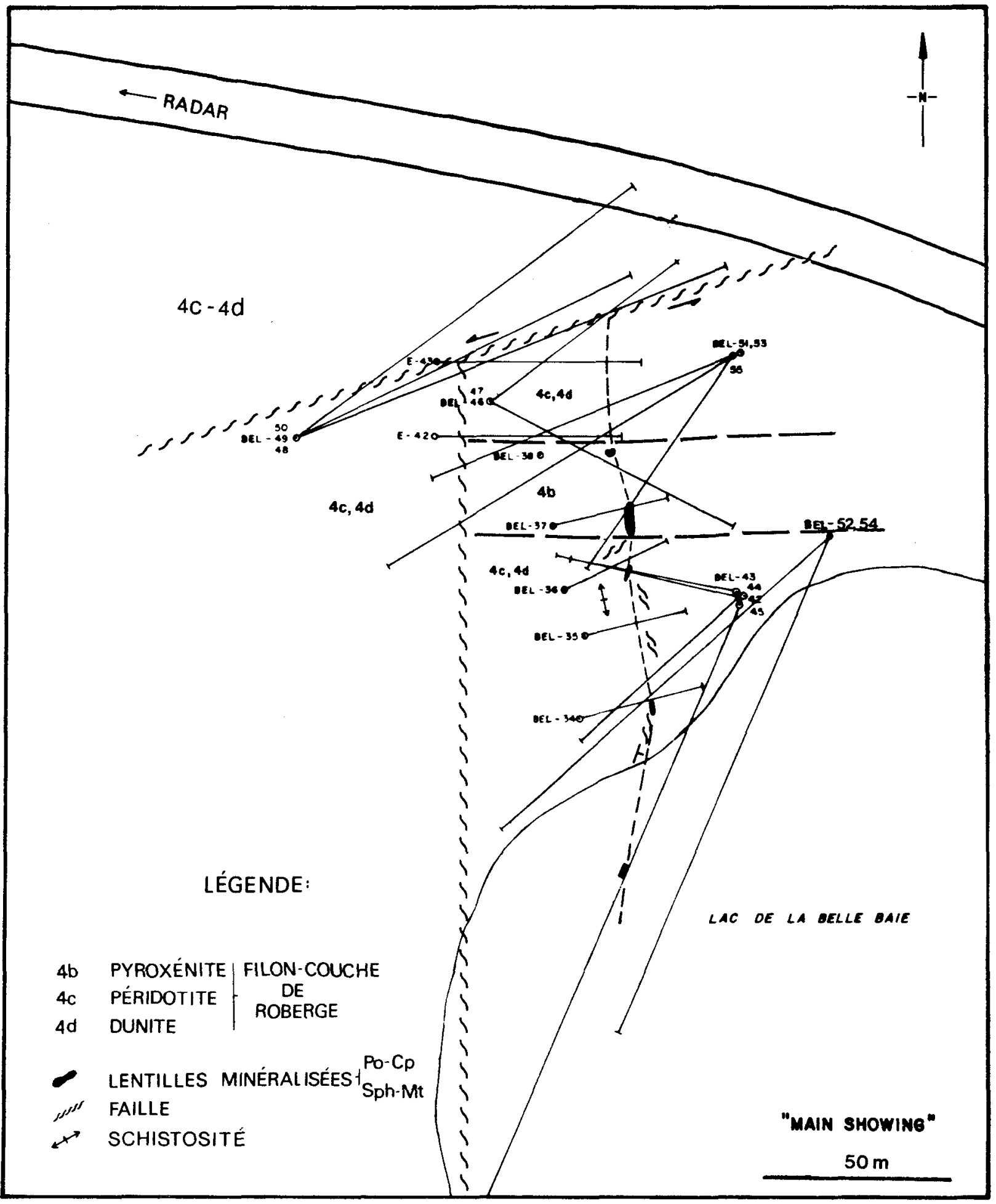

Figure 13. Le "Main Showing", filon-couche de Roberge, mont Bourbeau. Modifiée de Racine (1984). 
Ces lentilles présentent régulièrement la zonation suivante: 1a chalcopyrite et la sphalérite se retrouvant plutôt en bordure et la pyrrhotine davantage vers le centre. Des filonets de chalcopyrite et de sphalérite se retrouvent aussi dans les épontes. La carbonatisation et la chloritisation sont d'intensités variables et généralement restreintes aux environs immédiats des veines minéralisées.

De récents forages (1983-84) effectués par les Mines Camchib Inc. $n^{\prime}$ ont pas permis d'établir du coté nord la poursuite de ces minéralisations. Par contre, ils ont démontré la présence d'un important cisaillement d'orientation E-W qui pourrait être la trace d'une faille inverse majeure, la faille du lac Cummings. Celle-ci pourrait éventuellement expliquer pourquoi ces minéralisations n’ont pas été retrouvées du coté nord.

Au sud du mont Bourbeau, une lentille de composition comparable à celle du "Main Showing" se présente dans un cisaillement NW $\left(320^{\circ}\right)$ avec un pendage de $69^{\circ}$ vers le NE. Cette lentille varie en largeur de 30 à 45 cm par moins de $T$ mètres en longueur. Elle est encaissée dans une péridotite noirâtre, à 1'intérieur du membre inférieur du filon-couche de Roberge. La carbonatisation et la chloritisation sont peu marquées. Des valeurs intéressantes en argent, or et zinc y ont été obtenues. Un cisaillement majeur, d’âge protérozoĩque, passe à quelques dizaines de mètres au nord de cette lentille.

5.5.2 Filons pyriteux, filon-couche de Ventures (8) 


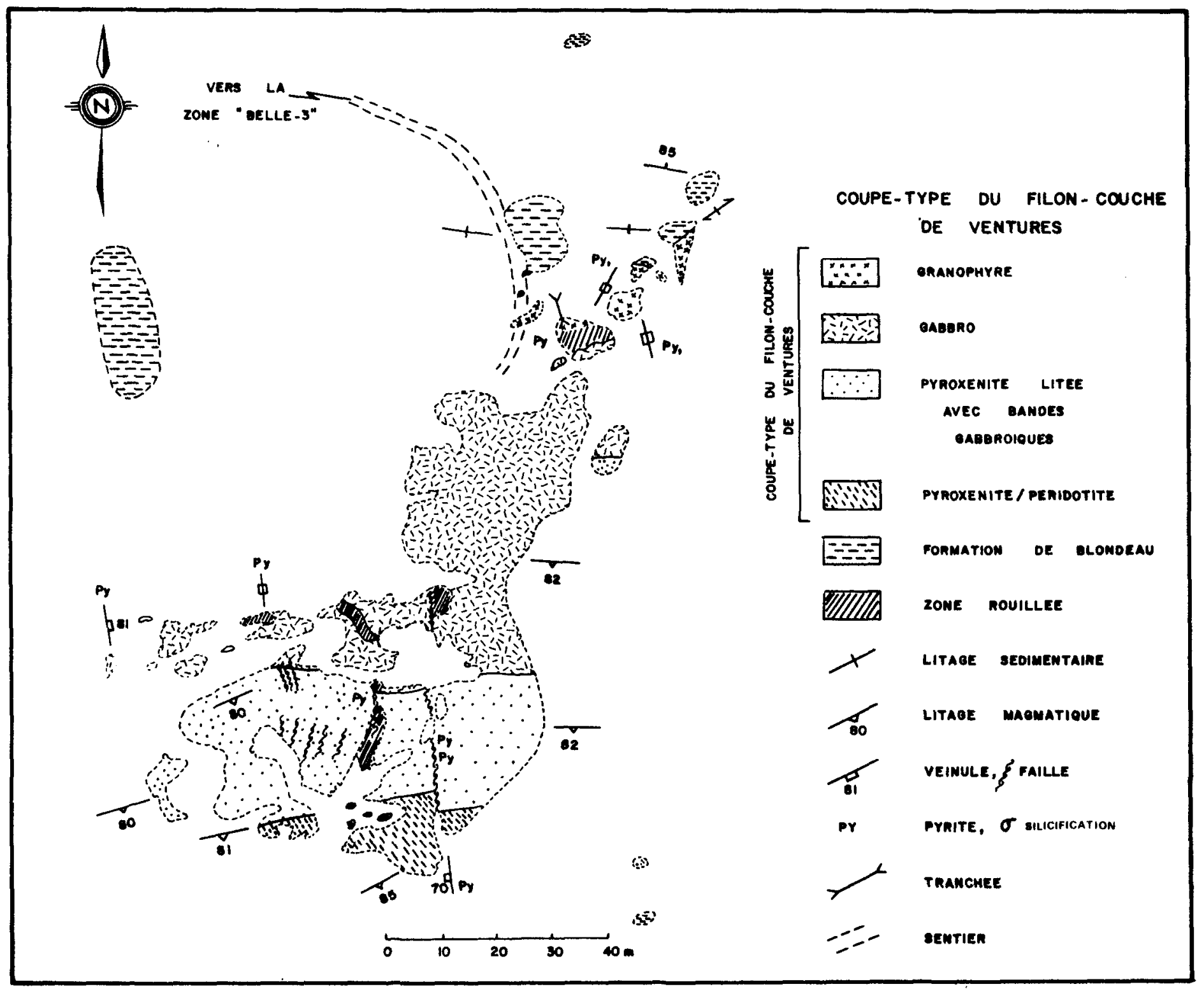

Figure 14. Coupe-type du filon-couche de Ventures et positions de filonets pyriteux, mont Bourbeau. Modifiée de Pilote et al. (1984b). 
Ces indices prennent place à environ 700 mètres au SE du lac Devilliers. Il $s^{\prime}$ agit de filons pyriteux, orientés $N-S$ à NNE et à

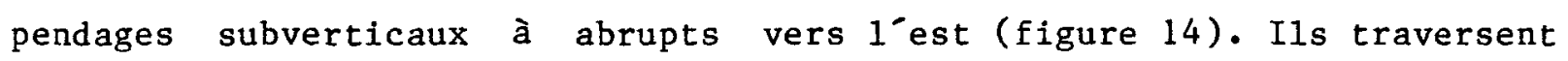
quasi totalement le filon-couche de Ventures pour finalement disparaitre à proximité des volcaniclastites et tufs de la Formation de Blondeau, où leurs extensions ne peuvent être retracées. Les décrochements sont faibles ou non-appréciables de part et d'autre des filons. Ceux-ci ont de quelques millimètres à plus d'une cinquantaine de centimètres de large. La silicification et la carbonatisation sont les altérations les plus importantes mais elles demeurent étroitement restreintes aux bordures des filons minéralisés. A d’autres endroits, ces fractures sont minéralisées en pyrrhotine. Les orientations restent globalement les mêmes, N-S à NNE. Ces filons sont similaires à ceux rencontrés dans des cassures NNE affectant la base du filon-couche de Bourbeau et décrits précédemment.

Certains minces filons pyriteux empruntent également la bordure de dykes gabbroíques introduisant et bréchifiant le membre pyroxénitique inférieur. Ces dykes, de 50 à $70 \mathrm{~cm}$ d’épaisseur, sont également de direction NNE.

\section{5 .3 La zone "Be1-3" 9}

Cet indice doit son nom au numéro du premier forage qui $1^{-}$a intercepté en 1979 (figure 15). Ces travaux, entrepris par les Mines 


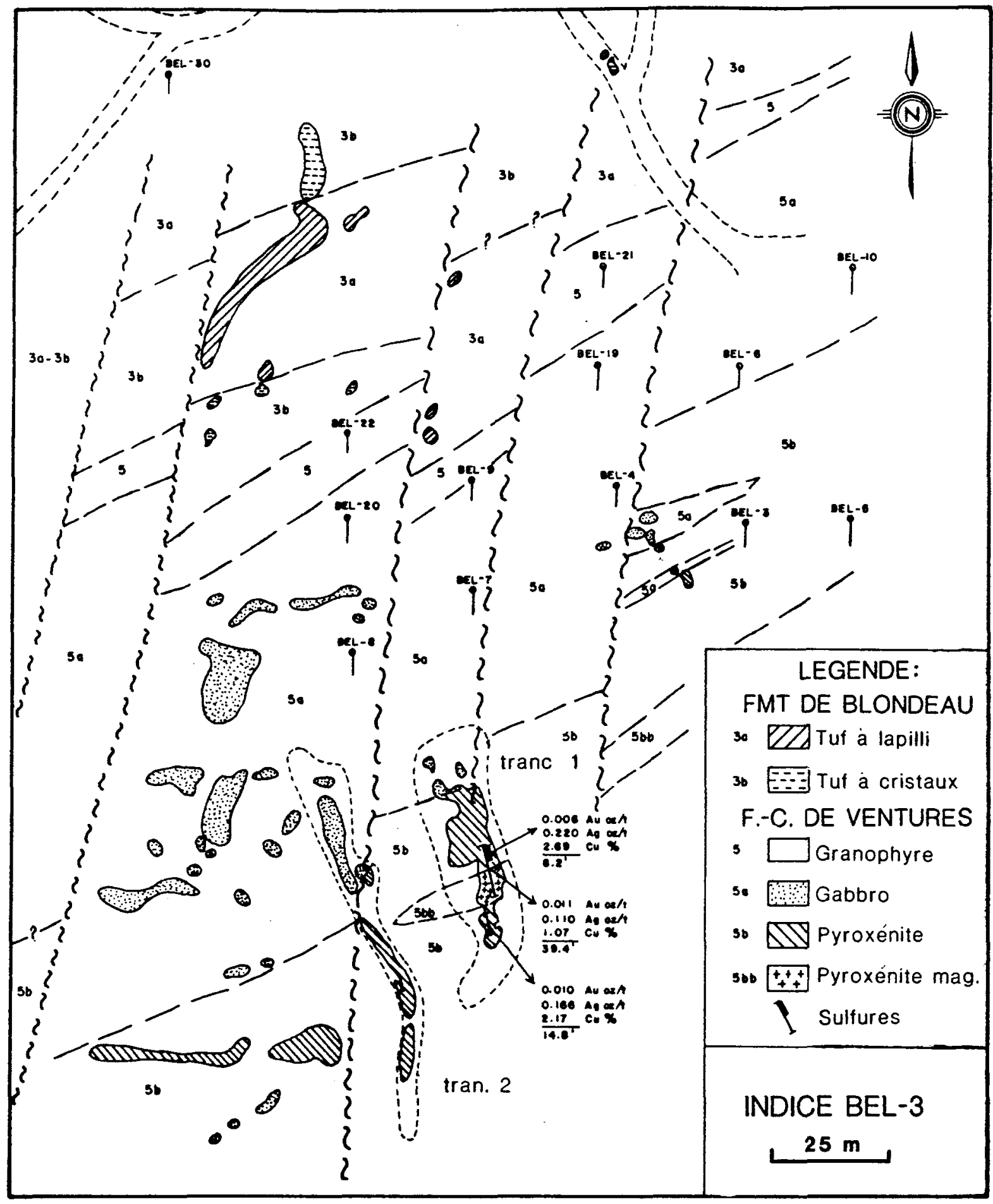

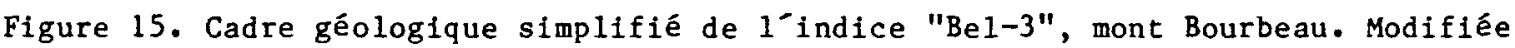
de Pilote et al. (1984b). 
Camchib Inc., faisaient suite à une campagne de prospection géophysique exécutée sur le mont Bourbeau.

Les minéralisations prennent la forme de veines et de lentilles de sulfures semi-massives à massives, globalement conformes en surface. Elles sont composées de pyrrhotine, de pentlandite, de chalcopyrite et de plus ou moins de sphalérite, de magnétite, de quartz et de carbonate. Des valeurs localement importantes en $\mathrm{Ni}$, $\mathrm{Co}$ et $\mathrm{Ag}$ sont rencontrées. La molybdénite a été identifiée sur certaines sections polies mais non décelée à $1^{`}$ analyse chimique. Ces veinules se retrouvent dans les membres pyroxénitiques à gabbroĩques du filon-couche de Ventures. Ceux-ci ont une direction générale ENE $\left(235^{\circ}\right.$ ) avec un pendage de $60^{\circ}$ vers le nord. En surface, ces veines et lentilles sont de faible puissance, de 5 à $15 \mathrm{~cm}$ de large par quelques mètres de longueur. Par contre, une interprétation tirée de différents forages et d'une galerie d'exploration indique que ces veines, subparallèles entre elles, peuvent atteindre jusqu’à 3 mètres de largeur et près de 140 mètres de longueur. Elles demeurent également grossièrement conformes au litage magmatique observé (figures 16 et 17).

Ces veinules sont encaissées dans de légers cisaillements. Les minéralisations sont très déformées, rubannées et plissotées (planche XXVII (A) et (B)). Elles sont de plus recristallisées: des plages de pyrrhotine avec bordures en point triple et exhibant une texture granoblastique sont couramment observées (planche XXVII $(\mathrm{C})$ ). Les textures "decussate" sont également fréquentes. Une zonation est parfois discernable dans ces lentilles. Une mince bande de carbonate (calcite) 


\section{INDICE BEL-3 \\ SECTION SIMPLIFIÉE}

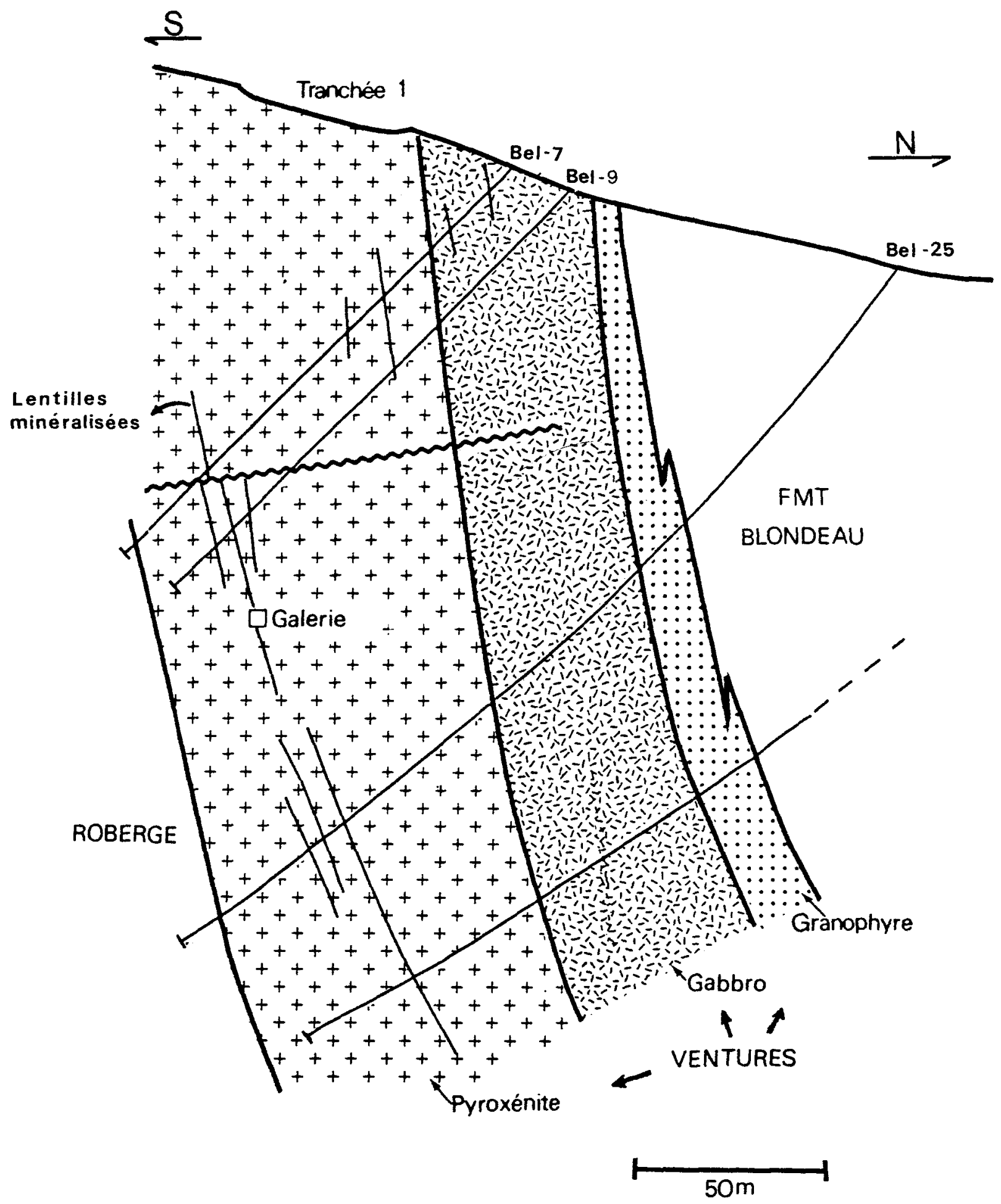

Figure 16. Section simplifiée de 1'indice "Be1-3", mont Bourbeau. Interprétation tirée de trous de sondage et de travaux souterrains. 


\section{GALERIE D’EXPLORATION, ZONE BEL-3}

\section{PLAN SIMPLIFIE}

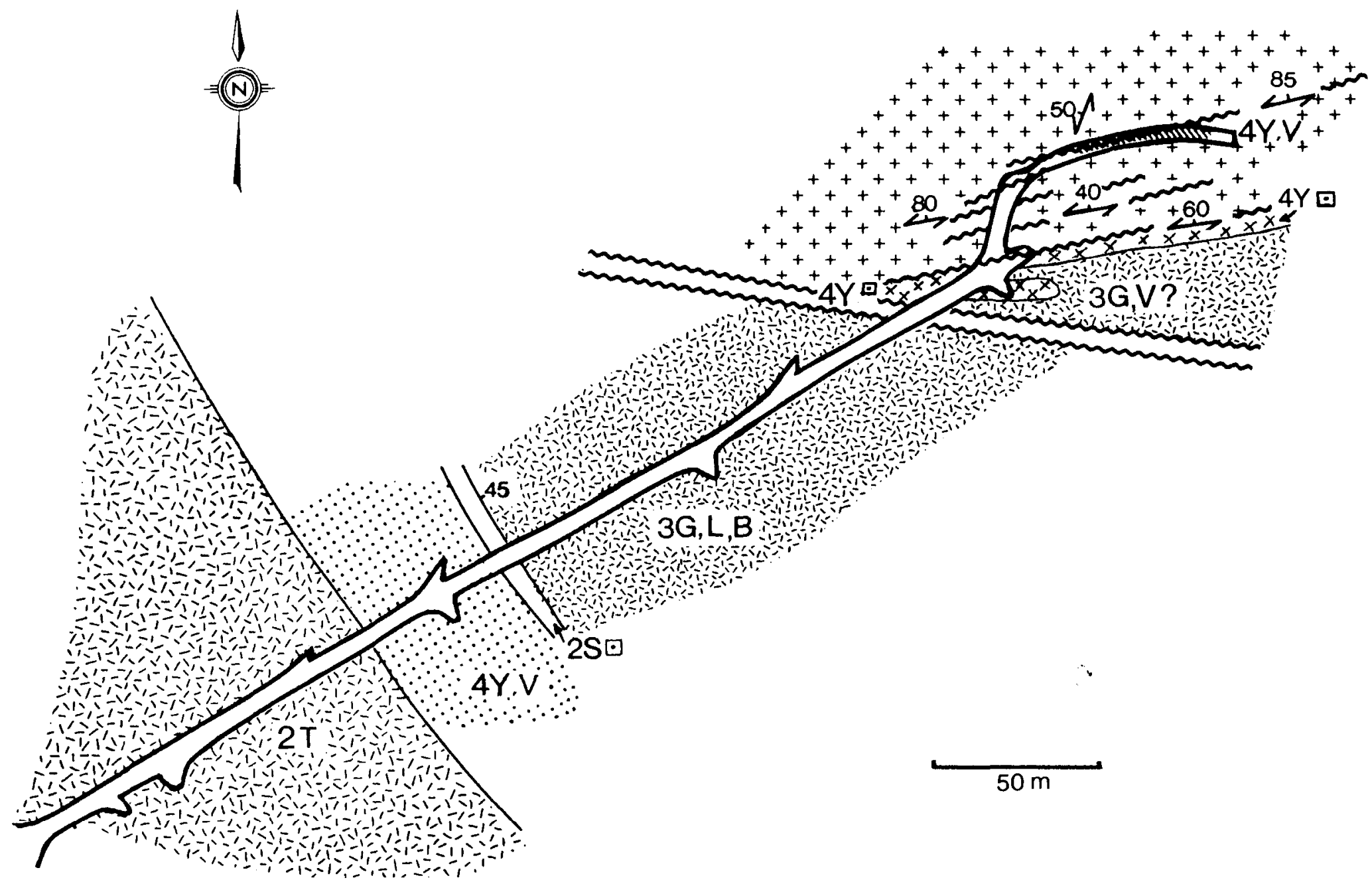

Figure 17. Plan simplifié de la galerie d'exploration "Be1-3", mont Bourbeau. 4YG= pyroxénite poecilitique du filon-couche de Roberge: $4 \mathrm{Y}, \mathrm{V}=$ pyroxénite verte du filon-couche de Ventures; $3 G, V=$ gabbro du filon-couche de Ventures; $3 G, L, B=$ leucogabbro du filon-couche de Bourbeau; $2 \mathrm{~T}$ = tonalt te; $2 \mathrm{SQ}$ = syénite porphyrique; $I I I=$ lentilles minéralisées. Modifiée de Camchib Inc. (rapport interne). 
de quelques centimètres de large borde ces lentilles et les sépare d`une

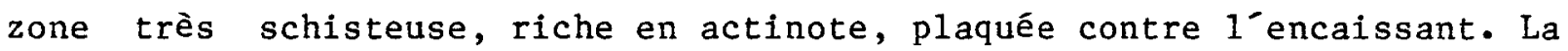
partie externe de ces lentilles est intensément foliée et contient de nombreux xénolithes silicatés très chloriteux et des fragments d'épontes. La partie centrale de ces lentilles est foliée également, mais d’une manière moins intense. Des veinules de chalcopyrite et plus ou moins pyrrhotine bréchifient parfois ces lentilles et se retrouvent même dans les roches pyroxénitiques encaissantes.

Des veinules d’axinite de quelques millimètres d’épaisseur, orientées ENE avec un pendage faible $\left(20^{\circ}\right)$ vers le $\mathrm{NW}$, recoupent sur la tranchée no 1 les roches gabbroĩques. Sur 1 a tranchée no 2 une veine pegmatitique de composition tonalitique, probablement rattachée au stock intrusif du lac Line, s'injecte dans la pyroxénite du filon-couche de Ventures.

Les différents forages (figure 16) et les travaux d'exploration sousterrains figure 17) ont permis d'interpréter $1^{\prime}$ existence de grands cisaillements d'orientation NE, à décrochements apparents régulièrement sénestres. Les pendages sont inclinés de $50^{\circ}$ vers $1^{\text {'ouest. Ces }}$ cisaillements décalent les lentilles minéralisées et expliquent probablement pourquoi celles-ci ne se retrouvent pas sur 1a tranchée no 2 (figure 15). Ces minéralisations ne se poursuivent pas de manière

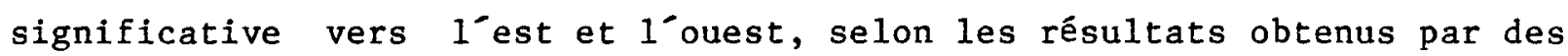
campagnes de forage. Par contre le décalage possible, sénestre, amené par les failles de direction NE est à considérer dans la reconstruction de 


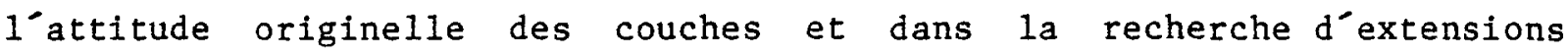
possibles.

Ces différents travaux d'exploration soulignent également le fait que le filon-couche de Roberge se retrouve seulement à quelques dizaines de mètres au sud de ces lentilles. La galerie d'exploration montre, avant d’atteindre ces minéralisations, une pyroxénite noire poecilitique (figure 17), typique du filon-couche de Roberge (Poitras, 1984; Pilote 1983 et 1984). La zone de contact entre les filons-couches de Ventures et de Roberge $y$ est alors marquée par une alternance de pyroxénite noire magnétique et de pyroxénite verte, les lentilles minéralisées se retrouvant le plus souvent dans cette dernière lithologie. Au sud de la pyroxénite noire poecilitique, se présente une masse gabbroĩque typique du filon-couche de Ventures. Cette masse $s^{-}$appuie du coté sud sur une faille de chevauchement majeur de direction $E-W$, la faille du lac Cummings. Ce gabbro peut avoir cet emplacement expliqué par un mouvement inverse mineur, subsidiaire au mouvement majeur qui $s^{-}$est fait sur la faille prịncipale plus au sud ou encore il peut s’agir d’une injection gabbroìque dans la partie supérieure du filon-couche de Roberge.

Au sud-ouest du lac Line, de minces filons pyriteux contiennent parfois de la chalcopyrite et des valeurs erratiques en Ag et Au. Ces filons font rarement plus de 1 centimètre de large par moins de 1 mètre de long. Ils prennent place également dans le filon-couche de Ventures. Le caractère filonien des minéralisations est cependant très net ici. La carbonatisation est forte et étroitement restreinte aux zones 
minéralisées

5.6 Stock intrusif du lac Line

(10)

(11)

Des indices minéralisés en chalcopyrite, sphalérite, pyrite et molybdénite (planche XXVIII (A)) se retrouvent à la périphérie est de cet intrusif au sud de la Baie du Cran Penché, dans des veines discontinues de quartz. Celles-ci sont orientées NE-SW, avec des pendages sud abrupts. Ces veines lenticulaires font rarement plus de $20 \mathrm{~cm}$ de large par $3 \mathrm{~m}$ de long. Elles se présentent dans le stock tonalitique ou encore dans les péridotites du filon-couche de Roberge. La silicification est étroitement restreinte aux bordures des veines et une séricitisation et une altération potassique diffuse affectent plus globalement cette zone.

Dans les environs de ces affleurements, de nombreux petits dykes tonalitiques s'injectent également dans les faciès ultramafiques du filon-couche de Roberge. La tonalite y montre localement dans ses plans de fracture de la chalcopyrite en trace associée à du carbonate.

Au nord-ouest du lac Ham, la tonalite est localement bréchifiée le long de courts plans grossièrement rectilignes, d'extension inférieure à 5 mètres. La puissance de ces "corridors" bréchifiés va de 5 à $20 \mathrm{~cm}$ (planche XXVIII (B)). Un carbonate de fer cimente les fragments plutoniques. Il $\mathrm{n}^{-} \mathrm{y}$ a pas de minéralisations significatives, sauf localement des traces de pyrite.

Sur 1a rive est $d^{\top}$ un petit $1 \mathrm{ac}$, à 600 mètres à $1^{-}$ouest du $1 \mathrm{ac}$ Ham, 
la tonalite est fracturée en plusieurs endroits le long de plans Ns à localement NE, à pendage abrupt vers 1'ouest. Quelques fractures sont minéralisées en chalcopyrite et en argent $(45 \mathrm{~g} / \mathrm{t})$. Elles font rarement plus de 2 ou $3 \mathrm{~m}$ de long, par 1 ou $2 \mathrm{~cm}$ de large. L'encaissant tonalitique est carbonatisé et chloritisé. Des laves de la Formation de Gilman sont situées à proximité. Cet encaissant contient localement des veinules d’axinite. Aucune trace significative en molybdène n’a été décelée ici par notre échantillonnage.

\subsection{Protérozoĩque et Formation de Chibougamau}

Nous n’avons observé aucune minéralisation significative encaissée dans ces dépôt prétérozolqques lors de nos campagnes de terrains. Par contre ceci $n^{-}$implique aucunement $q u^{-} i 1 n^{-} y$ ait pas d'autres types de minéralisations, contemporaines ou postérieures à ces dépôts, encaissées dans ceux-ci ou encore dans les roches supracrustales archéennes ailleurs dans le district (Chown, 1984; Guha, 1984; Thorpe et a1., 1984).

Ainsi sur le mont Bourbeau, un filonet minéralisé en chalcopyrite, pyrite et galène, encaissé dans la Formation de Blondeau et recoupé par un trou de sondage, s'est vu assigné un âge protérozoĩque d’après sa composition isotopique en plomb (Thorpe et a1., 1984). Au sud du lac

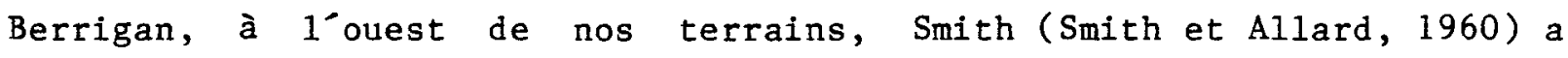
d’autre part observé dans les dépôts protérozoĩques quelques petites veinules de pyrrhotine, de chalcopyrite et de sphalérite. Dans le secteur du lac Waconichi, différentes minéralisations filoniennes recoupent les 
dépôts protérozoĩques, soit entre autres les indices Portage, Blondeau et Bouzan. 
CHAPITRE 6

DISCUSSION

\subsection{Introduction}

La disposition sur le terrain des différents types d’indices décrits précédemment, de configurations stratiformes et filoniennes, suggère que des contrôles tant stratigraphiques que structuraux ont fortement dirigé leurs mises en place. Nous discuterons de ces contrôles pour chacun de ces types.

6.2 Minéralisations volcanogènes et leur mise en place

La majorité des minéralisations stratiformes ainsi qu'un bon nombre de nature filonienne se classent dans cette catégorie. Ces indices stratiformes sont régulièrement conformes au litage, quand ce dernier est discernable. Ils comprennent les minces lits minéralisés en pyrite observés dans la Formation de Gilman. A I'intérieur de la Formation de Blondeau, ces minéralisations comprennent $1^{-}$indice du lac Devilliers et les amas sub-stratiformes composés de pyrrhotine et pyrite situés à la base du filon-couche de Bourbeau et au sommet du filon-couche de Ventures.

La plupart de ces indices se retrouvent sur le mont Bourbeau. A cet endroit et particulièrement dans le secteur du lac Devilliers, les différents faciès volcaniclastiques de la Formation de Blondeau

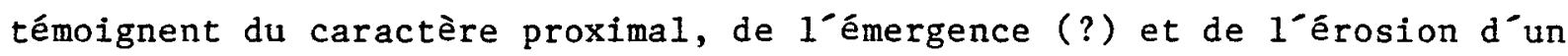


centre volcanique felsique. Dans cet environnement, même des séquences silteuses et argileuses montrent couramment leurs structures syn-sédimentaires (décrochements mineurs et glissements) comblées partiellement par des carbonates et des sulfures, le plus souvent de la pyrrhotine et de la pyrite, provenant possiblement des centres exhalatifs alors opérant.

Un des centres exhalatifs reconnus sur ce terrain semble être situé également dans les environs immédiats du lac Devilliers (figure 9). Outre le fait d’y observer des minéralisations stratiformes en chalcopyrite, sphalérite et pyrrhotine, de type proximal (Large, 1977; Plimer, 1978) quand comparées à celles observées au sud du lac Larone, cet endroit se signale par une association caractéristique d’altérations: une silicification, une carbonatisation et une chloritisation poussée (tableau 4). En affleurement, des horizons grèseux montrent des pseudo-sphérulites intensément silicifiées et carbonatisées, enrobées de quartz microcristallin (planche $X X(A)$ et (B)). La matrice de cette roche est intensément chloritisée. Gibson et Watkinson (1979) ont identifié des processus de silicification préférentiels, liés à 1'existence d'une porosité suffisante ou de fractures, qui peuvent affecter sélectivement certaines lithologies suivant les conditions environnantes.

Suite à ces observations, un certain ordre chronologique peut être établi dans la séquence d'événements survenus dans le secteur du lac Devilliers. Tout $d^{`}$ abord une forte silicification semble avoir affectée 


\begin{tabular}{|c|c|c|c|c|}
\hline $\begin{array}{l}\text { Formations ou } \\
\text { localisations }\end{array}$ & Composition & Configuration & Altération & Orientation \\
\hline $\begin{array}{l}\text { G1l man, } \\
\text { lac Ham (1) }\end{array}$ & $\begin{array}{l}-M t-C p y-P y+A g \\
\quad \pm A u\end{array}$ & $\begin{array}{l}\text {-fflonienne, dans un } \\
\text { fllon-couche gabbroique }\end{array}$ & $\begin{array}{l}\text {-silicification et carbonatisation } \\
\text { fortes, séricitisation falbie }\end{array}$ & -NNW, subvertical \\
\hline $\begin{array}{l}\text { Blondeau, } \\
\text { au sud du lac } \\
\text { Larone (2) }\end{array}$ & $-\mathrm{Py}-\mathrm{Po}_{0}$ & $\begin{array}{l}\text {-lentille, sous un hori- } \\
\text { zon de basalte variolaire; }\end{array}$ & -faible carbonatisation & -stratiforme \\
\hline lac Devilliers (3) & $\begin{array}{l}- \text { Po-Cpy-Sph-Py } \\
+\mathrm{Ag}\end{array}$ & $\begin{array}{l}\text {-même configuration mais } \\
\text { en plus présence a proxi- } \\
\text { mité de fragments } \\
\text { pyriteux; des filons miné- } \\
\text { ralisés bréchifient les } \\
\text { volcaniclastites susjacentes }\end{array}$ & $\begin{array}{l}\text {-silfcification et carbonatisation } \\
\text { fortes sulvies ultérleurement de } \\
\text { silfcification et de chloriti- } \\
\text { sation intenses }\end{array}$ & $\begin{array}{l}\text {-stratiforme et } \\
\text { discordante }\end{array}$ \\
\hline $\begin{array}{l}\text { Dômes du } \\
\text { Radar ( } 4 a)\end{array}$ & $-\mathrm{CPy}_{\mathrm{P}}-\mathrm{Mt}-\mathrm{Po}_{\mathrm{O}}-\mathrm{Py}$ & -lentille filonfenne & -silicification et carbonatisation & -sub-stratiforme \\
\hline $\begin{array}{l}\text { Indice d'argent } \\
\text { ( } 4 \mathrm{~b}) \text { et apophyse } \\
\text { du f.-c. de } \\
\text { Bourbeau ( } 5 \text { ) }\end{array}$ & $\begin{array}{c}\text { Cpy-Sph-Py } \\
\pm A g \pm A u\end{array}$ & -filonienne & $\begin{array}{l}\text {-sllicification suivi de } \\
\text { séricitisation et de chloriti- } \\
\text { sation intenses }\end{array}$ & $\begin{array}{l}\text {-NNW, pendage } \\
\text { subvertical à } \\
\text { abrupt vers } \\
\text { le SW }\end{array}$ \\
\hline $\begin{array}{l}\text { A la base du } \\
\text { f. }-c \text {. de Bourbeau } \\
\text { et au sommet du } \\
\text { f.-c. de Ventures } \\
(6)\end{array}$ & - $\mathrm{Po}_{\mathrm{O}}-\mathrm{Py}_{ \pm}+\mathrm{Cpy}_{ \pm} \mathrm{Sph}$ & -minces lits discontinus & $\begin{array}{l}\text {-faible chloritisation des épontes } \\
\text { et carbonatisation forte en pré- } \\
\text { sence de cibaillement }\end{array}$ & -stratiforme \\
\hline $\begin{array}{l}\text { Complexe de Cumaings } \\
\text { base du } f .-c \text {. de } \\
\text { Bourbeau ( } 5 \text { ) }\end{array}$ & - Py & -filonienne & -silicification & -NNE, subvertical \\
\hline $\begin{array}{l}\text { le Main Showing } \\
\text { ( } 7) \text { et l Indice } \\
\text { au sud du mont } \\
\text { Bourbeau }\end{array}$ & $\begin{array}{c}-P o-C p y-S p h-P y \\
+M t+A g+A u\end{array}$ & -filonienne & -carbonatisation & $\begin{array}{l}\text {-NS à NNW, } \\
\text { subvertical }\end{array}$ \\
\hline $\begin{array}{l}\text { le prospect } \\
\text { Taché }\end{array}$ & $\begin{array}{r}-S_{p h}-\mathrm{Po}-\mathrm{Gn}-\mathrm{As} \\
-\mathrm{C}_{\mathrm{py}}-\mathrm{Py} \pm \mathrm{Ag} \pm \mathrm{Au}\end{array}$ & -filonienne & -silicification et carbonatisation & $\begin{array}{l}-010^{\circ} a 030^{\circ}, \text { pendage } \\
\text { vertical et vers } 1^{\prime} \text { est }\end{array}$ \\
\hline $\begin{array}{l}f \cdot-c \text {. de } \\
\text { Ventures ( } 8)\end{array}$ & $-P y \pm P o$ & -filonienne & -silicification et carbonatisation & $\begin{array}{l}\text {-NS à } 015^{\circ} \\
\text { subvertical }\end{array}$ \\
\hline Indice Bel-3 & $\begin{array}{l}-C_{p y-P d-P o-M t} \\
- \text { Sph+Mo et } \\
\text { valeurs en } \mathrm{Ni} \\
\text { Co et } \mathrm{Ag}\end{array}$ & $\begin{array}{l}\text {-lentilles aux bordures } \\
\text { cisalllées }\end{array}$ & $\begin{array}{l}\text {-légères carbonatisation, chlort- } \\
\text { tisation et silicification }\end{array}$ & $\begin{array}{l}\text {-NE, subvertical à } \\
\text { abrupt vers le nord }\end{array}$ \\
\hline $\begin{array}{l}\text { minéralisation } \\
\text { d'origine magwa- } \\
\text { tique? a l'ouest } \\
\text { du site ( } 8)\end{array}$ & $\begin{array}{l}\text { - Po et valeurs } \\
\text { en } \mathrm{Ni} \text { et } \mathrm{Cr}\end{array}$ & -filonienne & -silicification & -NNE, subvertical \\
\hline $\begin{array}{l}\text { dykes de PFQ à la } \\
\text { base du f.-c. de } \\
\text { Bourbeau (?) (4a) }\end{array}$ & $-\mathrm{Py}-\mathrm{Cpy} \pm \mathrm{Po} \pm \mathrm{MO}$ & -filonienne & $\begin{array}{l}\text {-faibles carbonatisation et } \\
\text { silicification }\end{array}$ & $\begin{array}{l}\text {-NNE, pendage abrupt } \\
\text { et de sens variable }\end{array}$ \\
\hline $\begin{array}{l}\text { Stock tonaliclque } \\
\text { au sud de la Baie } \\
\text { du Cran Penché(10) }\end{array}$ & $-C_{p y}-\mathrm{Sph}-\mathrm{Py} \pm \mathrm{Mo}$ & -veines de quartz & $\begin{array}{l}\text {-silfctfication et carbonatisation } \\
\text { faibles }\end{array}$ & $\begin{array}{l}\text {-NE, pendage nord } \\
\text { abrupt }\end{array}$ \\
\hline $\begin{array}{l}\text { à l'ouest du lac } \\
\text { Ham (11) }\end{array}$ & - Cpy \pm Ag & -filonienne & -carbonatisation et chloritisation & $\begin{array}{l}\text {-NNW a NE, pendage } \\
\text { vers } 1 \text { 'ouest }\end{array}$ \\
\hline
\end{tabular}

TABLEAU 4. Sommaire des différents indices minéralisés et de leurs caractéristiques. 


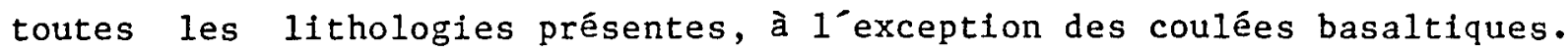
Ceci suggére que ces coulées ne s’étaient alors pas encore épanchées. Par 1a suite aurait pris place une forte carbonatisation, toujours accompagnée de la silicification. Ceci donnant comme résultat des noyaux, consistant en des cristaux ou fragments, où la silicification s'est amorcée et a été suivie d'une carbonatisation diffuse. La silicification s’est poursuivie et a enrobé ces pseudo-sphérules. Certains de ces sphérules, selon l'intensité des processus d’altération, se sont agglutinés en bandes compactes blanchâtres allongées préférentiellement selon la stratigraphie (planche XIX (A) et (B)). Par 1a suite une chloritisation intense est venue recouper ces bandes et s'est aussi propagée préférentiellement selon le litage.

Si on voulait se déplacer en direction d'un (ou des ?) éventuel centre volcanique felsique, dont les flancs seraient bordés par ces minéralisations stratiformes, il faudrait se diriger soit vers $1^{\prime}$ 'est, vers la mine aurifère Norbeau ou soit vers le nord, vers le lac Bourbeau. Malheureusement, la trace axiale du synclinal. de Chibougamau est interprétée comme passant à ce dernier endroit et nous empêche d'observer plus en détail les lithologies susjacentes (Duquette (1970, 1976 et 1982). L'extension des différents faciès de 1a Formation de Blondeau vers $1^{\prime}$ ouest ne représente sur ces terrains qu’un milieu bassinal distal où ne prend place qưune sédimentation de fond marin interrompue que par quelques façiès volcanogènes (Archer, 1983, 1984).

$D^{`}$ autres évidences nous suggèrent également une faible distance, de 
1 -ordre kilomètrique au maximum, entre le secteur du mont Bourbeau et ce centre volcanique felsique. Ces évidences sont, premièrement, la présence de fragments pyriteux, exclusifs au secteur du lac Devilliers (mont Bourbeau) et se trouvant dispersés dans un mince horizon volcaniclastique à 1'intérieur de la Formation de Blondeau et, deuxièmement, la fréquence accrue de failles syn-volcaniques qui affectent également les trois filons-couches du Complexe de Cummings. Ces failles, d'orientation nord-nord-est à nord-sud, sont rencontrées plus particulièrement dans le secteur du lac Devilliers et près des indices des dômes du radar. Des failles d'orientation similaire encaissent et bordent $1^{-}$indice de $\mathrm{Zn}-\mathrm{Pb}$ du prospect Taché (lac Berrigan) et affectent les filons-couches de Roberge, de Ventures ainsi que la Formation de Blondeau (Pilote, 1986).

De façon plus particulière, le dépôt de zinc, plomb, or et argent du prospect Taché (Pilote et al., 1984a; Pilote 1986), situé dans le prolongement ouest de nos travaux de cartographie, est une évidence indirecte qu'un épisode de fracturation a pris place durant la période d’activité volcanique. Ce dépôt est en effet d`âge Archéen ( 2.72

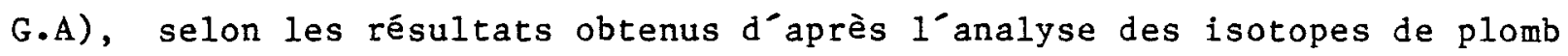
contenus dans ces minéralisations (Thorpe et al., 1981 et 1984). Cet âge est globalement comparable à d’autres estimations tirées de dépôts de sulfures massifs stratiformes d’âge volcanique observés ailleurs dans le camp minier de Chibougamau. Les structures minéralisêes y sont orientées de $010^{\circ}$ à $030^{\circ}$, avec des pendages subverticaux à abrupts vers 1'ouest. Le fait que la roche-hôte soit représentée par les membres ultramafiques du filon-couche de Roberge et localement du 
filon-couche de Ventures $n^{\prime}$ indique en fait que ceux-ci possèdent un comportement mécanique propice à la fracturation.

Sur le mont Bourbeau, des cassures similaires, de direction $\mathrm{N}-\mathrm{S}$ à $030^{\circ}$ avec pendage abrupt, sont très fréquentes. Elles affectent autant les différents façiès de la Formation de Blondeau que les trois filons-couches du Complexe de Cummings. Elles sont également d’échelles mineures et ne causent pas de décalage important dans les lithologies encaissantes. Les indices stratiformes et filoniens (décrits précédemment) de la Formation de Blondeau et les indices filoniens à la base du filon-couche de Roberge (le "Main Showing", quoique de direction NNW et $1^{-}$indice au SW de la Belle Baie) répondent à ces caractéristiques. Ces indices montrent des compositions riches en chalcopyrite, magnétite, pyrrhotine, sphalérite et pyrite.

\footnotetext{
$D^{\circ}$ autres minéralisations, composées surtout de pyrite et de pyrrhotine, remplissent des fractures d'orientation similaire, nord-nord-est, à la base du filon-couche de Bourbeau et au sommet du filon-couche de Ventures, sur le mont Bourbeau. Ce fait, ainsi que le type d'altération qui affecte les épontes de ces filons, à savoir une forte carbonatisation, une silicification et une chloritisation variable, laisse présumer que ces dernières minéralisations sont elles aussi d'origine volcanique. Des travaux sur les isotopes de soufre et d'oxygène pourraient sans doute apporter de nouveaux éléments sur ce point. Pour le moment, nous établissons donc que ce système de courtes cassures orientées $\mathrm{N}-\mathrm{S}$ à $030^{\circ}$ est d’âge Archéen et syn-volcanique (Pilote et
} 
a1., 1984a; Pilote, 1986).

D’autre part, le fait que ce système de cassure réputé d’âge syn-volcanique recoupe les différents filons-couches étagés du Complexe de Cummings implique qu’à cette époque, ce complexe s'était déjà mis en place, à la base de la Formation de Blondeau et qu’il n’aurait pas

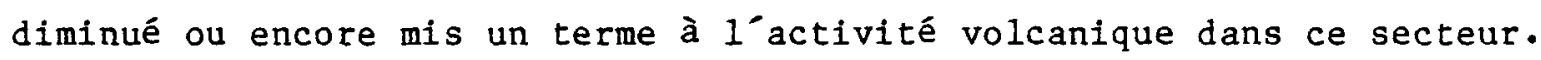

En considération de ces derniers points, nous pouvons supposer que 1a mise en place du Complexe de Cummings, un événement d`âge syn-volcanique, s'est faite à un niveau relativement haut dans le bassin sédimentaire, dans des roches non totalement consolidées et encore riches en eau, et ce à quelques kilomètres seulement de la surface. Ceci a permis aux dykes de filiation volcanique de traverser le complexe et aux fractures syn-volcaniques de chenaliser les systèmes hydrothermaux développés. Une telle mise en place près de la surface est aussi avancée par Poitras (1984) et également observée par Bradley (1965) et Francis (1982) dans le cas d'intrusions doléritiques majeures en Angleterre. Bradley (1965) a de plus suggéré, suite à ses observations, que ces filons-couches s'introduisent généralement durant ou peu de temps après la déposition des sédiments encaissants. Et ceci est vraisembablement ce qui s'est produit aussi dans la région de Chibougamau, puisque les travaux de Cimon et Gobeil (1976) et de Dimroth et al. (1985a) ont permis la reconnaissance de cailloux représentatifs des différents filons-couches du Complexe de Cummings à 1'intérieur de la Formation de Stella, d’âge archéen. 
Un autre aspect à considérer demeure que bon nombre de minéralisations stratiformes de 1 a Formation de Blondeau bordent fréquemment la base du filon-couche de Bourbeau et beaucoup moins fréquemment celle du filon-couche de Roberge. Ces minéralisations se présentent plus rarement au sommet du filon-couche de Ventures. Cette situation implique qu'une bonne partie de ces indices devaient exister auparavant, pour avoir guider de façon préférentielle la mise en place régionale et locale de ces filons-couches (Duquette,1976 et 1982; Allard et Gobeil, 1984; Bélanger et al., 1984; Guha, 1984). Les possibilités $\mathrm{qu}^{\prime}$ il $\mathrm{y}$ ait eu une contribution par remobilisation et enrichissement des sulfures à ces sites ponctuels, dans un pourcentage non-quantifiable, lors d'un événement dynamo-thermique régional ne sont pas discutées dans les travaux précédents et sont à envisager, quoique difficiles à soutenir sans analyses plus poussées.

Ainsi sur le mont Bourbeau, dans le secteur de $1^{-}$apophyse dunitique du filon-couche de Bourbeau (figure 12), des minéralisations filoniennes en pyrrhotine, associées à de fortes valeurs en $\mathrm{Ag}$, sont encaissées dans un léger cisaillement est-ouest, parallèle à la bordure du filon-couche. Ce type de cassure suggère à tout le moins que ces minéralisations sont de pré à syn-tectoniques et pourraient être dues à une remobilisation métamorphique ou encore à la mise en place même du Complexe de Cummings.

En considération de cette dernière idée, Einsele et al. (1980) 
rapportent, d'après leurs études au sud-ouest de la Californie, que 1'introduction d'un filon-couche suffisamment épais amène une forte diminution de la porosité des sédiments encaissants situés sous et sur cet intrusif. L'effet immédiat est de réduire localement 1 'épaisseur de la colonne de sédiments considérés, par expulsion de l'eau intersticielle, sans pour autant affecter la topographie à la surface. Cette réduction $s^{-}$avère être du même ordre de grandeur que l'épaisseur du filon-couche impliqué (Einsele et al., 1980). L'eau expulsée, de haute température, peut rejoindre la surface ou encore si elle est confinée par une quelconque barrière, peut se déplacer latéralement jusqu’à la rencontre d'une structure favorable, une faille et atteindre alors la surface. Cette eau (ou ce fluide ?) peut aussi s'accumuler en un certain point et générer la création d’un aquifère, si la pression de confinement le permet.

Les filons-couches du Complexe de Cummings ont fort probablement pu agir comme d’excellentes barrières au passage des fluides hydrothermaux volcanogènes, dès leur consolidation. Ils représentent de plus une source de chaleur appréciable pour faciliter la remobilisation potentielle des indices déjà présents à ce moment dans la Formation de BIondeau, dans diverses cassures d`orientations particulières. Ceci pourrait expliquer partiellement la présence de quelques amas plus ou moins conformes qui se retrouvent dans des structures cassantes, à 1'interface sédiments filons-couches, tels ceux à la base du filon-couche de Bourbeau dans le secteur de 1'apophyse de composition dunitique (minéralisation en pyrrhotine et argent). 
De plus, s`il y a création d’un aquifère et que celui-ci contient des fluides de haute température, ceux-ci tendront éventuellement à colmater les fractures avoisinantes par précipitation de silice lors de leur ascension (Facca et Tonani, 1967) et ainsi confiner cet aquifère. Une pression de fluide s’établissant dans ce milieu confiné, le système est placé sous contrainte, jusqu'au moment où la pression hydrostatique excède la résistance de la barrière, représentée par le filon-couche, à la rupture. La fracturation peut alors débuter. Les filons-couches du complexe de Cummings ont pu ainsi chenaliser des fluides, contenant potentiellement des minéralisations, le long de leurs bordures et fractures, comme le suggèrent Lewis (1955), Hodgson et Lydon (1977) et Campbel1 et al. (1981) jusqu’à des sites où la précipitation est possible. Cette précipitation étant causée par des variations de fo2, de fS2, de $\mathrm{pH}$, une rencontre avec un fluide météorique, etc.

En tenant compte de ces derniers points, la préexistence sur ces terrains de la majorité des minéralisations d’aspect stratiforme, prenant place dans la Formation de Blondeau et localisées, entre autres, à la base du filon-couche de Bourbeau ou moins couramment au sommet du filon-couche de Ventures, est 1'hypothèse de travail la plus probable. Ces points permettent aussi d'expliquer la présence locale de minéralisations filoniennes, en pyrrhotine et pyrite, de faible envergure et associées à de fortes silicification et carbonatisation qui aboutissent exceptionnellement à ces indices stratiformes. Par contre 
dans le cas des veines de pyrrhotine et de pyrite observées au sommet du filon-couche de Ventures et qui atteignent les sédiments susjacents de la Formation de Blondeau (?), sur le mont Bourbeau, la possibilité d'une contribution magmatique est en plus à considérer. La pyrrhotine, dans le membre gabbroìque silicifiée, y présente localement une texture en filet, quoique ce critère ne soit pas déterminant.

L'indice "Be1-3" représente un type de minéralisation occupant une zone grise entre un dépôt de type soit syn-volcanique (filonien et/ou magmatique ?) ou syn-tectonique. Les lentilles minéralisées contiennent de la chalcopyrite, de la pyrrhotine, de la pentlandite, de la magnétite, de la sphalérite, de la molybdénite et sont porteuses de valeurs ponctuelles intéressantes en argent et cobalt. Les roches encaissantes, de compositions pyroxénitiques et gabbroĩques, sont toutefois cisaillées et intensément carbonatisées.

Les diverses caractéristiques de ces lentilles minéralisées permettent d’envisager une origine magmatique. Les principaux points en faveur sont la présence de pentlandite (teneurs élevées en $\mathrm{Ni}$ et Co), les faibles altérations présentes (carbonatisation et chloritisation) à 1 'intérieur même de ces lentilles à 1'exception d’une mince zone de bordure de un à deux mètres dans les épontes et la disposition conforme des lentilles par rapport au litage magmatique observê à cet endroit dans le filon-couche de Ventures. Les points négatifs sont entre autres le caractère discontinu de ces minéralisations, leur position stratigraphique inhabituelle soit à $1^{\prime}$ intérieur d’un membre 
pyroxénitique, leur présence dans des cisaillements (les minéralisations sont-elles contemporaines du cisaillement qui les contient ou encore sont-elles encaissées dans une structure préférentielle réactivée par des mouvements tectoniques ultérieurs ?), la présence de sphalérite et de

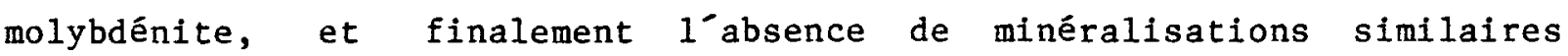
(éventuellement non encore reconnues?) dans le filon-couche de Ventures ailleurs dans le camp minier de Chibougamau.

Le caractère très déformé des minéralisations et leur fort degré de recristallisation soulignent par contre deux conditions importantes qui doivent être prises en considération dans l'élaboration d'un éventuel modèle: ces lentilles sont aussi vieilles que le cisaillement qui les contient, sinon davantage (planche XXVII (A) et (B)), et elles paraissent avoir subi les effets du métamorphisme régional (planche XXVII (C)). Ce dernier point, selon les travaux de Barrett et al. (1977) sur des dépôts archéens de $\mathrm{Cu}-\mathrm{Ni}$ de type volcanogène métamorphisé se retrouvant en Australie, rend très hasardeux toute hypothèse concernant leurs origines qui ne tiendrait pas compte de cette situation. $D^{-}$autre part, ces lentilles sont recoupées par plusieurs failles NNE du type "R", présentant des décrochements régulièrement senestres, et associées à la ceinture de cisaillement du lac Gwillim, d’âge tardi à post-tectonique (Dimroth et al., 1984a).

A quelques dizaines de mètres au sud des lentilles minéralisées de 1'indice "Bel-3" se trouve la trace d'une faille de chevauchement majeure, la faille du lac cummings. A la lumière des précédentes 
observations, deux hypothèses de travail peuvent néanmoins être tirées concernant la mise en place de ces lentilles: 1) ces minéralisations discontinues occupent en fait des fractures mineures sub-parallèles à la trace $d^{\prime}$ un chevauchement majeur, elles pourraient ainsi s’être mises en place tôt, au début de cet épisode tectonique, lors de I'amorce de la fracturation et avoir été déformées lors du mouvement culminant, soit le mouvement inverse lui-même. Ces minéralisations seraient ainsi au maximum d’âge syn-tectonique et contemporaines des épisodes de chevauchements régionaux; 2) ces lentilles sont d’origine magmatique, la coincidence voulant qư une faille de chevauchement prenne place à proximité est responsable de la création des cisaillements secondaires qui bordent ces minéralisations; la molybdénite et éventuellement la sphalérite provenant d'une contribution externe (le stock du lac line ?). Ces deux hypothèses permettent $d^{\prime}$ expliquer $d^{\prime}$ une manière plausible les figures d’interférences, le rubannement et les textures de recristallisation observées dans la partie massive des sulfures.

Cependant, Patterson et Watkinson (1984) ont documenté des caractéristiques similaires à 1'indice "Bel-3" dans le cas la mine Thierry, dans la ceinture volcanique archéenne $d^{\top}$ Uchi en Ontario. Ceux-ci en viennent à la conclusion de 1'existence de minéralisations magmatiques. A ce stade-ci de nos travaux, une telle hypothèse est également proposée pour expliquer 1'origine des lentilles de 1'indice "Be1-3".

$D^{\prime}$ autre part, il est bon de noter que sur nos 
terrains, les veines minéralisées ne se présentent jamais sur toute 1'épaisseur d’un filon-couche, quoique la fracturation puisse le faire localement. Ces veines se concentrent à la base ou au sommet des intrusifs, ce qui nous soumet certaines contraintes sur le mode de piègeage des minéralisations. Le fait aussi que les structures minéralisées se retrouvent dans des systèmes en tension, les évidences de cisaillement prononcé étant rares, est significatif du régime pré-tectonique de contraintes existant alors. Toutes ces minéralisations montrent d’ailleurs des textures de dêformation et de recristallisation poussées, associées vraisemblablement à 1'orogénèse Kénoréenne.

Du côté des minéralisations filoniennes syn-volcaniques porteuses $d^{\circ}$ or et d'argent, outre celles encaissées dans le filon-couche de Bourbeau, la plupart se retrouvent de façon préférentielle dans le membre inférieur du filon-couche de Roberge. Celui-ci étant une des premières roches-hôtes susceptibles de piéger d'éventuelles minéralisations en or et argent dans la partie supérieure du Groupe de Roy, ceci pourrait y expliquer leur présence. Les minéralisations en pyrite et pyrrhotine prennent place à des niveaux stratigraphiques qui apparaissent plus élevés, soit les filons-couches de Ventures et de Bourbeau. Les altérations entourant ces divers indices varient d'une silicification à une carbonatisation modérée à la base du filon-couche de Roberge. Vers le sommet du filon-couche de Ventures et à la base du filon-couche de Bourbeau, la carbonatisation et la silicification dominent plutôt sur la chloritisation. Toutes ces altérations demeurent néanmoins bien restreintes aux zones minéralisées. 
Finalement, les différents critères décrits précédemment nous permettent d`identifier certaines minéralisations stratiformes et filoniennes comme étant également d’origine volcanique. Il s’agit:

(1) de leurs orientations, généralement nord-sud à nord-nord-est et exceptionnellement nord-ouest,

(2) de leur composition, généralement riche en pyrite et pyrrhotine, ces sulfures étant stériles quand se retrouvant seuls ou encore présentant ponctuellement des valeurs en argent et localement en or quand associées soit à de la chalcopyrite et/ou de la sphalérite et/ou de la galène,

(3) de leurs extensions et dimensions restreintes dans le secteur du mont Bourbeau et de leurs positions dans la colonne stratigraphique dans ou aux bordures supérieures ou inférieures de corps intrusifs au comportement fragile que constituent les trois filons-couches du Complexe de Cummings, l'encaissant est souvent cisaillé,

(4) du type d'altération qui les entoure, soit une carbonatisation intense, dominant couramment sur la silicification et la chloritisation (la séricitisation étant genéralement faible) et

(5) du caractère déformé de ces minéralisations et des évidences de recristallisation intense des espèces sulfurées présentes.

En résumé, selon ces critères, les veines de sulfures massifs des indices des Dômes du Radar, 1'indice du lac Devilliers, 1'indice en pyrrhotine et argent en bordure nord de $1^{-}$apophyse du filon-couche de Bourbeau (mont Bourbeau), les veinules de pyrrhotine et pyrite au sommet du filon-couche de Ventures, le "Main Showing" et le prospect Taché seraient tous représentatifs de cette origine. A part le "Main Showing", de direction NNW, tous ces indices sont orientés NNE avec des pendages subverticaux à abrupts et de sens variables. Cette variété d'indices stratiformes, filoniens et magmatiques (?), tous comparables en âge, 
témoignent cependant de plusieurs événements distincts qui se sont succédés lors de cette époque.

6.3 Minéralisations filoniennes (tardi ou post-tectoniques) et leur mise en place

Ces minéralisations sont représentées de façon typique par $1^{\text {’indice }}$ du lac Ham, 1'indice d’argent et les différents indices minéralisés rencontrés dans le stock tonalitique du lac Line ou encore associés à celui-ci. Elles se distinguent des précédentes, syn-volcaniques, par le fait qu'elles occupent des fractures orientées régulièrement NW et rarement NNE-NE. Ces filonets se retrouvent à des niveaux stratigraphiques très divers, soit un filon-couche gabbröique de la Formation de Gilman, la base du filon-couche de Bourbeau et quelques sites dans la partie est du stock du lac Line.

L'indice du lac Ham montre des minéralisations se composant de magnétite, de pyrite, de chalcopyrite et de faibles valeurs en or et argent. Celles-ci occupent des fractures orientées NNW, montrant un minimum d'évidence de cisaillement. Ces fractures sont encaissées dans un filon-couche gabbroique de la Formation de Gilman. Ce filon-couche ne semble pas affecté par aucun décalage apparent de part et d'autre de ces cassures. L'altération dominante est une intense silicification à laquelle s'ajoute une chloritisation et une épidotisation d'intensités variables. Une faible carbonatisation se propage à 1'extérieur de cette zone minéralisée. La silicification est par contre étroitement restreinte à la zone minéralisée. Les assemblages de sulfures et d'oxydes présentent 
tous des évidences de recristallisation (ex: pyrite porphyroblastique) mais non de déformations marquées ou de rubannement.

L'indice d'argent, localisé à la base du filon-couche de Bourbeau, montre des cassures de direction $335^{\circ}$ à $350^{\circ}$, de pendages subverticaux à abrupts vers le SW. Celles-ci portent couramment des teneurs significatives en argent et plus rarement en or. Ces valeurs sont associées à des minéralisations en chalcopyrite et sphalérite. La silicification est encore ici étroitement restreinte aux zones minéralisées et la chloritisation dessine, en bordure immédiate des veinules, des figures de pseudo-bréchification. La séricitisation se remarque davantage à quelques distances des fractures, donnant à la roche une couleur blanc crème, la carbonatisation est de faible à nulle. Il $n^{-} y$ a pas de décalages apparents causés par ces fractures à la base du filon-couche de Bourbeau. Les minéralisations présentent des évidences de recristallisation (textures en points triples fréquentes).

Riverin et Hodgson (1980) et Knuckey et al. (1982) ont identifié, à

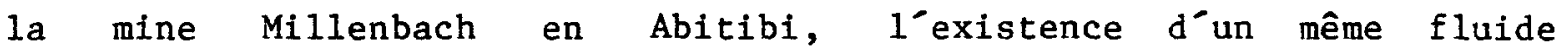
hydrothermal responsable successivement de chloritisations et de séricitisations intenses. Selon ces auteurs, la séricite apparait comme le premier minéral d’altération et sert de base à 1'élaboration du minéral d’altération subséquent, la chlorite, si les échanges entre ce fluide hydrothermal avec la roche-hôte et ce premier minéral d`altération ne sont pas interrompus pour une quelconque raison. 
Il y a donc simultanément deux fronts d’altérations distincts en progression qui se superpose sur une zone initialement silicifiée. Un premier front amène une séricitisation de plus ou moins grande étendue affectant de façon préférentielle les plagioclases, et un second, chloriteux, restreint d’abord à proximité du centre exhalatif ou aux fractures transportant les fluides hydrothermaux, se retrouve dans la matrice des volcaniclastites encaissantes et altère plus ou moins les reliques des plagioclases. Ce second front, tout comme le premier, peut

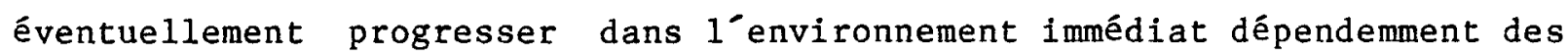
conditions suivantes: la quantité de fluide impliquée, la durée de cet événement hydrothermal et les conditions paléo-géographiques présentes

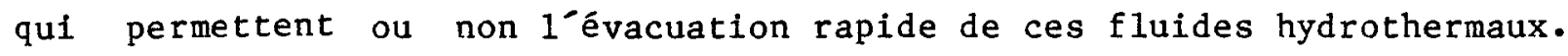
C'est 1'avance continue de ces deux fronts, la séricitisation étant de plus grande étendue que la chloritisation, qui dessine finalement sur I'affleurement cet aspect de pseudo-bréchification ( $\mathrm{planche} \mathrm{XIX).}$

Un cortège d’altérations grossièrement similaires à celles de 1 'indice d'argent se retrouvent apparemment dans le secteur du lac Devilliers. A cet endroit, certains lits grèseux présentent également des chloritisation et carbonatisation préférentielles, conférant à la roche un aspect pseudo-bréchiforme. Ces altérations, principalement une seconde phase de silicification et une forte chloritisation, se seraient alors superposées à une altération hydrothermale volcanogène précoce, une silicification et une carbonatisation accompagnées d'une chloritisation variable. Le potentiel de minéralisations filoniennes situées du côté ouest de ce secteur seraient ainsi fortement à examiner. $D^{-}$autre part, 
1 'aspect de cornéenne observé dans ces roches pourrait possiblement être dû au métamorphisme de contact du stock du lac Line, situé à moins de 150 mètres au sud-ouest.

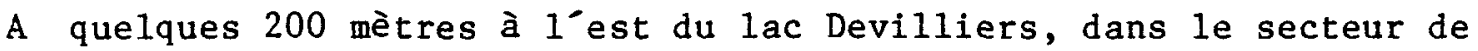
$1^{-}$apophyse dunitique du filon-couche de Bourbeau, des minéralisations en tous points similaires à celles de $1^{\text {'indice }} \mathrm{d}^{\prime}$ argent occupent ici une série de courtes fractures d'orientation NW et subverticales. Une fracture majeure, de direction NW, décale de façon dextre la base du filon-couche de Bourbeau. Les minéralisations se retrouvent par contre en bordure de cette faille, non directement dans celle-ci et régulièrement du côté des volcaniclastites de la Formation de Blondeau. Les minéralisations et la faille se poursuivent aussi du côté nord, dans le filon-couche de Bourbeau. Cette structure minéralisée NW est en tout point analogue à $1^{\prime}$ indice $d^{\circ}$ argent et $n^{\top}$ a jamais, selon nos travaux, été véritablement examinée par forage. A la lumière des résultats obtenus à 1'indice d'argent, son potentiel s'avère considèrable. Les textures de pseudo-bréchification, générées par les différents fronts de séricitisation et de chloritisation, sont encore ici très bien développées.

$\mathrm{Au}$ sud-est de cet indice, à moins de 40 mètres, un filonet orienté NNE, identifié plus tôt à un événement d’âge volcanique, porte une minéralisation constituée essentiellement de pyrite. La carbonatisation est intense dans et en bordure de la veine. La silicification et la chloritisation sont par contre de modérées à faibles. Les valeurs en 
cuivre, argent et or $y$ sont de très faibles à négligeables. Ceci, de même que les types d`altérations présentes contrastent fortement avec 1'indice adjacent, réputé d’âge tardi à post-tectonique, encaissé dans des fractures NW. La position stratigraphique de ces deux indices comportent toutefois plusieurs similitudes, ceux-ci se retrouvant soit à la base ou dans les bordures faillées d"un filon-couche.

Le stock tonalitique du lac Line présente divers indices dans sa partie est. Au sud de la Baie du Cran Penché, des veinules de quartz, minéralisées entre autres en chalcopyrite, sphalérite, pyrite et molybdénite, occupent des fractures d'orientation NE. La silicification est naturellement forte en bordure de ces veines. Des disséminations en pyrite et plus rarement en chalcopyrite se retrouvent même à une cinquantaine de mètres de ces veines dans de très minces fractures discontinues, encaissées dans la tonalite massive. Une faible séricitisation (forte valeur en $\mathrm{K} 20$ ) se présente conjointement à ces dernières.

Au nord-ouest du lac Ham, des filonets minéralisés en chalcopyrite, en pyrite et localement en magnétite ont donné des valeurs significatives en argent. Ces filonets, encaissés dans une tonalite, occupent de courtes fractures orientés NW, de pendages variables, inférieurs à une dizaine de mètres de longueur. La silicification et la chloritisation sont fortes en bordures des veines minéralisées. Leurs intensités décroissent radicalement à moins de deux ou trois mètres de celles-ci. 
Sur 1'indice des Dômes du Radar au mont Bourbeau, des dykes porphyriques en feldspath et quartz sont minéralisés en pyrite, en chalcopyrite et en molybdénite. Ces dykes sont orientés $E-W$ avec un pendage prononcé vers le nord. Une fracture, ayant sa trace selon une direction NNE, est également porteuse d’une minéralisation en molybdénite (planche XXI (C)). Celle-ci baigne dans un assemblage de quartz et de carbonates. L'altération en bordure de ces dykes et de cette fracture est minimum. Une association entre ces dykes et le stock tonalitique du lac Line ou encore un éventuel centre felsique volcanique demeure pour le moment dans le domaine des spéculations. Par contre, 1'orientation E-W de ces dykes est sub-parallèle à la trace de la faille du lac Cummings, qui se présente à moins de 75 mètres du côté nord, d’où 1'idée que cette structure en chevauchement aurait pu partiellement diriger la mise en place de ces dykes. Une origine syn à tardi-tectonique (suggérée de plus par 1'absence d'une schistosité pénétrative) semble donc à ce stade-ci des travaux 1'hypothèse la plus probable pour ces dykes. Ceci tendrait à signaler une association éventuelle avec le stock tonalitique du lac Line.

\footnotetext{
Ces minéralisations filoniennes tardi à post-tectoniques se distinguent ainsi des précédentes d’origine volcanique par les caractéristiques suivantes:
}

(1) 1'orientation régulière des fractures minéralisées, soit NW et plus rarement NNE à NE,

(2) les types d'altérations présents: une chloritisation et une séricitisation intenses associées à une silicification variable, souvent de grande étendue, et à une carbonatisation de modérée à faible, étroitement restreinte aux épontes 
des filons,

(3) 1'absence de cisaillement développé de façon systếmatique,

(4) les types de minéralisation: chalcopyrite, sphalérite, magnétite, et molybdénite associées à des valeurs significatives en argent et localement en or,

(5) le type et la position stratigraphique des roches hôtes: soit de préférence dans ou aux bordures d'une roche au comportement ductile (par exemple une masse ignée telle un filon-couche ou une tonalite) et

(6) les évidences de recristallisation couramment rencontrées dans les assemblages sulfures et oxydes, sans signe de déformations marquées.

Ces derniers points traduisent vraisemblablement $1^{\text {'existence }} \mathrm{d}^{\text {'un }}$ événement thermique postérieur à 1'orogénèse Kénoréenne et sont en accord avec les résultats des travaux préliminaires de Thorpe et al. (1981 et 1984) et de Guha (1984). Le stock tonalitique du lac Line, d’âge tardi-tectonique, s'avère dans cette région comme la manifestation possible d’un tel événement.

D'autre part sur le mont Bourbeau, Thorpe et al. (1984) ont également signalé une veine minéralisée en galène s'identifiant, d’après sa composition isotopique en plomb, soit à un événement minéralisateur distinct protérozoľque ou soit encore à une remobilisation d’âge protérozoĩque d’une minéralisation préexistante. Ceci souligne néanmoins la présence dans cette région et à cette époque de sources de minéralisations (d'une nature non définie pour le moment), de mécanismes de transport et de contextes aptes à piéger ces nouvelles minéralisations ou à diffuser celles qui étaient préexistantes. 
6.4 Considérations sur les modèles précédents et implications pour 1 'exploration minière.

Finalement, à partir des différents indices minéralisés décrits précédemment, il semble se dégager deux périodes distinctes pour la mise en place de ces dépôts. Une première période serait globalement contemporaine à 1'activité volcanique. Le dépôt du lac Berrigan (zinc-plomb-argent-or), daté autour de 2,72 G.A. (Thorpe et al., 1981 et 1984 ) et les différents indices filoniens rencontrés dans le Complexe de Cummings (tableau 4) en seraient de très bons exemples. Une seconde période, possiblement tardi ou post-tectonique (Kénoréen) comprendrait pour sa part un dépôt du type de $I^{\prime}$ indice d’argent, situé à la base du filon-couche de Bourbeau, ou encore du type du lac Ham.

Du côté des minéralisations volcaniques, les meilleurs indices se retrouvent à la base du filon-couche de Roberge; il s’agit du "Main Showing" et du prospect Taché. Des valeurs significatives en or et argent y sont rapportées. Les fractures minéralisées semblent par contre de courtes extensions et discontinues en surface. Elles sont affectées à divers degrés et tronquées par des événements tectoniques ultérieurs, ce qui a compliqué leur configuration et la recherche de leur extension potentielle.

I1 y aurait ainsi tout intêrêt à considérer le filon-couche de Roberge comme représentant le premier piège structural potentiel à la base du Complexe de Cummings et permettant la fixation des diverses minéralisations volcaniques pré-plissement. Des guides à 1'exploration pourraient être la recherche de larges zones altérées dans ce 
filon-couche, amenuisant ou rendant discontinue sa réponse magnétique sur les différents levés géophysiques compatibles, entre autres aéromagnétiques; la proximité de structures syn-volcaniques reconnues, par exemple le bassin d'effondrement en bordure est du prospect Taché (Pilote, 1986) ou encore la présence de faciès volcaniclastiques de types proximaux dans les Formations encaissantes de Blondeau et de Gilman.

L’indice "Be1-3", encaissé dans le filon-couche de Ventures, souligne pour sa part l'intérêt qui existe de retrouver des minéralisations similaires, d’origine magmatique (?), ailleurs dans le camp minier. Sur cet indice, les lentilles minéralisées se situent du côté nord de ce chevauchement, dans des cisaillements qui luí sont sub-parallèles. Un point crucial à déterminer reste à savoir si ces cisaillements sont contemporains et associés d'une manière quelconque à ces minéralisations ou encore slils leur sont plus tardifs. Des minêralisations tardives par rapport à ces structures pourraient même les avoir éventuellement empruntées ou encore y avoir été piégées.

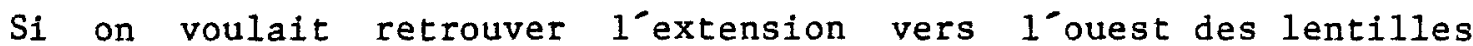
minéralisées des indices "Bel-3" et du lac Devilliers, au delà du stock tonalitique du lac Line, il faudrait chercher dans le segment se situant au nord da la faille du lac Cummings. Ce segment et la faille inverse elle-même $n^{\prime}$ affleurent pratiquement pas. Ils devraient selon notre interptétation se retrouver exactement sous les eaux du lac Larone. Ce secteur, comprenant la Formation de Blondeau et le Complexe de Cummings, est donc particulièrement à privilêgier dans la recherche de 
minéralisations, volcaniques et de tardi à post-tectoniques, similaires à celles rencontrées du côté est du lac Line.

Le filon-couche de Bourbeau s’avère un site préférentiel pour le piégeage de minéralisations de tous âges. Par contre, les minéralisatons du type tardi à post-tectoniques semblent être les plus intéressantes, à cause des teneurs en argent et localement en or qu'elles contiennent. La présence du stock tonalitique du lac Line, situé à proximité, est à considérer comme source possible des minéralisations et des altérations associées, soit de fortes silicification, séricitisation et chloritisation ponctuelles. Ainsi le filon-couche de Bourbeau ne constituerait pas en lui-même une source de minéralisation majeure mais plutôt un réceptacle idéal, dépendant des conditions géologiques locales et régionales, pour le piégeage d’éventuelles minéralisations relachées par divers systèmes extérieurs, quels que soient leurs âges.

Du côté stratigraphique, la plus grande variété d’indices minéralisés de tous âges se retrouve sur le mont Bourbeau et particulièrement dans ou à proximité des différents filons-couches du Complexe de Cummings. Dans ce secteur, les signes d'un centre volcanique felsique sont observés et son extension est interprétée se poursuivre soit vers $1^{\text {e }}$ est ou vers 1 e nord. Ce centre pourrait représenter $1^{\circ}$ apex de l'édifice volcanique mafique proposé dans les environs de la plaine où se situe la ville de Chibougamau (Allard, 1984).

Un tel centre expliquerait de façon simple I'abondance de 
minéralisations volcaniques dans ce secteur. $D^{-}$autre part, la proximité du stock du lac Line pourrait y expliquer la présence de minéralisations tardives. Ainsi la superposition de ces deux événements d’âge dictinct dans un même secteur, à savoir un centre volcanique et un intrusif tardif porteur de minéralisations, aurait amené dans la région du mont Bourbeau une densité élevée de différents indices minéralisés.

Toutes ces caractéristiques nous portent à croire que des minéralisations tardi à post-tectoniques, non encore reconnues, devraient également se retrouver dans le secteur du lac Larone dans des structures préférentiellement d'orientation NW, au sud et principalement au nord de la trace de la faille du lac Cummings.

Des minéralisations d’âge post-tectonique se retrouvent aussi dans la Formation de Gilman, soit 1'indice du lac Ham. Dans la recherche d'éventuelles minéralisations de ce type, il y a donc lieu de considérer également les massifs au comportement fragile, comme ceux représentés par des filons-couches et possiblement par certaines coulées volcaniques massives de la Formation de Gilman.

Le stock tonalitique du lac Line recoupe la faille du lac Cummings et plusieurs failles de direction NE, de type "R". Par contre, la faille

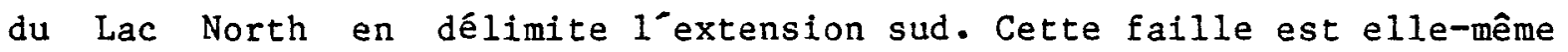
recoupée par quelques grandes cassures d'orientation NE de décrochements dextres et NW de dêcrochements variables, ces dernières affectant elles-aussi ce pluton. Ceci tendrait à lui indiquer une mise en place 
tardi à post-kénoréenne mais antérieure au protérozoĩque. Ces observations nous signalent ainsi des relations d’âge à considérer sur le moment où ont pu prendre place les minéralisations en molybdénite, chalcopyrite et argent qui lui sont associées, encaissées dans des veines de quartz. Finalement, le rôle qu’a pu jouer ce pluton, face aux déformations régionales et dans I'instauration, la propagation ou la réactivation de certains réseaux de failles demeure non quantifiable pour le moment.

Cet intrusif tonalitique est spécialement intéressant sur trois points: premièrement, la présence d'une phase locale de syénite

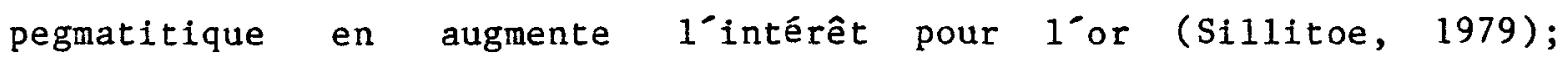
deuxièmement, des teneurs localement significatives en argent y sont rencontrées, au nord-ouest du lac Ham (tableau 3); et troisièmement, il représenterait la source de chaleur nécessaire pour la composante tardive des minéralisations rencontrées sur ce terrain.

Ce stock, tardif du point de vue tectonique, pourrait également s’avérer la source d’une certaine partie des différentes minéralisations en argent et localement en or, rencontrées dans le secteur du mont Bourbeau à l'intérieur de plusieurs cassures orientées NNW. Il est de plus intéressant de noter que ce sont les fractures globalement de direction NW qui affectent de la façon la plus intense cet intrusif. Ces réseaux de cassures préférentiels auraient chenalisé les fluides minéralisateurs, d’origine magmatique (?) ou autre, le long de diverses lithologies. Encore une fois, les différents filons-couches du Complexe 
de Cummings auraient agi comme piège structural préférentiel pour ces fluides, comme ils $1^{\prime}$ avaient fait auparavant pour les fluides hydrothermaux d'origine volcanique.

En résumé, 1'aspect des cassures minéralisées, leur orientation préférentielle et la composition des différentes minéralisations présentes et des cortèges d'altérations associés nous ont servi à établir ce modèle de travail préliminaire. Des travaux ultérieurs, notamment de nature isotopique, ou encore sur 1'environnement représenté par le stock du lac Line, pourront sans doute aider à résoudre plusieurs des problèmes soulevés ici. On doit cependant bien garder à 1'esprit que les conclusions de ces travaux ne laissent aucunement sous-entendre qu' un type de fracturation, d'orientation NW par exemple, est unique à une époque donnée. Il peut tout aussi bien se présenter avant qứaprès 1'orogénèse Kénoréenne. Par contre, 1'ampleur des structures filoniennes minéralisées, leur fréquence et la présence ou non de certaines minéralisations et altérations sont des caractères typiques qui permettent de discriminer 1'ampleur et la spécificité de chaque système de cassure, dans une région également spécifique . 
CHAPITRE 7

CONCLUSIONS

Les minéralisations présentes sur ce terrain se subdivisent en deux catégories:

(1) Volcaniques: -stratiformes pré ou

-filoniennes syn-Kénorêennes

(2) Filoniennes (tardi ou post-Kénoréennes).

Des contrôles structuraux et stratigraphiques ont fortement contrôlé la mise en place des différents dépôts minéralisés.

Ainsi, la majeure partie des indices prend place dans des structures favorables, soit les cassures à $1^{\prime}$ intérieur des différents filons-couches du Complexe de Cummings ou soit celles tout près de leur base (Bourbeau et Roberge) ou de leur sommet (Ventures).

Du côté stratigraphique, la plus grande variété d'indices minéralisés se retrouve sur le mont Bourbeau, où la proximité d'un centre volcanique felsique est fortement soupçonnée, celui-ci se situant soit vers $1^{\text {'est }}$ ou vers le nord. Le stock tonalitique du lac Line, lui-même porteur de diverses minéralisations, a pu contribuer pour sa part à certaines des minéralisations post-tectoniques rencontrées dans ce secteur.

Les minéralisations présentes dans le Complexe de Cummings montrent 
que celui-ci a agi comme piège structural et stratigraphique aussi bien pour les fluides hydrothermaux "volcanogènes" (pré et syn-Kénoréens) que pour les fluides tardifs (tardi à post-Kénoréens). Cependant la nature de ces fluides est encore incertaine. Ceci fait de ce complexe la meilleure cible à prospecter dans la région étudiée. Dépendamment du contexte géologique local et régional, et particulièrement s'il y a présence de zones d’altérations majeures ou de cassures favorables, son potentiel devrait être fortement à considérer ailleurs dans le district.

Des failles, d'orientations caractéristiques pour chaque type de minéralisation, ont largement contribué au piégeage des dépôts minéralisés. Les indices de nature filonienne s'étant révélés les plus intéressants du point de vue économique, les successions et rôles que ces cassures ont pu avoir dans la région deviennent particulièrement importants à déterminer pour 1'exploration minérale. Le tableau (2) suggère un modèle présentant 1'ordre d'apparition des divers réseaux de failles observées.

Le stock tonalitique du lac Line, d’âge post-tectonique, en plus d’être porteur lui-même de minéralisation, a pu avoir un rôle important à jouer, dans les patrons de fracturation et de déformation locaux. De plus, son rôle comme source de chaleur pour une certaine partie des fluides minéralisateurs tardifs, est fortement à envisager, quoique non quantifiable pour le moment. 


\section{REFERENCES}

Alexander, R.L., 1969 - Brosnan Canadian Mine Ltd., geological report on property, McKenzie township, Chibougamau area. Ministère Rich. Nat., Qué.; GM-24458, 12 p.

Allard, G.0., 1956 - The geology of a portion of McKenzie township, Chibougamau district, Quebec. Thèse de Ph.D. non-publiée, The Johns Hopkins Univ., Baltimore, Md., $168 \mathrm{p}$.

Allard, G.0., 1976 - Doré Lake Complex and its importance to Chibougamau geology and metallogeny. Ministère Rich. Nat., Qué.; DP-368, 446p.

Allard, G.0., 1978 - Pétrologie et potentiel économique du prolongement du silion de roches vertes de Chibougamau dans la Province de Grenville. Ministère Rich. Nat., Qué.; DPV $-604,44$ p.

Allard, G.0., 1979 - Prolongement du Complexe du lac Doré dans

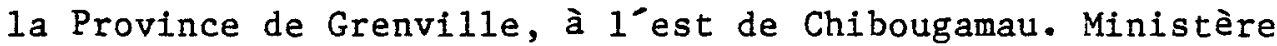
Rich. Nat., Qué.; DPV-685, 18 p.

Allard, G.0., 1981 - Quart sud-ouest du canton de Rinfret et partie du quart sud-est du canton de Lemoine - Relation avec le Front de Grenville. Ministère Energie Ressources, Qué.; DPV-759, 95 p.

Allard, G.0., 1982 - Géologie du quart nord-ouest du canton de Haüy. Ministère Energie Ressources, Qué.; Dpv-928, 57 p.

Allard, G.0., 1984 - General geology of the Chibougamau region. Dans J. Guha et E.H. Chown (eds), Chibougamau - Stratigraphy and Mineralization, C.I.M. Special Volume 34, pp. 5-19.

Allard, G.0. - Caty,.J.-L. - Chown, E.H. - Cimon, J. - Gobeil, A. - Baker, D., 1979 - Stratigraphie et métallogénie de la région de Chibougamau. Assoc. Géol. Can. et Ass. Minéral. Can., livret-guide de 1'excursion B-1, 95 p.

Archambault, G. - Guha, J. - Tremblay, A. - Kanwar, R., 1984 Implications of the geomechanical interpretation of the Copper Rand Deposit on the Doré Lake shear belt. Dans J. Guha et E.H. Chown (eds), Chibougamau - Stratigraphy and Mineralization, C.I.M. Special Volume 34, pp. 300-318. 
Archambault, G. - Poitras, A. - Guha, J. et autres, 1986 - A geomechanical analysis of the Dore Lake and McKenzie Narrow shear belts, Chibougamau area, Quebec. En préparation.

Archer, P., 1983 - Interprétation de 1'environnement volcanosédimentaire de la Formation de Blondeau dans la section stratigraphique du lac Barlow, Chibougamau. Mémoire de M. Sc. A. non publ., Université du Québec à Chicoutimi, 160 p.

Archer, P., 1984 - Interpretation of the volcano-sedimentary environment of the Archean Blondeau Formation, Barlow Lake section Chibougamau. Dans J. Guha et E.H. Chown (eds), Chibougamau - Stratigraphy and Mineralization, C.I.M. Special Volume 34, pp. 92-106.

Avramtchev, L. - Lebe1-Drolet, S., 1981 - Cartes des gites minéraux du Québec, Région de 1'Abitibi. Ministère Energie Ressources, Québec; DPU-744.

Barlow, A.-E. - Faribault, F.-R. - Gwillim, J.-C., 1911 Rapport sur la géologie et les ressources minérales de la région de Chibougamau (Québec). Ministère de la colonisation, des mines et des pêcheries; rapport de la Commission Minière de Chibougamau, $243 \mathrm{p}$.

Barrett, F.M. - Binns, R.A. - Groves, D.I. - Marston, R.J. McQueen, K.G., 1977 - Structural history and metamorphic modification of Archean volcanic-type nickel deposits, Yilgarn block, Western Australia. Econ. Geol. v. 72, pp. 1195-1223.

Bélanger, J. - Guha, J. - Coulombe, A. - Carignan, J., 1984 - The "8-5" zone, Cooke Mine, Chapais: a volcanogenic massive sulfide deposit in the Blondeau Formation. Dans J. Guha et E.H. Chown (eds), Chibougamau - Stratigraphy and Mineralization, C.I.M. Special Volume 34, pp. 271-287.

Bouchard, G. - Guha, J. - Zuckerkandel, W. - Carignan, J. Archambault, G., 1984 - The Gwillim Gold Mine and its geological setting. Dans J. Guha et E. Chown (eds), Chibougamau - Stratigraphy and Mineralization, C.I.M Special Volume 34 , pp. 370-377.

Bouchard, G., 1986 - Minéralisations aurifères à la mine Gwillim et les environs. Mémoire de M. Sc. A. (en préparation), Université du Québec à Chicoutimi.

Bradley, J., 1965 - Intrusions of major dolerite sills. Trans. Roy. Soc. New Zealand, v. 3, pp. 25-35.

Campbe11, I.H. - Franklin, J.M. - Gorton, M.P. - Hart, T.R. Scott, S.D., 1981 - The role of subvolcanic sills in the generation of massive sulfide deposits. Econ. Geol., v. 76 , pp. $2248-2253$. 
Carignan, J. - Dembele, Y. - Guha, J. - Gobert, A. - Fowler, A., 1984 - Basic geochemical profile of the Blondeau Formation in the Barlow Lake stratigraphic section. Dans J. Guha et E.H. Chown (eds), Chibougamau - Stratigraphy , and Mineralization, C.I.M. Special Volume 34, pp. 107-119.

Caty, J.-L., 1977 - Demie est du canton de Richardson. Ministère Rich. Nat., Qué.; DPV-447, 11 p.

Caty, J.-L., 1979 - Demie ouest du canton de Bignel1. Ministère Rich. Nat., Qué.; DPV-678, 22 p.

Chown, E.H., 1984 - Mineralization controls in the Aphebian Formation; Chibougamau, Mistassini and Otish areas. Dans J. Guha et E.H. Chown (eds), Chibougamau-Stratigraphy and Mineralization, C.I.M. Special Volume 34, pp. 229-243.

Cimon, J., 1976 - Géologie du canton de Queylus (NE), comté d'Abitibi-est. Ministère Rich. Nat., Qué.; DPV-439, 34 p.

Cimon, J., 1977 - Quart sud-est du canton de Queylus. Ministère Rich. Nat., Qué.; DPV-448, 33 p.

Cimon, J. - Gobeil, A., 1976 - The Stella Formation: its implication for the genesis and the relative age of mineralization in the Chibougamau mining camp. C.I.M. Bu1l., Réunion Annue1le, v.69, no. 767, p.96 (résumé).

Cronsilver, S., 1957 - Taché Lake Mines Ltd., diamond drill hole logs and assay results, McKenzie township, Quebec. Ministère des Mines, Qué.; GM-4777-B, 45 p.

Daigneault, R. - Allard, G.0., 1984 - Evolution tectonique $d^{\prime}$ une portion du sillon de roches vertes de Chibougamau. Dans J. Guha et E.H. Chown (eds), Chibougamau - Stratigraphy and Mineralization, C.I.M. Special Volume 34, pp. 212-228.

Dallmeyer, R.D. - Maybin, A.H. - Durocher, M.E., 1975 Timing of kenorean metamorphism in the eastern Abitibi greenstone belt, Quebec: Evidence from 40Ar/39Ar ages of hornblende and biotite from post-kinematic plutons. Can. J. Earth Sci., v. 12, pp. 1864-1873.

Dimroth, E. - Imreh, L. - Rocheleau, M. - Goulet, N., 1982 Evolution of the south-central part of the archean Abitibi-belt, Quebec. Part 1: stratigraphy and paleogeographic model. Can. J. Earth Sci., v. 19, pp. 1729-1758.

Dimroth, E. -Mueller, W. - Rocheleau, M. - Archer, P. Jutras, M.- Piché, M. - Simoneau, P. - Carignan, J. Chown, E.H. - Guha, J. - Goulet, N. - Allard, G.0. - 
Franconi, A. - Gobeil, A., 1983 - Stratigraphie et évolution du bassin de transition entre les Groupes de Roy et d'Opémisca, région de Chibougamau-Chapais. Ministère Energie Ressources, Qué.; DV 83-11, pp.21-34.

Dimroth, E. - Archambault, G. - Goulet, N. - Guha, J. Mueller W., 1984a - A mechanical analysis of the late Archean Gwillim Lake shear belt, Chibougamau area, Quebec. Can. J. Earth Sci., v. 21, pp 963-968.

Dimroth, E. - Rocheleau, M. - Mueller, W., 1984b - Paleography, isostasy and crustal evolution of the Archean Abitibi Belt: a comparaison between the Rouyn-Noranda and ChibougamauChapais areas. Dans J. Guha et E.H. Chown (eds), Chibougamau - Stratigraphy and Mineralization, C.I.M. Special Volume 34, pp. 73-91.

Dimroth, E. - Rocheleau, M.- Mueller, W. - Archer, P. Brisson, H. - Fortin, G. - Jutras, M. - Lefebvre, C. Pilote, P.: - Simoneau, P.,1985a - Paleogeographic and paleotectonic response to magmatic processes: a case history from the Archean sequence in the Chibougamau area, Quebec. Geologische Rundschau, v. 75, pp.11-32.

Dimroth, E. - Mueller, W. - Daigneault, R. - Brisson, H. Poitras, A. - Rocheleau, M., 1985b - Diapirism during regional compression: The structural pattern in the Chibougamau region of the Archean Abitibi Belt, Quebec. Geologische Rundschau, Sonderband fuer H. Cloos (sous presse).

Dubé, C.-Y., 1983 - Géologie des cantons de Lamarck et de Guettard. Ministère Energie Ressources, Qué.; dans Rapports d'étape des travaux en cours à la division du précambrien, ET 82-01, pp.133-161.

Duquette, G., 1970 - Stratigraphie de 1'Archéen et relations métallogéniques dans la région de Chibougamau. Ministère Rich. Nat., Qué.; Etude spéciale 8, 16 p.

Duquette, G., 1972 - tiré de: Allard, G.0., 1972 - Geology of the northeast quarter of Scott township. Ministère Rich. Nat., Qué.; DP-118, 18 p.

Duquette, G., 1976 - North half of McKenzie and Roy townships and northwest quarter of McCorkill township. Ministère Rich. Nat., Qué.; DP-357, 126p.

Duquette, G., 1982 - Demie nord des cantons de McKenzie et de Roy et quart nord-ouest du canton de McCorkil1. Ministère de 1'Energie et des Ressources, Québec; DP-837, $72 \mathrm{p}$. 
Einsele, G. - Gieskes, J.M. - Curray, J. - Moore, D.M. Aguayo, E. - Aubry, M.-P. - Fornari, D. - Guerrero, J. Kastner, M. - Kelts, K. - Lyle, M. - Matoba, Y. Molina-Cruz, A. - Niemitz, J. - Rueda, J. - Saunders, A. Schrader, H. - Simoneit, R. - Vacquier, V., 1980 - Intrusion of basaltic sills into highly porous sediments, and resulting hydrothermal activity. Nature, v. 238, pp.441-445.

Facca, G. - Tonani, F., 1967 - The self-sealing geothermal field. Bu11. Volcanol., v. 30, pp.271-273.

Fahrig, W.H. - Gaucher, E.H. - Larochelle, A., 1965 Paleomagnetism of diabase dykes of the Canadian Shield. Can. J. Earth Sci., v. 2, pp. 278-298.

Francis, E.H., 1982 - Magma and sediment - I, emplacement mechanism of late Carboniferous dolerite sills in Northern Britain. J. Geol. Soc. London, v. 139, pp. 1-20.

Gibson, H.L. - Watkinson, D.H., 1979 - Silicification in the Amulet "rhyolites" Formation, Turcotte Lake section, Noranda area, Quebec. Dans Current Research, part B, Geol. Surv. Can., Paper 79-1B, pp. 111-120.

Gobei1, A. - Racicot, D., 1983 - Carte lithostratigraphique de la région de Chibougamau. Ministère Energie Ressources, Qué.; Mémoire MM 83-2.

Goodwin, A.M. - Ridler, R.H., 1970 - The Abitibi orogenic belt. Dans A.J. Baer (ed), Symposium on basins and geosynclines of the Canadian Shield. Geol. Surv. Can.; Special Paper $70-40$, pp. 1-30.

Graham, R.B., 1967 - Report on geology of Amalgamated BeauBelle Mines Ltd., Belle group, McKenzie township, Quebec. Ministère Rich. Nat. Qué.; GM-19028, 17 p.

Guha, J. - Archambault, G. - Leroy, J., 1983 - A correlation between the evolution of mineralizing fluids and the geomechanical development of a shear zone as illutrated by the Henderson 2 Mine, Quebec. Econ. Geol., v. 78, pp. 1605-1618.

Guha, J., 1984 - Hydrothermal systems and correlations of mineral deposits in the Chibougamau mining district. Dans J. Guha and E.H. Chown (eds), Chibougamau - Stratigraphy and Mineralization, C.I.M. Special Volume 34, pp. 517-534.

Henry, R.L. - Allard, G.0., 1979 - Formation ferrifère du lac Sauvage, cantons de McKenzie et de Roy, région de Chibougamau. Ministère Rich. Nat., Qué.; DPV-593, 90 p. 
Hodgson, C.J. - Lydon, J.W., 1977 - Geological setting of volcanogenic massive sulfide deposits and active geothermal systems: some implications for explorations. C.I.M. Bul1., v.70, No. 10, pp. 95-106.

Knuckey, M.J. - Comba, C.D.A. - Riverin, G., 1982 - Structure, metal zoning and alteration at the Millenbach deposit, Noranda, Quebec. In Precambrian sulfide deposits, Geol. Ass. Can. Spec. Paper 25, pp. 255-296.

Landry, J., 1984 - Etude de la minéralisation dans le secteur du lac Berrigan, canton de McKenzie. Projet de fin d'études non publié, Université du Québec à Chicoutimi, 56 p.

Large, J., 1977 - Chemical evolution and zonation of massive sulfide deposits in volcanic terrains. Econ. Geol., v.72, pp. 549-572.

Lefebvre, C., 1986 - Volcanologie et sédimentologie de la Formation de Blondeau, Chibougamau. M. Sc. A. (en préparation), Université du Québec à Chicoutimi.

Lewis, D.V., 1955, - Relationships of ore bodies to dikes and sills. Econ. Geol., v. 50, pp. 495-516.

Long, D.G.L., 1974 - Glacial and paraglacial genesis of conglomeratic rocks of the Chibougamau Formation (Aphebian), Chibougamau, Quebec. Can. J. Earth Sci., v.11, pp. 1236-1252.

McMillan, R.H., 1972 - Petrology, geochemistry and wallrock alteration at Opemisca: a vein copper deposit cross cutting a layered Archean ultramafic-mafic sill. Unpubl. Ph.D thesis, University of Western Ontario, London, Ont., 168 p.

Mawdsley, J.B. - Norman, G.W.H., 1935 - Chibougamau Lake maparea, Quebec. Geol. Surv. Can.; Memoir 185, 95 p.

MER, 1979 - Cartes de compilation géoscientifique de la région de Chibougamau - 32 G/16. Ministère Rich. Nat., Qué.; DPV-701.

MRN, 1978 - Résultats d'un levé INPUT dans la région d'Opémisca. Ministère Rich. Nat., Qué.; DP-587.

Mueller, W. - Dimroth, E., 1984 - Sedimentology and depositional history of the Blondeau and Chebistuan Formations in the Waconichi syncline, Chibougamau, Quebec: Dans J. Guha et E. Chown (eds), Chibougamau Stratigraphy and Mineralization, C.I.M. Special Volume 34 , pp. 137-152. 
Patterson, G.C. - Watkinson, D.H., 1984 - The geology of the Thierry Cit-Ni Mine, Northwestern Ontario. Can. Mineral. v. 22, pp. 3-11.

Piché, M., 1984 - The Haüy Formation: subaerial volcanism in a braided stream environment. Dans J. Guha et E.H. Chown (eds), Chibougamau - Stratigraphy and Mineralization, C.I.M. Special Volume 34, pp. 153-162.

Pilote, P., 1983 - Etude gîtologique et métallogénique de la région du Mont Bourbeau, Chibougamau. Ministère de 1'Energie et des Ressources, Québec; rapport préliminaire, DP 83-9.

Pilote, P., 1984 - Gîtologie et stratigraphie de la région du lac Berrigan, Chibougamau. Ministère de I'Energie et des Ressources, Québec; rapport préliminaire, DP 84-17.

Pilote, P., 1986 - Stratigraphie, structure et gitologie de la région du lac Berrigan. Ministère de 1-Energie et des Ressources, Québec; rapport final (sous presse).

Pilote, P. - Guha, J. - Dimroth, E. - Gobeil, A., 1984 a Stratigraphic positions of mineralized occurences in the Mount Bourbeau and Lac Berrigan regions and their significance. Dans J. Guha et E.H. Chown (eds), Chibougamau Stratigraphy and Mineralization, C.I.M. Special Volume 34, pp. 288-299.

Pilote, P. - Tremblay, A. - Guha, J., 1984b - Stratigraphy and mineralization in the Roy Group and the Cummings Complex, Chibougamau Syncline, Mount Bourbeau area. Dans Chibougamau - Stratigraphy and Mineralization, livret-guide d'excursions, la Division de la Géologie de I'ICM, pp. 159-189.

Plimer, I.R., 1978 - Proximal and distal stratabound ore deposit. Mineral. Deposita, v. 14, pp. 345-353.

Poitras, A., 1984 - Caractérisation géochimique du Complexe de Cummings, région de Chibougamau-Chapais, Québec. Mémoire de maîtrise non publié, Université du Québec à Montréal, 152 p.

Racicot, D., 1980 - Géochimie et métallogénie de la partie orientale du Pluton de Chibougamau. Ministère Energie Ressources, Québec; DPV-758, 20 p.

Racicot, D., 1981 - Géochimie et métallogénie des parties occidentales et centrales du pluton de Chibougamau. Ministère Energie Ressources, Québec; DPV-834, 26 p. 
Racicot, D. - Chown, E.H. - Hanel, T., 1984 - Plutons of the Chibougamau-Desmaraisville belt; a preliminary survey. Dans J. Guha et E.H. Chown (eds), Chibougamau - Stratigraphy and Mineralization, C.I.M. Special Volume 34, pp. 178-197.

Racine, M., 1984 - Rapport d'avancement des travaux, propriété Bel, canton de McKenzie, Chibougamau. Les Mines Camchib Inc.; rapport interne.

Retty, J.A., 1929 - Canton de McKenzie, région de Chibougamau, Québec; Service des Mines du Québec; rapport annuel, partie D, pp. 52-84.

Richardson, J., 1870 - Rapport des opérations. Comm. Géol. Can., 1870-71.

Riverin, G. - Hodgson, J., 1980 - Wall-rock alteration at the Millenbach Cu-Zn mine, Noranda, Quebec. Econ Geol. v. 75, pp. 424-444.

Sillitoe, R.H., 1979 - Some thoughts on gold-rich porphyry copper deposits. Mineral. Deposita (Berl.), v. 14, pp. 161-174.

Smith, J.R. - Allard, G.-0., 1960 - Moitié sud du canton de McKenzie. Ministère des Mines, Québec; RG-95, 71 p.

Tait, L. - Pilote, P. - Chown, E.H., 1985 - Géologie de la région du lac à 1 Eau Jaune. Ministère Energie Ressources, Qué.; rapport préliminaire (sous presse).

Tchalenko, J.S., 1968 - The evolution of kink bands and the development of compression textures in sheared clays. Tectonophysics, v.6, pp.159-174.

Thorpe, R.I. - Guha, J. - Cimon, J., 1981 - Evidence from lead isotopes regarding the genesis of ore deposits in the Chibougamau region, Quebec. Can. J. Earth Sci., v. 18 , pp. $708-723$.

Thorpe, R.I. - Guha, J. - Franklin, J.M. - Loveridge, W.D., 1984 - Use of the Superior Province lead isotope framework in interpreting mineralization stages in the Chibougamau district. Dans J. Guha et E.H. Chown (eds), Chibougamau - Stratigraphy and mineralization, C.I.M. Special Volume 34, pp. 496-516.

Trudeau, Y., 1981 - Pétrographie et géochimie des roches du secteur êrvironnant de la mine Bruno, Chibougamau, Québec. Mémoire de M. Sc. A. non-publié, Université du Québec à Chicoutimi, $110 \mathrm{p}$. 
Trudel, P. - Cloutier, M.A., 1984 - Lithogeochemistry of the Gilman and Blondeau Formations in the Chibougamau region. Dans J. Guha et E.H. Chown (eds), Chibougamau - Stratigraphy and Mineralization, C.I.M. Special Volume 34, pp.120-136.

Wolhuter, L.E., 1962 - Géologie du quart sud-est du canton de Daubrée, comté d'Abitibi-est. Ministère des Mines, Québec; $\mathrm{RP}-474,16 \mathrm{p}$. 
ANNEXES 
ANNEXE 1

PLANCHE I

(A) Alternance de lits grèseux et argileux contenant des horizons graphiteux, riches en sulfures disséminés (pyrite).

(B) Alternance de lits silteux et grèseux d'épaisseurs millimétriques à centimétriques. Au sud du lac Devilliers, mont Bourbeau. 


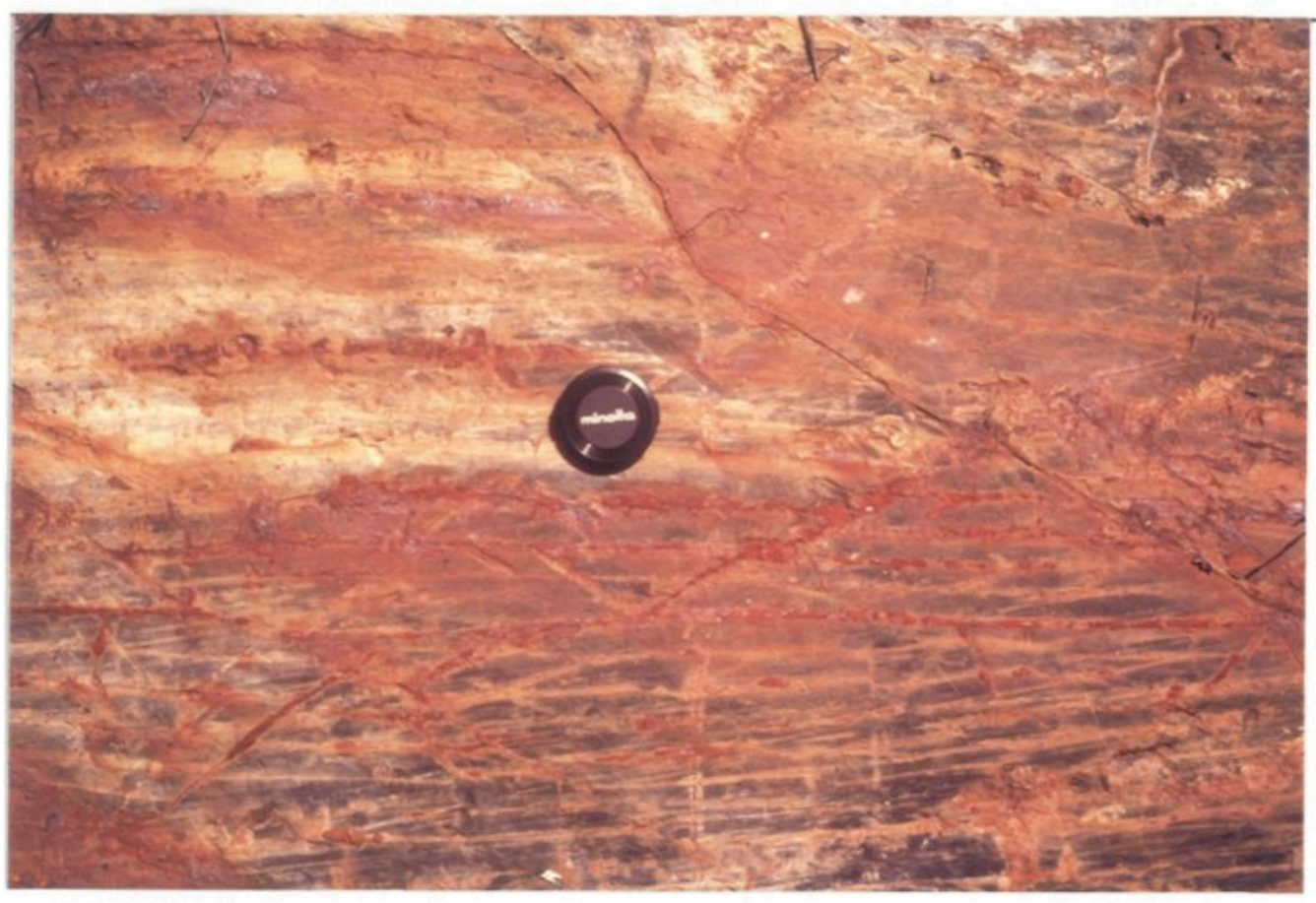

(A)

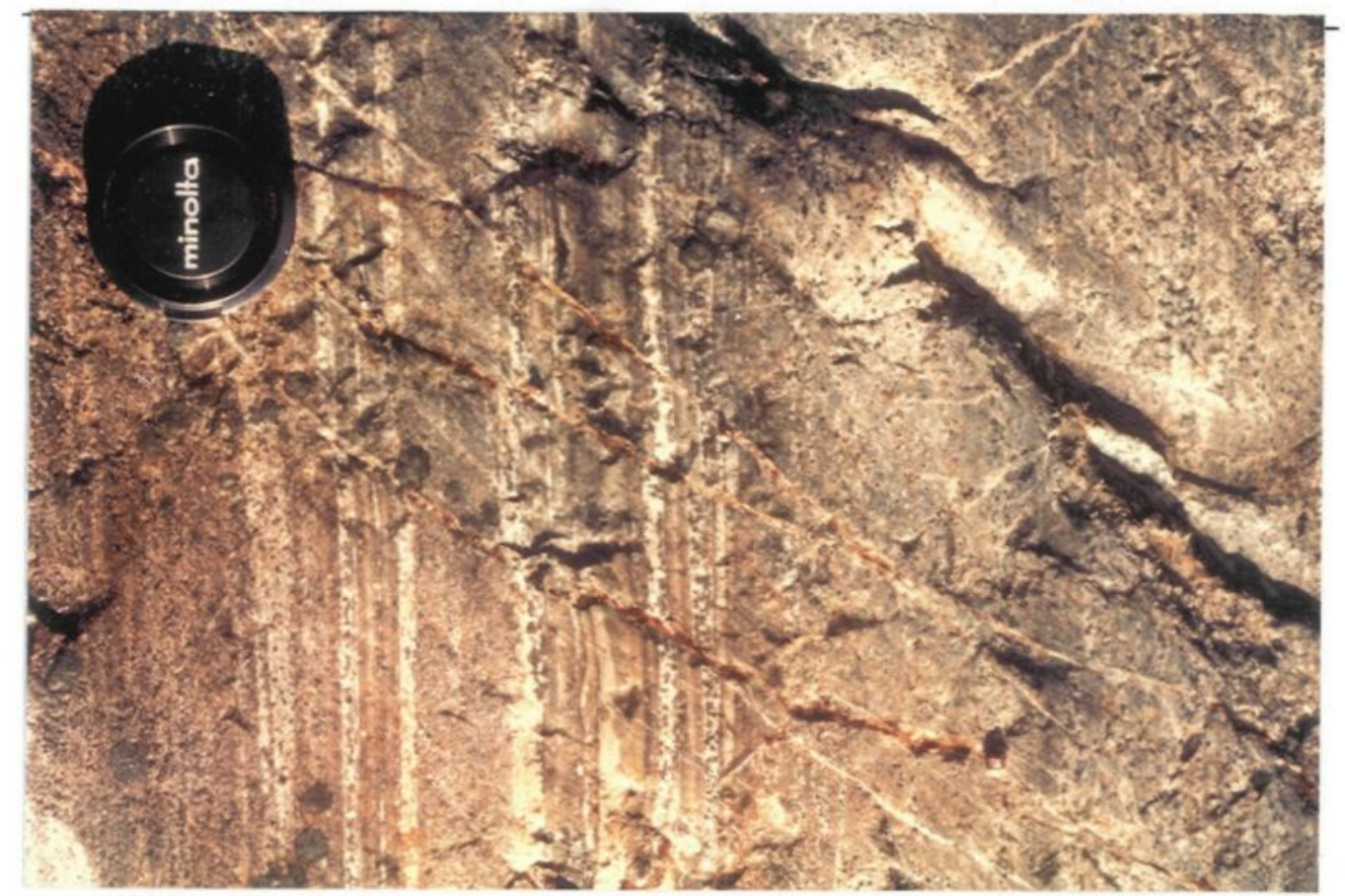


PLANCHE II

(A) Sédiment mixte contenant des fragments mafiques porphyriques en plagioclase incorporés à une matrice composée de cristaux libres de plagioclase et de quartz. A l'est du lac Devilliers, Formation de Blondeau. Lumière naturelle.

(B) Coulée pyroclastique oligolithilogique composée de cristaux libres de plagioclase séricitisés, de quartz résorbés et de fragments d'opaques (pyrite). Au sud du lac Devilliers, mont Bourbeau. Lumière naturelle.

(C) Sédiment mixte polygénétique contenant des fragments de volcanites mafiques de cristallinité variable (à noter la texture microlitique) et de volcanites felsiques, rhyolitiques (à fragments de quartz polycristallins). A 300 mètres au sud-est du lac Devilliers, Formation de Blondeau, mont Bourbeau. Lumière polarisée. 

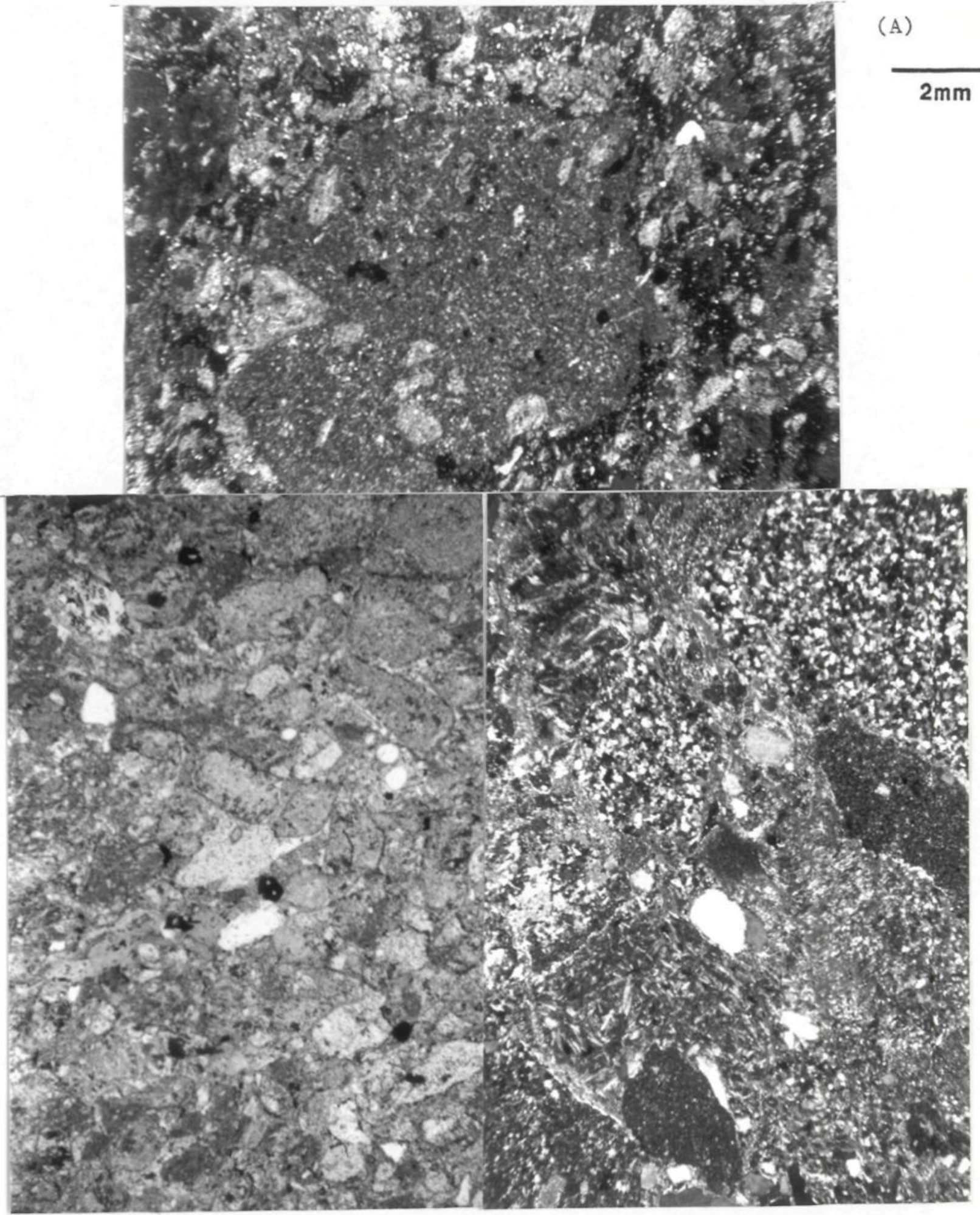

(A)

$2 \mathrm{~mm}$ 
(A) Lentille composée de fragments volcaniques monolithologiques de composition felsique, porphyriques et sub-anguleux. La tête du marteau indique la polarité. Formation de Blondeau, indice du lac Devilliers, mont Bourbeau.

(B) Photomicrographie provenant d'un fragment rhyolitique de la lentille montrée précédemment. Ce fragment contient des phénocristaux altérés de plagioclase, d'amphibole aux bordures chloritisées et des cristaux de quartz bien résorbés. Formation de Blondeau, indice du lac Deviliers. Lumière naturelle.

(C) Même microphotographie que précédemment. La matrice qui contient ces fragments est microcristalline et se compose majoritairement de quartz. Lumière polarisée. 

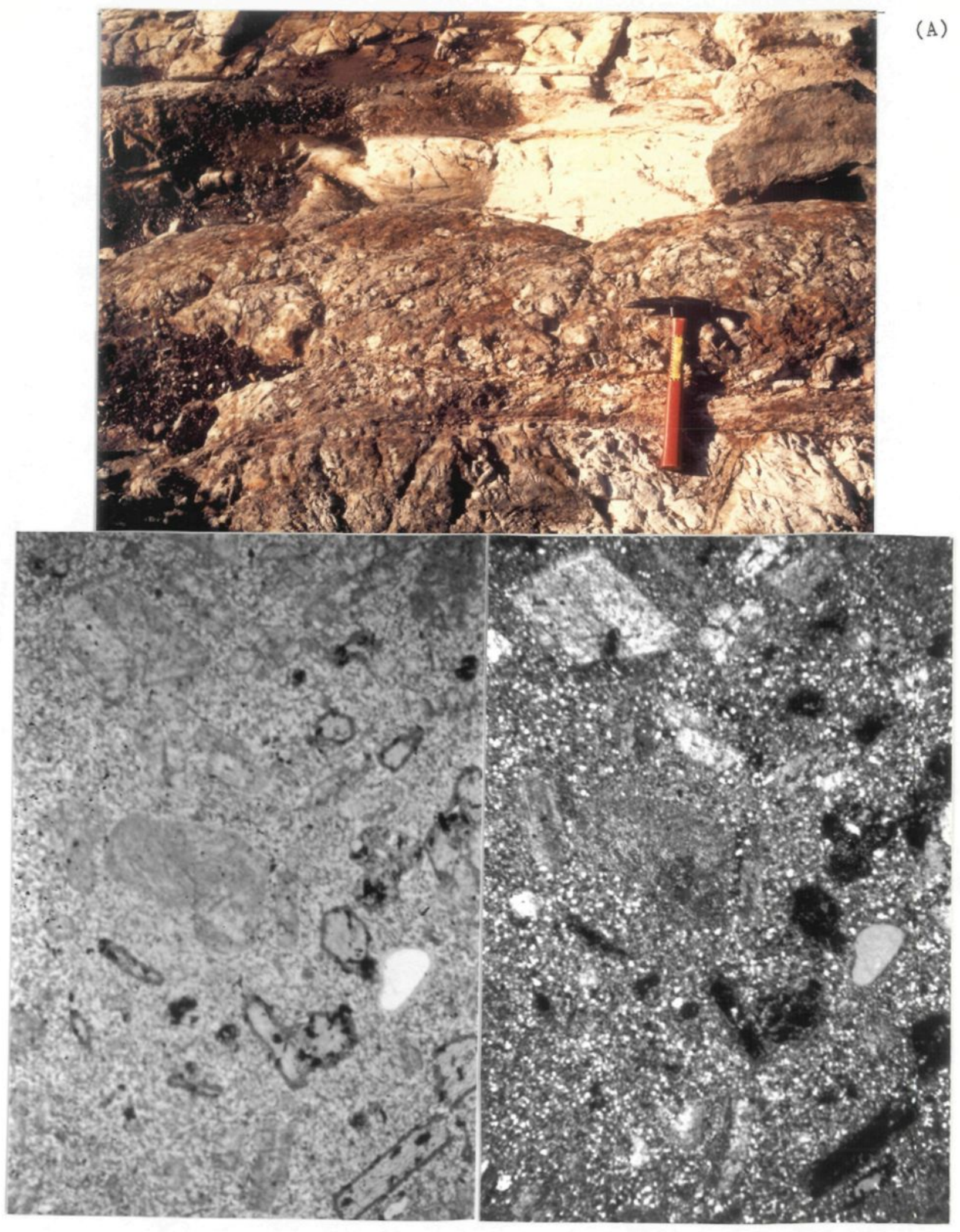

(B)

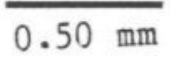

(C)

$\overline{0.50 \mathrm{~mm}}$ 


\section{PLANCHE IV}

(A) Fragment pyriteux, montrant localement des textures colloformes. Ce fragment repose dans une coulée de débris oligolithologique. Formation

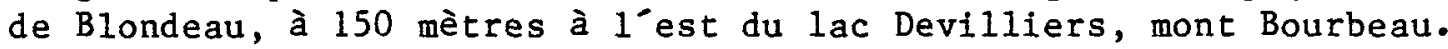

(B) Bombe volcanique (?), de composition felsique et porphyrique. Cette bombe est recouverte successivement par une mince épaisseur de sédiments fins, grèseux à silteux, et par une coulêe de débris. La tête du marteau indique la polarité. Indice du lac Devilliers, mont Bourbeau. 


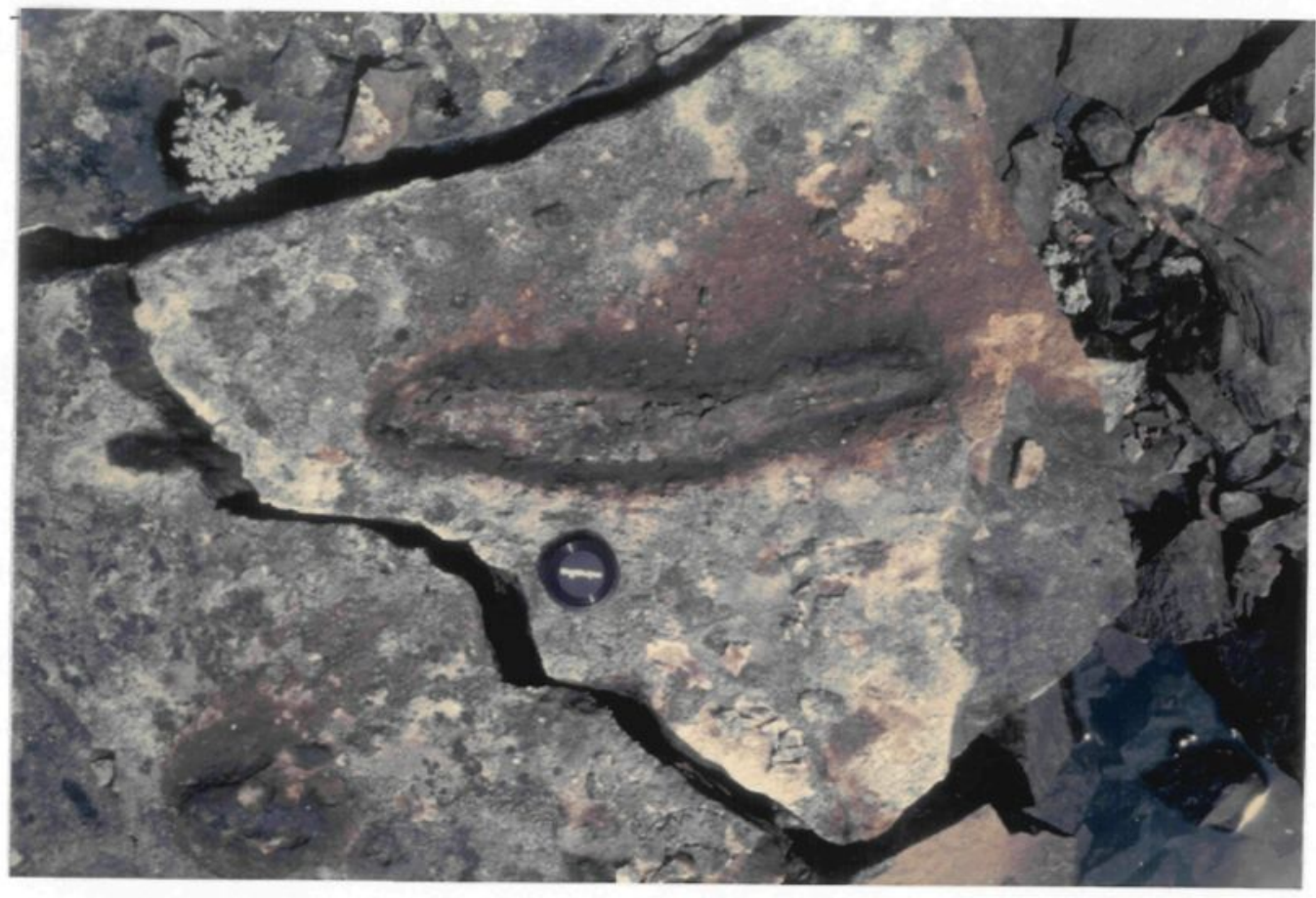

(A)

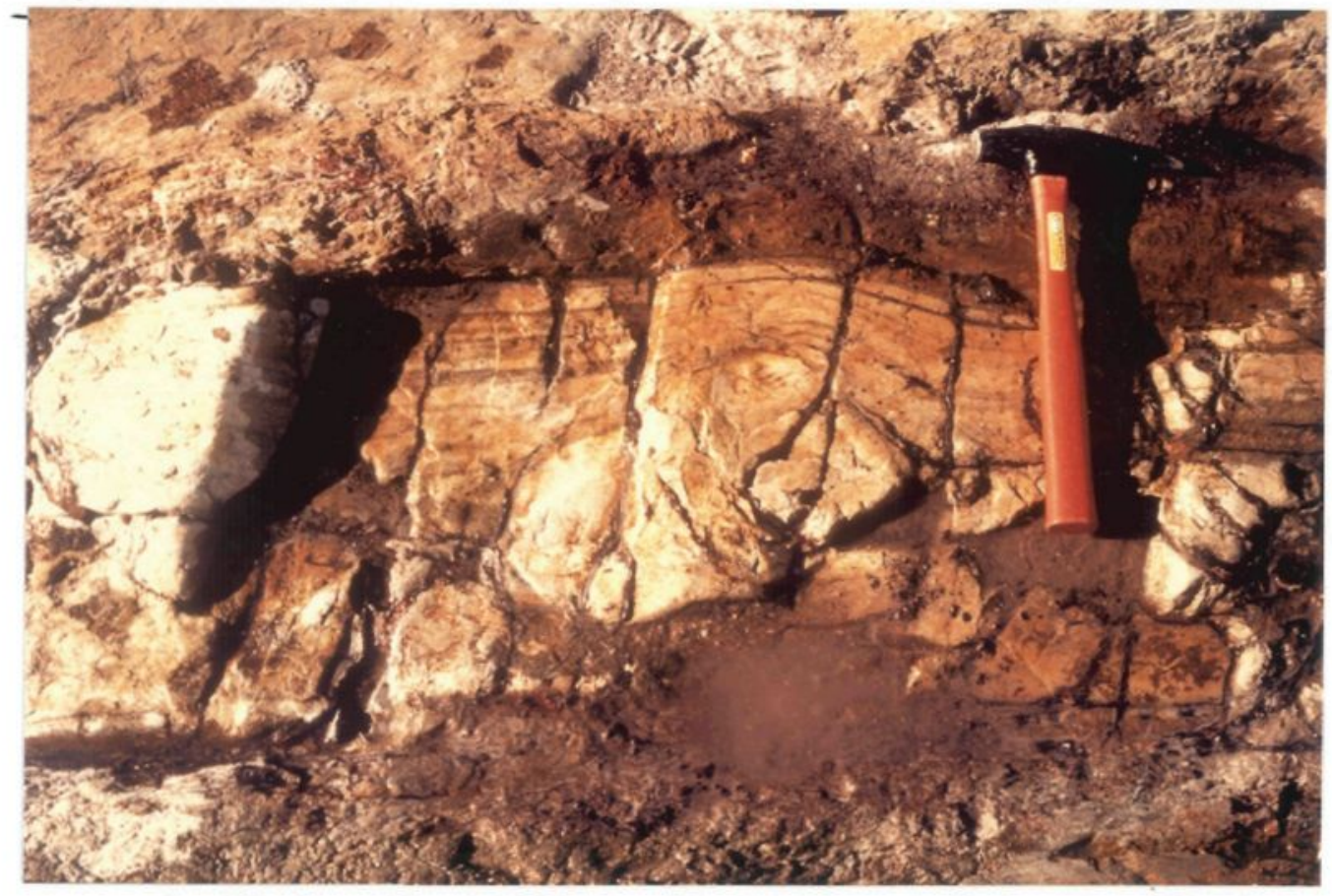

(B) 
PLANCHE V

(A) Sédiments grèseux lités, tronqués par une coulée de débris composée de fragments volcaniques de composition intermédiaire à felsique. A 300 mètres à $1^{\prime}$ est du lac Devilliers, mont Bourbeau.

(B) Base d'une coulée basaltique massive et variolaire. Formation de Blondeau, indice du lac Devilliers, mont Bourbeau. 


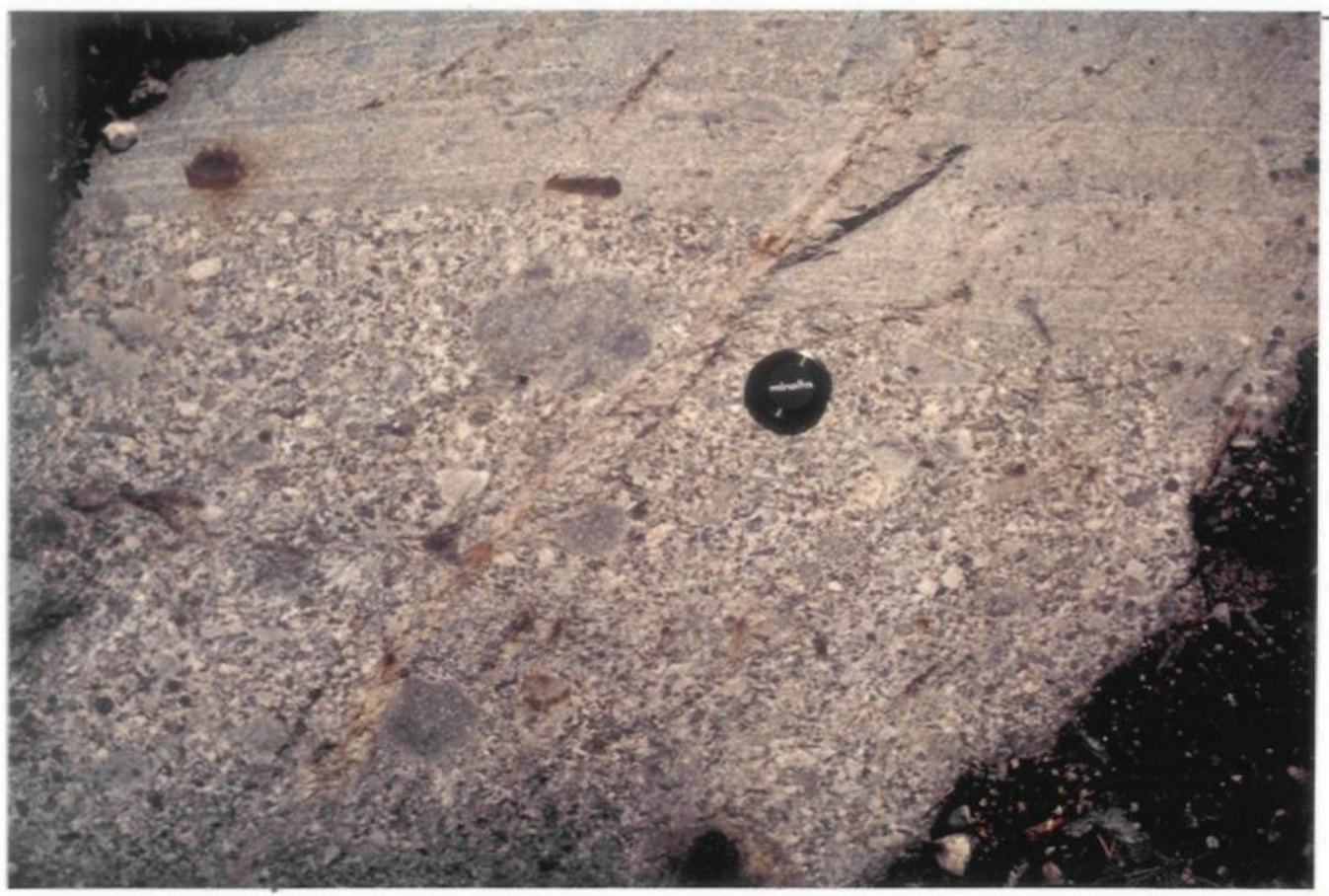

(A)

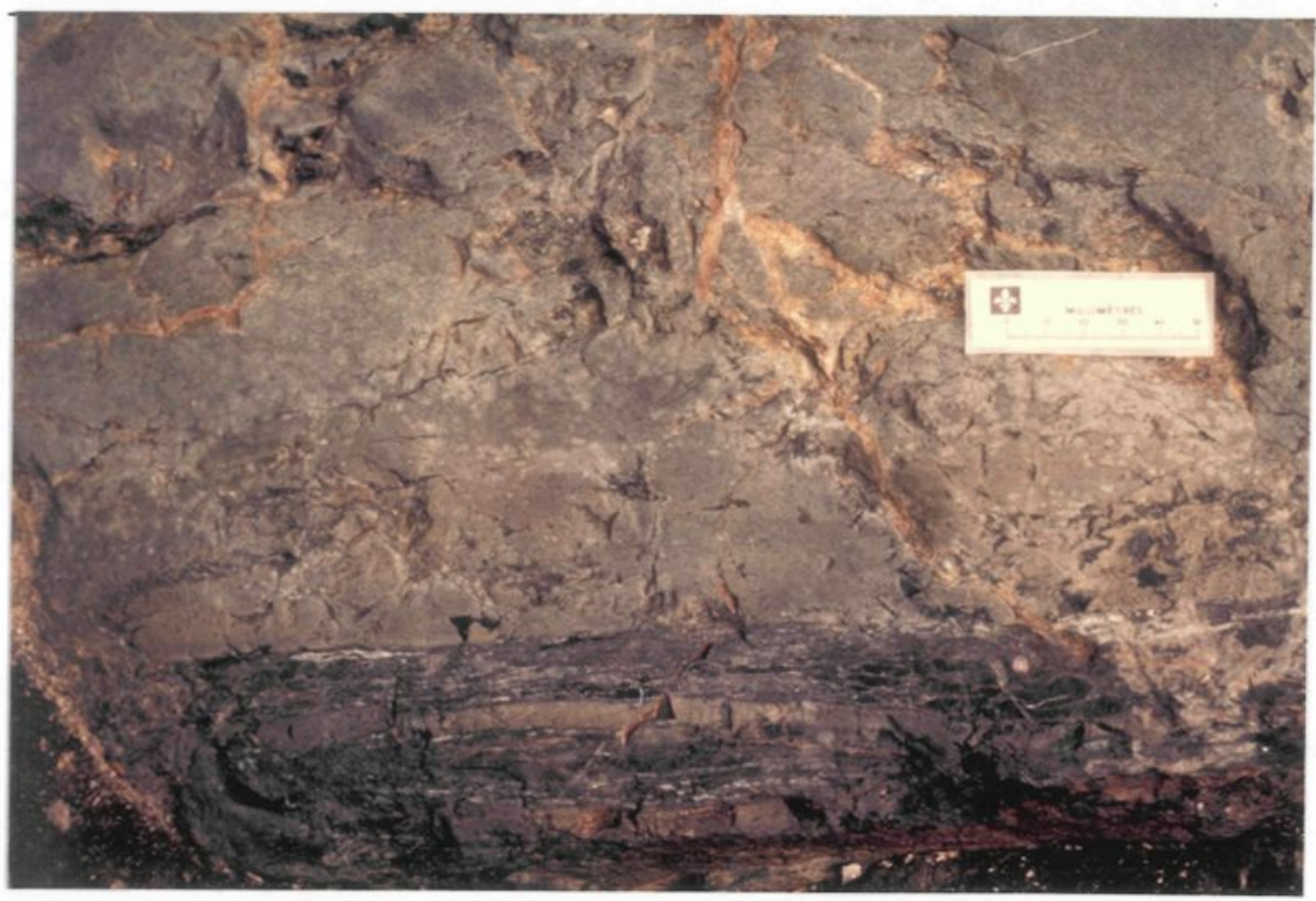

(B) 
PLANCHE VI

(A) Intrusif (?) de composition pyroxénitique, sus-jacent aux laves basaltiques décrites précédemment. La tête du marteau repose sur le basalte.

(B) Basalte intensément bréchifié et localement variolaire. Formation de Blondeau, en bordure de la trace axiale du synclinal de Chibougamau. 


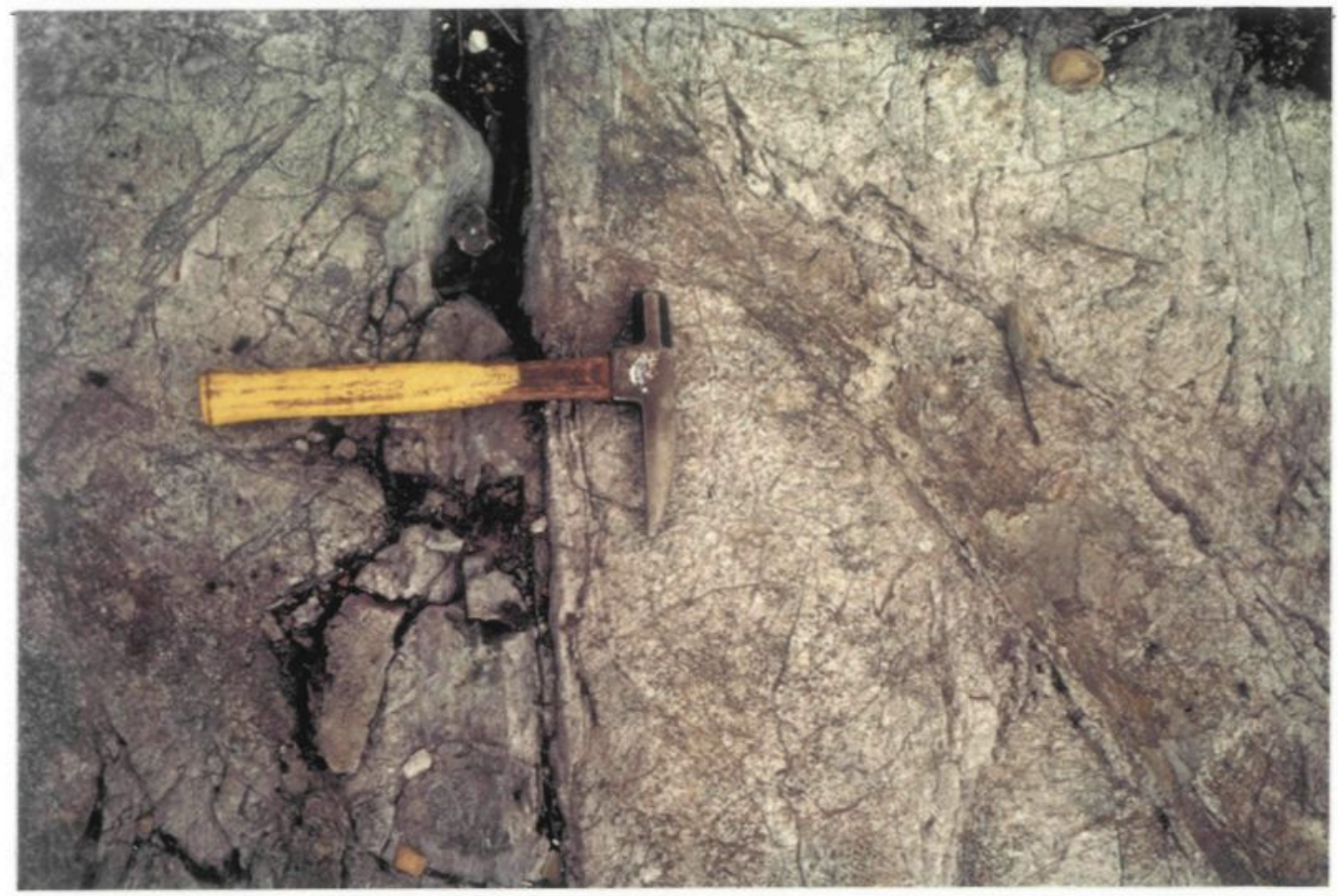

(A)

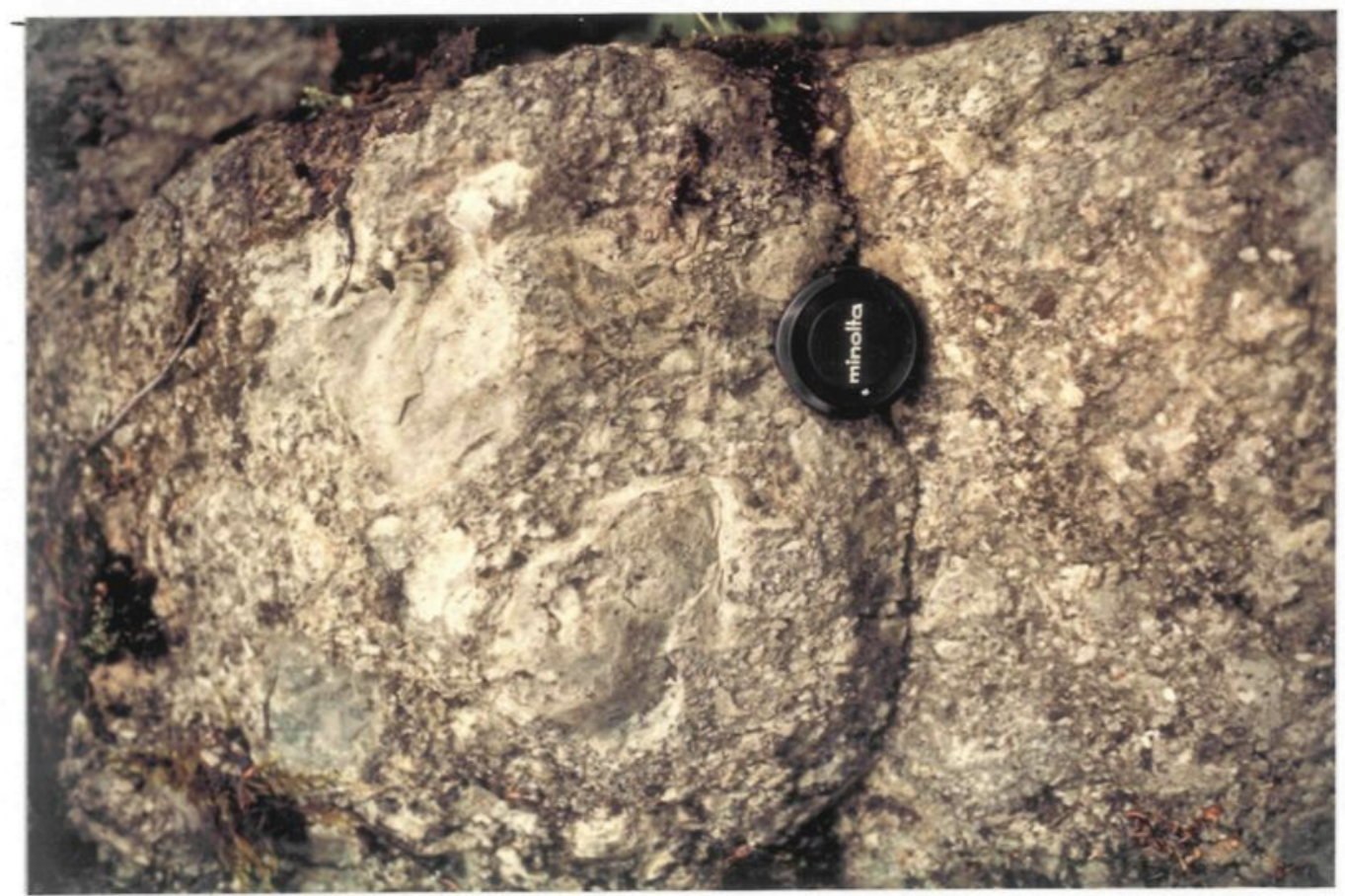

(B) 
PLANCHE VII

(A) Wherlite, composée de cristaux légèrement serpentinisés d'olivine (gris pâle) et de clinopyroxènes ouralitisés fréquemment poecilitiques (noirâtre). Membre inférieur du filon-couche de Roberge, au sud-ouest du lac Larone. Lumière naturelle.

(B) Alternance abrupte entre des bandes de composition dunitique (pâle) composées exclusivement de cristaux ovoĩdes d'olivine, et pyroxénitique (foncée). Membre inférieur du filon-couche de Roberge, au sud du mont Bourbeau. 


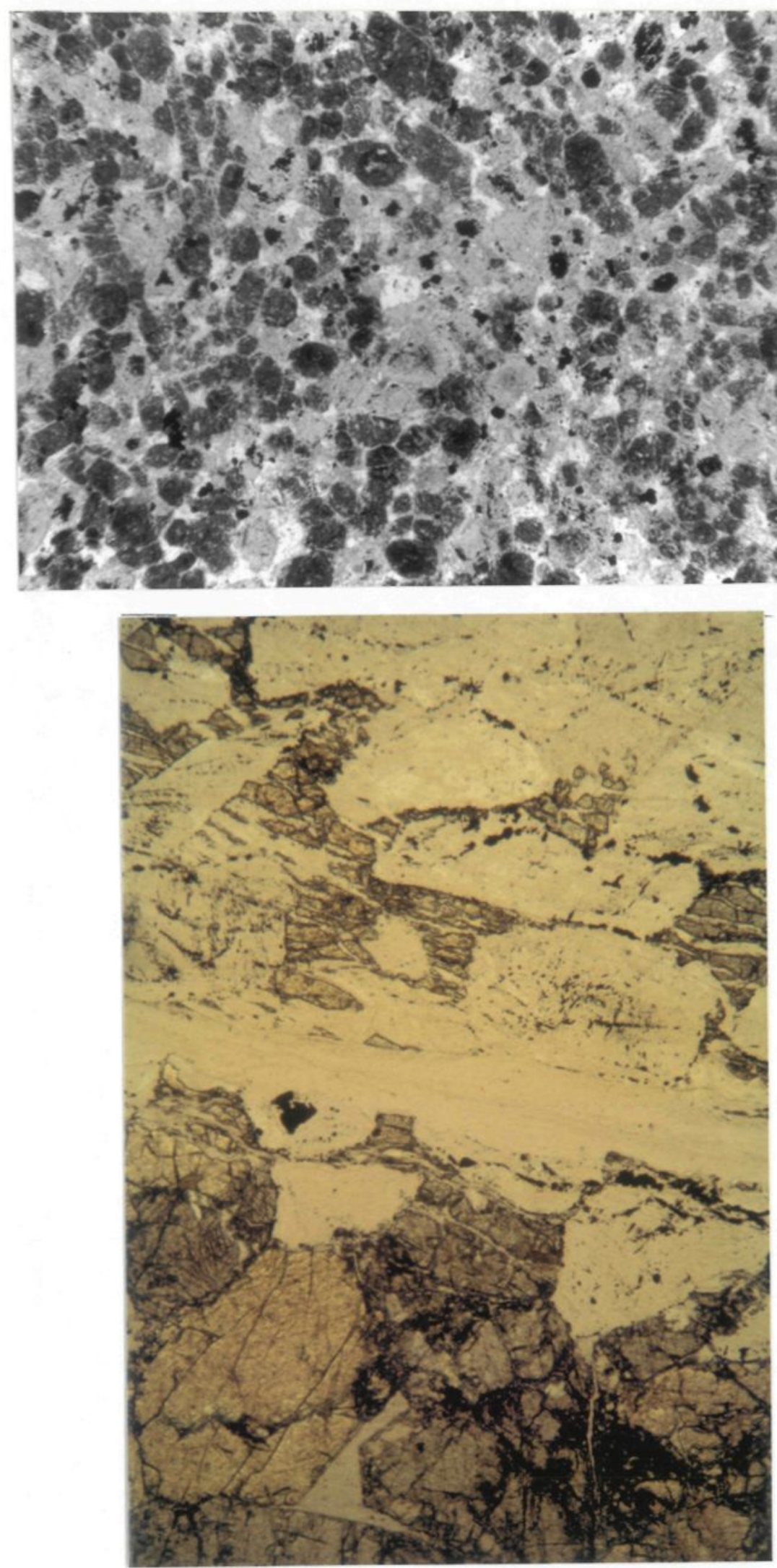

(A)

$2 \mathrm{~mm}$

(B)

$\overline{0.50 \mathrm{~mm}}$ 


\section{PLANCHE VIII}

(A) Bande de composition gabbroĭque parallèle au litage magmatique observé à l'intérieur de la pyroxénite verte du filon-couche de Ventures. Cette pyroxénite est également bien foliée. Mont Bourbeau.

(B) Dyke gabbroìque injecté dans la pyroxénite verte du filon-couche de Ventures. Des minéralisations en pyrite emprunte les bordures et fractures de ce dyke et $s^{\text {”y }}$ retrouvent aussi disséminées. Mont Bourbeau. 


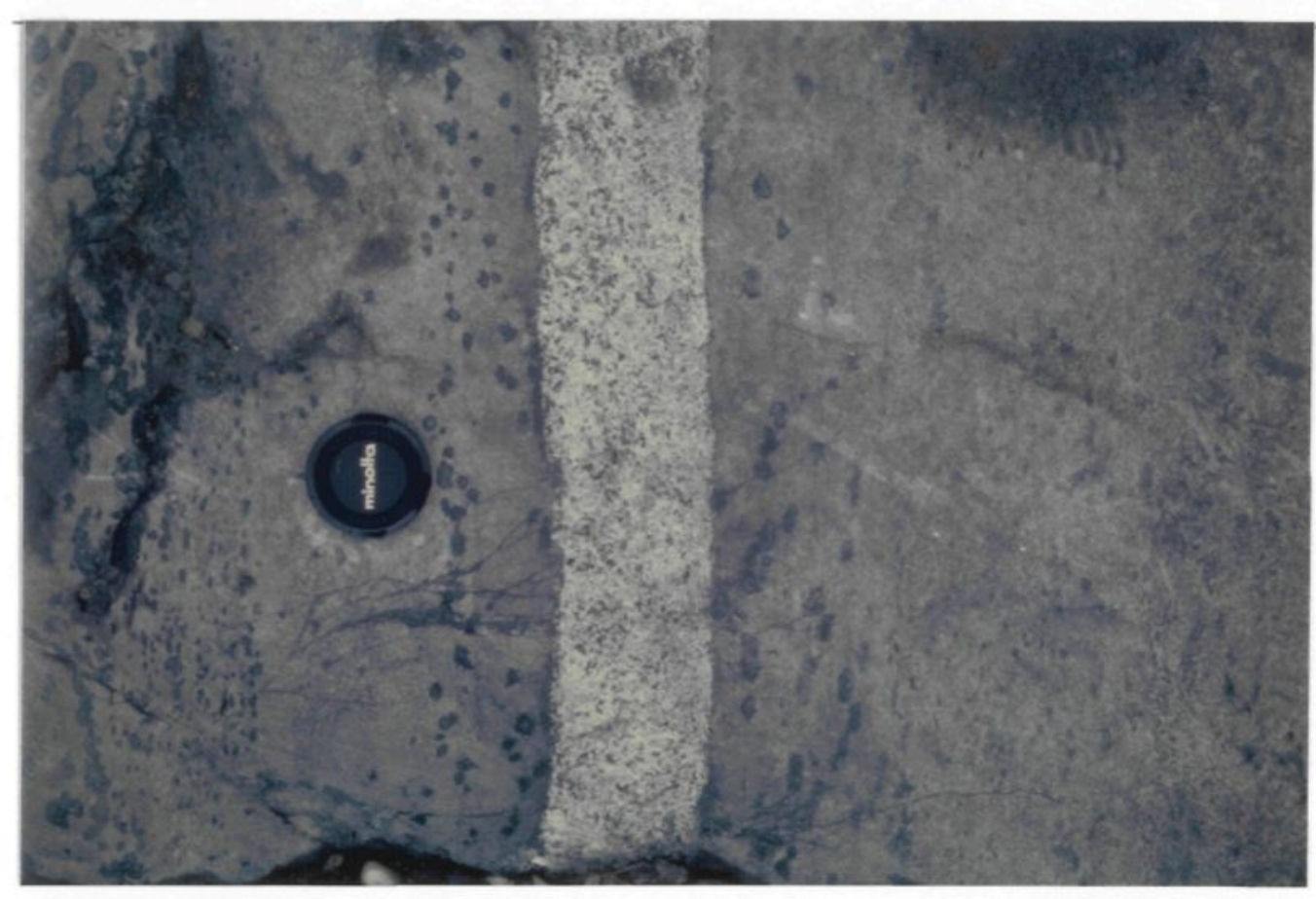

(A)

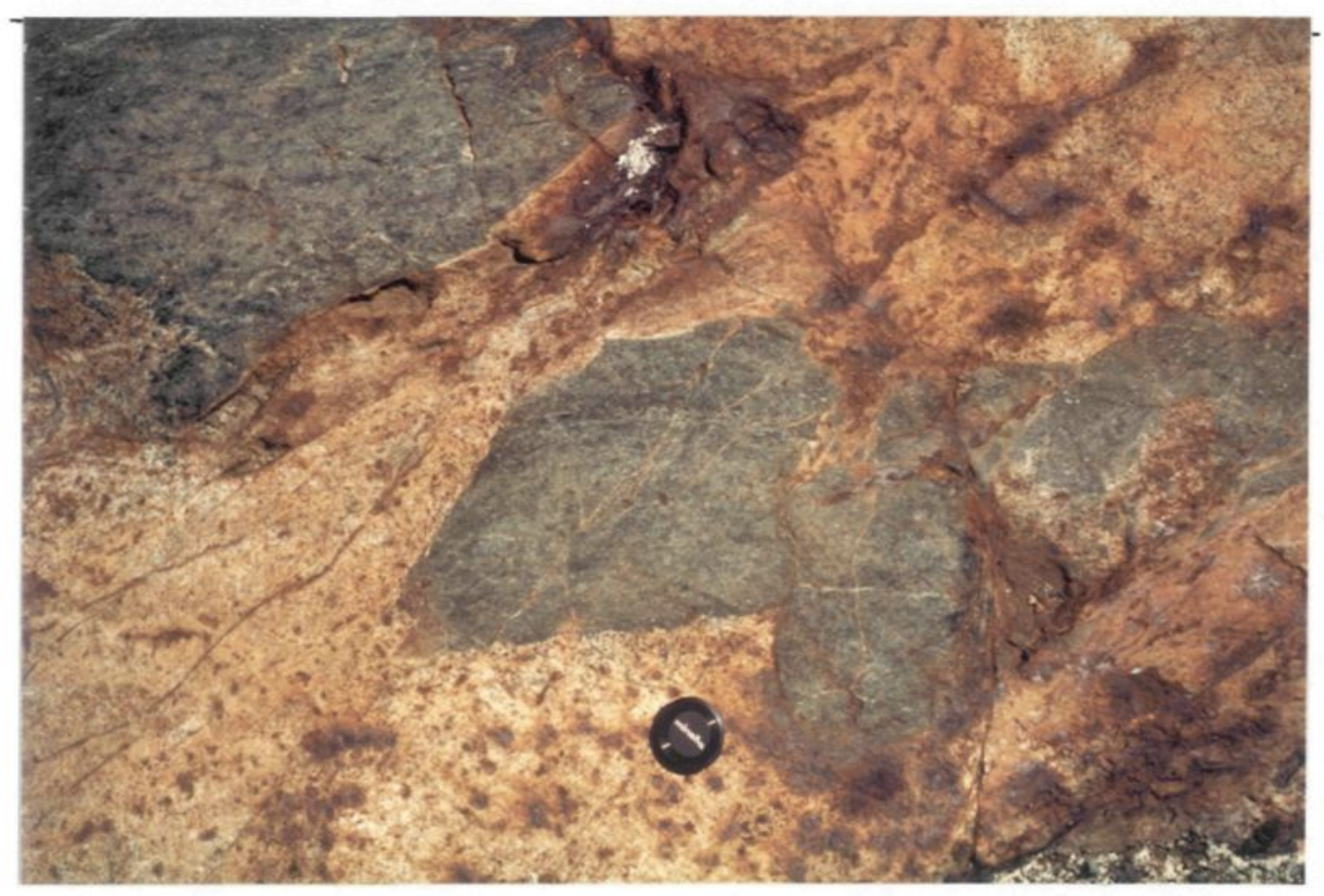

(B) 
PLANCHE IX

(A) Pyroxénite gabbrol̆que foliée, provenant de la zone de transition entre la pyroxénite et le gabbro. Les clinopyroxènes sont ouralitisés. Filon-couche de Ventures, mont Bourbeau. Lumière naturelle.

(B) Contact basal entre la bordure de trempe du filon-couche de Bourbeau (sous la tête du marteau) et les sédiments grèseux et lités de la Formation de Blondeau. A 300 mètres à 1'est du lac Devilliers, mont Bourbeau.

(C) Sédiment grèseux, composé de cristaux libres de plagioclase légèrement séricitisés et de quelques cristaux de quartz. Ce sédiment est plus ou moins silicifié et chloritisé. Formation de Blondeau, à la base du filon-couche de Bourbeau, mont Bourbeau. Nicols croisés. 


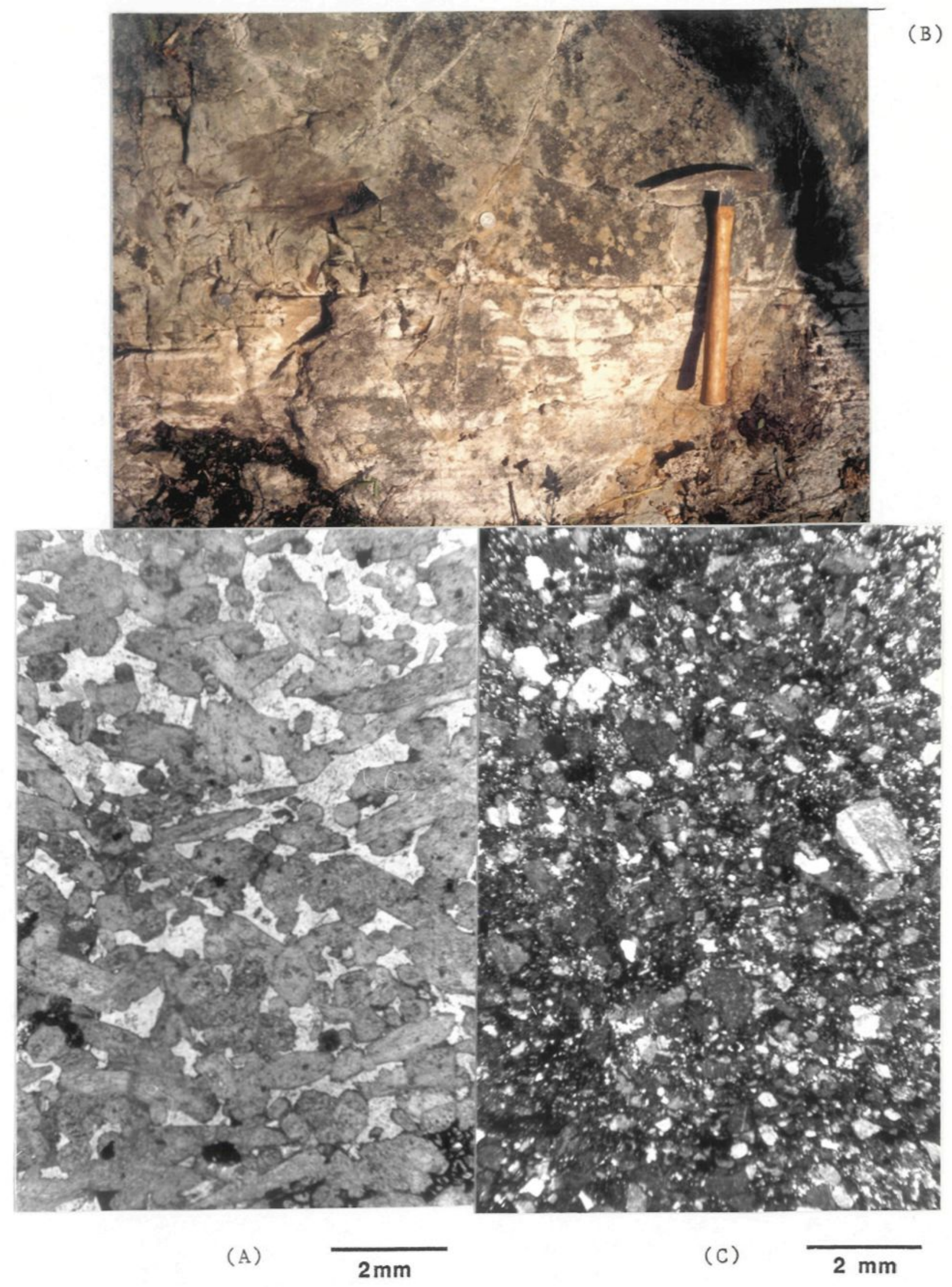


PLANCHE X

(A) Microphotographie du membre dunitique basal du filon-couche de Bourbeau, composé de cristaux ovol̀des d'olivine (pâle) et de cristaux poecilitiques d'orthopyroxène (bronzite ?). A 400 mètres à $1^{\prime}$ est du lac Devilliers, mont Bourbeau. Lumière naturelle.

(B) Lentille "pegmatitique" de composition gabbrolque prenant place dans le ferrogabbro du filon-couche de Bourbeau. Au NW du lac Larone. 


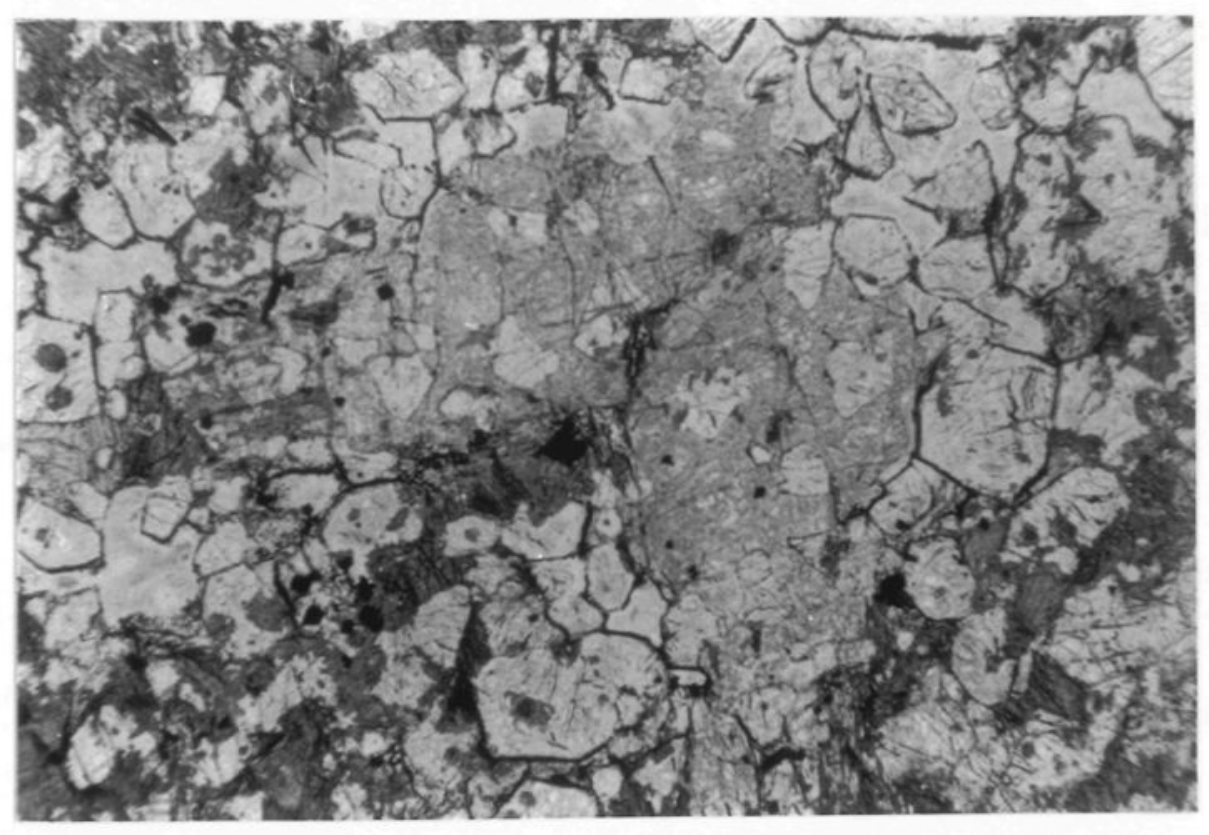

(A)

\section{$2 \mathrm{~mm}$}

(B)

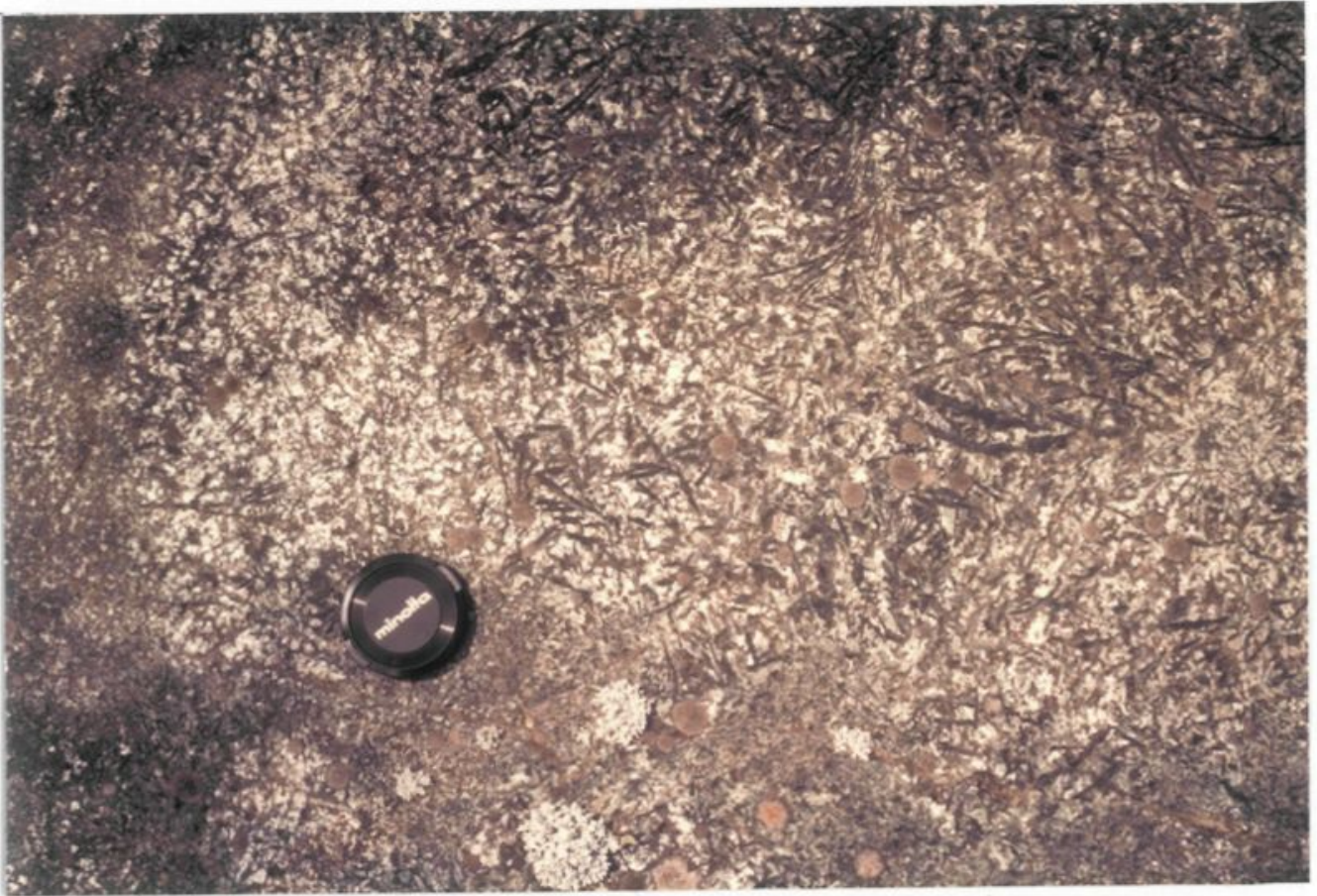


PLANCHE XI

(A) Microphotographie d'un dyke à phénocristaux de plagioclase peu altérés et de quartz. La matrice se compose pour la plus grande part de quartz microcristallin. Indice des Dômes du radar, mont Bourbeau. Nicols croisés.

(B) Microphotographie d'un dyke porphyrique en pyroxènes, totalement ouralitisés. La matrice consiste en un assemblage d'aiguilles d'amphibole (actinote), de chlorite et de rares feldspaths. Formation de Haüy (?), à $1^{\text {-ouest }}$ du lac Ham. Lumière naturelle. 

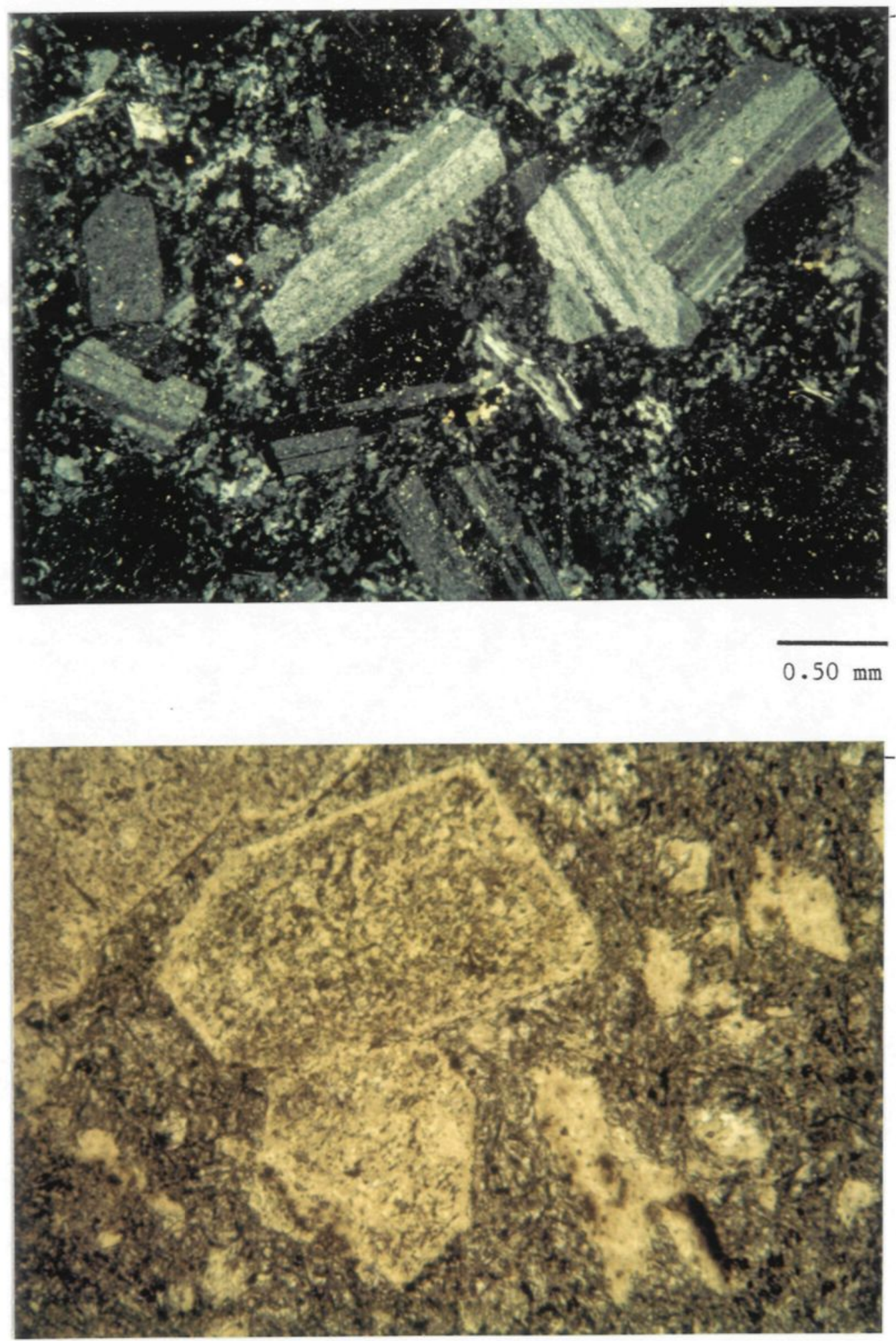

(B) 
PLANCHE XII

Leucotonalite massive (L) bordée dans sa partie supérieure par une syénite porphyrique en amphibole $(S)$. Une veine composée $d^{\prime}$ axinite (rosée) et localement d'épidote (vert) recoupe ces deux lithologies. Bloc rocheux provenant de la galerie d'exploration de 1"indice "Be1-3", mont Bourbeau. 


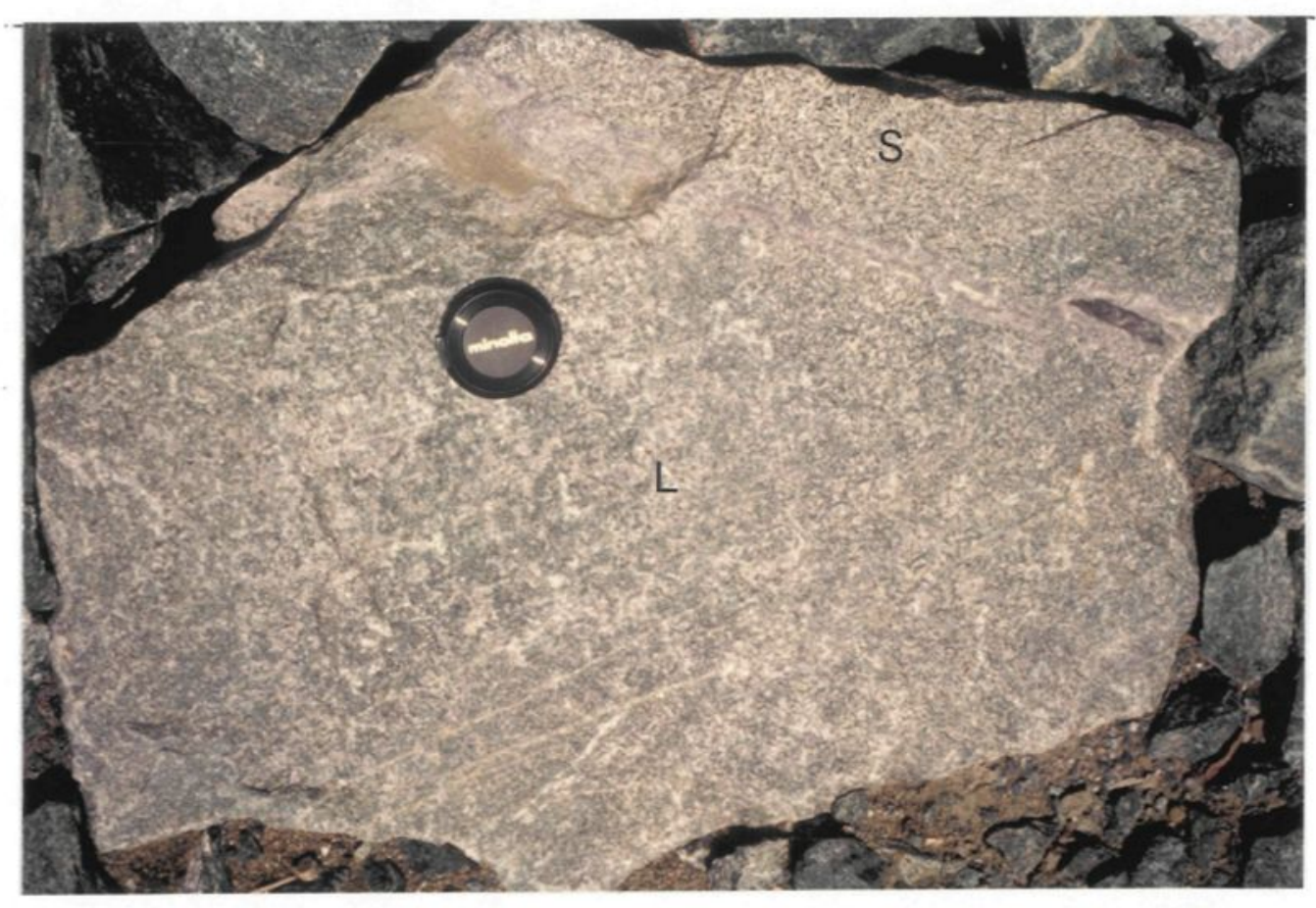


PLANCHE XIII

(A) Leucotonalite, lumière naturelle. Cette roche se compose de phénocristaux de plagioclase, séricitisés de façon variable, d’amphibole et de quartz. Stock tonalitique du lac Line, échantillon recueilli au sud de la Baie du Cran Penché.

(B) Leucotonalite, nicols croisés. L'altération des phénocristaux de plagioclase et $d^{-}$amphibole est peu intense. La matrice consiste quasi exclusivement de cristaux de quartz microcristaliin et de rares feldspaths. 


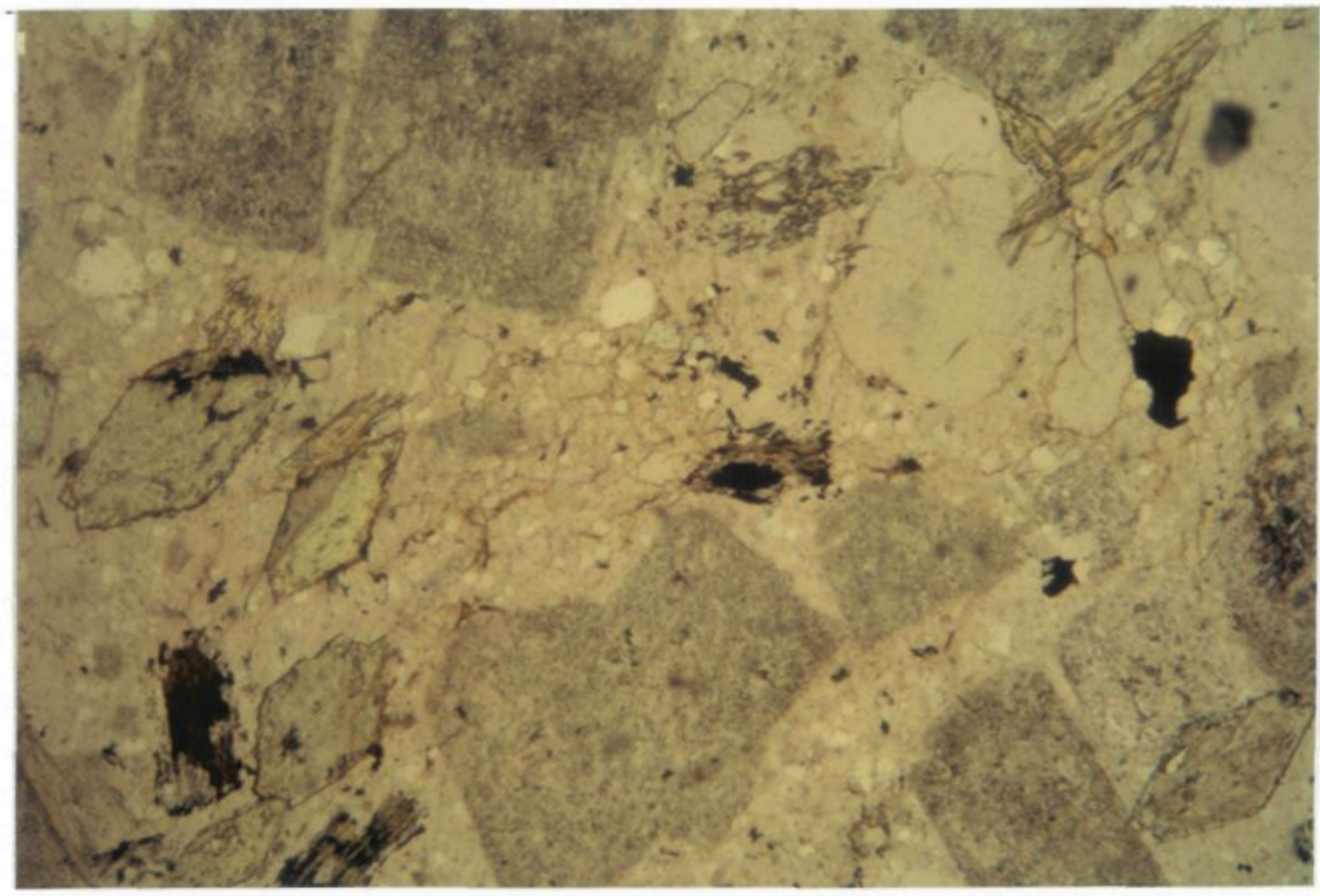

(A)
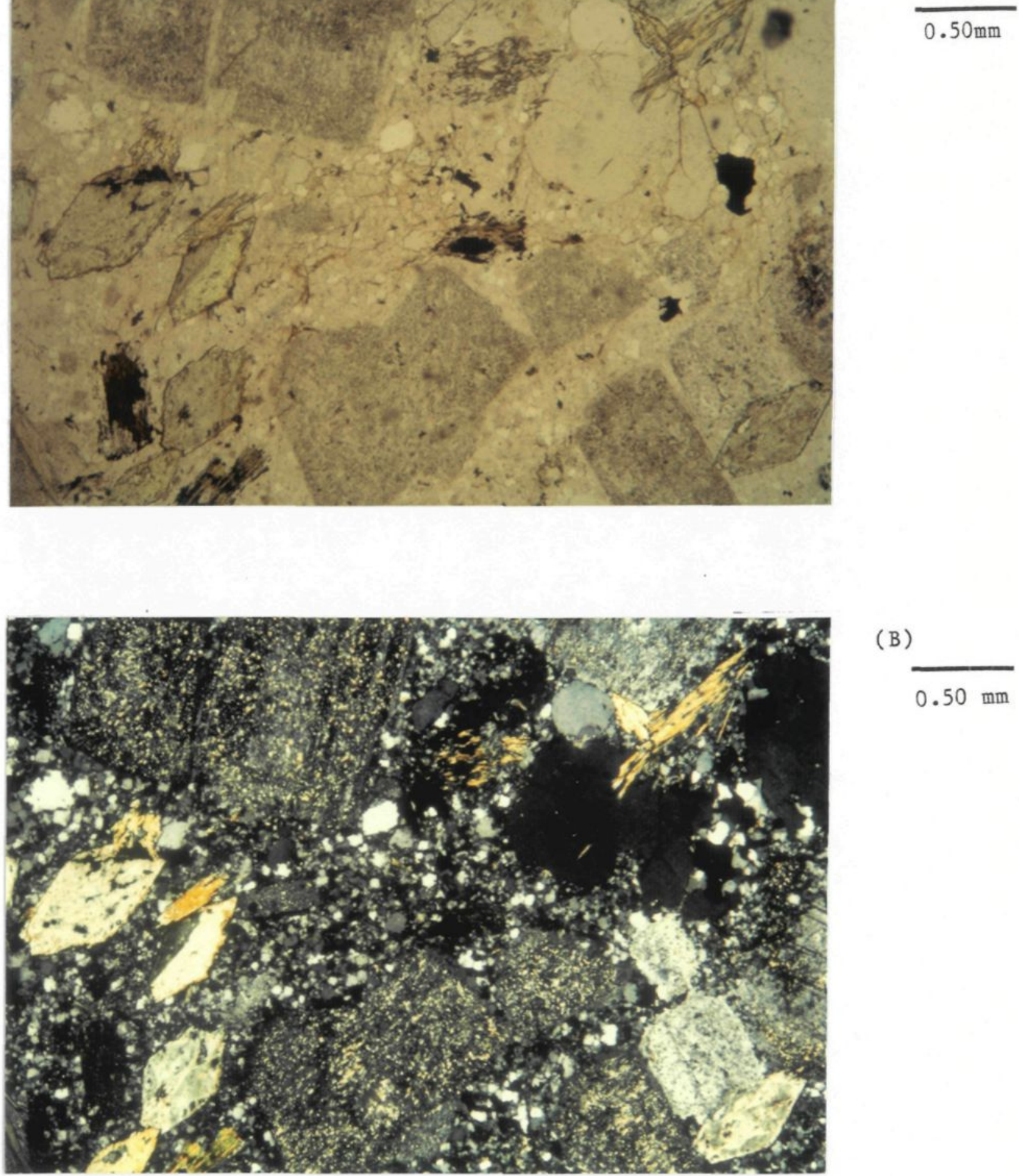

(B)

$0.50 \mathrm{~mm}$ 
PLANCHE XIV

(A) Microphotographie d'une syénite porphyrique en amphibole (foncé) et en feldspath (orthose) légèrement séricitisés. Echantilion provenant de la galerie d'exploration de 1'indice "Be1-3", mont Bourbeau. Lumière naturelle.

(B) Faciès dioritique de bordure du stock du lac line (gris noirâtre) en contact graduel avec une leucotonalite (sous le capuchon). Cette diorite est souvent foliée parallèlement au contact avec les volcanites encaissantes. Au nord-ouest du lac Ham.

(C) Faciès de cornéenne résultant du métamorphisme de contact amené par le stock tonalitique du lac line et qui est observé dans des sédiments grèseux épiclastiques et lités de la Formation de Blondeau. Cet affleurement se trouve à moins de 100 mètres de la bordure de ce stock et à environ 400 mètres au sud-est du lac Line, mont Bourbeau. 


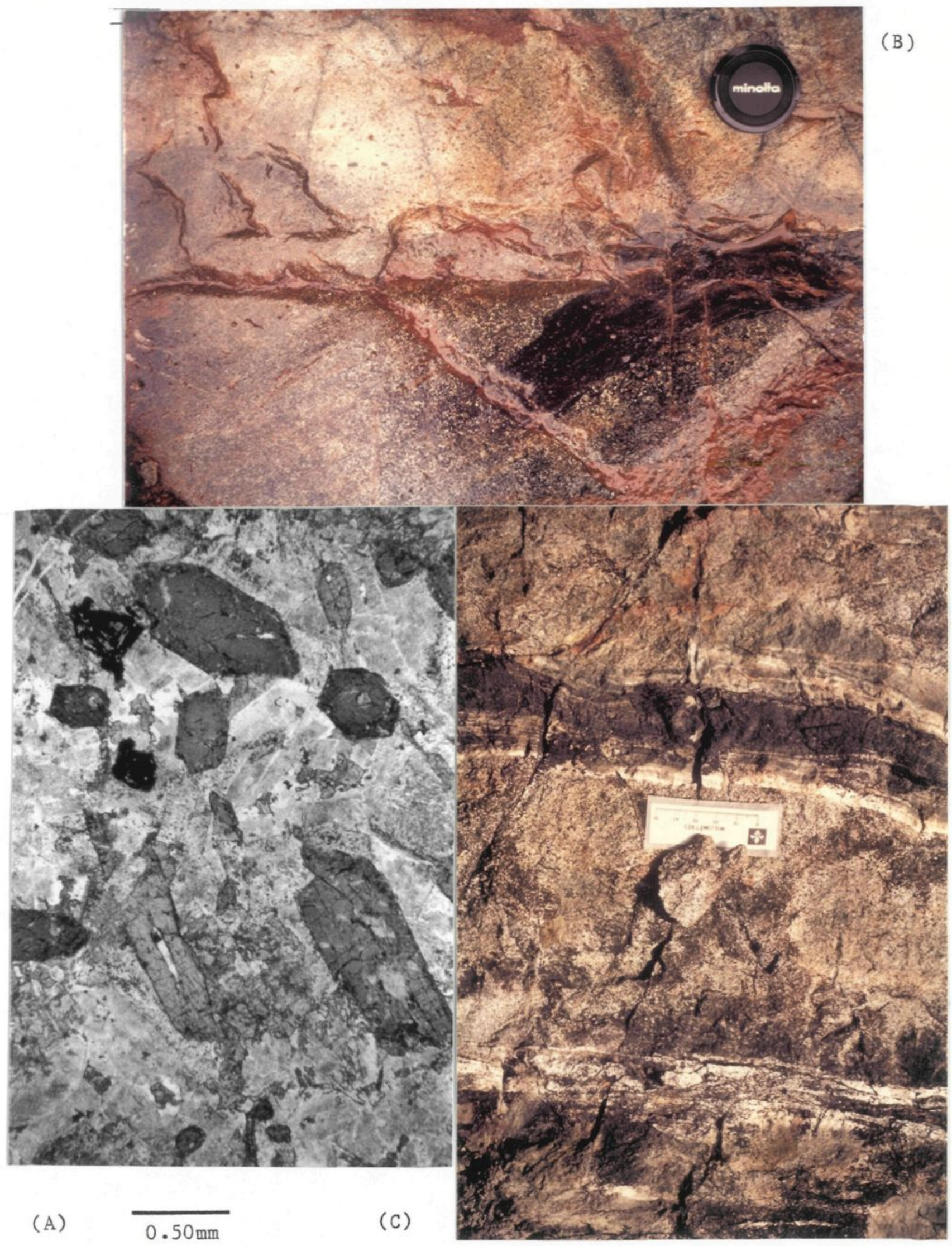


PLANCHE XV

Pli d'entrainement serré observé dans une péridotite serpentinisée, membre supérieure du filon-couche de Roberge. Le nord est du côté gauche. Ce pli, un anticlinal, possède une charnière inclinée vers $1^{\text {'est et }}$ il se situe en bordure nord d'une faille inverse majeure, d'orientation E-W. Ces caractéristiques signifient que la composante horizontale du mouvement global le long de cette faille est dextre. Au nord des indices des Dômes du radar, mont Bourbeau. 


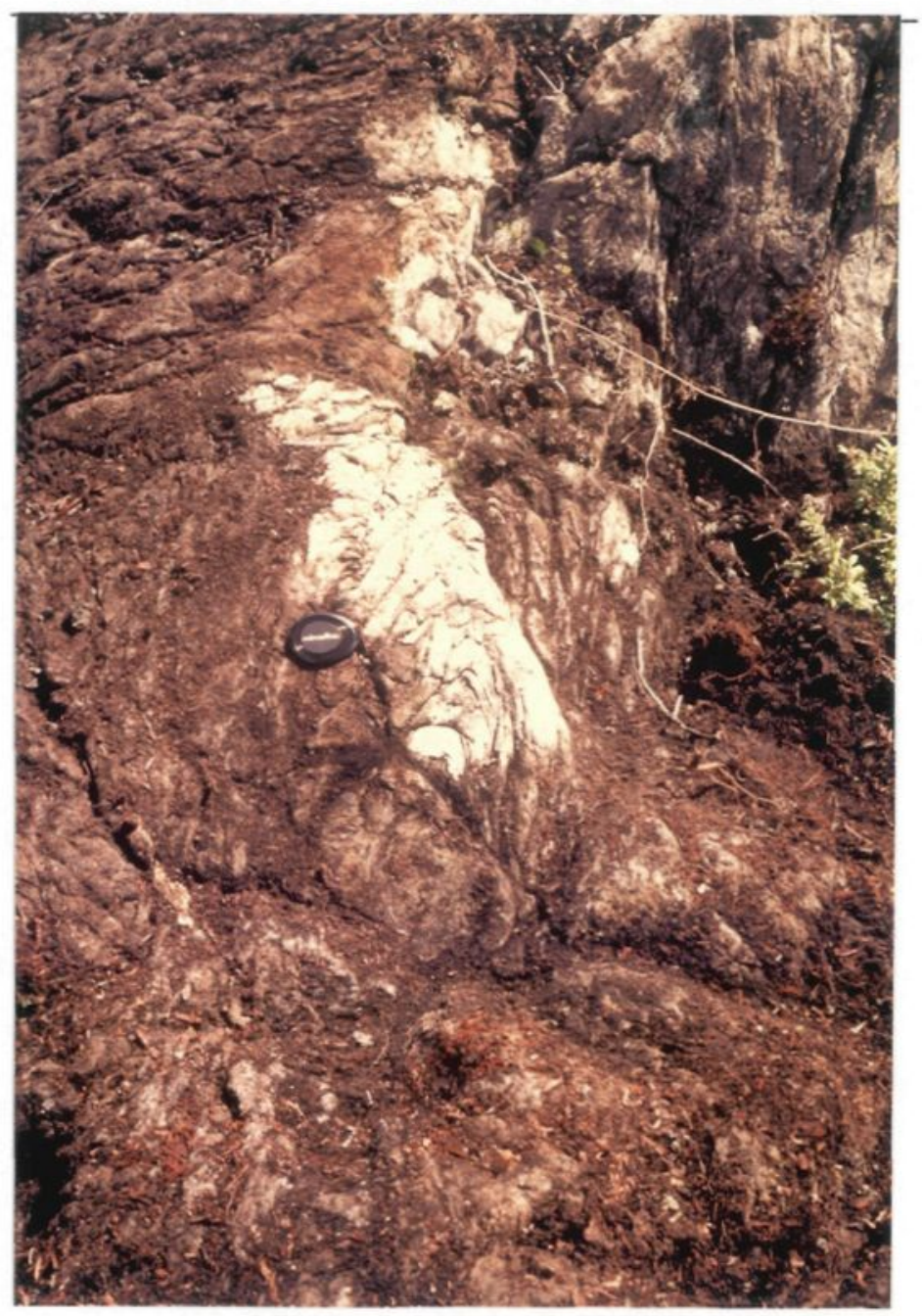


PLANCHE XVI

(A) Fragments basaltiques silicifiés, contenant des microlites de plagioclase. La matrice est constituée de fragments plus petits de chlorite, de quartz et de carbonate. Formation de Gilman, indice du lac Ham. Lumière naturelle.

(B) Filonets riches en pyrite et en chalcopyrite qui bréchifient une minéralisation antérieure composée de magnétite massive. Indice du lac Ham.

(C) Injection et comblement par la chalcopyrite (jaune foncé) de fractures dans des grains de pyrite recristallisés en porphyroblastes (jaune pâle) et de magnétite. Indice du lac Ham. Lumière réfléchie. 


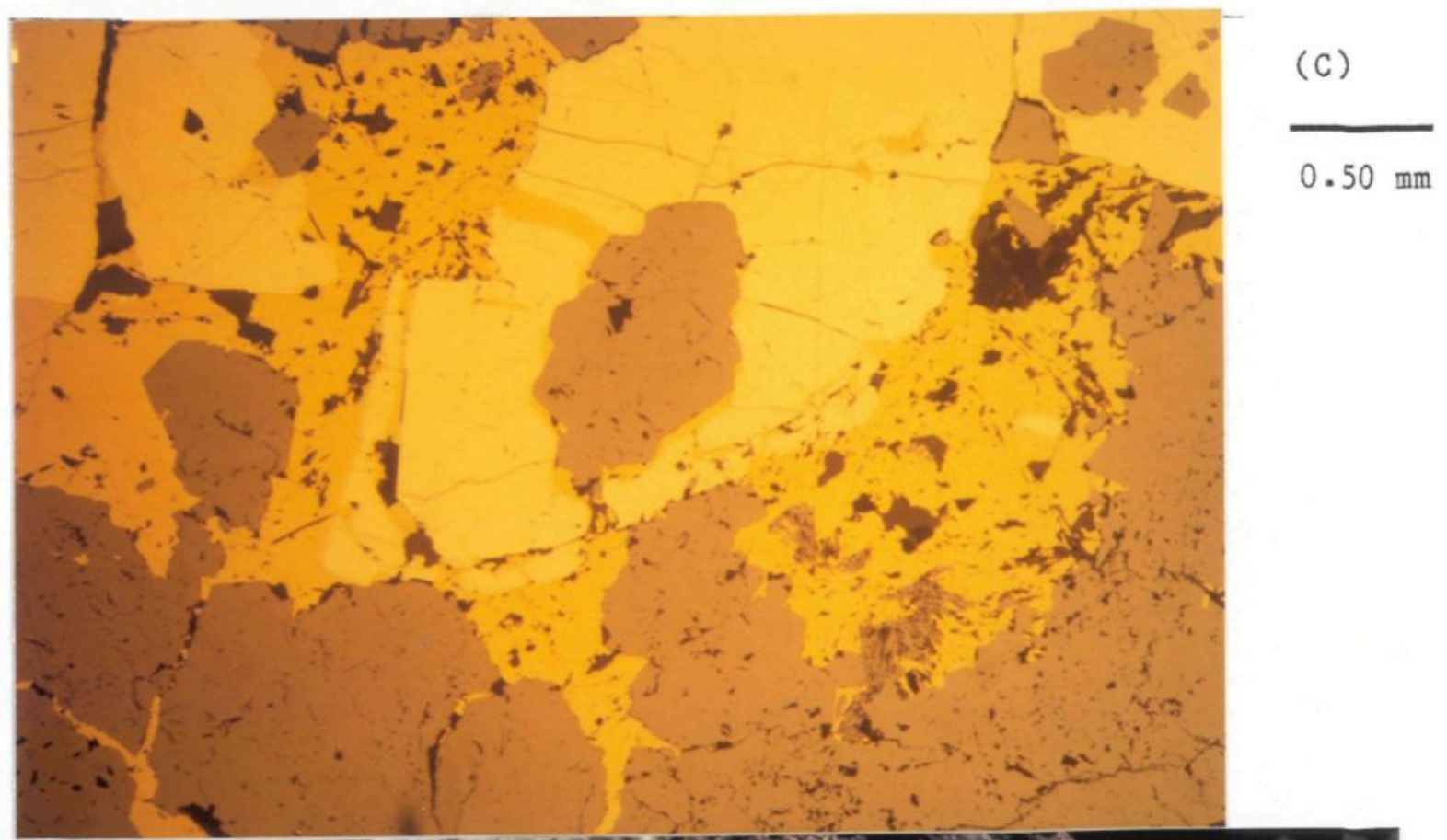

H.

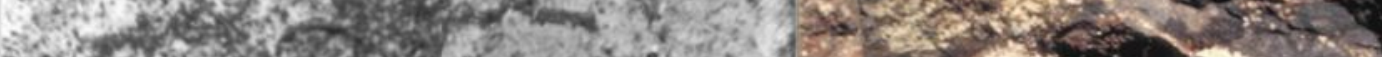

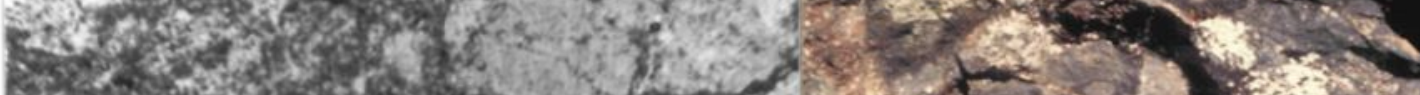

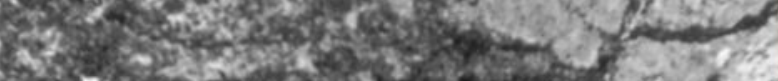

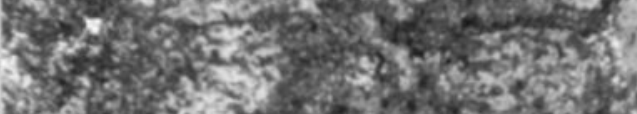

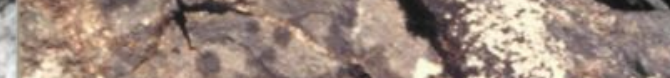

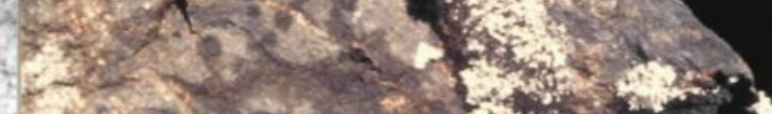

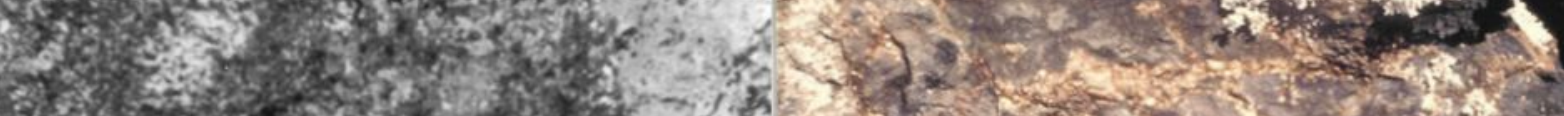

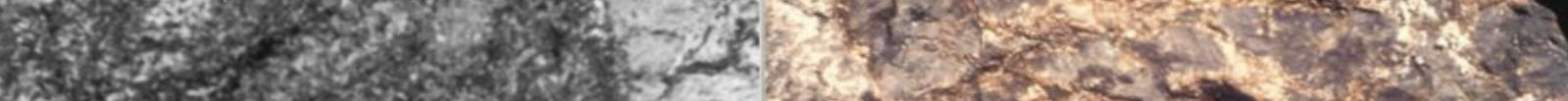

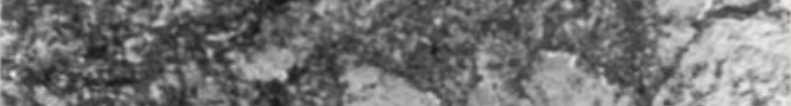

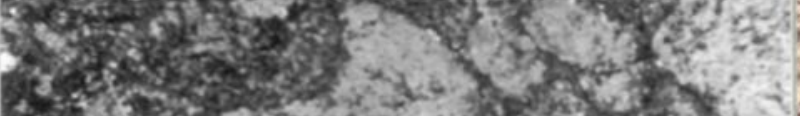

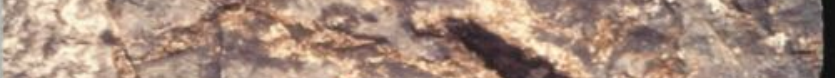

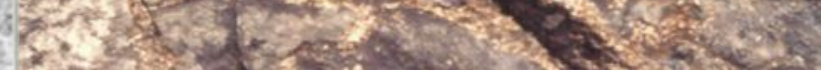

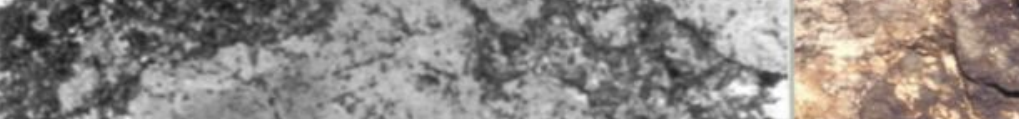

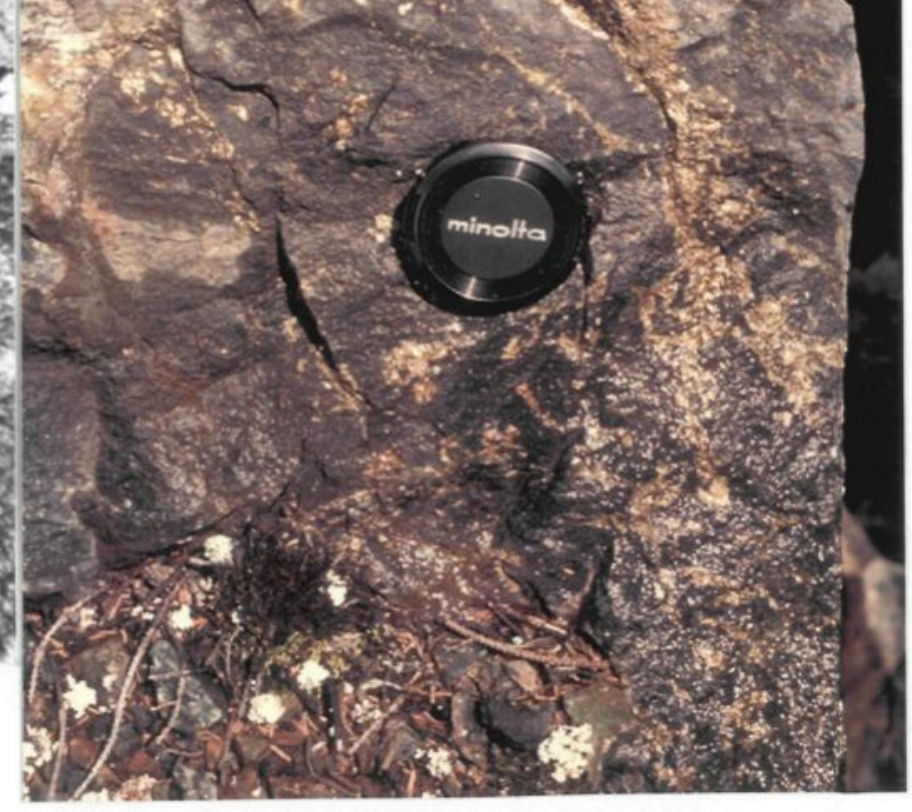

(A)

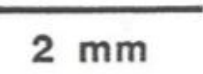


PLANCHE XVII

(A) Lentilles pyriteuses stratiformes reposant sur un sédiment épiclastique. La tête du marteau donne la polarité. Formation de Blondeau, au sud du lac Larone.

(B) Détails de la photographie précédente. Les lits minéralisés sont très minces et ne contiennent que de la pyrite. 


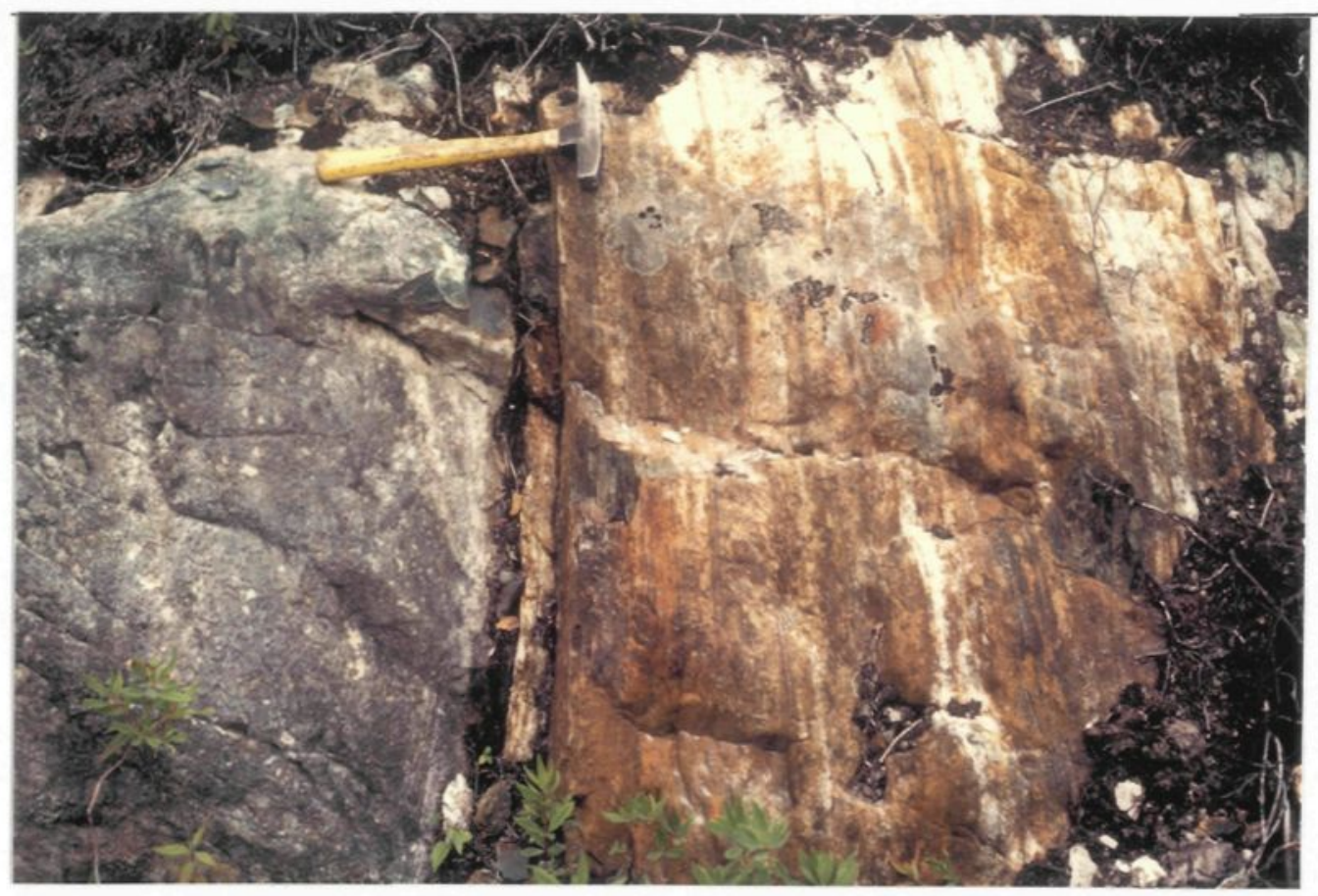

(A)

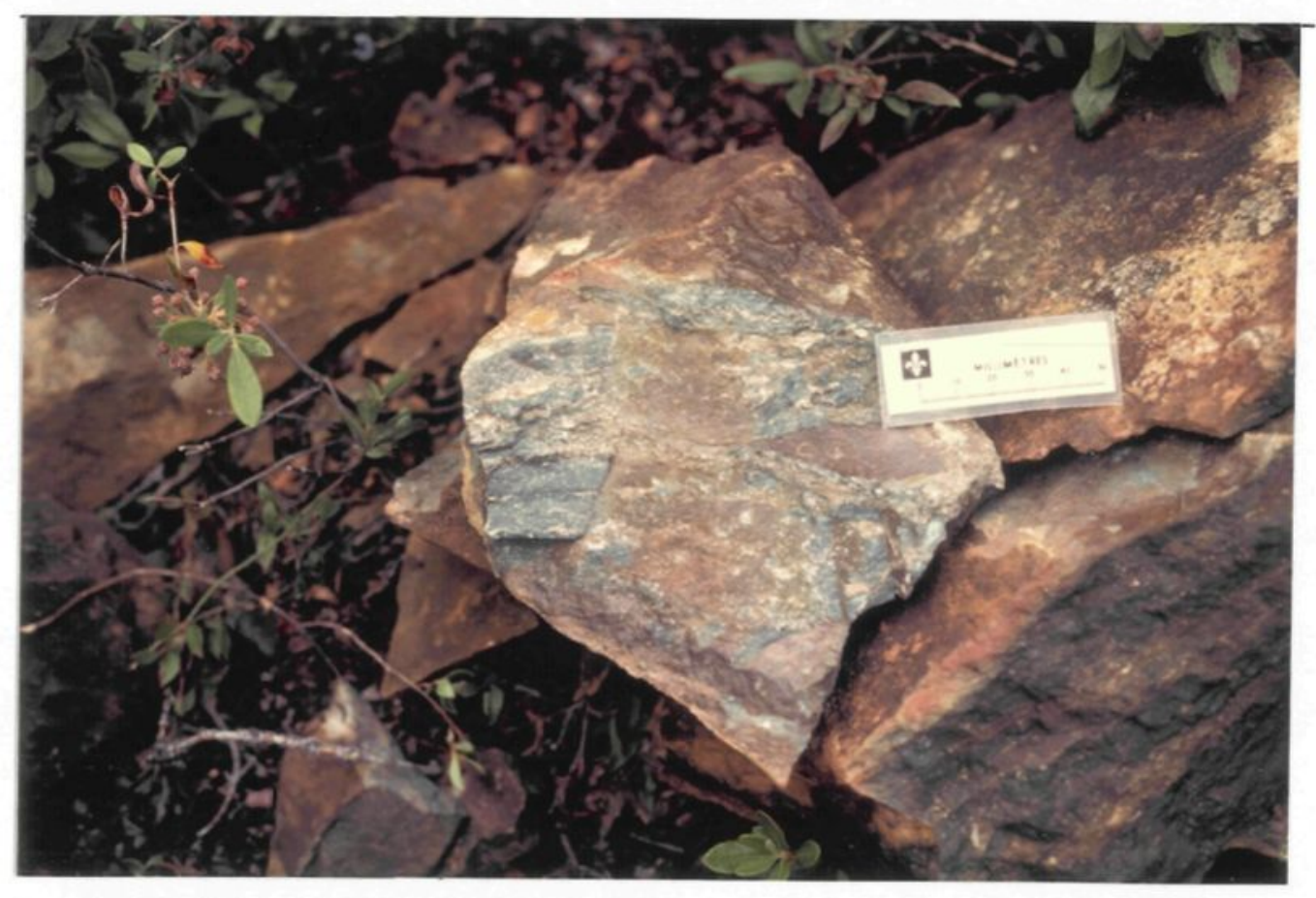


PLANCHE XVIII

\begin{abstract}
(A) Veinules discordantes minéralisées en pyrrhotine et plus ou moins en pyrite. Les sédiments épiclastiques encaissants sont fortement carbonatisés, chloritisés et silicifiés. La tête du marteau souligne la direction générale de la stratification et donne la polarité. Formation de Blondeau, indice du lac Devilliers.
\end{abstract}

(B) Fragment minéralisé plissé similaire aux minéralisations stratiformes stratigraphiquement sousjacentes. Ce fragment est incorporé à une coulée basaltique. Formation de Blondeau, indice du lac Devilliers. 


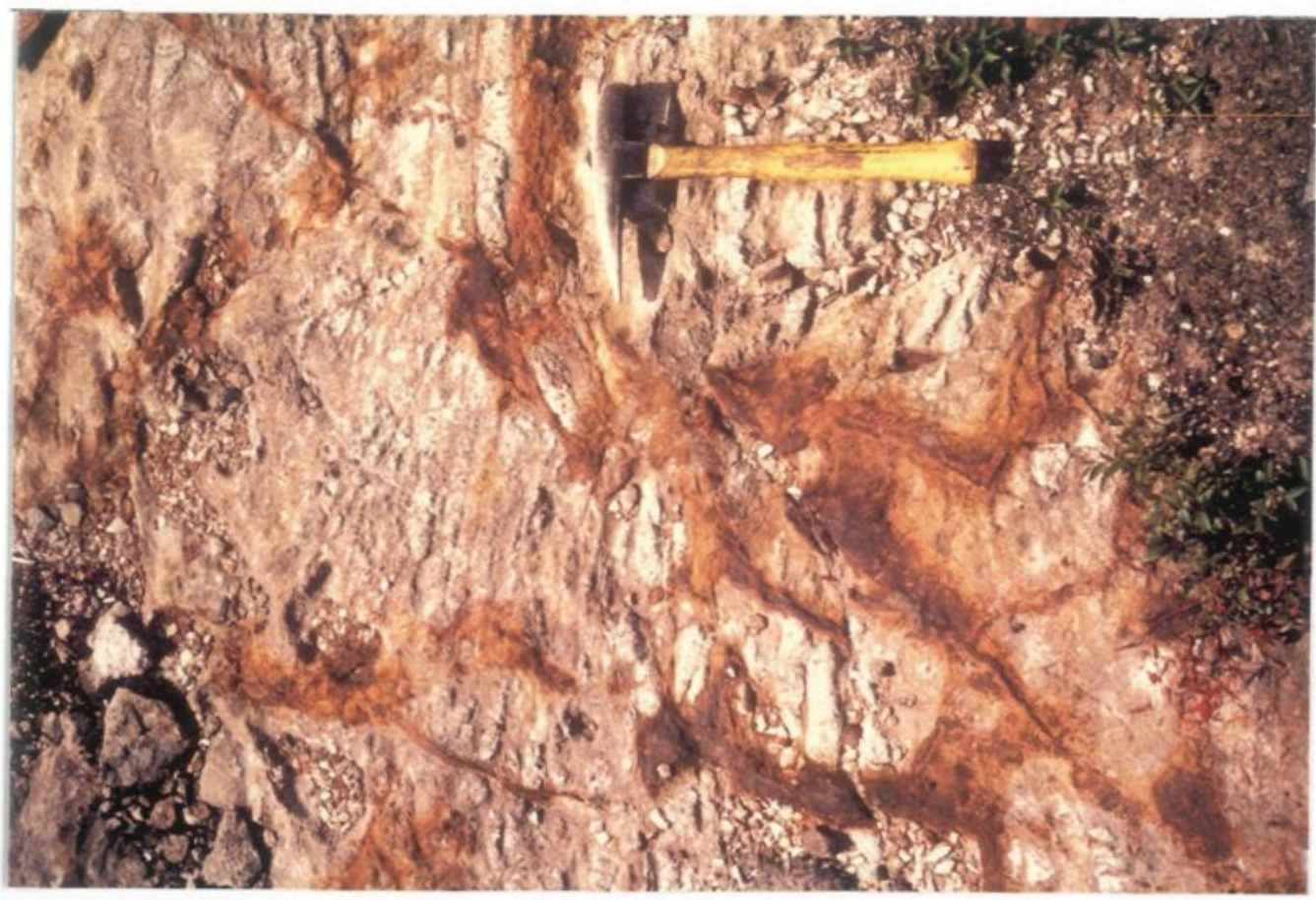

(A)

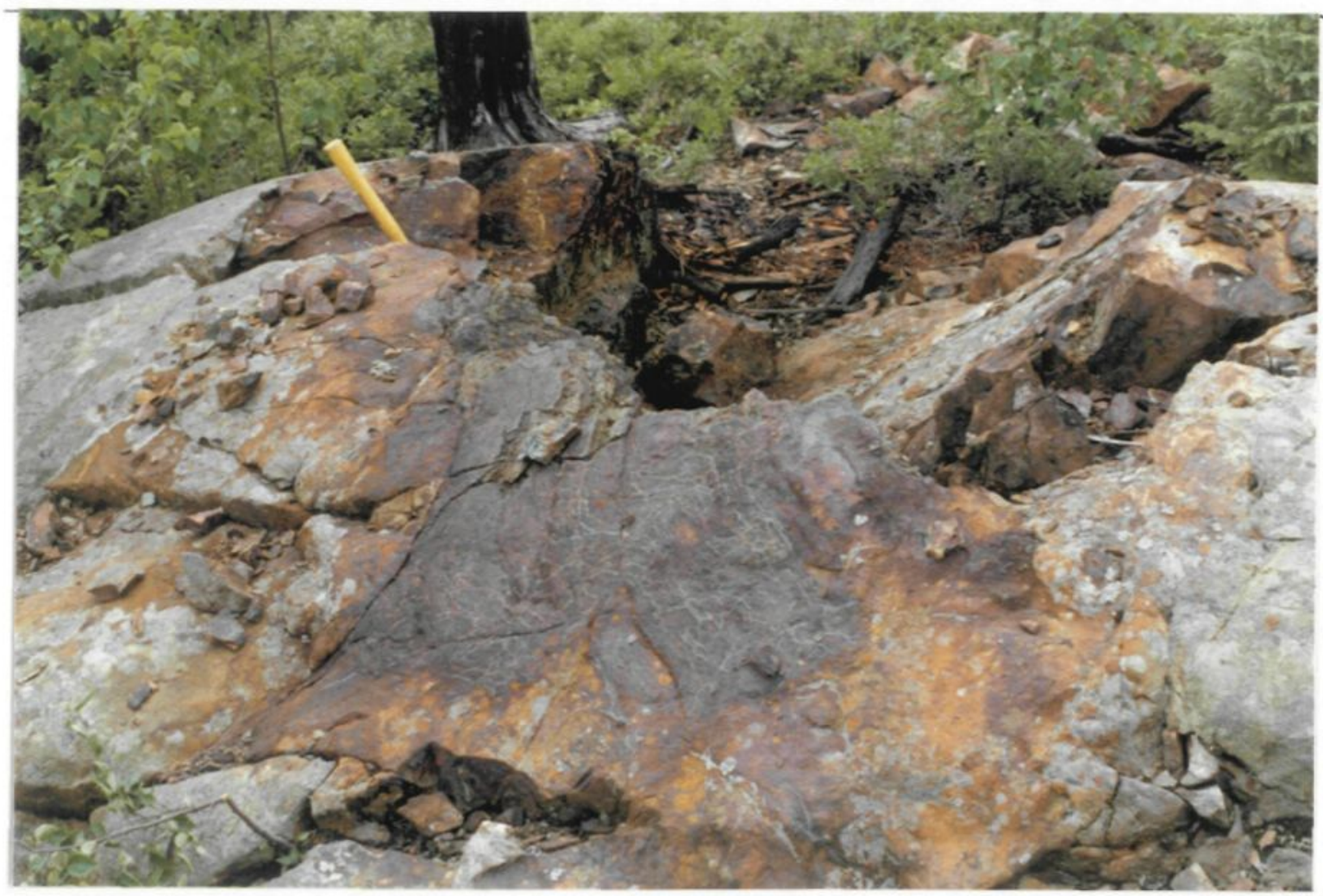


PLANCHE XIX

(A) Début de carbonatisation, de silicification et de chloritisation autour de nucleus constitués de petits fragments et de cristaux. Formation de Blondeau, indice du lac Devilliers.

(B) Intensification du processus d'altération observé sur la photographie précédente. Altérations préférentielles démontrées par la carbonatisation et la silicification, dessinant de pseudo-fragments blanchâtres sur

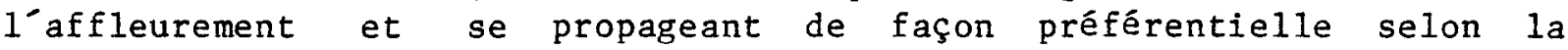
stratification. La chloritisation affecte davantage la matrice (gris verdâtre) et attaque également les zones préalablement carbonatisées et silicifiées, accentuant ainsi $1^{\text {'}}$ aspect de pseudo-fragments observés sur cet affleurement. 


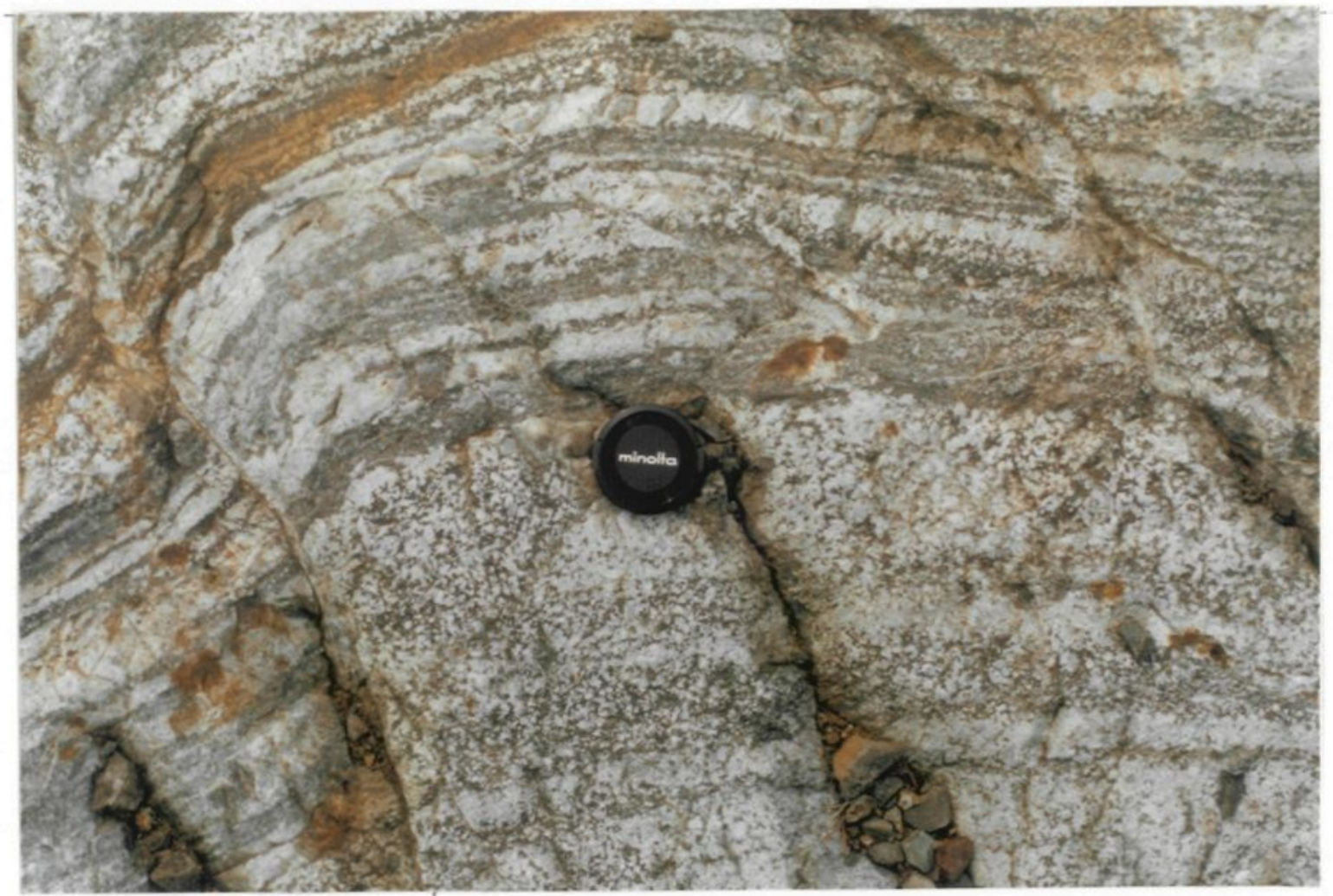

(A)

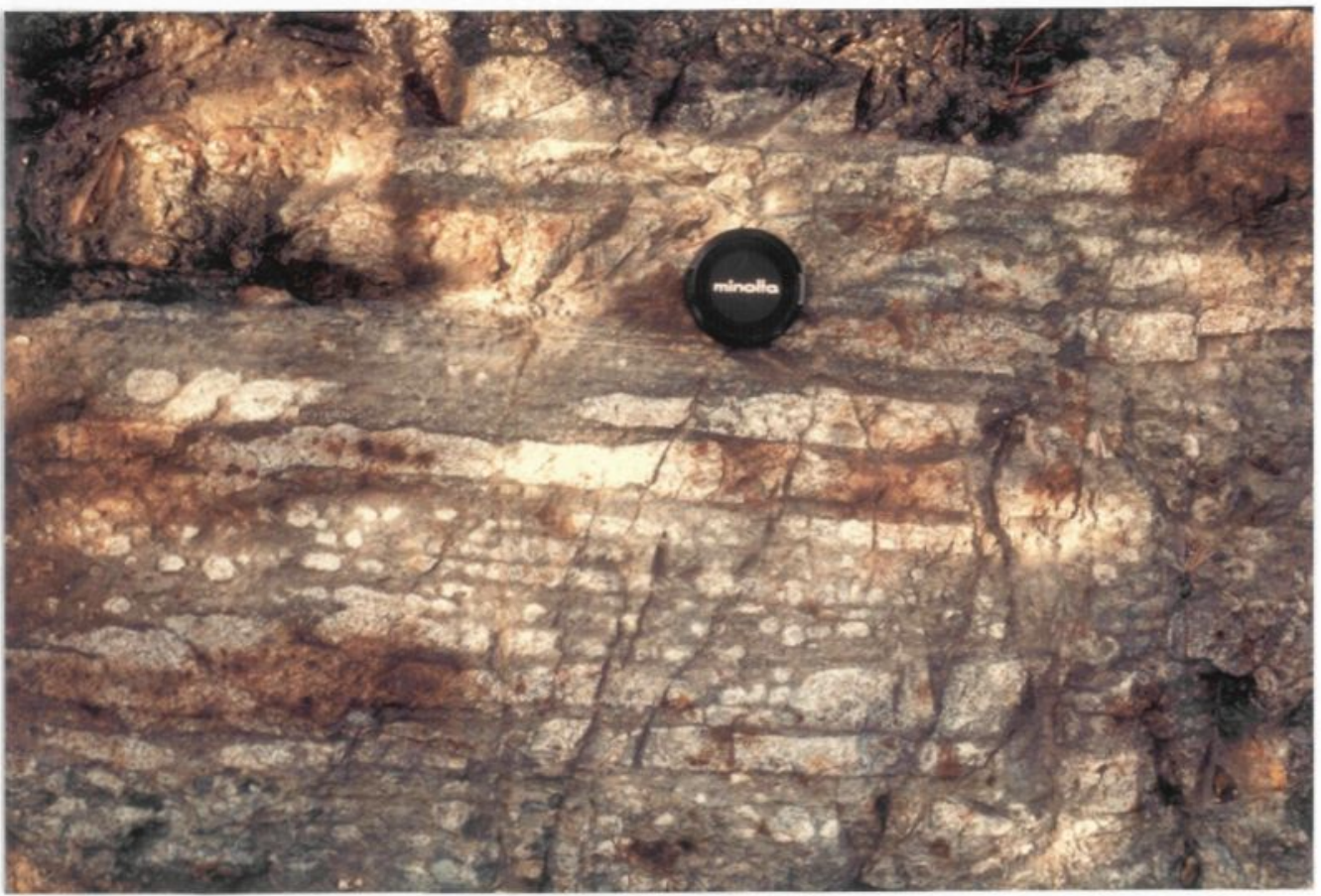




\title{
PLANCHE XX
}

\begin{abstract}
(A) Sédiment volcaniclastique grèseux montrant des pseudo-sphérulites produites par 1'altération hydrothermale. Le coeur du sphérulite est intensément carbonatisé et silicifiée tandis que la matrice est plutôt chloritisée et silicifiée. Formation de Blondeau, indice du lac Devilliers. Lumière naturelle.
\end{abstract}

(B) Même microphotographie que précédemment. La bordure du sphérulite est enrobée de quartz microcristallin. La matrice est intensément cloritisée. Ces caractéristiques témoignent du caractère précoce de la carbonatisation, de la continuité da la silicification et de 1 apparition ultérieure de la chlorite. Lumière polarisée. 


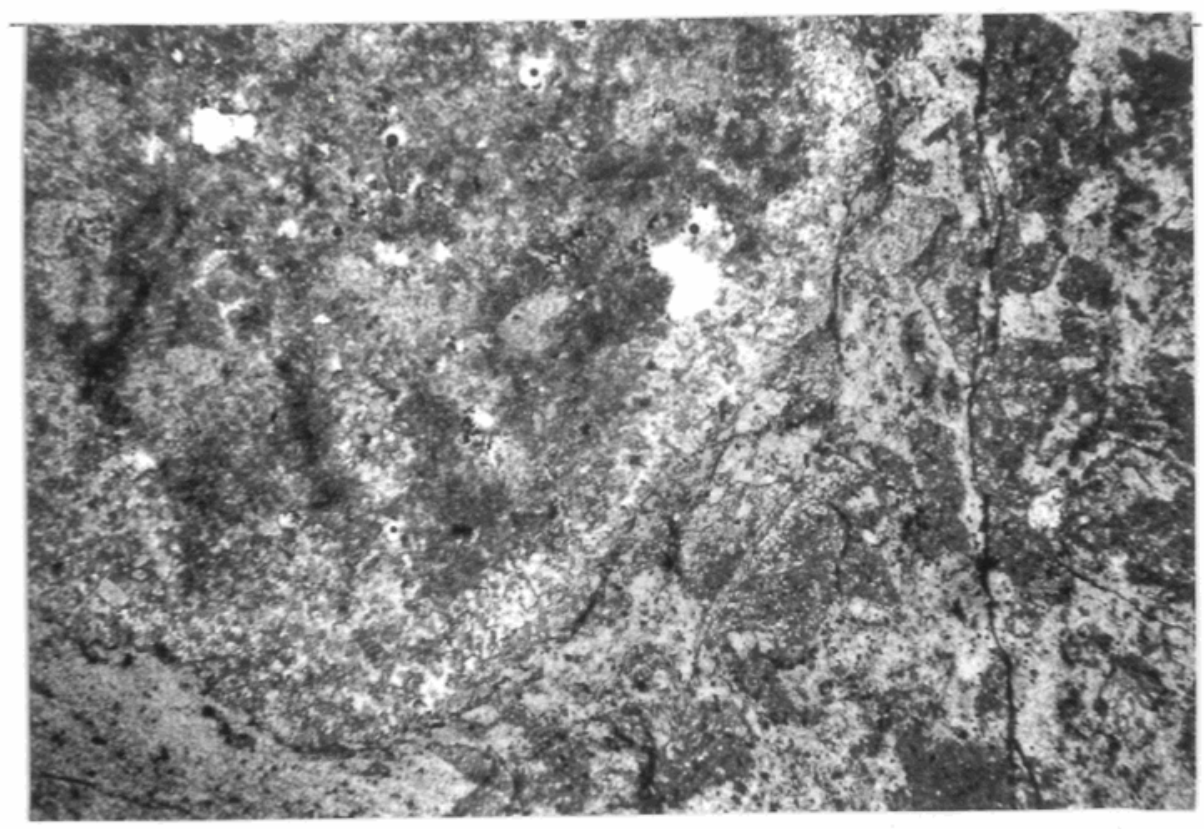

(A)
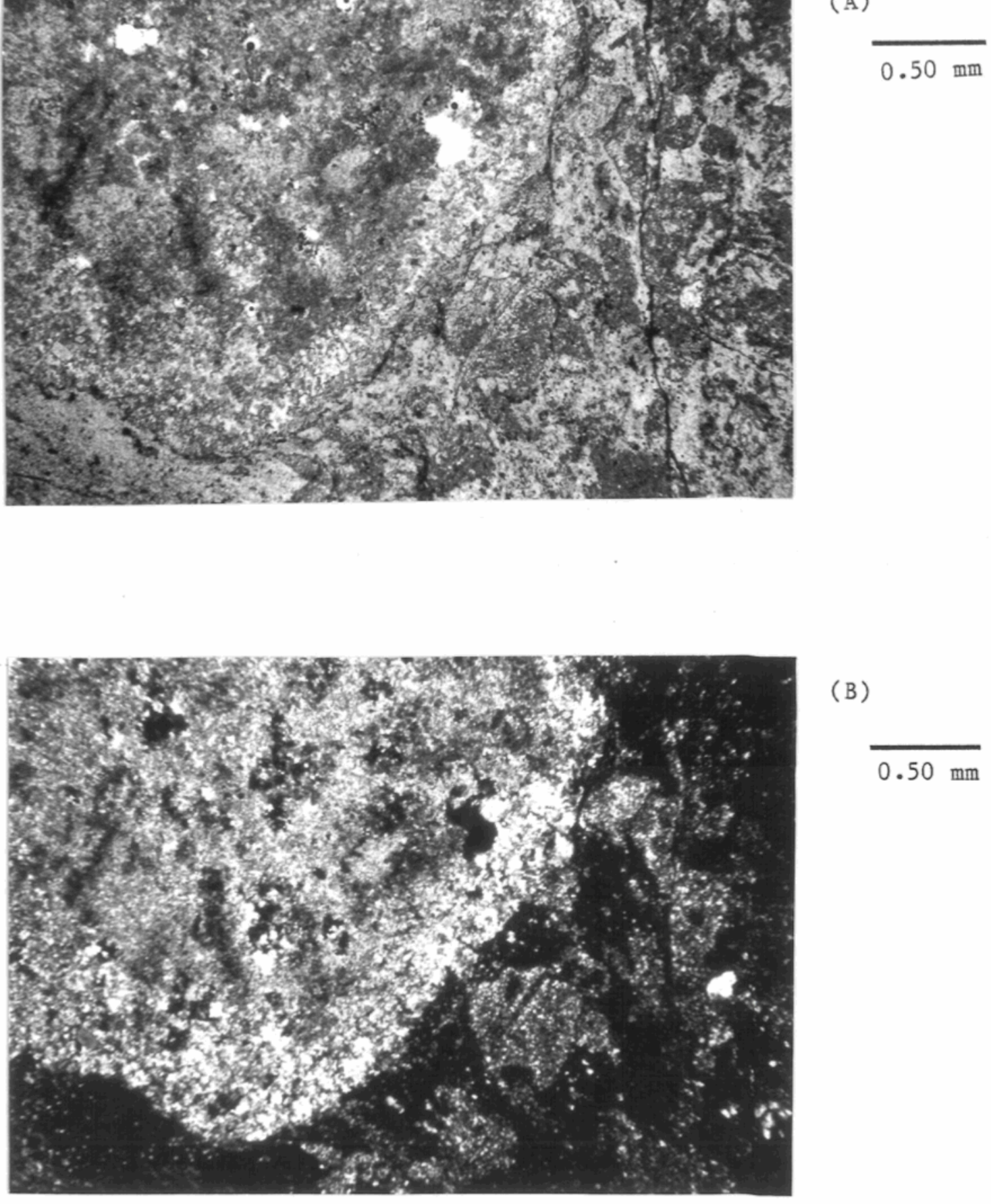

(B)

$0.50 \mathrm{~mm}$ 
(A) Minéralisations rubannées en chalcopyrite (jaune foncé) et en pyrite idiomorphe (jaune pâle). Les paillettes de tourmaline (foncée)

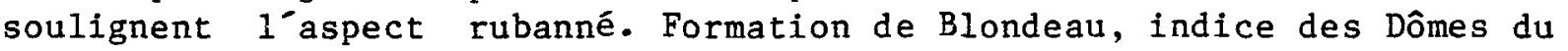
radar, mont Bourbeau. Lumière réfléchie.

(B) Fenêtre au travers du conglomérat protérozoĩque. Celle-ci permet de voir des filonets minéralisés recouverts en discordance par ces sédiments. Indice des Dômes du radar, mont Bourbeau.

(C) Veine de molybdénite (indiquée par la flèche) tapissée de carbonate et encaissée dans un cisaillement d'orientation $\mathrm{N}-\mathrm{S}$. Les épontes sont intensêment silicifiées. Indice des Dômes du radar, mont Bourbeau. 


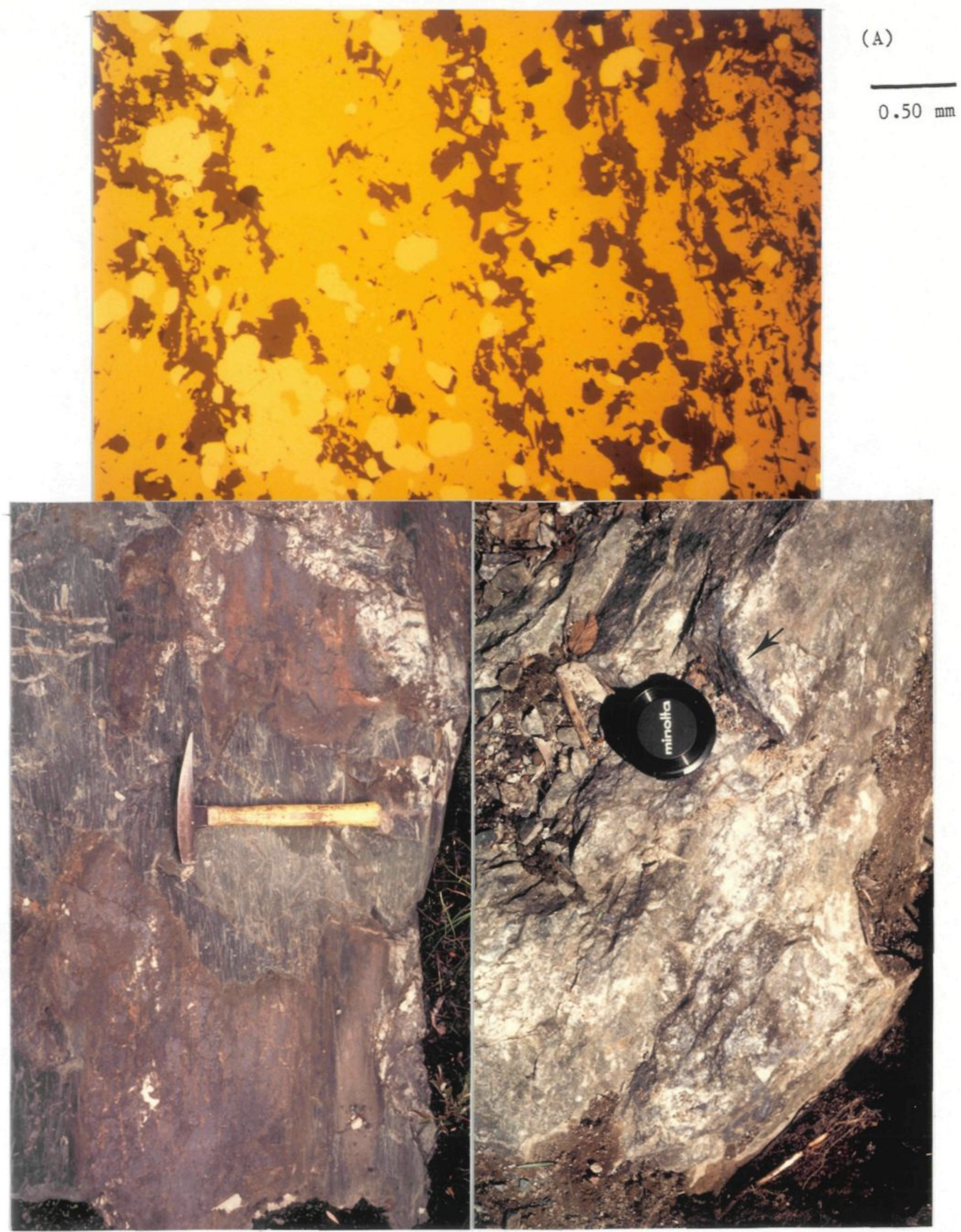

(B)

(C) 
(A) Vue en détail d'un pseudo-fragment de couleur blanchâtre. Une veinule minéralisée en chalcopyrite, sphalérite et pyrite est bordée par une forte altération en chlorite. La séricitisation est observable à partir d'une vingtaine de centimètres de cette veinule. Une seconde fracture, cette fois stérile, borde du côté droit ce pseudo-fragment. Formation de Blondeau, indice d'argent.

(B) Ces veinules minéralisées se composent de sphalérite (gris pâle), contenant fréquemment de fines inclusions de chalcopyrite (jaune foncé), de pyrite (jaune blanchâtre) et de gangue (gris noirâtre). De la millérite (indiquée par la flèche) a possiblement été identifiée. Indice $\mathrm{d}^{\prime}$ argent, lumière réfléchie. 


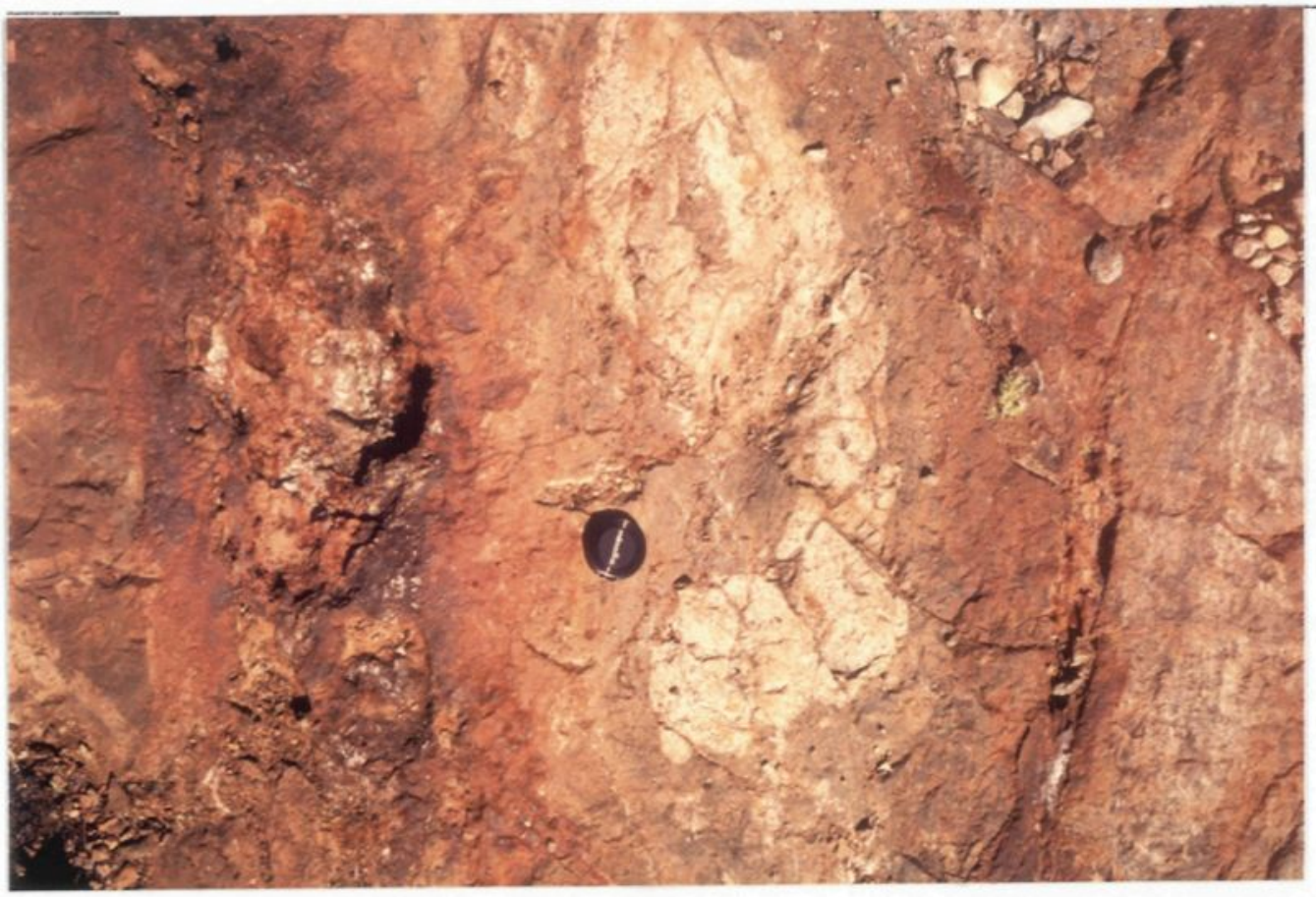

(A)

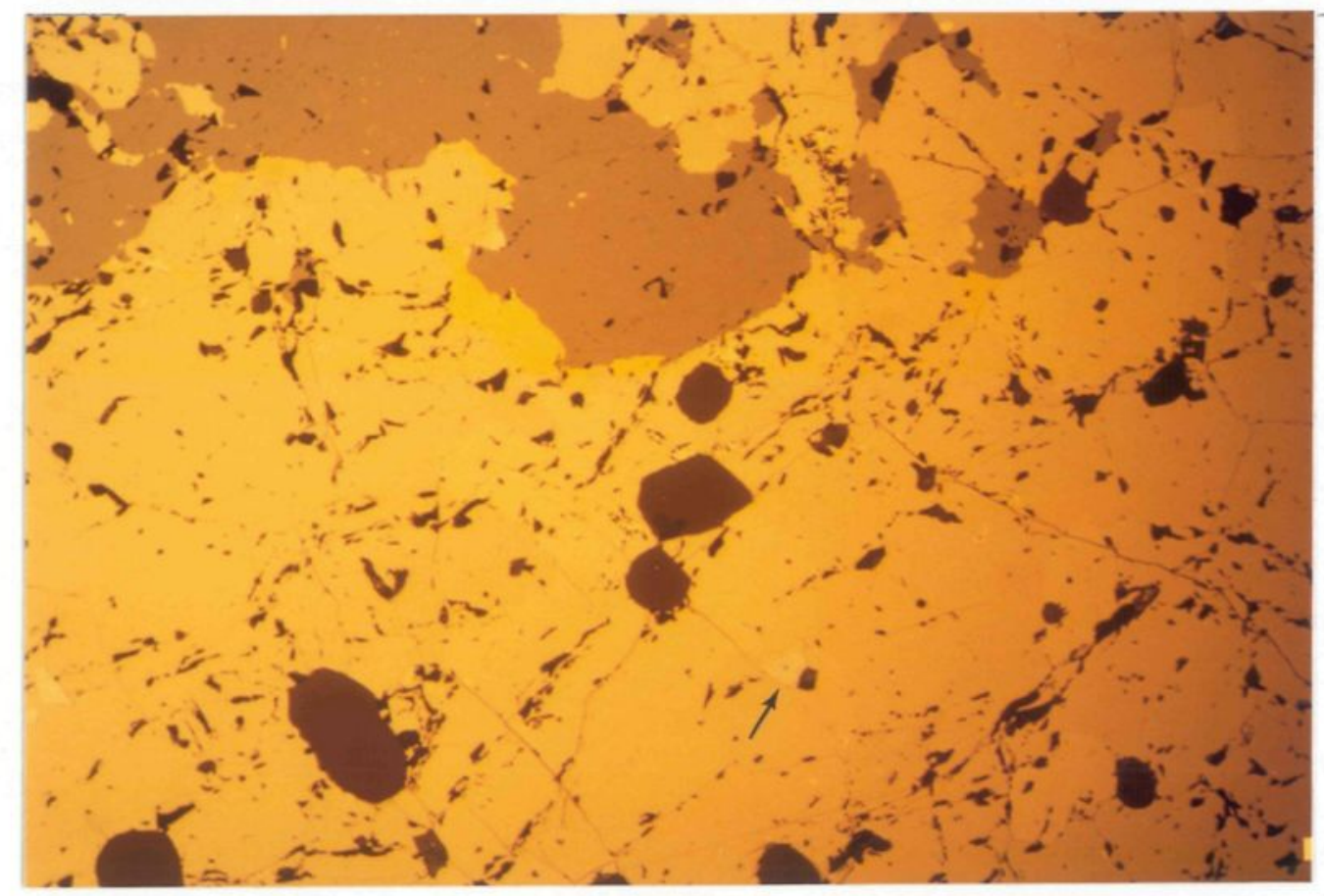

(B)

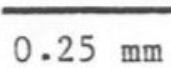


PLANCHE XXIII

(A) Patron de pseudo-bréchification dans les épontes des fractures, causé par la nature distincte et 1 'intensité des altérations en présence: la chloritisation et la séricitisation. Formation de Blondeau, indice d'argent, mont Bourbeau.

(B) Détails sur 1'intensité et la position respective des altérations. Le front de chloritisation (gris-bleuté) se restreint aux bordures immédiates des fractures, minéralisées ou pas, tandis que la séricitisation affecte les roches plus éloignées (blanchâtres). A noter $\mathrm{qu}^{-i} \mathrm{n}^{-} \mathrm{y}$ a pas de déplacement apparent des volcaniclastites de part et d'autre des fractures et des différents fronts d'altérations. La silicification est faible mais affecte une bonne partie de 1 -affleurement. 


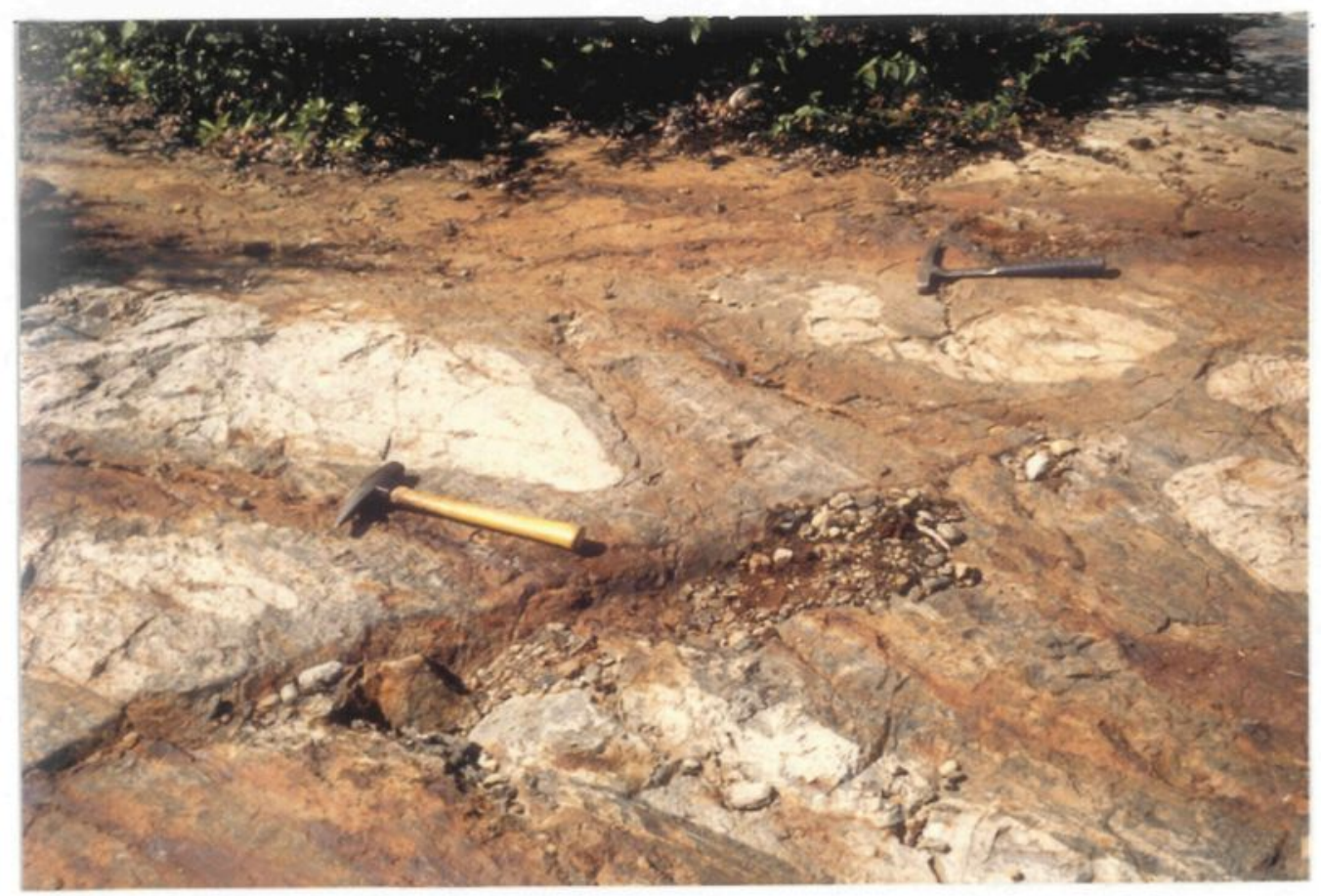

(A)

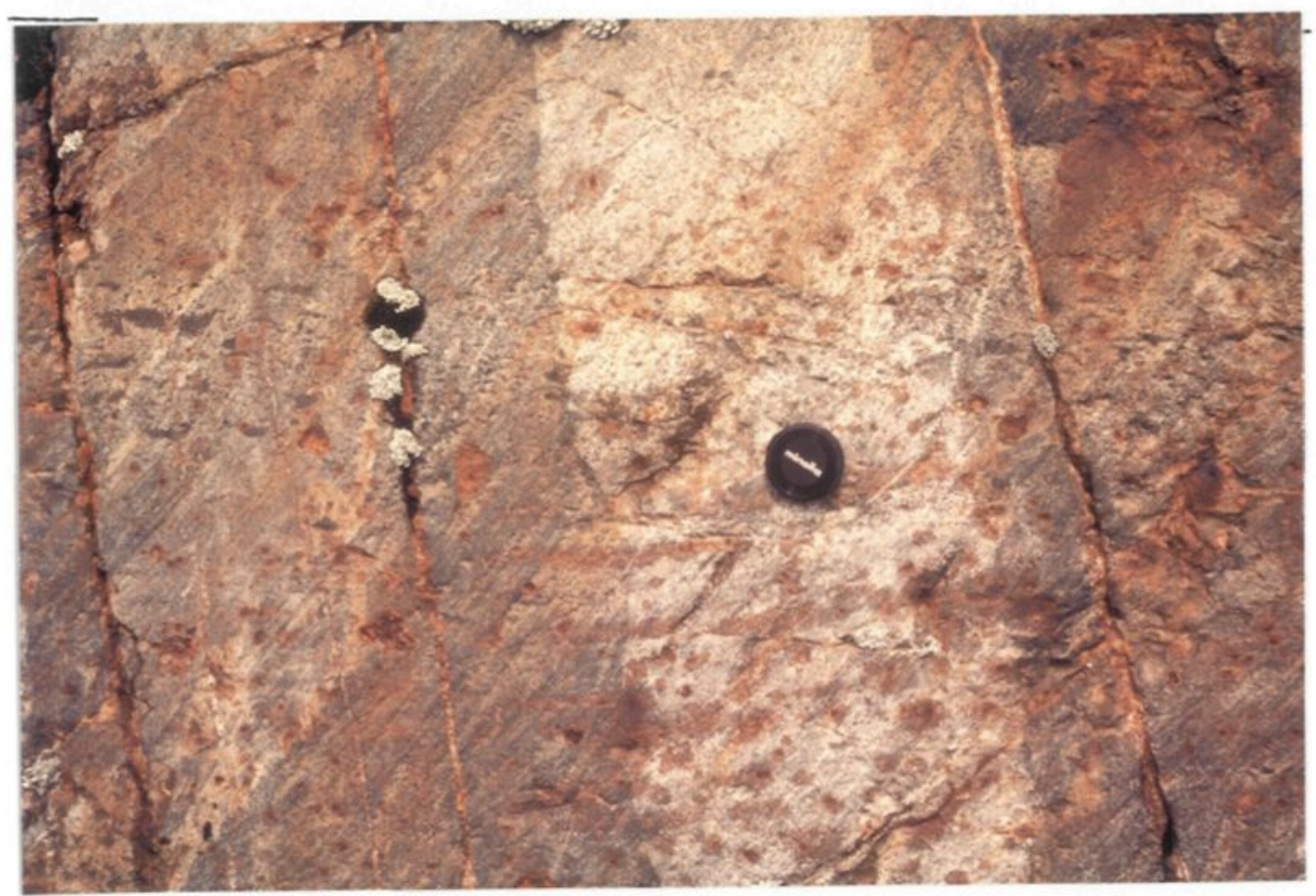

(B) 
PLANCHE XXIV

(A) Coulée pyroclastique de même composition que la roche de la planche XXIV (B) mais ici échantillonnée à l'intérieur du front de séricitisation. Les plagioclases ne laissent que deviner leur silhouette, tant la séricitisation est grande. La matrice est un peu plus chloritisée que dans $1^{\prime}$ exemple précédent. Nicols croisés.

(B) Coulée pyroclastique composée de cristaux libres de plagioclase faiblement séricitisés, d'amphibole et de quartz. La matrice est silicifiée et faiblement chloritisée. Cet échantillon se situe à environ 3 mètres des fractures minéralisées, à 1 'extérieur du front de séricitisation. Formation de Blondeau, indice d’argent. Nicols croisés. 

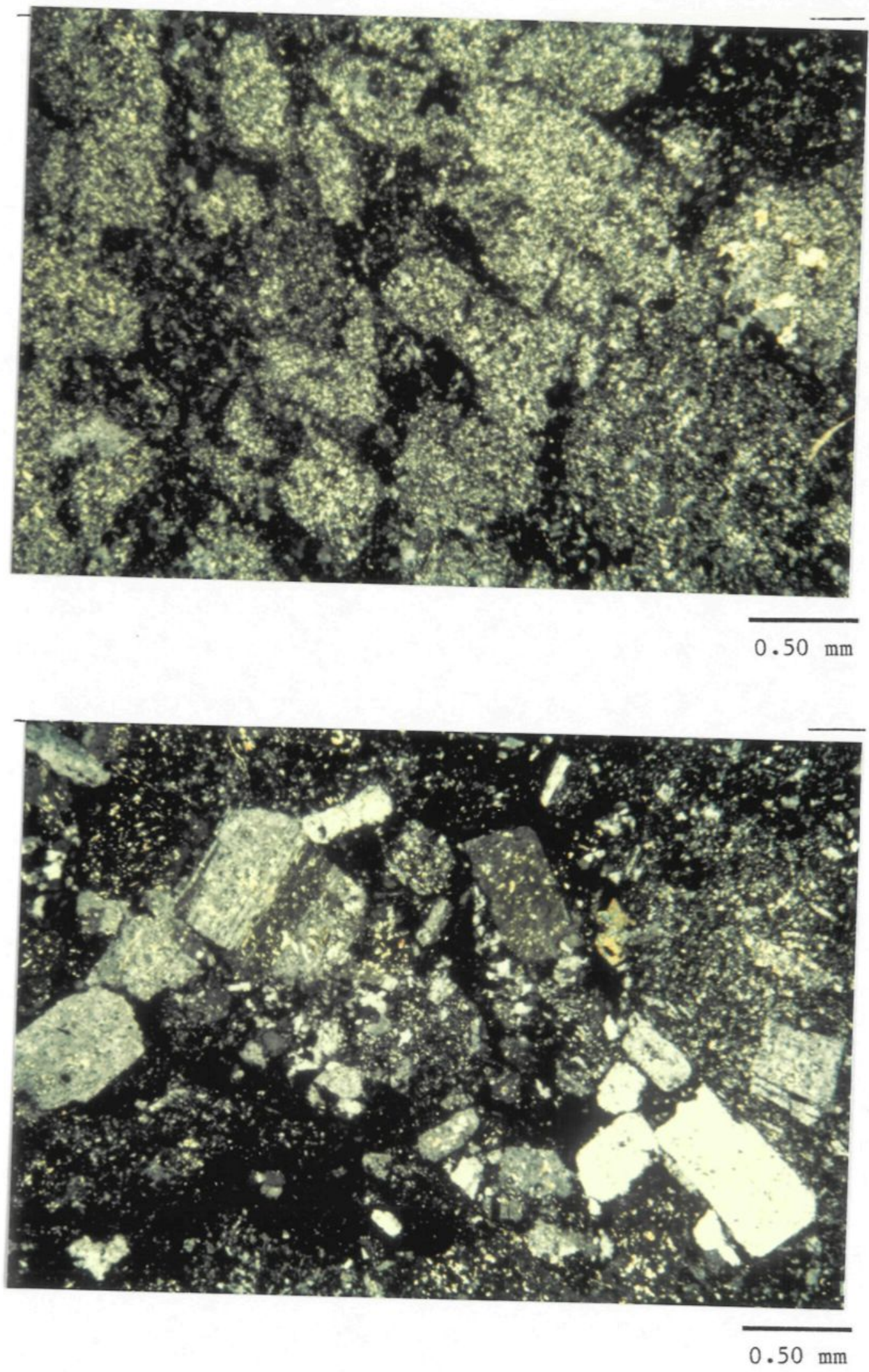
PLANCHE XXV

Faille d'orientation NNW, séparant les couches bien litées de la Formation de Blondeau d'avec la bordure de trempe basale du filon-couche de Bourbeau. Des veines minéralisées sont sub-parallèles à cette structure et se retrouvent préférentiellement du côté des volcaniclastites. 


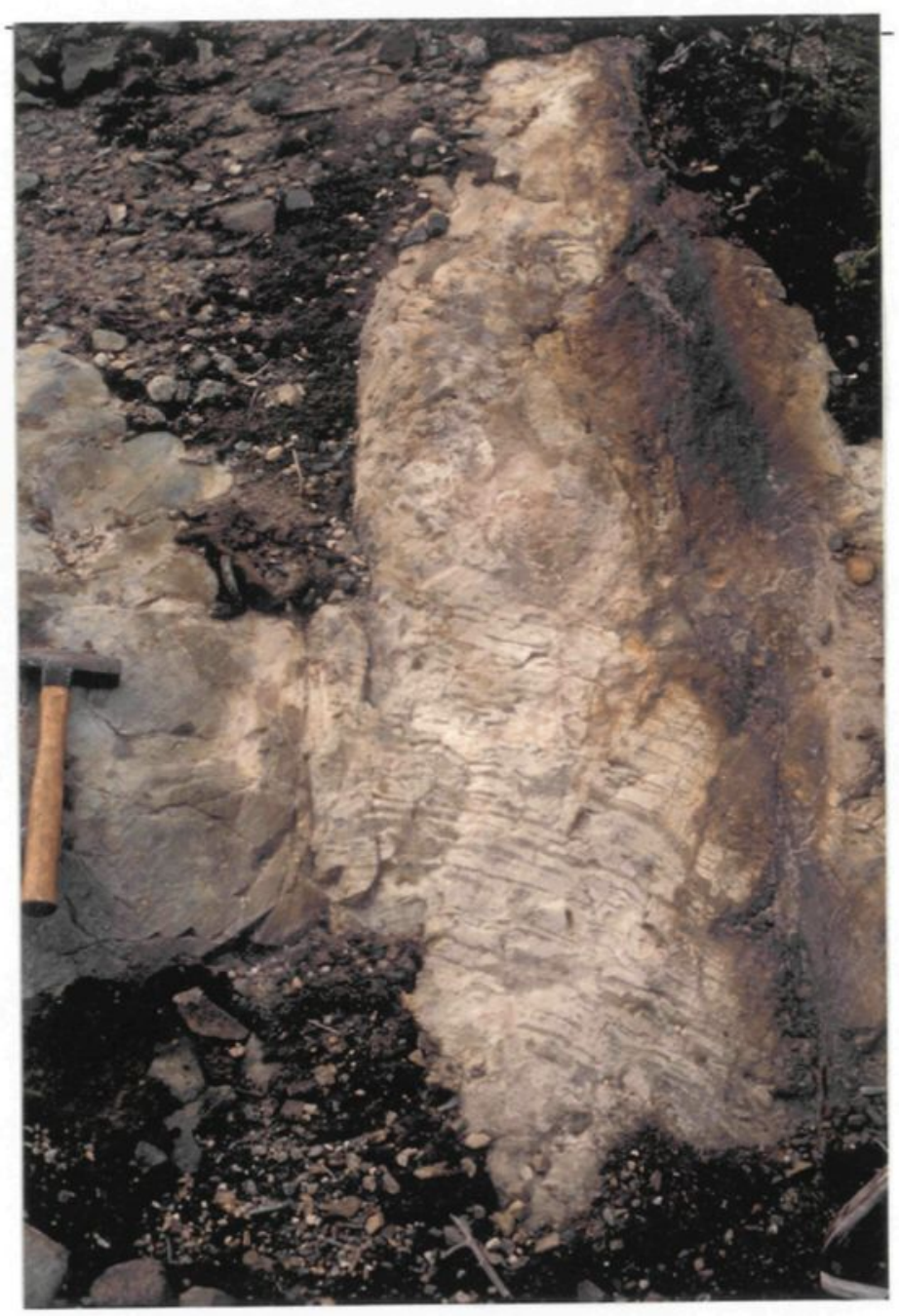




\section{PLANCHE XXVI}

(A) Minéralisations en pyrrhotine, plus ou moins massive, contenant des inclusions de gangue. Dans la Formation de Blondeau, près du sommet du filon-couche de Ventures, mont Bourbeau. Lumière réfléchie.

(B) Même microphotographie que précédemment, nicols croisés. La gangue se compose principalement de quartz microcristallin, de carbonate et de quelques minéraux mafiques totalement chloritisés. 


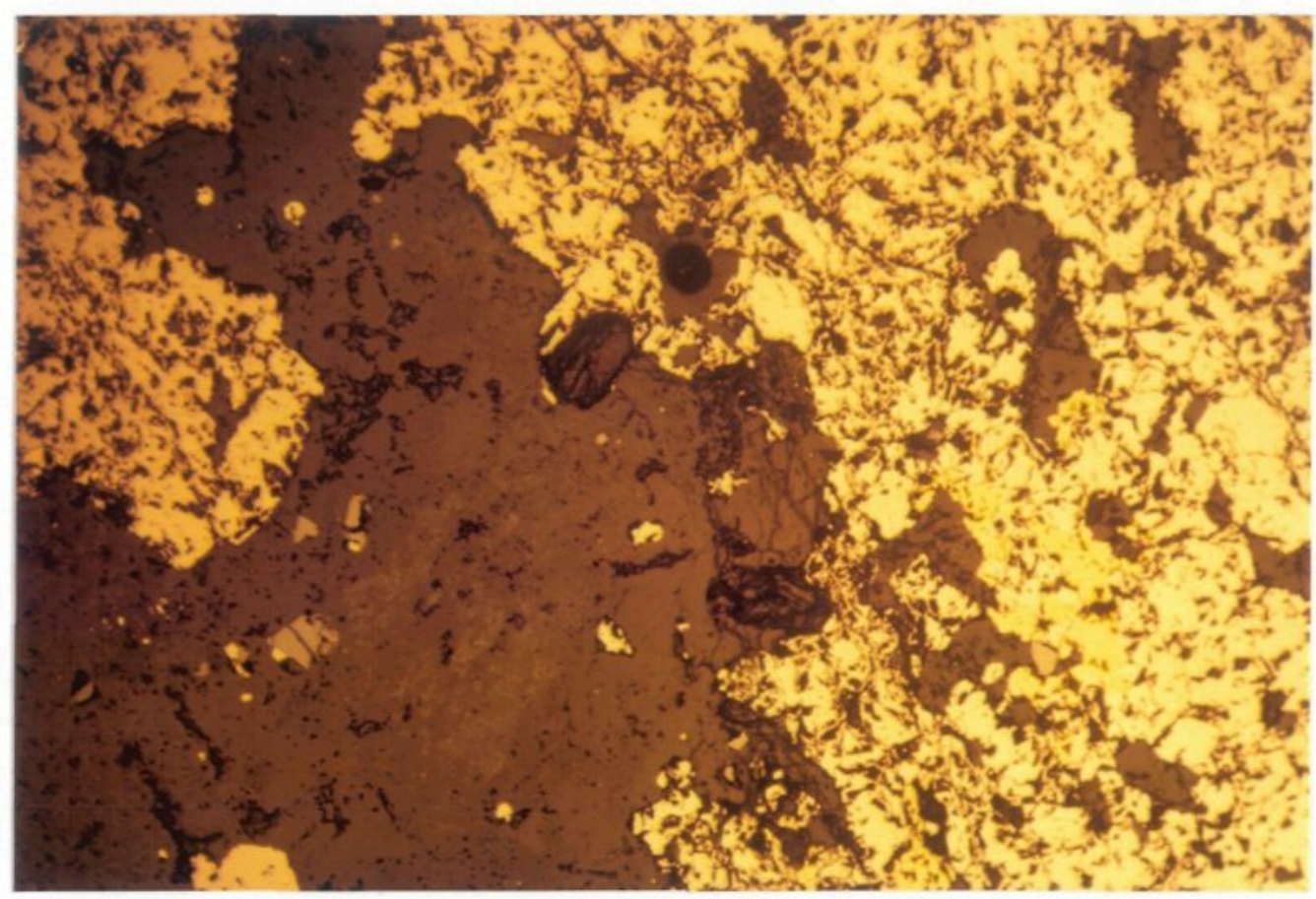

(A)
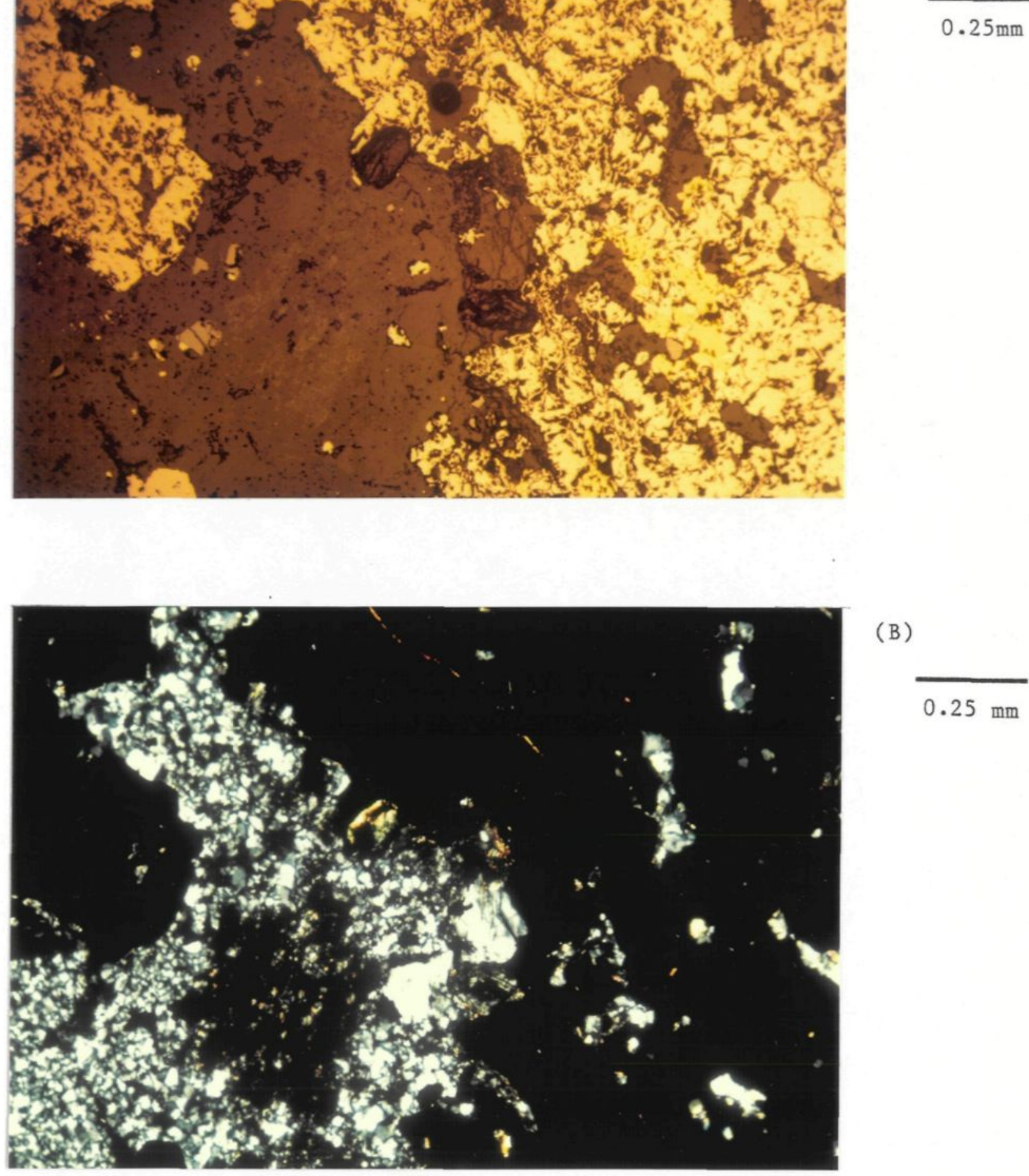

(B)

$0.25 \mathrm{~mm}$ 
PLANCHE XXVII

(A) Minéralisations composées de pentlandite (jaune clair), de chalcopyrite (jaune foncé), de pyrrhotine (gris pâle) et de sphalérite. Des minéraux opaques (phyllosilicates) soulignent un vague rubannement dans la partie droite de la microphotographie. Filon-couche de Ventures, indice "Be1-3", mont Bourbeau. Lumière réfléchie.

(B) Plissotements démontrés par des bandes riches en phyllosilicates bordées par la sphalérite (gris), la pyrrhotine (gris rosé) et la chalcopyrite (jaune foncé). Indice "Bel-3", mont Bourbeau. Lumière réfléchie.

(C) Texture "decussate" présentêe par la pyrrhotine, les bordures en points triples sont fréquentes. Indice "Be1-3", mont Bourbeau. Lumière réfléchie. 


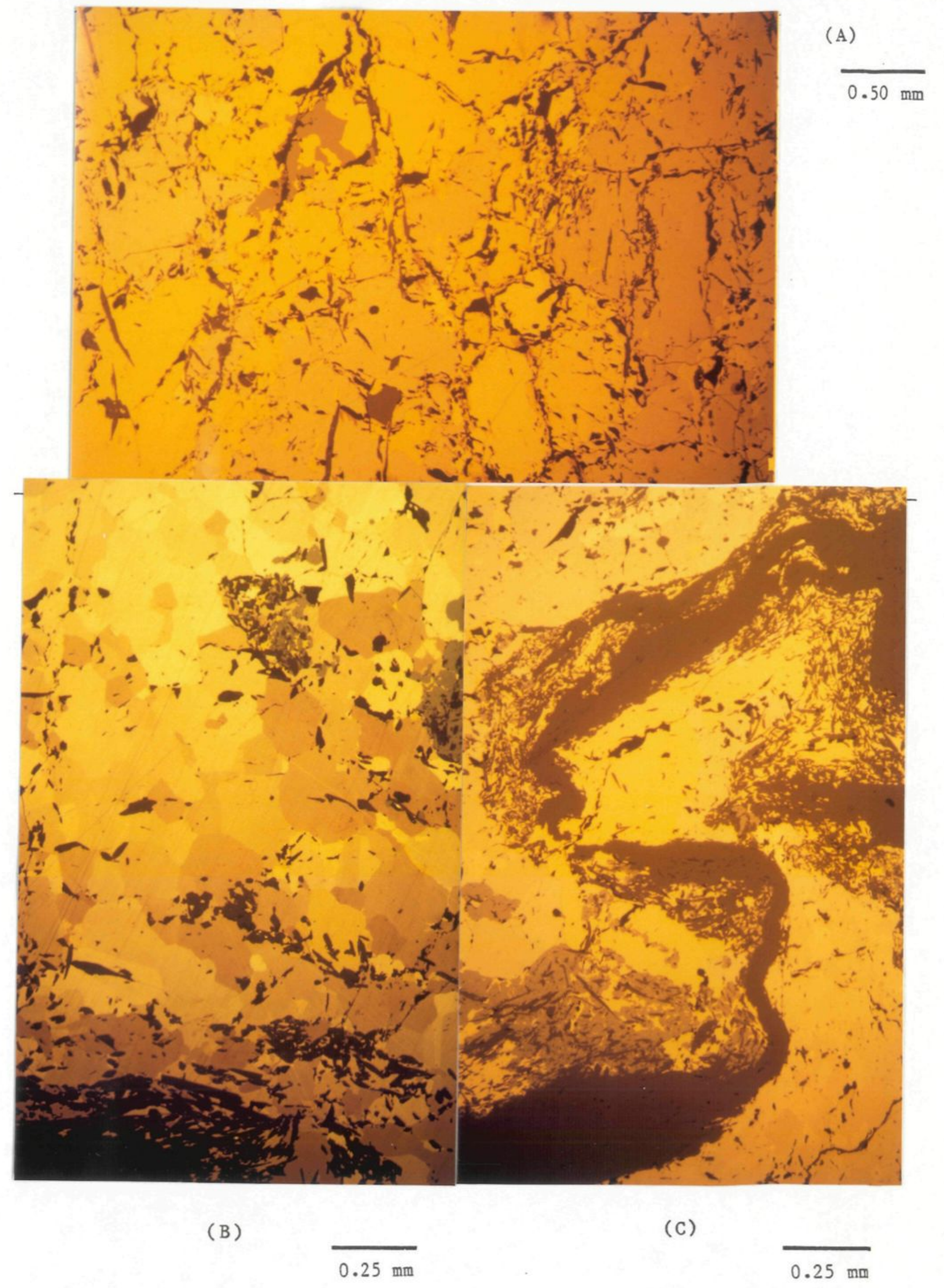


PLANCHE XXVIII

(A) Microphotographie montrant des cristaux aciculaires de molybdénite (jaune pâle) et de pyrite (blanc) enserrés dans une gangue constituée principalement de quartz et de carbonate (jaune grisâtre). Stock tonalitique du lac Line, au sud de la Baie du Cran Penché. Lumière réfléchie.

(B) Brèche dans la leucotonalite, cimentée par un carbonate de fer brunâtre. Présence localement de traces de pyrite. Les fragments, eux-mêmes tonalitiques, sont sub-anguleux et ne semblent pas s'être beaucoup déplacés. Stock tonalitique du lac Line, au nord-ouest du lac Ham. 


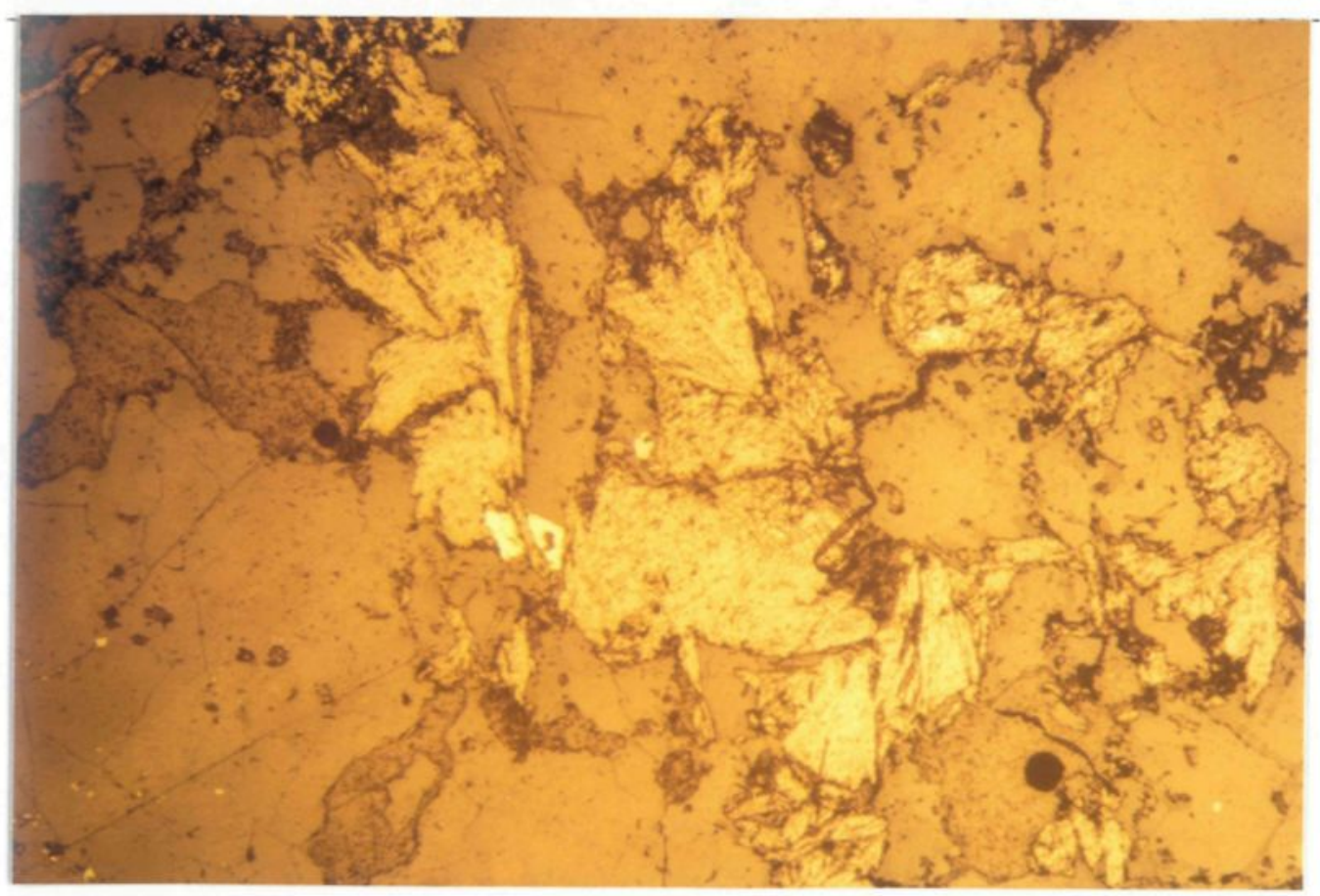

(A)

$$
\overline{0.50 \mathrm{~mm}}
$$

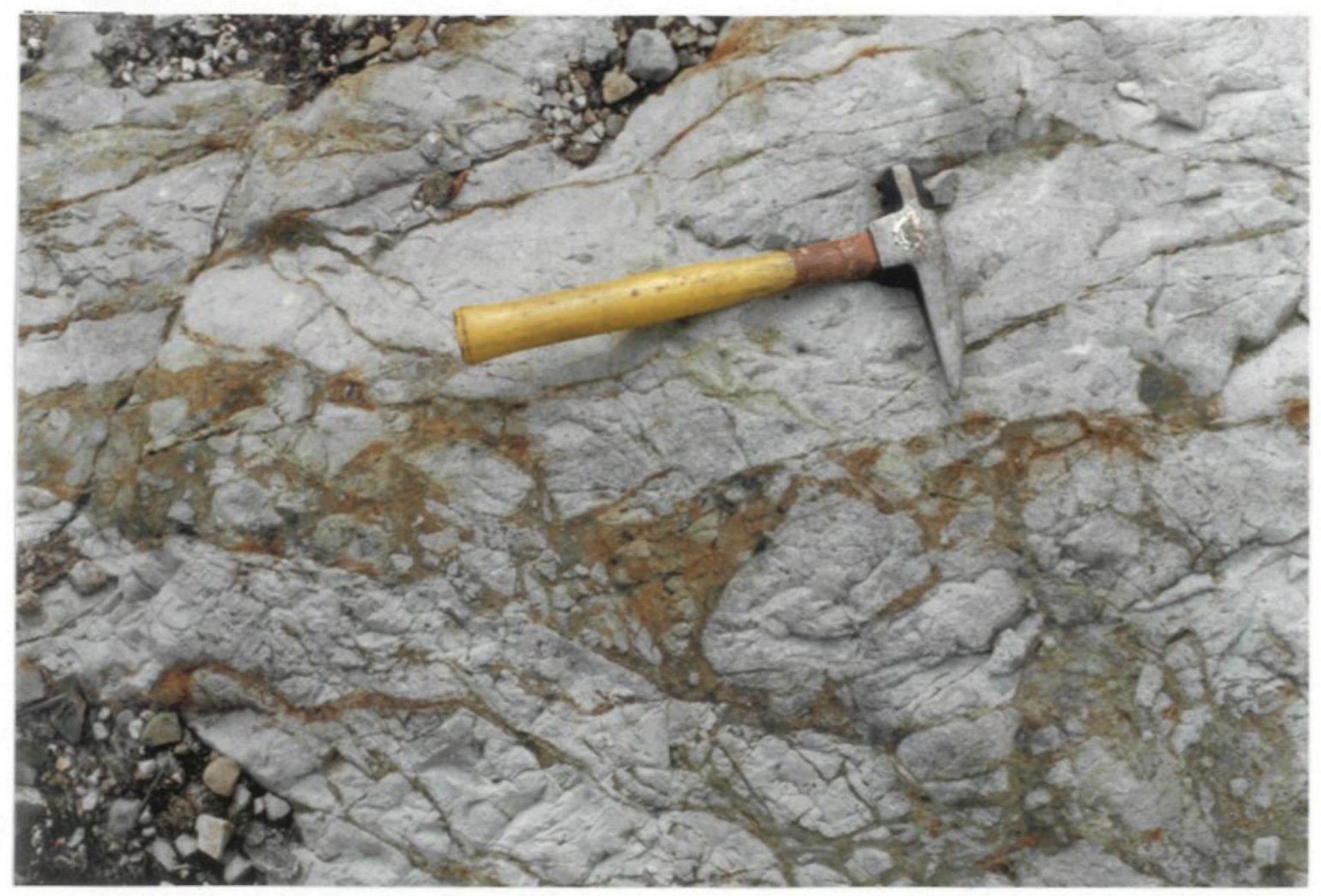


ANNEXE 2

ANALYSES DES ELEMENTS MAJEURS, MINEURS

ET MINERAUX NORMATIFS (CIPN)

DESCRIPTION DES ECHANTILLONS

NUMERO IDENTIFICATION DESCRIPTION

Formation de Gilman

1

92157

2

3

5

6

7

8

9

10

11

12

13

14

15
94674

94685

94679

94681

94680

94682

94684

92149

92148

94662

94675

92142

92152

92164
Basalte, au sud du lac Line

Tuf mafique, peu altéré

Tuf mafique, très altéré

Indice du lac Ham

Basalte (?)

Basalte silicifié et minéralisé

Gabbro en bordure immédiate des veines minéralisées

Gabbro altéré

Gabbro non-altéré, éloigné de la zone minéralisée

Basalte cisaillé

Pyroxénite basale du filon-couche du lac Ham

Formation de Blondeau:

Au sud du lac Larone

Tuf à cristaux

Grès mafique

Basalte variolaire (dyke)

Lits cherteux minéralisés en pyrite

Basalte variolaire 


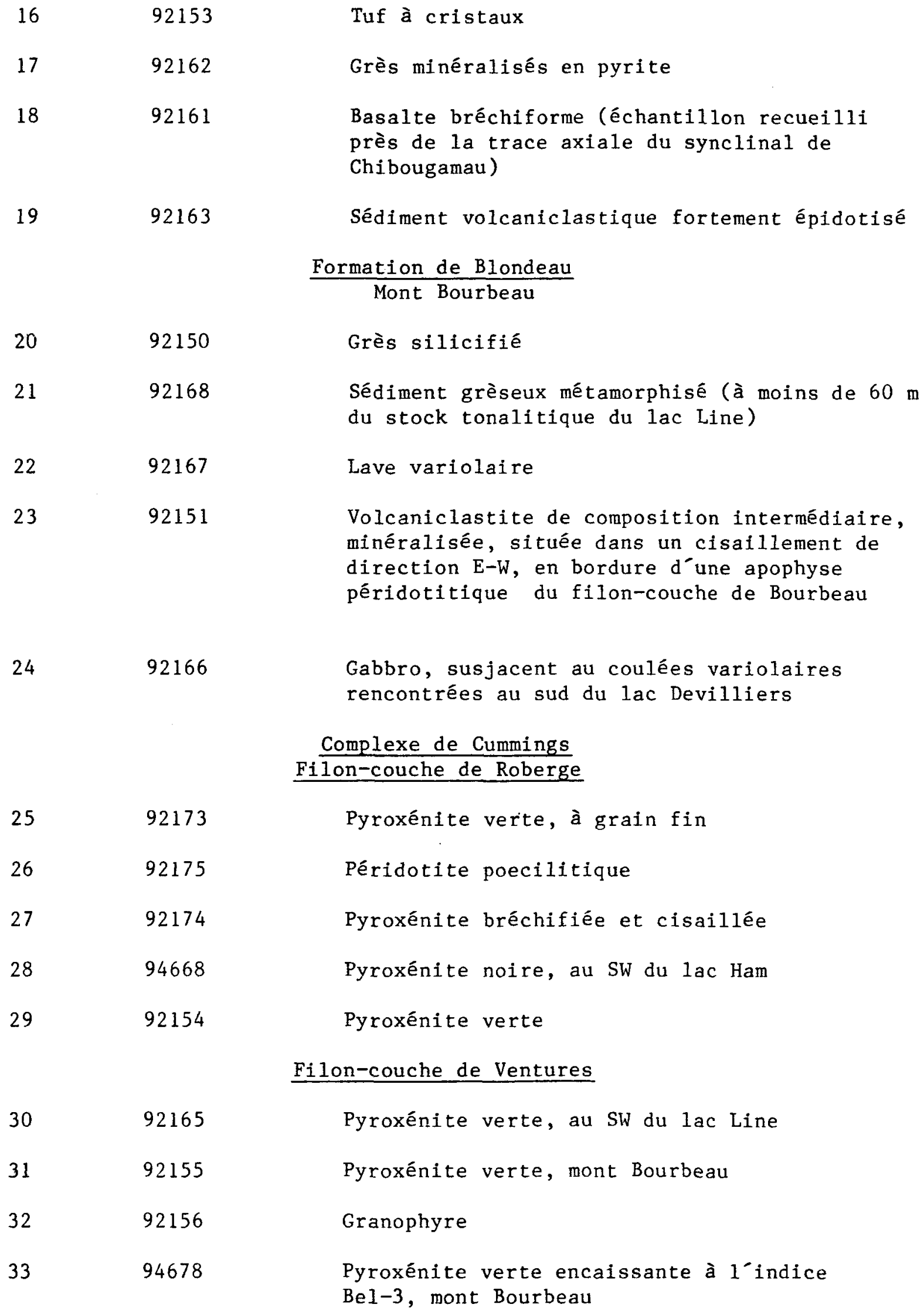

92153

92162

92161

92150

92168

92167

92151

92173

92175

92174

94668

92154

92165

92155

92156

94678

Tuf à cristaux

Grès minéralisés en pyrite

Basalte bréchiforme (échantillon recueilli près de la trace axiale du synclinal de Chibougamau)

Sédiment volcaniclastique fortement épidotisé

\section{Formation de Blondeau}

$$
\text { Mont Bourbeau }
$$

Grès silicifié

Sédiment grèseux métamorphisé (à moins de $60 \mathrm{~m}$ du stock tonalitique du lac Line)

Lave variolaire

Volcaniclastite de composition intermédiaire, minéralisée, située dans un cisaillement de direction $E-W$, en bordure d'une apophyse péridotitique du filon-couche de Bourbeau

Gabbro, susjacent au coulées variolaires rencontrées au sud du lac Devilliers

Complexe de Cummings

Filon-couche de Roberge

Pyroxénite verte, à grain fin

Péridotite poecilitique

Pyroxénite bréchifiée et cisaillée

Pyroxénite noire, au SW du lac Ham

Pyroxénite verte

Filon-couche de Ventures

Pyroxénite verte, au SW du lac Line

Pyroxénite verte, mont Bourbeau

Granophyre

Pyroxénite verte encaissante à $1^{\prime}$ indice Bel-3, mont Bourbeau 
34

94683

94667

35

36

37

38

39

40

41

42

43

44

45

46

47

48

49

94676

92146

92141

92144

92145

92159

92158

94673

92143

94666

94671

94687

94669
Pyroxénite minéralisée en veinules de chalcopyrite, indice Bel-3, mont Bourbeau

Filon-couche de Bourbeau

Bordure de trempe gabbroĩque à la base

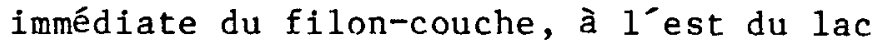
Devilliers

Pyroxénite à "bronzite", à I'est du lac Devilliers

92170 Stock tonalitique du lac Line

Péridotite, mont Bourbeau

Dyke gabbroĩque

Leucogabbro pyroxénitique

Leucogabbro

Ferrodiorite, près du sommet du filon-couche

Ferrogabbro

Leucogabbro

Veine d’actinote, encaisée dans le ferrogabbro

\section{Dykes}

\section{Dyke à phenocristaux de pyroxènes, Formation de Haüy (?)}

Bordure dioritique

Tonalite massive

Tonalite altérée, encaissante aux veines minéralisées, au sud de la Baie du Cran Penché

Syénite porphyrique, provenant de la galerie

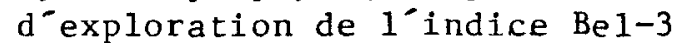




$\begin{array}{llll}\text { SIO2 } & 48.50 & 41.70 & 34.50 \\ \text { AL2O3 } & 14.30 & 10.10 & 12.50 \\ \text { FE203 } & 02.19 & 02.23 & 02.31 \\ \text { FEO } & 09.28 & 05.09 & 04.06 \\ \text { CAO } & 07.54 & 09.60 & 12.70 \\ \text { MEO } & 06.92 & 09.44 & 06.04 \\ \text { NA20 } & 01.66 & 00.04 & 00.13 \\ \text { X2O } & 00.02 & 02.20 & 04.22 \\ \text { TIO2 } & 00.69 & 00.73 & 00.81 \\ \text { CR203 } & 00.00 & 00.10 & 00.13 \\ \text { MNO } & 00.14 & 00.10 & 00.15 \\ \text { P205 } & 00.06 & 00.38 & 00.45 \\ \text { S } & 00.07 & 00.17 & 00.03 \\ \text { CO2 } & 00.00 & 00.00 & 00.00 \\ \text { H2OT } & 06.92 & 16.83 & 20.07 \\ & & & \\ & 98.29 & 98.71 & 98.10\end{array}$

\begin{tabular}{|c|c|c|c|}
\hline Cu (ppm) & 9.7 & 9 & 190 \\
\hline $2 n$ & 140 & 80 & 44 \\
\hline $\mathrm{Pb}$ & $\therefore 5$ & $<.5$ & $<.5$ \\
\hline $\mathrm{Ni}$ & 93 & 410 & 310 \\
\hline Co & & 62 & 25 \\
\hline $\mathrm{Cr}$ & & * & * \\
\hline v & & 160 & 170 \\
\hline$W$ & & $<2$ & $<2$ \\
\hline $3 n$ & & 2 & 1 \\
\hline As & & 40 & 21 \\
\hline Mo & & 43 & 43 \\
\hline $\begin{array}{l}A u \quad C p \\
A g\end{array}$ & $\begin{array}{r}<15 \\
<250\end{array}$ & $\begin{array}{r}<15 \\
\langle 250\end{array}$ & $\begin{array}{r}<15 \\
<250\end{array}$ \\
\hline
\end{tabular}


LAC HAM

94679946819468094682946849214992148

\begin{tabular}{|c|c|c|c|c|c|c|c|}
\hline $10:$ & 41.30 & 59 & 50 & 0 & 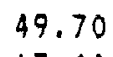 & & 51 \\
\hline 3 & 04 & 30 & 12.70 & 12.10 & 13.40 & 02.81 & 09.0 \\
\hline 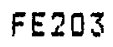 & 01.95 & 02.00 & 03.29 & 25 & 03.38 & 01.88 & \\
\hline EO & 15 & 07.56 & 06.49 & 09.22 & & 54 & 06.6 \\
\hline 80 & 09.03 & 01.81 & 08.18 & 08.93 & 07.29 & 08.20 & 12.60 \\
\hline 0 & 18.50 & 01.20 & 02.82 & 03.97 & 04.43 & 13 & 10. \\
\hline NA2D & 4 & 06. & 04.79 & 7 & 04. & 9 & I. \\
\hline 20 & 00.12 & 00.13 & 00.11 & 01 & 00.48 & 00 & 00. \\
\hline 102 & 00.45 & 00.50 & 01.79 & 01.75 & 01.88 & 00.38 & 00. \\
\hline$R 203$ & 00.22 & 00.00 & 00.00 & 00,00 & 00.00 & 00.00 & 00.3 \\
\hline vo & 00.11 & 00.03 & 00.09 & 00.12 & 00.14 & 00.12 & 50. \\
\hline 105 & 00.03 & 00.10 & 00.18 & 3 & 00.20 & 00 & I. \\
\hline & 00 & 04.07 & 08 & & 00.01 & & \\
\hline $\mathrm{CO} 2$ & 00.00 & 00.00 & 00.00 & 00.00 & 00.00 & 00.00 & 00. \\
\hline$-120 T$ & 04.32 & 00.78 & 07.00 & 02.22 & 02.00 & 00.76 & \\
\hline
\end{tabular}

QUART2 $00.00 \quad 11.16 \quad 05.02 \quad 00.00 \quad 00.00 \quad 00.00 \quad 00.00$

ORTHO. $\quad 00.73 \quad 00.75 \quad 00.66 \quad 08.70 \quad 02.91 \quad 00.00 \quad 01.14$

ALBIT $\quad 01.22 \quad 56.24 \quad 40.84 \quad 34.50 \quad 40.76 \quad 00.00 \quad 24.25$

ANORTH $\quad 11.33 \quad 07.71 \quad 12.93 \quad 10.42 \quad 14.47 \quad 14.33 \quad 11.49$

NEPHEL $\quad 00.00 \quad 00.00 \quad 00.00 \quad 00.00 \quad 00.00 \quad 00.01 \quad 00.00$

LEUC!T $00.00 \quad 00.00 \quad 00.00 \quad 00.00 \quad 00.00 \quad 00.01 \quad 00.00$

WOLLAS $00.00 \quad 00.00 \quad 00.00 \quad 00.00 \quad 00.00 \quad 00.00 \quad 00.00$

DIOPSI $28.24 \quad 00.32 \quad 22.08 \quad 26.91 \quad 17.71 \quad 71.08 \quad 41.25$

HYPERS $\quad 17.65 \quad 07.51 \quad 02.50 .05 .58 \quad 06.62 \quad 00.0009 .55$

OLIVIN $31.38 \quad 00.00 \quad 00.00 \quad 01.11 \quad 06.25 \quad 01.76 \quad 04.79$

ACMITE $00.00 \quad 00.00 \quad 00.00 \quad 00.00 \quad 00.00 \quad 00.00 \quad 00.00$

CORIND $\quad 00.00 \quad 00.00 \quad 00.00 \quad 00.00 \quad 00.00 \quad 00.00 \quad 00.00$

ILMENI $00.88 \quad 00.92 \quad 03.43 \quad 03.33 \quad 03.67 \quad 01.75 \quad 01.69$

MAGNET $02.92 \quad 02.82 \quad 04.81 \quad 04.72 \quad 05.03 \quad 06.62 \quad 03.49$

HEMATI $00.00 \quad 00.00 \quad 00.00 \quad 00.00 \quad 00.00 \quad 00.00 \quad 00.00$

APATIT $00.07 \quad 00.23 \quad 00.43 \quad 00.55 \quad 00.4900 .23 \quad 00.12$

CHROMI $00.33 \quad 00.00 \quad 00.00 \quad 00.00 \quad 00.00 \quad 00.00 \quad 00.46$

CALCIT $00.00 \quad 00.00 \quad 00.00 \quad 00.00 \quad 00.00 \quad 00.00 \quad 00.00$

PYRITE $00.77 \quad 11.59 \quad 00.26 \quad 01.95 \quad 00.04 \quad 02.36 \quad 00.04$

H2O T $04.46 \quad 00.76 \quad 07.05 \quad 02.22 \quad 02.05 \quad 01.85 \quad 01.72$

$99.98100 .01100 .0199 .99 \quad 100 \quad 10099.99$

NOTE: 1) FeO \% = (Fe203\%(Fe tot $)-T 102 \%+1.5) \times .89998$

2) $\mathrm{Fe} 203 \%=\mathrm{TiO2}+1.5$

3) $\mathrm{H} 2 \mathrm{O} T$ = Perte au feu $\left(P, A_{1} F_{\text {. }}\right)-\mathrm{CO} 2 \%$,

si $602 \%>$ P.A.F. alors P.A.F. est estimee dgaler zero 
94679946819468094682946849214992149

\begin{tabular}{|c|c|c|c|c|c|c|c|}
\hline Cu $(p p$ n & 21 & 53 & 12 & 130 & 50 & 130 & 13 \\
\hline Zn & 130 & 27 & 95 & 74 & 100 & 120 & 100 \\
\hline $\mathrm{Po}$ & $<.5$ & 5 & $<.5$ & $<.5$ & $<.5$ & $<.5$ & $<.5$ \\
\hline$N i$ & 660 & 31 & 35 & 34 & 23 & 280 & \\
\hline Co & 38 & 71 & 37 & 84 & 31 & & \\
\hline $\mathrm{Cr}$ & * & 25 & 8 & 5 & 4 & & \\
\hline V & 94 & 53 & 200 & 280 & 300 & & \\
\hline$W$ & $\langle 2$ & $<2$ & 2 & $\vdots 2$ & $\zeta 2$ & & \\
\hline $5 n$ & 5 & 3 & 3 & 2 & 2 & & \\
\hline As & 138 & $.30 \%$ & 1 & 3 & 1 & & \\
\hline 110 & $<3$ & 43 & 9 & 43 & $<3$ & & \\
\hline$A u(p p b)$ & $<15$ & 615 & $<15$ & $<15$ & 415 & 15 & 45 \\
\hline$A g$ & $<250$ & 750 & $<250$ & $<250$ & $<250$ & $\angle 250$ & $<25$ \\
\hline
\end{tabular}


FORMATION DE BLONDEAU

$\$ 102 \quad 63.30 \quad 61.60 \quad 49.70 \quad 52.10 \quad 50.20 \quad 60.60 \quad 49.60 \quad 55.60 \quad 58.40$ $\begin{array}{lllllllllll}\text { AL203 } & 14.80 & 15.40 & 12.70 & 10.80 & 14.10 & 17.40 & 16.80 & 11.90 & 17.50\end{array}$

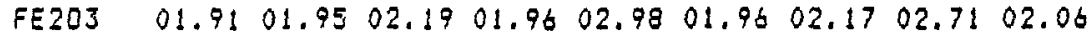
FED

CAO

MGO

NA2O

k.20

$\mathrm{TIO2}$

CR203

MNO

P205

$\mathrm{S}$

$\mathrm{CO} 2$

$\mathrm{H} 2 \mathrm{OT}$

$\begin{array}{lllllllll}04.71 & 05.21 & 07.21 & 16.33 & 08.84 & 03.96 & 04.61 & 05.71 & 05.24\end{array}$ $\begin{array}{llllllllll}0 & 0.51 & 02.97 & 10.70 & 00.47 & 05.90 & 01.95 & 04.08 & 05.96 & 02.86\end{array}$ $\begin{array}{llllllllll}03.53 & 01.00 & 10.10 & 01.55 & 06.84 & 01.58 & 11.10 & 03.72 & 03.15\end{array}$ $\begin{array}{llllllllll}0 & 0.41 & 00.09 & 01.89 & 00.67 & 04.16 & 05.04 & 03.70 & 03.10 & 06.08\end{array}$ $\begin{array}{lllllllllll}03.44 & 04.31 & 02.29 & 02.32 & 00.20 & 02.47 & 01.32 & 00.64 & 00.09\end{array}$ $\begin{array}{llllllllll}0 & 0.41 & 00.45 & 00.69 & 00.46 & 01.48 & 00.46 & 00.67 & 01.21 & 00.56\end{array}$ $00.00 \quad 00.00 \quad 00.00 \quad 00.37 \quad 00.0000 .00 \quad 00.0900 .0000 .00$ $\begin{array}{llllllllll}00.15 & 00.19 & 00.18 & 00.04 & 00.11 & 00.05 & 00.08 & 00.22 & 00.09\end{array}$ $00.08 \quad 00.10 \quad 00.18 \quad 00.01 \quad 00.16 \quad 00.14 \quad 00.01 \quad 00.04 \quad 00.01$ $\begin{array}{lllllllllllll}0.72 & 00.31 & 00.03 & 08.48 & 00.01 & 00.18 & 01.37 & 00.07 & 00.23\end{array}$ $00.00 \quad 00.00 \quad 00.00 \quad 00.00 \quad 00.00 \quad 00.00 \quad 00.00 \quad 00.00 \quad 00.00$ $\begin{array}{lllllllll}03.04 & 04.35 & 02.24 & 00.90 & 02.69 & 03.30 & 03.83 & 07.63 & 02.32\end{array}$
97.0197 .90100 .1096 .4697 .6799 .0999 .4398 .5198 .59

QUART2 ORTHO. ALBIT ANORTH NEPHEL LEUCIT WOLLAS DIOPSI HYPERS OLIVIN ACMITE CORIND ILMENI MAGNET HEMATI APATIT CHROMI

CALCIT

PYRITE H2O T $\begin{array}{llllllllll}39.99 & 34.43 & 00.00 & 28.35 & 00.00 & 13.68 & 00.00 & 16.91 & 08.95\end{array}$ $\begin{array}{lllllllllll}20.70 & 25.88 & 13.51 & 12.88 & 01.21 & 14.69 & 07.67 & 03.83 & 00.54\end{array}$ $03.53 \quad 00.77 \quad 15.97 \quad 05.33 \quad 36.03 \quad 42.91 \quad 30.80 \quad 26.59 \quad 51.98$ $\begin{array}{lllllllllll}02.05 & 14.31 & 19.37 & 02.13 & 19.66 & 08.81 & 19.85 & 16.90 & 14.27\end{array}$ $00.00 \quad 00.00 \quad 00.00 \quad 00.00 \quad 00.00 \quad 00.00 \quad 00.00 \quad 00.00 \quad 00.00$ $\begin{array}{lllllllllll}00.00 & 00.00 & 00.00 & 00.00 & 00.00 & 00.00 & 00.00 & 00.00 & 00.00\end{array}$ $00.00 \quad 00.00 \quad 00.00 \quad 00.00 \quad 00.00 \quad 00.00 \quad 00.00 \quad 00.00 \quad 00.00$ $00.00 \quad 00.00 \quad 26.15 \quad 00.00 \quad 07.54 \quad 00.00 \quad 00.00 \quad 10.48 \quad 00.00$ $\begin{array}{lllllllllll}14.24 & 09.52 & 03.96 & 15.72 & 23.87 & 08.60 & 18.16 & 10.87 & 14.68\end{array}$ $00.00 \quad 00.00 \quad 13.79 \quad 00.00 \quad 01.21 \quad 00.00 \quad 08.35 \quad 00.00 \quad 00.00$ $\begin{array}{llllllllll}00.00 & 00.00 & 00.00 & 00.00 & 00.00 & 00.00 & 00.00 & 00.00 & 00.00\end{array}$ $\begin{array}{lllllllllll}09.85 & 05.51 & 00.00 & 05.97 & 00.00 & 03.24 & 01.86 & 00.00 & 02.25\end{array}$ $\begin{array}{llllllllll}00.79 & 00.87 & 01.31 & 00.82 & 02.89 & 00.88 & 01.25 & 02.33 & 01.07\end{array}$ $\begin{array}{llllllllll}02.82 & 02.87 & 03.17 & 02.67 & 04.42 & 02.86 & 03.10 & 03.98 & 03.02\end{array}$ $00.00 \quad 00.00 \quad 00.00 \quad 00.00 \quad 00.00 \quad 00.00 \quad 00.00 \quad 00.00 \quad 00.00$ $\begin{array}{llllllllll}0 & 0.19 & 00.24 & 00.43 & 00.02 & 00.39 & 00.33 & 00.02 & 00.10 & 00.02\end{array}$ $00.00 \quad 00.00 \quad 00.00 \quad 00.51 \quad 00.00 \quad 00.00 \quad 00.13 \quad 00.00 \quad 00.00$ $00.00 \quad 00.00 \quad 00.00 \quad 00.00 \quad 00.00 \quad 00.00 \quad 00.00 \quad 00.00 \quad 00.00$ $\begin{array}{lllllllllll}02.74 & 01.18 & 00.11 & 24.75 & 00.04 & 00.68 & 05.04 & 00.27 & 00.87\end{array}$ $\begin{array}{llllllllll}03.10 & 04.42 & 02.24 & 00.85 & 02.75 & 03.32 & 03.77 & 07.74 & 02.34\end{array}$

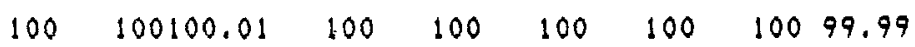

$65.00 \quad 60.90 \quad 51.00 \quad 51.60 \quad 52.20$ $\begin{array}{llll}17.90 & 15.30 \quad 13.30 \quad 10.70 \quad 11.80\end{array}$ $02.06 \quad 02.58 \quad 02.92 \quad 01.92 \quad 03.00$ $01.1203 .08 \quad 05.27 \quad 16.18 \quad 09.09$ $00.59 \quad 06.08 \quad 08.74 \quad 01.48 \quad 06.12$ $02.64 \quad 03.56 \quad 09.30 \quad 04.96 \quad 07.86$ $02.05 \quad 02.58 \quad 01.6101 .33 \quad 04.20$ 04.3801 .7203 .1400 .7900 .46 $00.56 \quad 01.08 \quad 01.42 \quad 00.42 \quad 01.50$ $00.00 \quad 00.00 \quad 00.00 \quad 00.0000 .10$ $00.03 \quad 00.0900 .06 \quad 00.1500 .15$ $\begin{array}{llllll}0 & 0.05 & 00.26 & 00.11 & 00.19 & 00.15\end{array}$ $\begin{array}{lllllll}0 & 0.58 & 00.04 & 00.14 & 06.22 & 00.06\end{array}$ $00.00 \quad 00.00 \quad 00.00 \quad 00.00 \quad 00.00$ $\begin{array}{llllll}02.70 & 02.47 & 02.30 & 06.09 & 02.29\end{array}$

99.6699 .7499 .31102 .0498 .98

$\begin{array}{llllll}31.30 & 21.65 & 00.11 & 21.42 & 00.00\end{array}$ $\begin{array}{lllllll}25.92 & 10.18 & 18.64 & 04,16 & 02.74\end{array}$ $\begin{array}{llllll}17.37 & 21.87 & 13.68 & 10.04 & 35.86\end{array}$ $02.60 \quad 25.13 \quad 19.88 \quad 05.44 \quad 12.10$ $00.00 \quad 00.00 \quad 00.00 \quad 00.00 \quad 00.00$ $00.00 \quad 00.00 \quad 00.00 \quad 00.00 \quad 00.00$ $00.00 \quad 00.00 \quad 00.00 \quad 00.00 \quad 00.00$ $00.00 \quad 02.69 \quad 18.22 \quad 00.00 \quad 14.29$ $06.59 \quad 09.43 \quad 19.40 \quad 24.32 \quad 24.66$ $00.00 \quad 00.00 \quad 00.00 \quad 00.00 \quad 00.03$ $00.00 \quad 00.00 \quad 00.00 \quad 00.00 \quad 00.00$ $\begin{array}{llllll}08.95 & 00.00 & 00.00 & 04.83 & 00.00\end{array}$ $\begin{array}{lllllll}01.07 & 02.05 & 02.71 & 00.71 & 02.87\end{array}$ $\begin{array}{llllll}01.05 & 03.75 & 04,25 & 02.48 & 04.39\end{array}$ $01.34 \quad 00.00 \quad 00.00 \quad 00.00 \quad 00.00$ $\begin{array}{llllll}00.12 & 00.62 & 00.26 & 00.40 & 00.36\end{array}$ $00.00 \quad 00.00 \quad 00.00 \quad 00.00 \quad 00.15$ $00.00 \quad 00.00 \quad 00.00 \quad 00.00 \quad 00.00$ $\begin{array}{lllllll}01.09 & 00.15 & 00.53 & 20.76 & 00.23\end{array}$ $\begin{array}{lllllll}02.70 & 02.47 & 02.31 & 05.43 & 02.31\end{array}$

10099.9999 .9999 .9999 .99 
9468294675921429215292164921539216292161921639215092108

\begin{tabular}{|c|c|c|c|c|c|c|c|c|c|c|c|}
\hline$C H \quad\{p \beta m\}$ & 36 & 47 & 45 & $.25 \%$ & 69 & 150 & 57 & 60 & 80 & 250 & 18 \\
\hline $2 \pi$ & $.56 \%$ & 160 & 140 & 100 & 110 & 100 & 100 & 82 & 150 & $.21 \%$ & 94 \\
\hline $\mathrm{Pb}$ & $<.5$ & $<.5$ & 5 & 5 & $<.5$ & 5.5 & $<.5$ & 5 & $<.5$ & $.10 \%$ & 23 \\
\hline $\mathrm{Ni}$ & 18 & 23 & 120 & & & & 52 & & 19 & 34 & 14 \\
\hline $\mathrm{Co}$ & 9 & 14 & & & & & 17 & & & 10 & 15 \\
\hline $\mathrm{Cr}$ & 33 & 22 & & & & & * & & 17 & & 24 \\
\hline v & 59 & 78 & & & & & & & & & \\
\hline$w$ & 2 & $<2$ & & & & & & & & & \\
\hline Sil & 4 & 8 & & & & & & & & & \\
\hline$A S$ & $.20 \%$ & 30 & & & $<1$ & $<1$ & & & 1.5 & & 8 \\
\hline 110 & $<3$ & $<3$ & & & & & & & & 3 & $\langle 3$ \\
\hline Au $(p p b)$ & $\langle 15$ & $<15$ & $\therefore 15$ & 15 & $\langle 15$ & $<15$ & $<15$ & 815 & 15 & $<15$ & 615 \\
\hline Ag & $<250$ & 250 & 250 & $<250$ & $<250$ & $<250$ & 250 & $<250$ & $<250$ & 700 & $<250$ \\
\hline
\end{tabular}

921679215172166

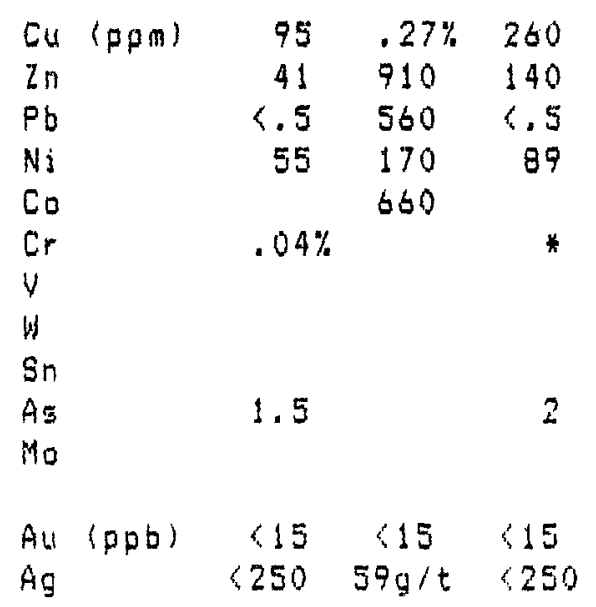


F. - C. DE ROBERGE

9217592174921739215494668

$\begin{array}{llllllll}\text { S102 } & 35.60 & 34.70 & 45.20 & 47.70 & 53.30 \\ \text { AL203 } & 01.89 & 01.44 & 02.79 & 03.47 & 13.00 \\ \text { FE203 } & 01.79 & 01.71 & 01.96 & 02.04 & 02.14 \\ \text { FEO } & 12.77 & 13.70 & 09.31 & 07.16 & 09.32 \\ \text { CAO } & 02.14 & 00.26 & 11.40 & 14.50 & 05.10 \\ M 60 & 32.70 & 34.20 & 21.20 & 19.20 & 02.77 \\ \text { MA20 } & 00.01 & 00.01 & 00.13 & 00.14 & 03.66 \\ K 20 & 00.01 & 00.12 & 00.03 & 00.03 & 01.96 \\ \text { T102 } & 00.29 & 00.21 & 00.46 & 00.54 & 00.34 \\ \text { CR203 } & 00.77 & 00.22 & 00.72 & 00.00 & 00.00 \\ M N 0 & 00.20 & 00.20 & 00.17 & 00.20 & 00.09 \\ \text { P205 } & 00.02 & 00.03 & 00.03 & 00.03 & 00.14 \\ 5 & 00.15 & 00.08 & 00.03 & 00.01 & 01.91 \\ C D 2 & 00.00 & 00.00 & 00.00 & 00.00 & 00.00 \\ H 20 T & 10.55 & 10.82 & 03.85 & 05.70 & 04.10\end{array}$

$99.0997 .76 \quad 97.28 \quad 100.7398 .13$

QUARTI $00.00 \quad 00.00 \quad 00.00 \quad 00.00 \quad 07.37$

ORTHO. $\quad 00.06 \quad 00.72 \quad 00.18 \quad 00.18 \quad 11.44$

ALBIT $\quad 00.09 \quad 00.0901 .1301 .18 \quad 30.59$

ANORTH $05.12 \quad 01.1207 .13 \quad 08.69 \quad 13.09$

NEPHEL

LEUCIT

WOLLAS

DIOPSI

HYPERS

OLIVIN

ACMITE

CORIND

ILMENI

MAGNET

HEHATI

APATIT

CHROMI

CALCIT

FYRITE

H2OT
$00.00 \quad 00.00 \quad 00.00 \quad 00.00 \quad 00.00$

$00.00 \quad 00.00 \quad 00.00 \quad 00.00 \quad 00.00$

$00.00 \quad 00.00 \quad 00.00 \quad 00.00 \quad 00.00$

$04.3400 .00 \quad 40.51 \quad 49.7109 .23$

$\begin{array}{lllllll}08.32 & 10.32 & 22.65 & 20.11 & 12.58\end{array}$

$66.54 \quad 72.14 \quad 19.35 \quad 10.42 \quad 00.00$

$00.00 \quad 00.00 \quad 00.00 \quad 00.00 \quad 00.00$

$00.00 \quad 00.9100 .00 \quad 00.0000 .00$

$00.5500 .41 \quad 00.90 \quad 01.02 \quad 01.20$

02.6102 .5302 .9202 .9403 .06

$00.00 \quad 00.00 \quad 00.00 \quad 00.00 \quad 00.00$

$\begin{array}{lllllll}00.05 & 00.07 & 00.07 & 00.07 & 00.33\end{array}$

$\begin{array}{lllllll}01.14 & 00.33 & 01.09 & 00.00 & 00.00\end{array}$

$00.00 \quad 00.00 \quad 00.00 \quad 00.00 \quad 00.00$

$00.57 \quad 00.31 \quad 00.12 \quad 00.04 \quad 07.06$

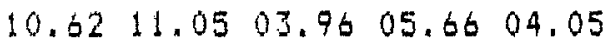

9216592155921569467894683

$51.20 \quad 50.30 \quad 63.70 \quad 50.40 \quad 45.70$ $05.86 \quad 04.20 \quad 15.90 \quad 09.42 \quad 03.92$ $\begin{array}{llllll}02.58 & 02.38 & 02.17 & 02.77 & 01.99\end{array}$ $07.42 \quad 07.40 \quad 02.53 \quad 10.47 \quad 15.49$ $14.50 \quad 14.70 \quad 01.91 \quad 10.80 \quad 10.45$ $\begin{array}{llllll}12.40 & 18.40 & 03.20 & 07.08 & 13.60\end{array}$ $\begin{array}{llllll}01.26 & 00.50 & 05.40 & 02.69 & 00.33\end{array}$ $\begin{array}{lllllll}00.31 & 00.22 & 01.34 & 00.69 & 00.04\end{array}$ $\begin{array}{llllll}01.06 & 00.88 & 00.67 & 01.27 & 00.49\end{array}$ $\begin{array}{llllll}00.07 & 00.22 & 00.00 & 00.00 & 00.00\end{array}$ $\begin{array}{llllll}00.19 & 00.29 & 00.07 & 00.14 & 00.11\end{array}$ $\begin{array}{llllll}00.05 & 00.05 & 00.16 & 00.06 & 00.04\end{array}$ $\begin{array}{llllll}00.08 & 00.03 & 00.18 & 00.06 & 01.02\end{array}$ $00.00 \quad 00.00 \quad 00.00 \quad 00.00 \quad 00.00$ $\begin{array}{llllll}01.13 & 02.23 & 02.78 & 00.97 & 01.81\end{array}$

$98.06 \quad 101.79 \quad 100.096 .8294 .89$

$\begin{array}{lllll}02.25 & 00.00 & 18.01 & 00.78 & 00.00 \\ 01.87 & 01.28 & 07.89 & 04.21 & 00.24 \\ 10.85 & 04.15 & 45.55 & 23.48 & 02.89 \\ 09.59 & 08.41 & 08.40 & 11.96 & 09.14 \\ 00.00 & 00.00 & 00.00 & 00.00 & 00.00 \\ 00.00 & 00.00 & 00.00 & 00.00 & 00.00 \\ 00.00 & 00.00 & 00.00 & 00.00 & 00.00 \\ 50.69 & 49.99 & 00.00 & 35.32 & 36.23 \\ 17.25 & 20.66 & 09.44 & 16.24 & 39.76 \\ 00.00 & 07.75 & 00.00 & 00.00 & 01.85 \\ 00.00 & 00.00 & 00.00 & 00.00 & 00.00 \\ 00.00 & 00.00 & 02.47 & 00.00 & 00.00 \\ 02.05 & 01.64 & 01.27 & 02.49 & 00.96 \\ 03.78 & 03.39 & 03.14 & 04.14 & 02.99 \\ 00.00 & 00.00 & 00.00 & 00.00 & 00.00 \\ 00.12 & 00.12 & 00.38 & 00.15 & 00.10 \\ 00.10 & 00.32 & 00.00 & 00.00 & 00.00 \\ 00.00 & 00.00 & 00.00 & 00.00 & 00.00 \\ 00.30 & 00.11 & 00.67 & 00.23 & 03.95 \\ 01.15 & 02.19 & 02.77 & 01.00 & 01.97 \\ 100100.01 & 99.99 & 100 & 09.98\end{array}$


$9217592174921739215494668 \quad 9216592155921569467894683$

\begin{tabular}{|c|c|c|c|c|c|c|c|c|c|c|c|}
\hline Eu & $(p p \operatorname{s})$ & 10 & 28 & 17 & 110 & 480 & 150 & 11 & 200 & 280 & $1.9 \%$ \\
\hline $2 n$ & & 110 & 110 & 110 & 90 & 100 & 94 & 190 & 82 & 73 & 170 \\
\hline$P b$ & & 4.5 & 4.5 & $<.5$ & 8 & $<.5$ & 1 & $\therefore .5$ & 2 & $<.5$ & $<.5$ \\
\hline $\mathrm{Ni}$ & & $.21 \%$ & $.14 \%$ & 750 & 340 & 45 & 260 & 490 & 12 & 110 & 190 \\
\hline Co & & 110 & 110 & & 56 & 310 & & & & 53 & 67 \\
\hline Cr & & * & * & * & 850 & 35 & * & * & & 22 & 63 \\
\hline$v$ & & 13 & 1.5 & 35 & & 97 & & & & 290 & 140 \\
\hline$W$ & & & & & & $<2$ & & & & $<2$ & $<2$ \\
\hline$s \pi$ & & & & & & 1 & & & & 2 & 7 \\
\hline$A=$ & & & & & & 49 & 2 & & & 7 & $\langle 1$ \\
\hline 110 & & & & & & $<3$ & & & & $\{3$ & 43 \\
\hline AL & $(p p b)$ & & & & $\begin{array}{r}15 \\
250\end{array}$ & 65 & $\begin{array}{r}<5 \\
250\end{array}$ & <15 & $\langle 15$ & 415 & $\begin{array}{l}740 \\
=\end{array}$ \\
\hline
\end{tabular}


F. - C. DE BOURBEAU

94677946769214692141921449214592159921589467392145

\begin{tabular}{|c|c|c|c|c|c|c|c|c|c|c|}
\hline & 7.00 & 46.00 & 40,20 & 49.00 & 40.60 & 47,61 & 52.00 & 44.70 & 45.70 & \\
\hline & 30 & 09.85 & 04.87 & 12.60 & 07.39 & 14.70 & 14.80 & 20.80 & 02.58 & 05.64 \\
\hline & .65 & 02.49 & 01.96 & 02.17 & 02.15 & 02.15 & 02.98 & 02.35 & 02.03 & 02.15 \\
\hline$?$ & 93 & 11.26 & 13.81 & 06.74 & 12.55 & 05.53 & 07.67 & 07.71 & 09.87 & 10.21 \\
\hline t & 37 & 08.66 & 02.35 & 08.82 & 06.10 & 10.80 & 05.00 & 04.90 & 19.40 & 10.10 \\
\hline$i c$ & 40 & 14.70 & 27 & 10.80 & 21.90 & 10.40 & 06.38 & 05.86 & 13.50 & 16.70 \\
\hline 20 & & 01.00 & 00.18 & 01,02 & 00.55 & 02.70 & 04.24 & 03.88 & 09 & 74. \\
\hline 20 & 00.87 & 00.64 & 00.03 & 04.30 & 00.07 & 00.65 & 00.39 & 01.44 & 00.04 & 00.09 \\
\hline 102 & 01.15 & 00.99 & 00.46 & 00.67 & 00.65 & 00.65 & 01.48 & 00.83 & 00.53 & 00.65 \\
\hline 203 & 00.0 & 00.12 & 00.31 & 00.00 & 00.28 & 00.00 & 00.00 & 00.00 & 00.16 & 00.00 \\
\hline & 00.18 & 00.20 & 00.21 & 00.14 & 00.24 & 00.17 & 00.18 & 00.14 & 00.73 & 00.19 \\
\hline 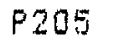 & 00.09 & 00.10 & 00.06 & 00.17 & 00.07 & 00.04 & 00.15 & 00.09 & 00.03 & 00.01 \\
\hline & 00.02 & 00.02 & 00.05 & 00.03 & 00.08 & 00.23 & 00.02 & 00,03 & 01.46 & 01.15 \\
\hline 16 & 00.00 & 00.00 & 00.00 & 00.00 & 00.00 & 00.00 & 00.00 & 00.00 & 00.00 & 00.00 \\
\hline 60 & 03.09 & 03.29 & 07.94 & 02.58 & 05.78 & 02.77 & 03.20 & 05.57 & 01.28 & 02.42 \\
\hline & & & & 17. & 70. & 78.4 & 70.49 & 98,28 & 7.4 & \\
\hline
\end{tabular}

QUARTZ $00.00 \quad 00.00 \quad 00.00 \quad 00.00 \quad 00.00 \quad 00.00 \quad 02.56 \quad 00.00 \quad 00.00 \quad 00.00$

ORTHO. $\quad 05.26 \quad 03.81 \quad 00.18 \quad 25.64 \quad 00.42 \quad 03.8902 .34 \quad 08.65 \quad 00.24 \quad 00.52$

$\begin{array}{lllllllllllll}\text { ALBIT } & 14.27 & 08.52 & 01.52 & 08.71 & 04.72 & 23.03 & 36.41 & 32.26 & 00.76 & 11.11\end{array}$

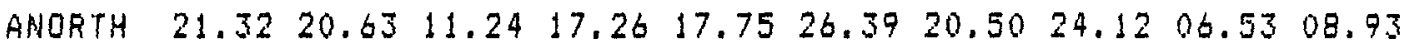

NEPHEL $\quad 00.00 \quad 00.00 \quad 00.00 \quad 00.00 \quad 00.00 \quad 00.05 \quad 00.00 \quad 00.61 \quad 00.00 \quad 00.00$

LEUCIT $00.00 \quad 00.00 \quad 00.00 \quad 00.00 \quad 00.00 \quad 00.00 \quad 00.00 \quad 00.00 \quad 00.00 \quad 00.00$

WOLLAS $00.00 \quad 00.00 \quad 00.00 \quad 00.00 \quad 00.00 \quad 00.00 \quad 00.00 \quad 00.00 \quad 00.00 \quad 00.00$

DIDPSI $12.56 \quad 17.74 \quad 00.00 \quad 20.69 \quad 10.04 \quad 22.01 \quad 02.99 \quad 00.00 \quad 72.26 \quad 32.15$

HYPERS $29.86 \quad 25.29 \quad 29.42 \quad 02.52 \quad 16.90 \quad 00.00 \quad 24.2800 .00 \quad 00.02 \quad 29.83$

QLIVIN $07.01 \quad 14.69 \quad 44.81 \quad 17.60 \quad 39.00 \quad 16.4400 .00 \quad 19.09 \quad 09.1606 .58$

ACMITE $00.00 \quad 00.00 \quad 00.00 \quad 00.00 \quad 00.00 \quad 00.00 \quad 00.00 \quad 00.00 \quad 00.00 \quad 00.00$

CORIND $00.00 \quad 00.00 \quad 00.41 \quad 00.00 \quad 00.00 \quad 00.00 \quad 00.00 \quad 04.2400 .00 \quad 00.00$

$\begin{array}{llllllllllll}\text { ILMENI } & 02.23 & 01.89 & 00.87 & 01.28 & 01.25 & 01.25 & 02.85 & 01.60 & 01.01 & 01.21\end{array}$

MAGNET $03.93 \quad 03.63 \quad 02.84 \quad 03.17 \quad 03.16 \quad 03.16 \quad 04.3903 .44 \quad 02.9503 .06$

HEMATI $00.00 \quad 00.00 \quad 00.00 \quad 00.00 \quad 00.00 \quad 00.00 \quad 00.00 \quad 00.00 \quad 00.00 \quad 00.00$

APATIT $\quad 00.22 \quad 00.24 \quad 00.14 \quad 00.4100 .17 \quad 00.10 \quad 00.36 \quad 00.22 \quad 00.07 \quad 00.02$

CHROMI $00.11 \quad 00.18 \quad 00.46 \quad 00.00 \quad 00.42 \quad 00.00 \quad 00.00 \quad 00.00 \quad 00.24 \quad 00.00$

CALCIT $00.00 \quad 00.00 \quad 00.00 \quad 00.00 \quad 00.00 \quad 00.00 \quad 00.00 \quad 00.00 \quad 00.00 \quad 00.00$

PYFITE $\quad 00.08 \quad 00.08 \quad 00.1900 .1100 .30 \quad 00.87 \quad 00.08 \quad 00.1105 .4804 .22$

$\begin{array}{lllllllllllll}H 20 & 03.16 & 03.31 & 07.92 & 02.60 & 05.86 & 02.80 & 03.25 & 05.66 & 01.28 & 02.37\end{array}$

$100.01100 .01 \quad 10099.9999 .9999 .99100 .01 \quad 100 \quad 100 \quad 100$ 
FILON-COUCHE DE BOUREEAU

94677946769214692141921449214592159921589467392143

\begin{tabular}{|c|c|c|c|c|c|c|c|c|c|c|}
\hline Cu (ppm) & 24 & 38 & 31 & 56 & 27 & 52 & 47 & 29 & 490 & 19 \\
\hline $2 n$ & 80 & 120 & 140 & 150 & 190 & 79 & 120 & 110 & 870 & 100 \\
\hline $\mathrm{Pb}$ & $<.5$ & $\therefore 5$ & $<.5$ & 2 & $<.5$ & $<.5$ & $<.5$ & $<.5$ & 370 & $<.5$ \\
\hline $\mathrm{Ni}$ & 230 & 310 & 810 & 120 & 570 & 82 & 62 & & $\$ 10$ & 120 \\
\hline Co & 61 & 69 & & & & & & & 86 & 34 \\
\hline $\mathrm{Cr}$ & * & * & * & 950 & * & 98 & & & * & 930 \\
\hline V & 220 & 200 & & & & & & & 200 & \\
\hline$W$ & $\langle 2$ & $<2$ & & & & & & & $<2$ & \\
\hline$s_{n}$ & 1 & 3 & & & & & & & 5 & \\
\hline$A \Xi$ & 8 & 160 & & & & & & & 225 & \\
\hline No & $<3$ & $<3$ & & & & & & & 43 & \\
\hline Au $(p p Q)$ & 415 & $\langle 15$ & $<15$ & $\langle 15$ & 415 & $<15$ & 415 & $<15$ & 15 & $<15$ \\
\hline $\mathrm{Ag}$ & $<250$ & $<250$ & $<250$ & 2250 & $<250$ & $<250$ & $<250$ & $<250$ & $4 g / t$ & $<250$ \\
\hline
\end{tabular}




$\begin{array}{llllllll}\text { SIO2 } & 49.20 & 60.30 & 65.60 & 51.30 & 82.10 & 95.00 & 47.60 \\ \text { AL2OS } & 13.00 & 15.10 & 17.10 & 17.70 & 03.78 & 00.52 & 10.80 \\ \text { FE2O3 } & 02.88 & 02.12 & 01.85 & 02.16 & 01.58 & 01.54 & 02.26 \\ \text { FEO } & 09.29 & 06.19 & 01.19 & 05.89 & 03.44 & 9.258 & 07.79 \\ \text { CAO } & 12.00 & 06.17 & 03.15 & 06.48 & 00.11 & 00.06 & 08.10 \\ \text { MGO } & 05.13 & 01.63 & 01.92 & 03.67 & 00.43 & 00.15 & 13.10 \\ \text { NA2O } & 03.03 & 05.46 & 05.44 & 03.92 & 00.06 & 00.03 & 02.37 \\ \text { K20 } & 00.08 & 00.54 & 02.19 & 03.35 & 01.39 & 00.20 & 00.66 \\ \text { T102 } & 01.38 & 00.62 & 00.35 & 00.66 & 00.08 & 00.04 & 00.76 \\ \text { CR203 } & 00.00 & 00.00 & 00.00 & 00.00 & 00.00 & 00.00 & 00.16 \\ \text { MNO } & 00.12 & 00.08 & 00.03 & 00.11 & 00.02 & 00.01 & 00.15 \\ \text { F205 } & 00.07 & 00.14 & 00.16 & 00.34 & 00.01 & 00.01 & 00.35 \\ \text { S } & 00.02 & 00.02 & 00.05 & 00.07 & 04.32 & 00.14 & 00.02 \\ \text { CO2 } & 00.00 & 00.00 & 00.00 & 00.00 & 00.00 & 00.00 & 00.00 \\ \text { H2D T } & 01.33 & 01.34 & 01.72 & 01.55 & 03.40 & 00.14 & 04.10\end{array}$

$97.5399 .71 \quad 100.7597 .2 \quad 100.7297 .09$

98.21

$\begin{array}{llllllll}\text { QUARTI } & 00.48 & 10.44 & 16.27 & 00.00 & 74.49 & 98.30 & 00.00 \\ \text { ORTHO. } & 00.48 & 03.20 & 12.83 & 20.34 & 08.18 & 01.24 & 03.97 \\ \text { ALBIT } & 26.28 & 46.31 & 45.65 & 32.25 & 00.51 & 00.27 & 20.41 \\ \text { ANQRTH } & 22.17 & 15.14 & 14.46 & 21.38 & 00.48 & 00.24 & 17.18 \\ \text { NEPHEL } & 00.00 & 00.00 & 00.00 & 00.79 & 00.00 & 00.00 & 00.00 \\ \text { LEUCIT } & 00.00 & 00.00 & 00.00 & 00.00 & 00.00 & 00.00 & 00.00 \\ \text { WOLLAS } & 00.00 & 00.00 & 00.00 & 00.00 & 00.00 & 00.00 & 00.00 \\ \text { DIOPSI } & 31.73 & 12.42 & 00.00 & 07.72 & 00.00 & 00.00 & 17.15 \\ \text { HYPERS } & 10.28 & 06.48 & 04.77 & 00.00 & 02.86 & 00.39 & 17.63 \\ \text { OLIVIN } & 00.00 & 00.00 & 00.00 & 10.13 & 00.00 & 00.00 & 13.52 \\ \text { ACMITE } & 00.00 & 00.00 & 00.00 & 00.00 & 00.00 & 00.00 & 00.00 \\ \text { CORIND } & 00.00 & 00.00 & 00.43 & 00.00 & 01.99 & 00.18 & 00.00 \\ \text { ILMENI } & 02.69 & 01.18 & 00.66 & 01.29 & 00.15 & 00.00 & 01.47 \\ \text { MAGNET } & 04.28 & 03.08 & 02.66 & 03.22 & 02.28 & 00.00 & 03.33 \\ \text { HEMATI } & 00.00 & 00.00 & 00.00 & 00.00 & 00.00 & 01.62 & 00.00 \\ \text { APATIT } & 00.17 & 00.33 & 00.38 & 00.83 & 00.02 & 00.02 & 00.84 \\ \text { CHROMI } & 00.00 & 00.00 & 00.00 & 00.00 & 00.00 & 7.597 & 00.24 \\ \text { CALCIT } & 00.00 & 00.00 & 00.00 & 00.00 & 00.00 & 00.00 & 00.00 \\ \text { PYRITE } & 00.08 & 00.08 & 00.19 & 00.27 & 05.63 & 00.00 & 00.09 \\ \text { HZOT } & 01.36 & 01.34 & 01.71 & 01.59 & 03.39 & 00.15 & 04.17 \\ & & & & & & & \\ & 100 & 100100.01100 .01 & 99.98 & 100 & 99.99\end{array}$




\section{STOCK TONALITIQUE DU DYKE LAC LINE \\ $94671946879217094669 \quad 94666$}

\begin{tabular}{|c|c|c|c|c|c|c|}
\hline Cu & (ppon) & 17 & 9 & 7 & 96 & 85 \\
\hline In & & 110 & 61 & 310 & 74 & 130 \\
\hline Fb & & $<.5$ & $<.5$ & $<.5$ & $<.5$ & $<.5$ \\
\hline $\mathrm{Ni}$ & & 90 & 38 & 28 & 12 & 420 \\
\hline Co & & 34 & 23 & 14 & 32 & 48 \\
\hline $\mathrm{Cr}$ & & 24 & 28 & 11 & 3 & * \\
\hline V & & 210 & 78 & 97 & 150 & 170 \\
\hline$\omega$ & - & $<2$ & $<2$ & 42 & $<2$ & $<2$ \\
\hline $\sin$ & & 8 & 3 & 3 & 2 & 4 \\
\hline$A s$ & & 475 & $<1$ & 22 & 22 & 600 \\
\hline Mo & & $<3$ & $<3$ & 43 & $<3$ & $<3$ \\
\hline$A u$ & $(p p b)$ & 415 & 415 & $<15$ & 415 & <15 \\
\hline AO & & $<250$ & $<250$ & $\langle 250$ & $<250$ & $<250$ \\
\hline
\end{tabular}




\section{LEGENDE:}

\section{PROTÉROZOÏQUE}

P1

P2

Conglomérat

P3

Arkose

Grauwacke

Archeen

$2 T$

Tonalite

COMPLEXE DE CUMMINGS: filons-couches de Bourbeau(B). de Ventures( $V$ ), et de Roberge(R)

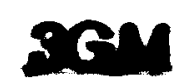

3GL

$3 G$,

$3 G$

4P

D

V6

V7

V9

$\checkmark 10$

$x$

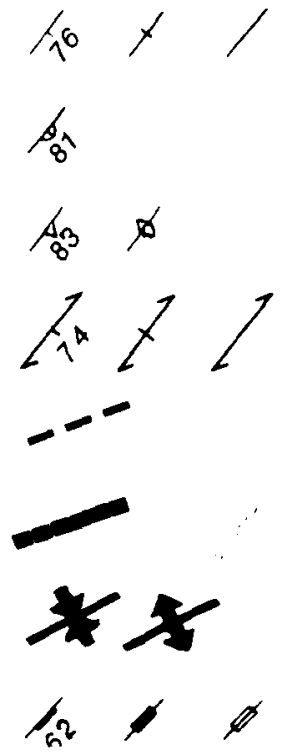

\section{Ferrogabbro}

Leucogabbro

Granophyre

Gabbro

Péridotite

Dunite

\section{VOLCANITES}

Andésite

Basalte

Sédiments et tufs

Agglomérat

SIGNES CONVENTIONNELS

Affleurement: a) isolé, b) aire d'affleurements.

Stratification à sommet non detérminé avec pendage:

a)incliné b) vertical, c)non déterminé

Stratification à sommet déterminé avec pendage

Litage magmatique avec pendage :ajincliné, b)vertical

Schistosité avec pendage: a)incliné, b)vertical, c)non déterminé

Contact géologique présumé

$S$ Faille présumée Cisaillement Zone intensément cisaillée

Trace axiale présumée: a)d'un synforme, b) d'un antiforme

Veine: alincliná hlvortinsla lnint 

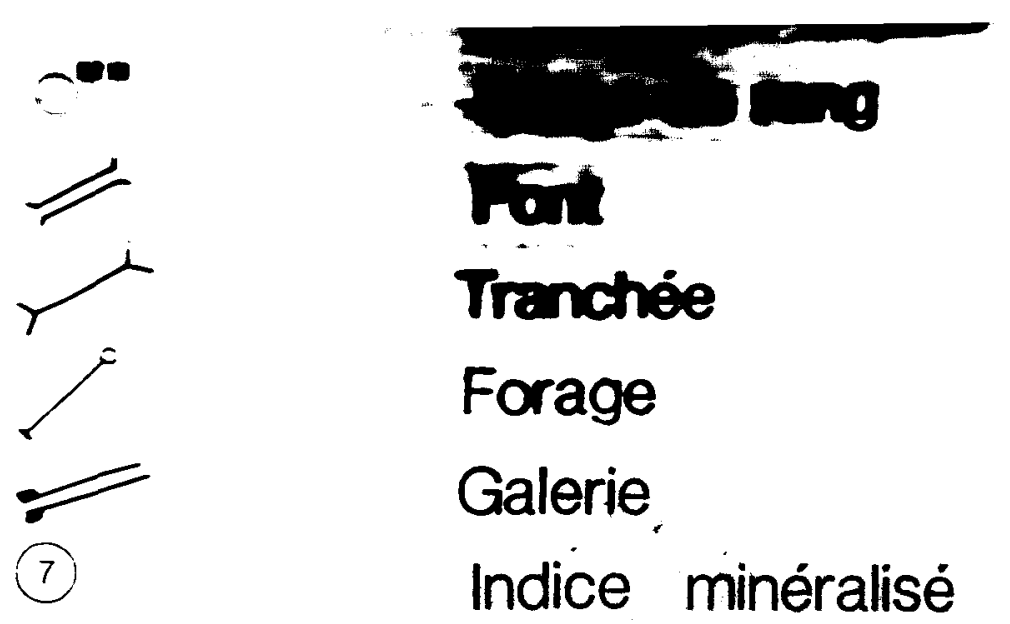

\section{Forage}

Galerie

Indice minéralisé signalé dans le texte

COMPOSITION ET ALTERATION DE LA ROCHE

$\begin{array}{cl}\alpha & \text { Roche felsique } \\ \beta & \text { Roche mafique } \\ \sigma & \text { Silicification } \\ \varnothing & \text { Chloriti } \\ \eta & \text { Carbonatisation } \\ \chi & \text { Serpentinisation } \\ & \text { MINÉRAUX }\end{array}$

$\begin{array}{ll}\text { Py } & \text { Pyrite } \\ \text { Cp } & \text { Chalcopyrite } \\ \text { Mt } & \text { Magnétite } \\ \text { Sph } & \text { Sphalérite } \\ \text { Po } & \text { Pyrrhotine } \\ J & \text { Carbonate } \\ \mathbf{q} & \text { Quartz } \\ \text { C } & \text { Chloritisation } \\ & \\ & \quad \text { TEXTURES } \\ \square & \text { Porphyrique } \\ \text { D } & \text { Coussinée } \\ \Delta & \text { Bréchiforme } \\ * & \text { Variolaire }\end{array}$




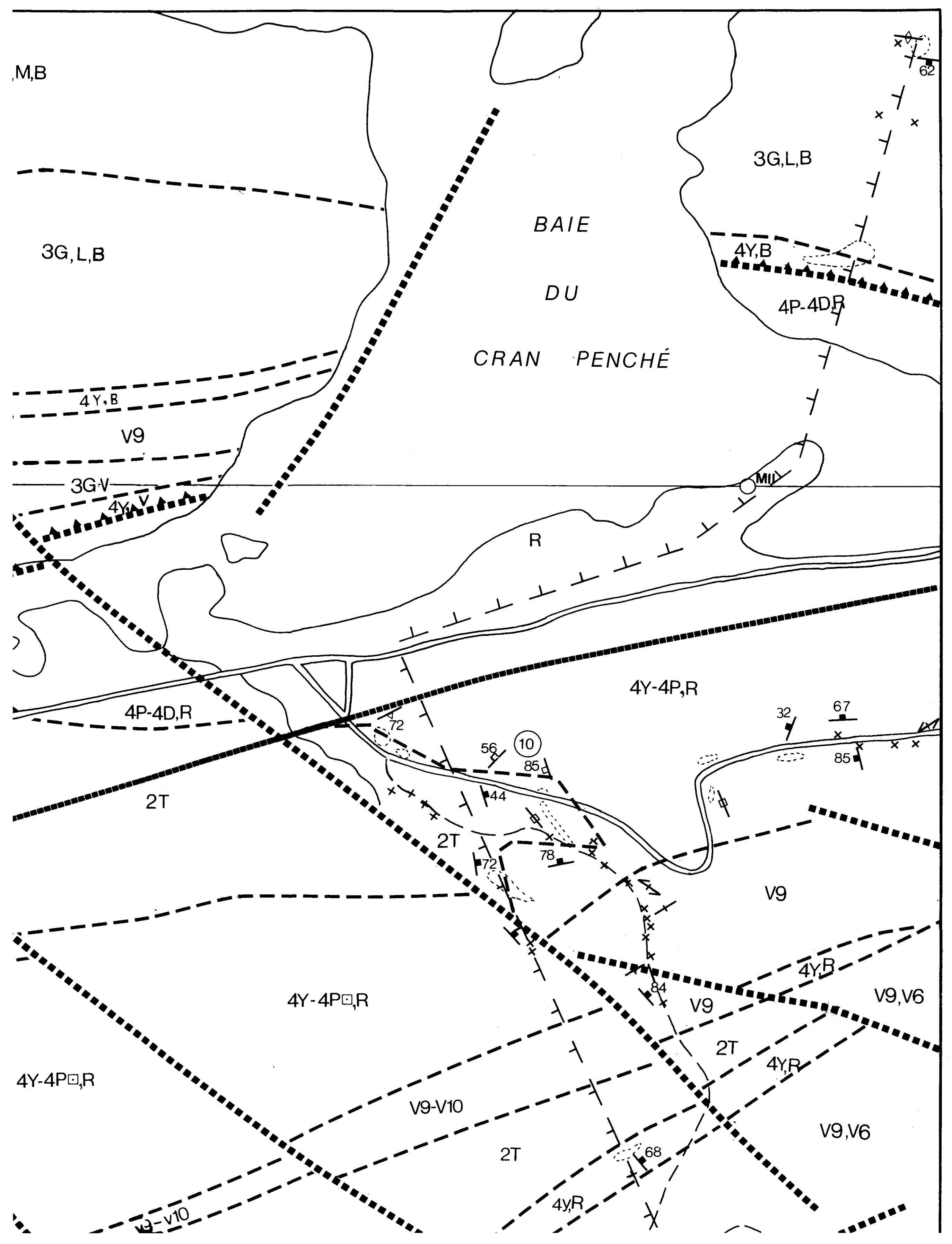




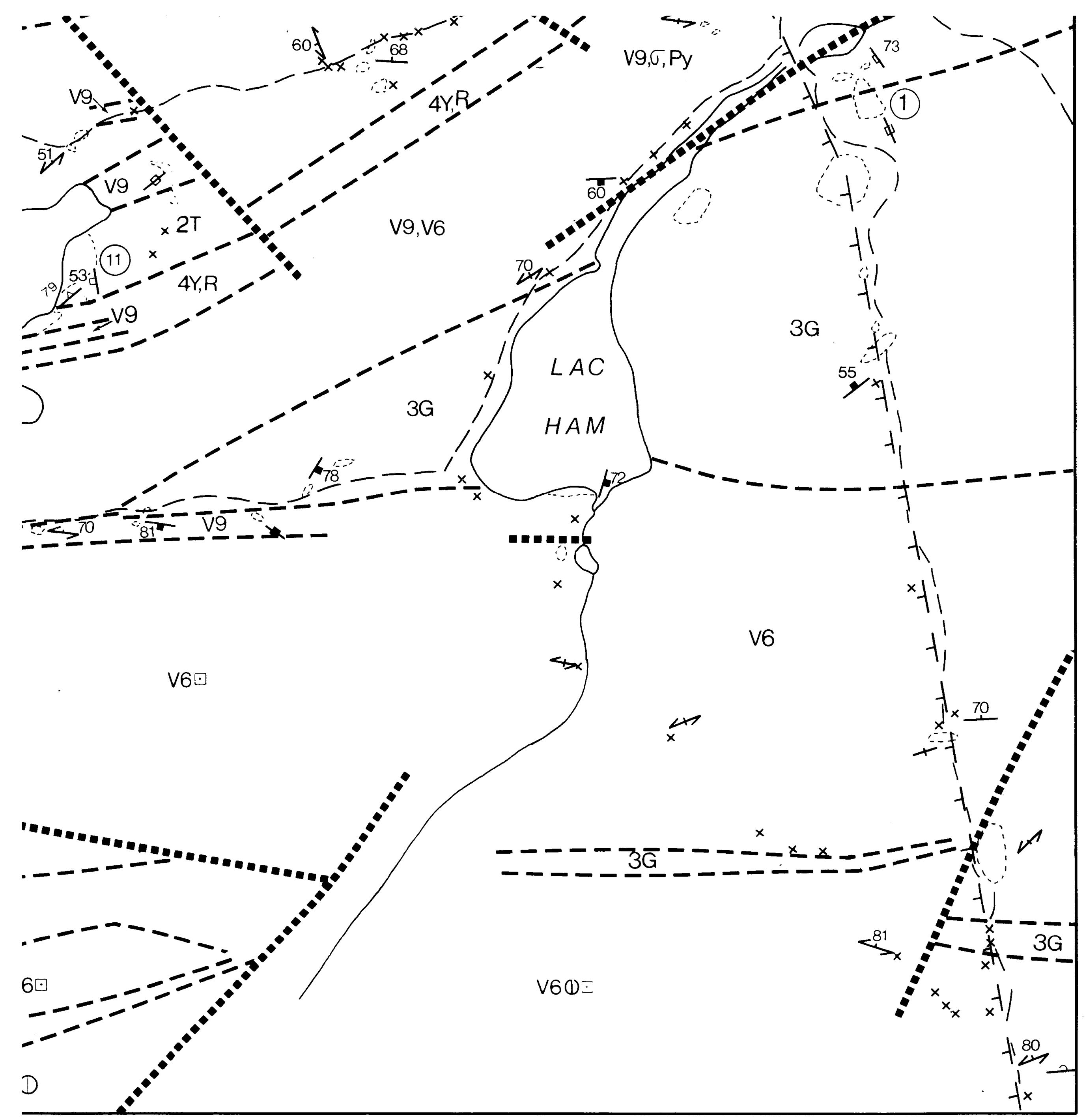




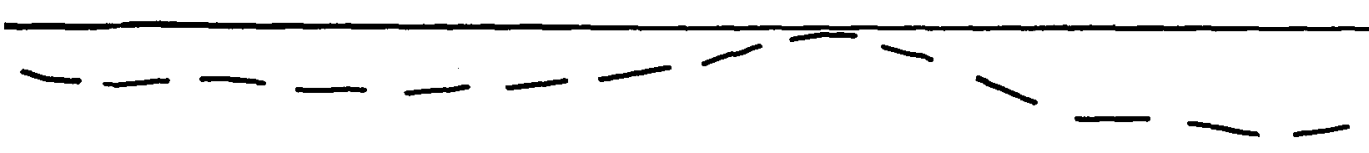

3G,M,B

$x \quad \therefore=x \times x \quad x$

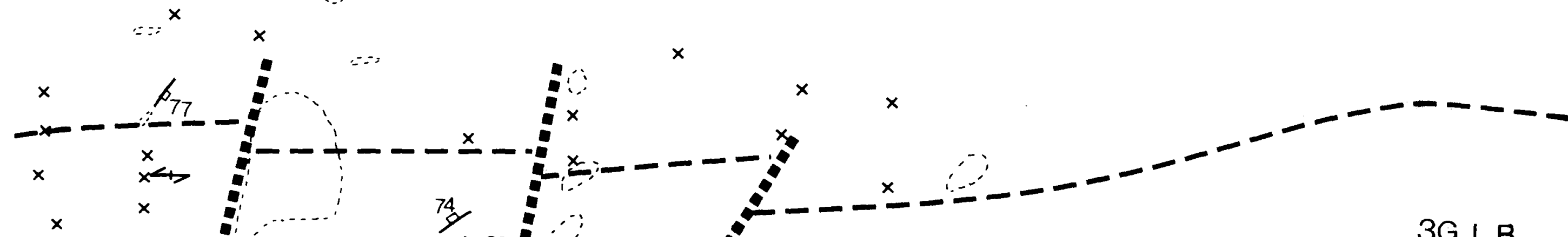

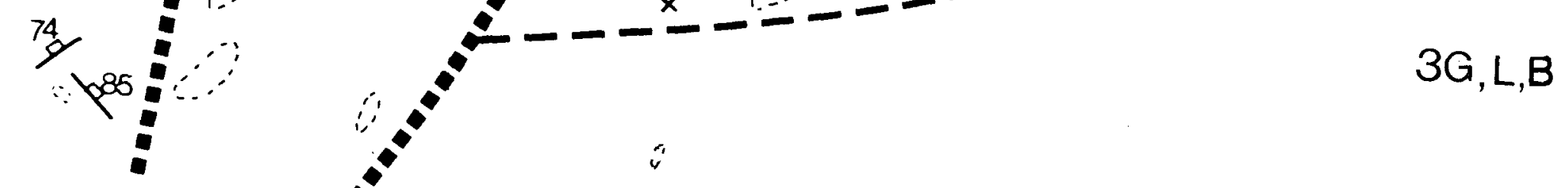

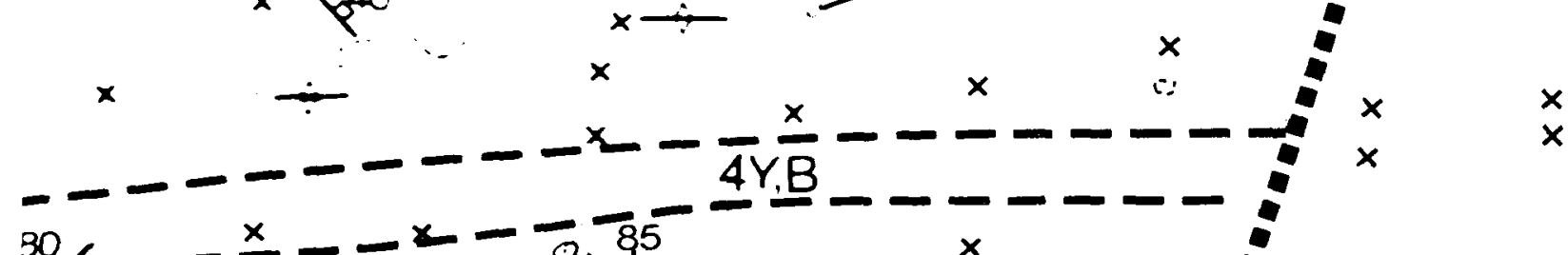

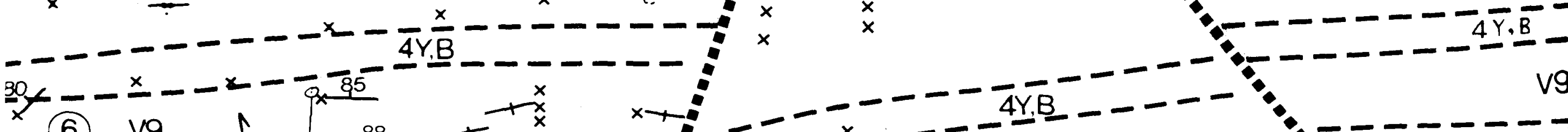

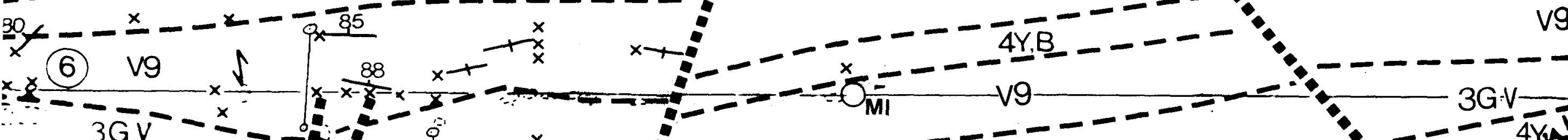

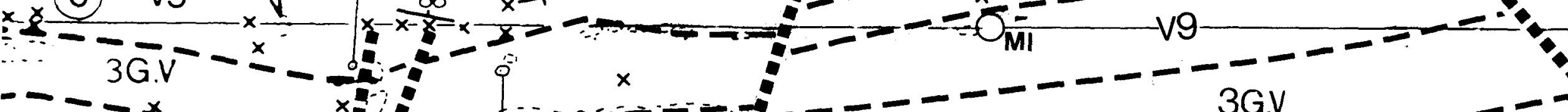

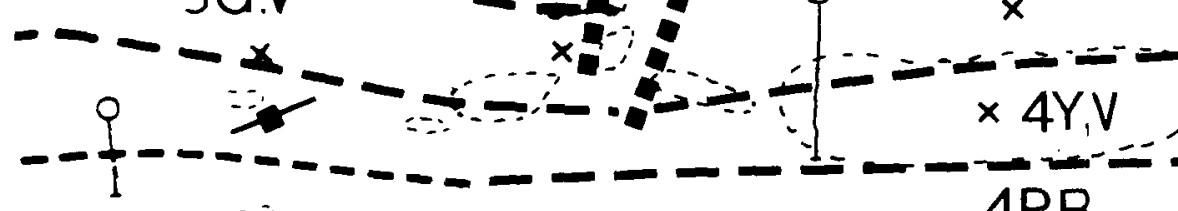

$4 \mathrm{P}, \mathrm{R}$

- $3 \mathrm{G}, \mathrm{V}$

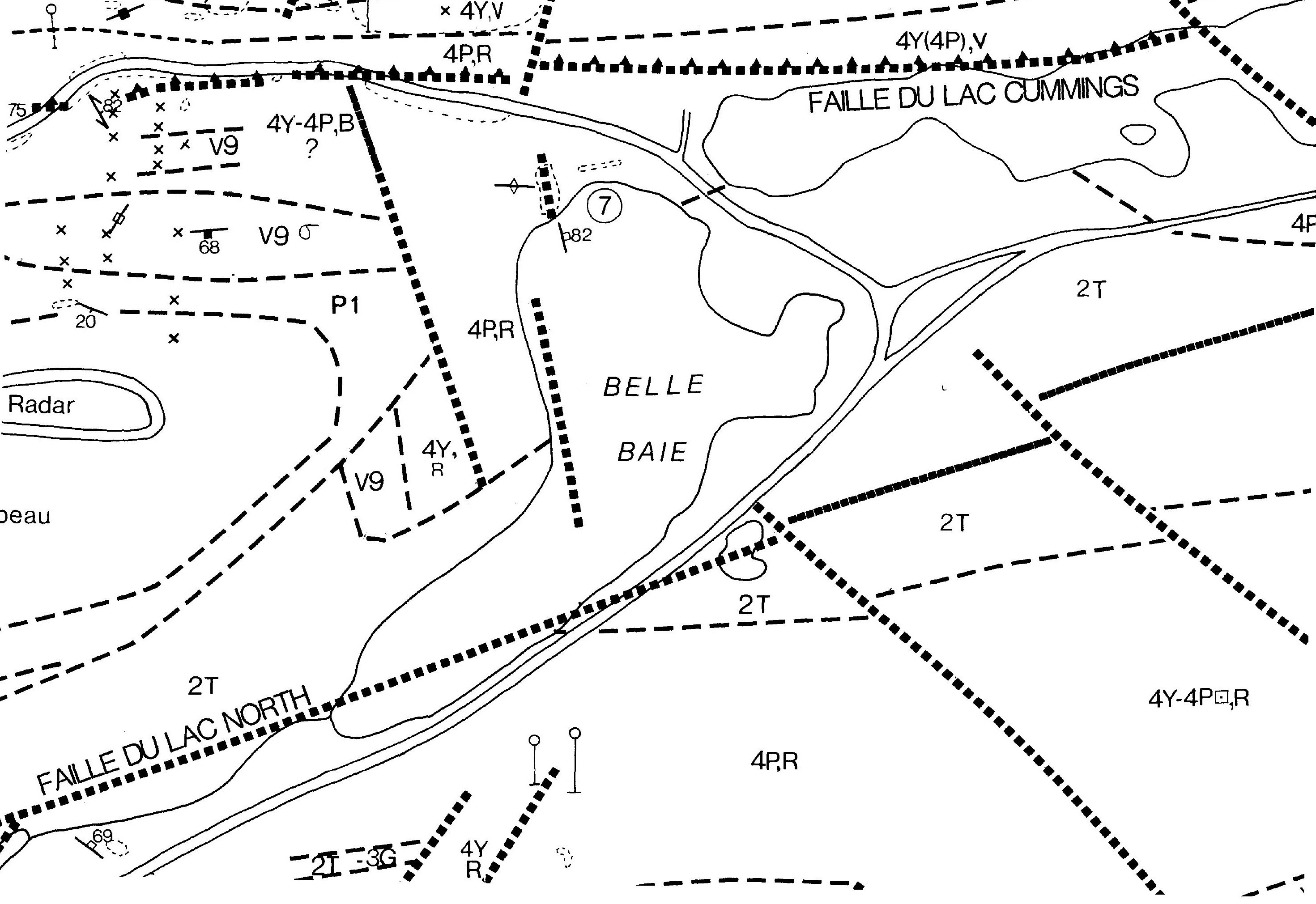




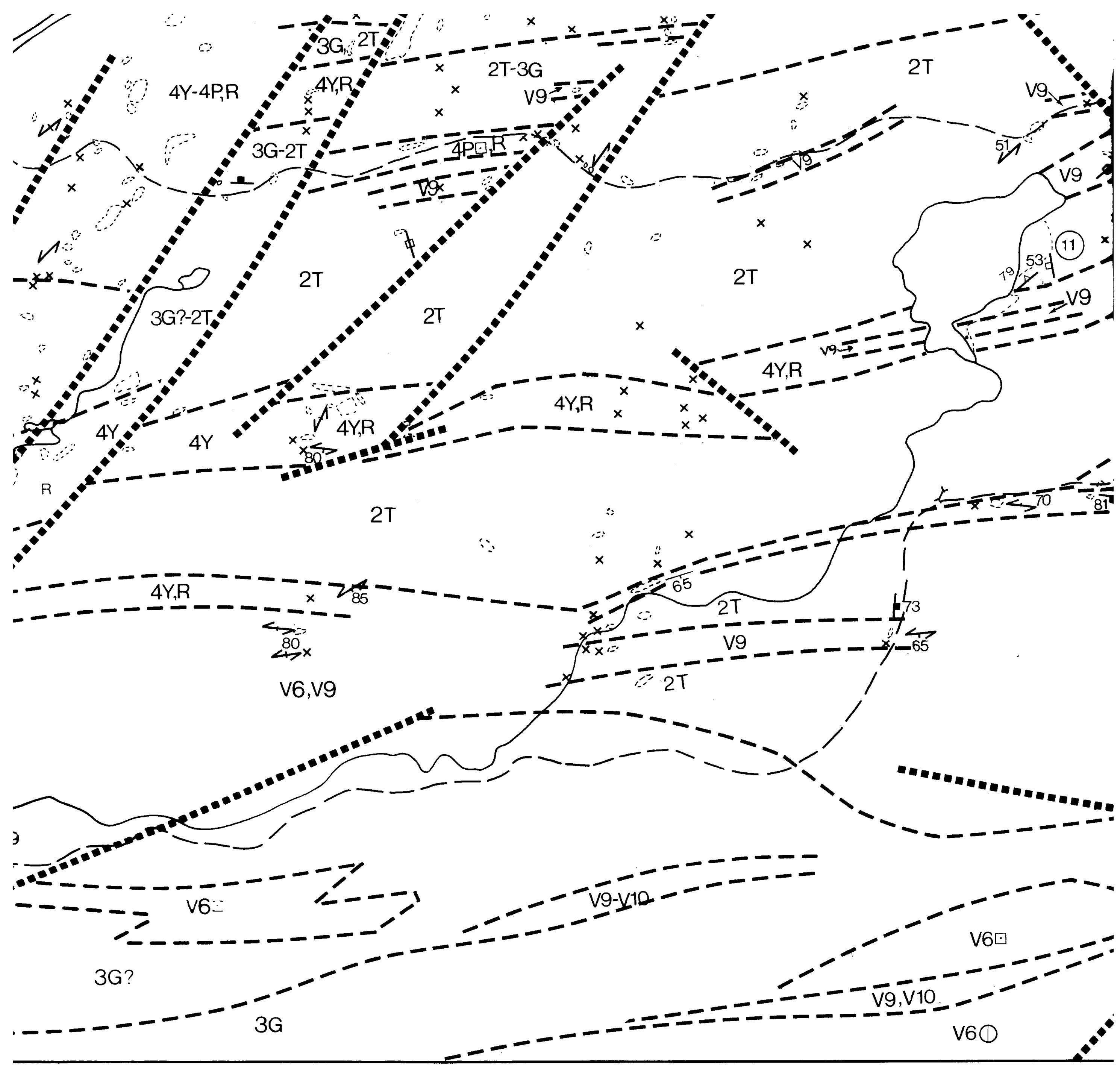




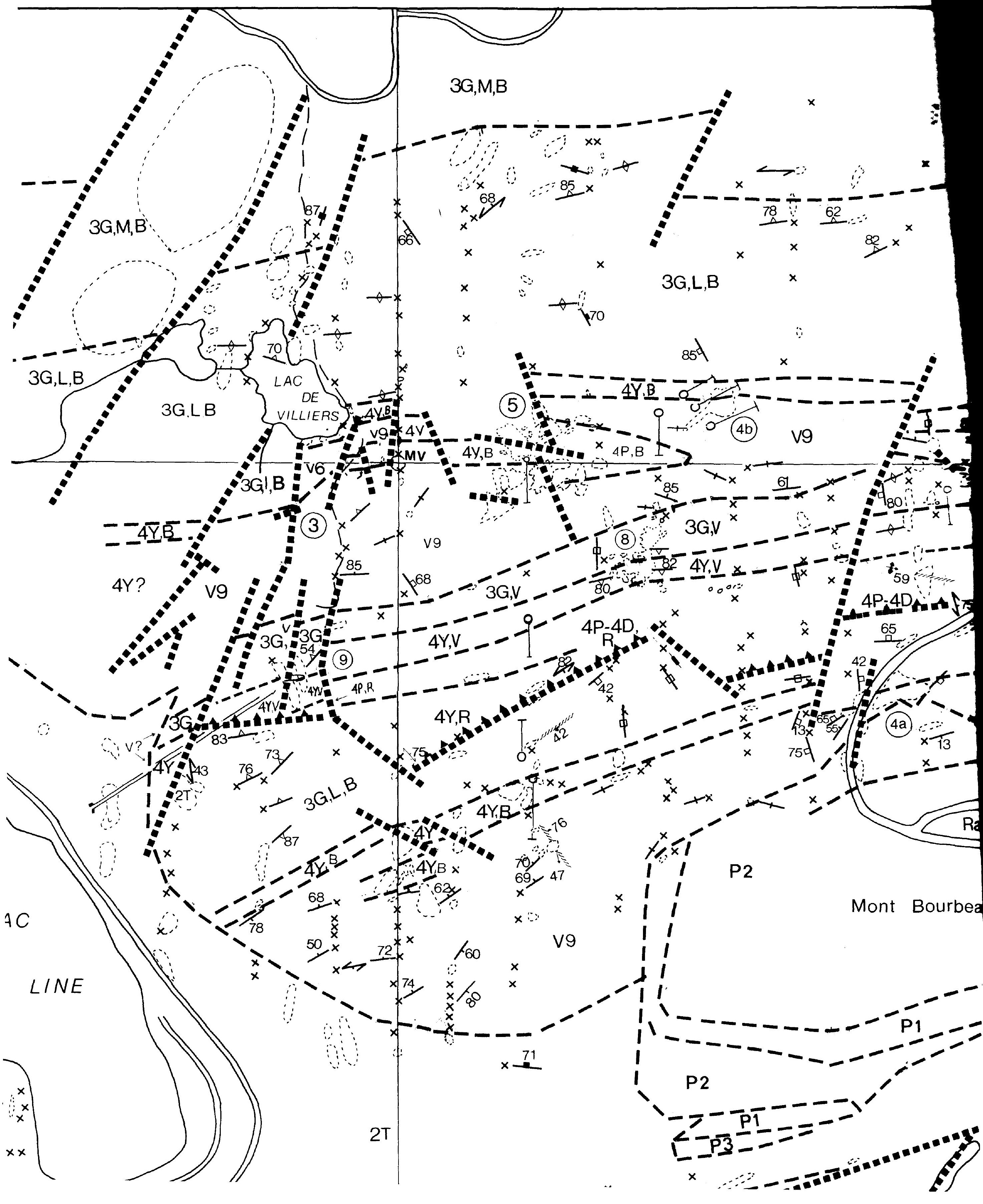




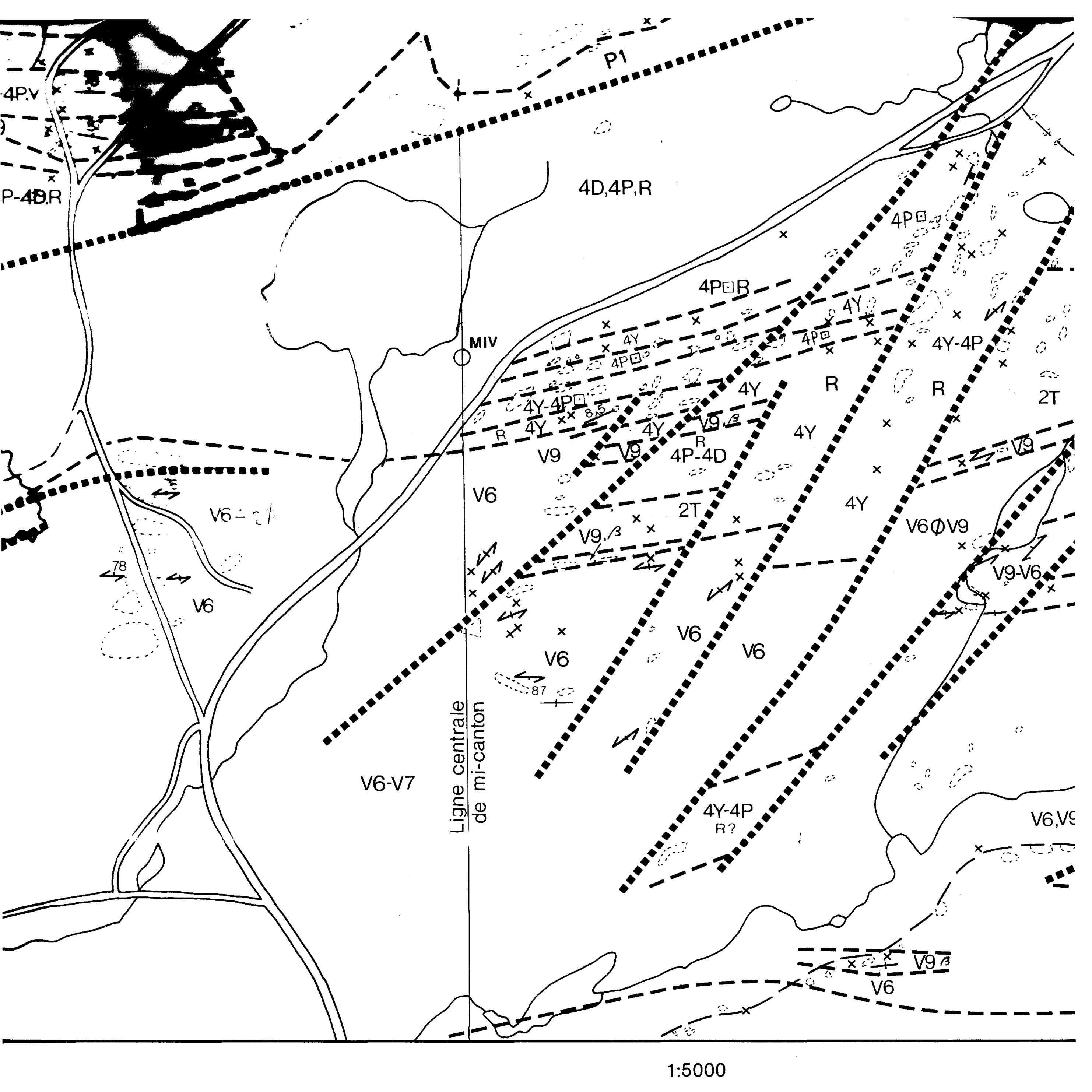

CHIBOUGAMAU

CANTON DE MCKENZIE 


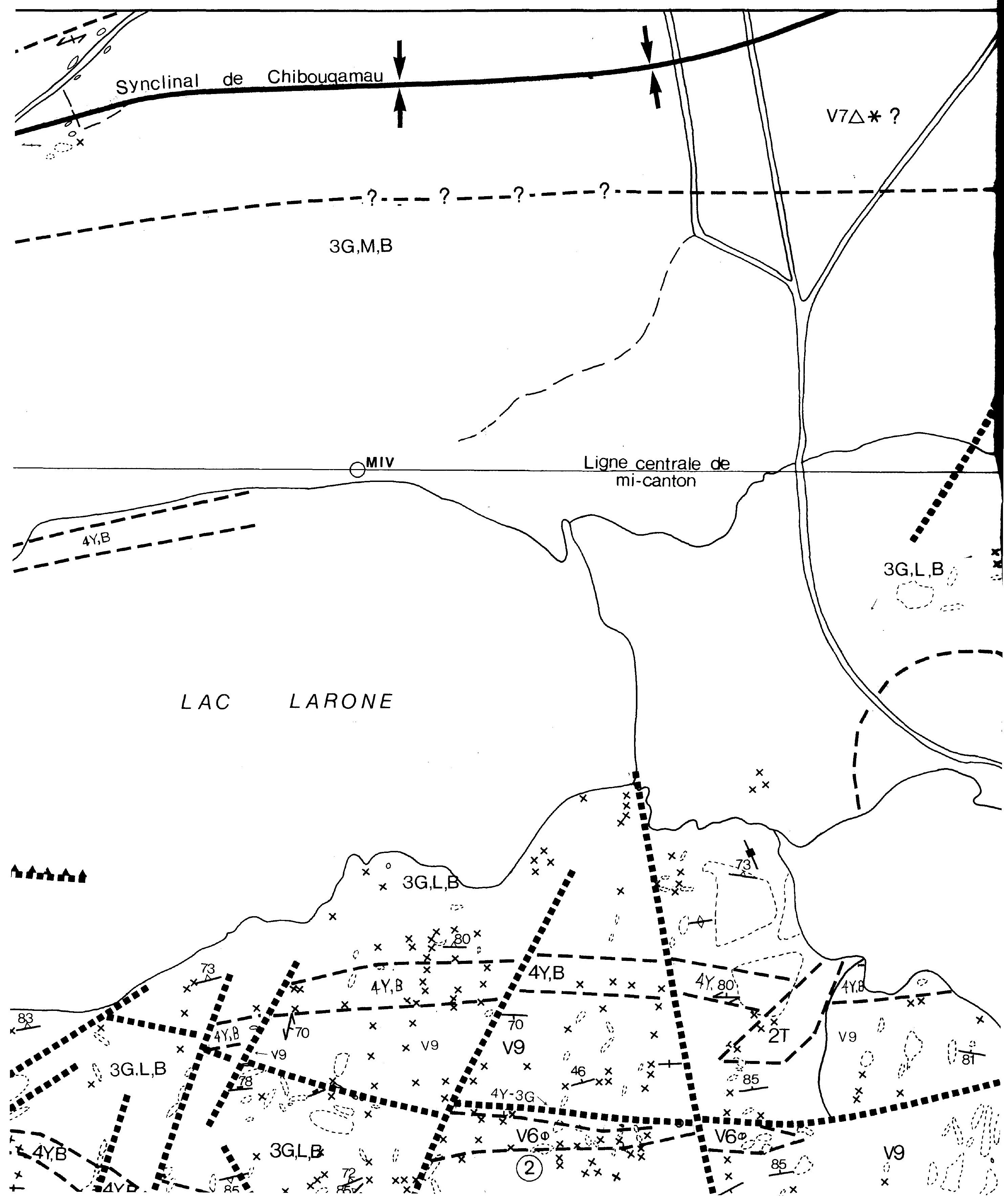




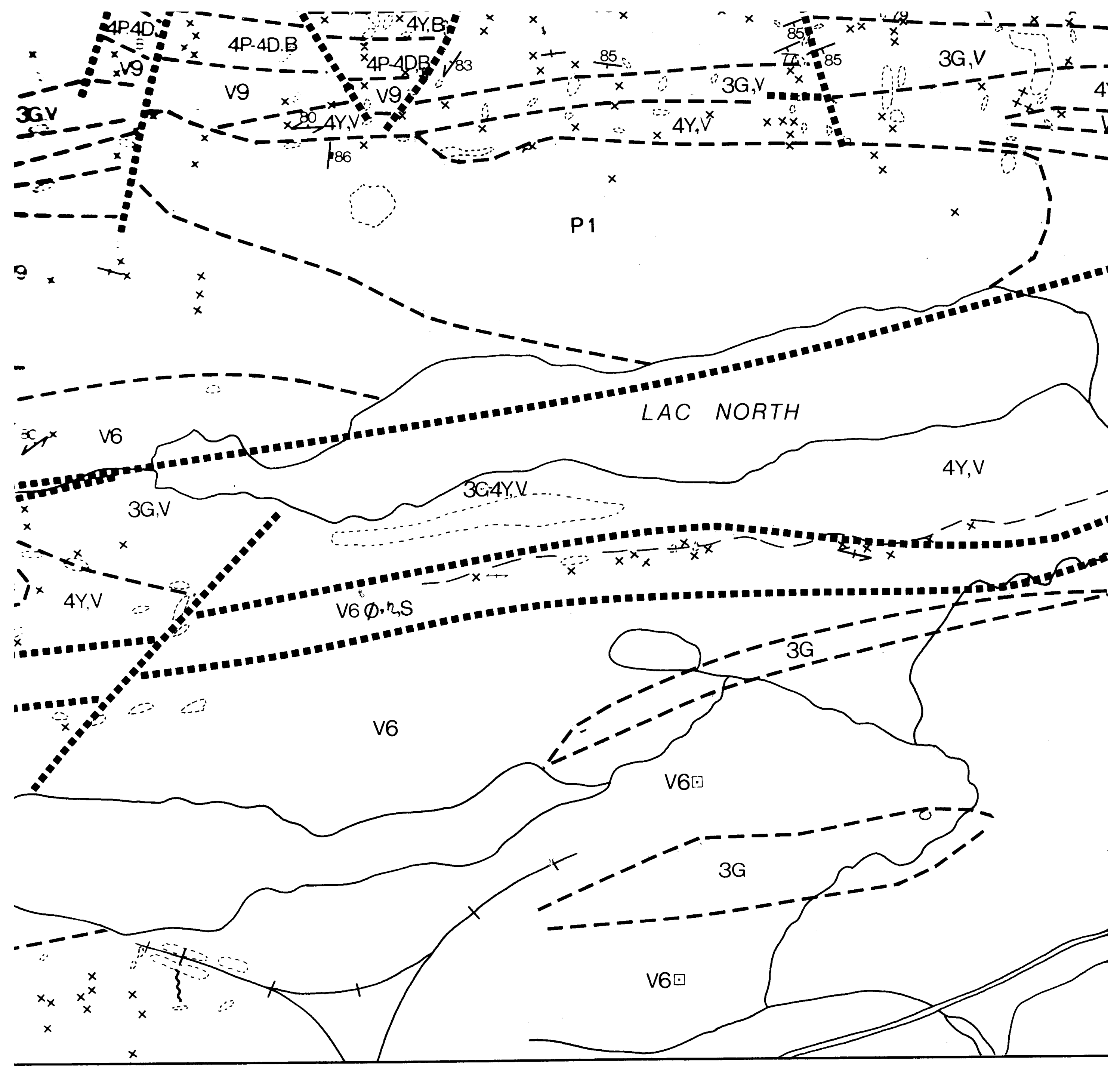




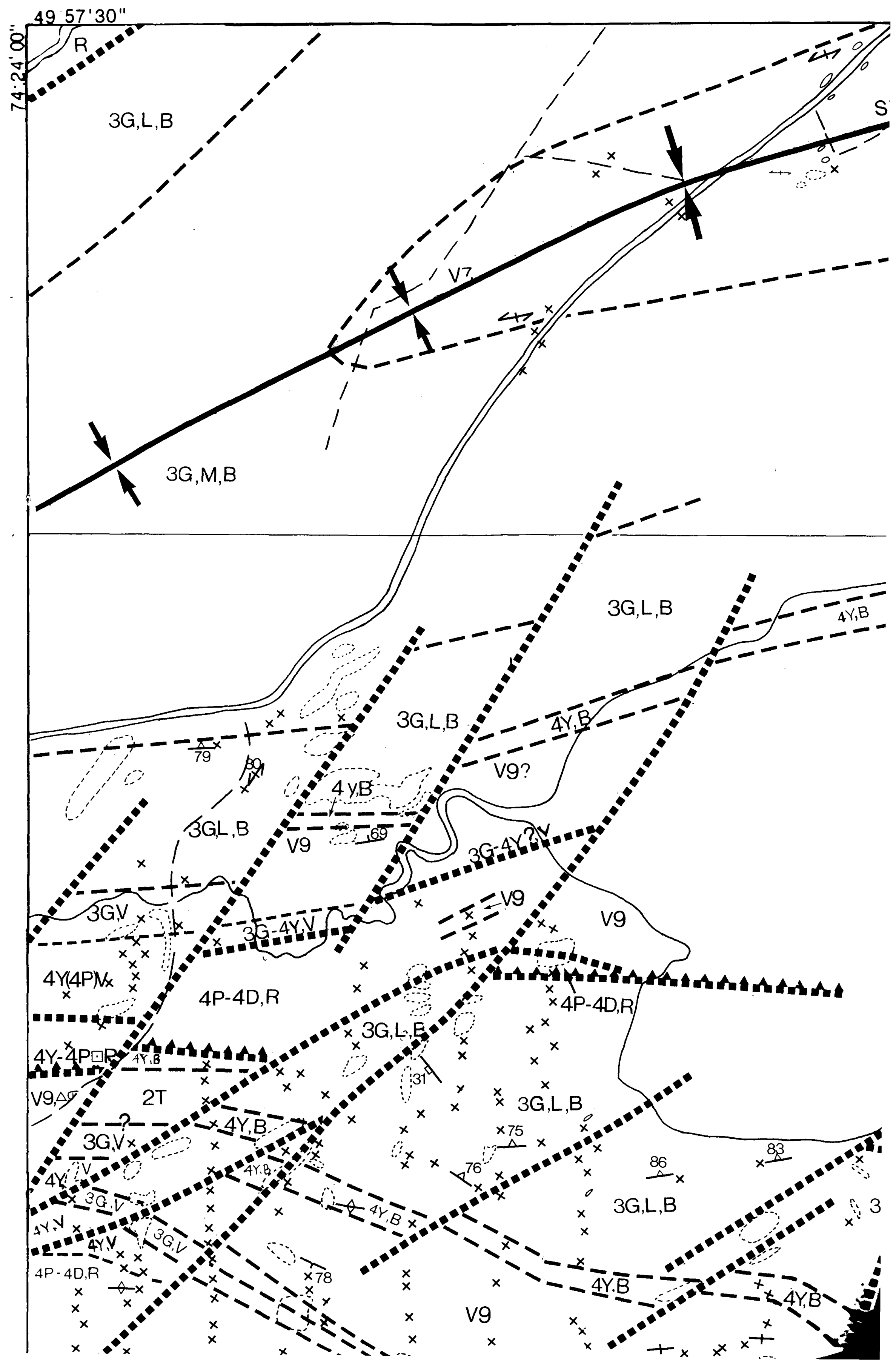




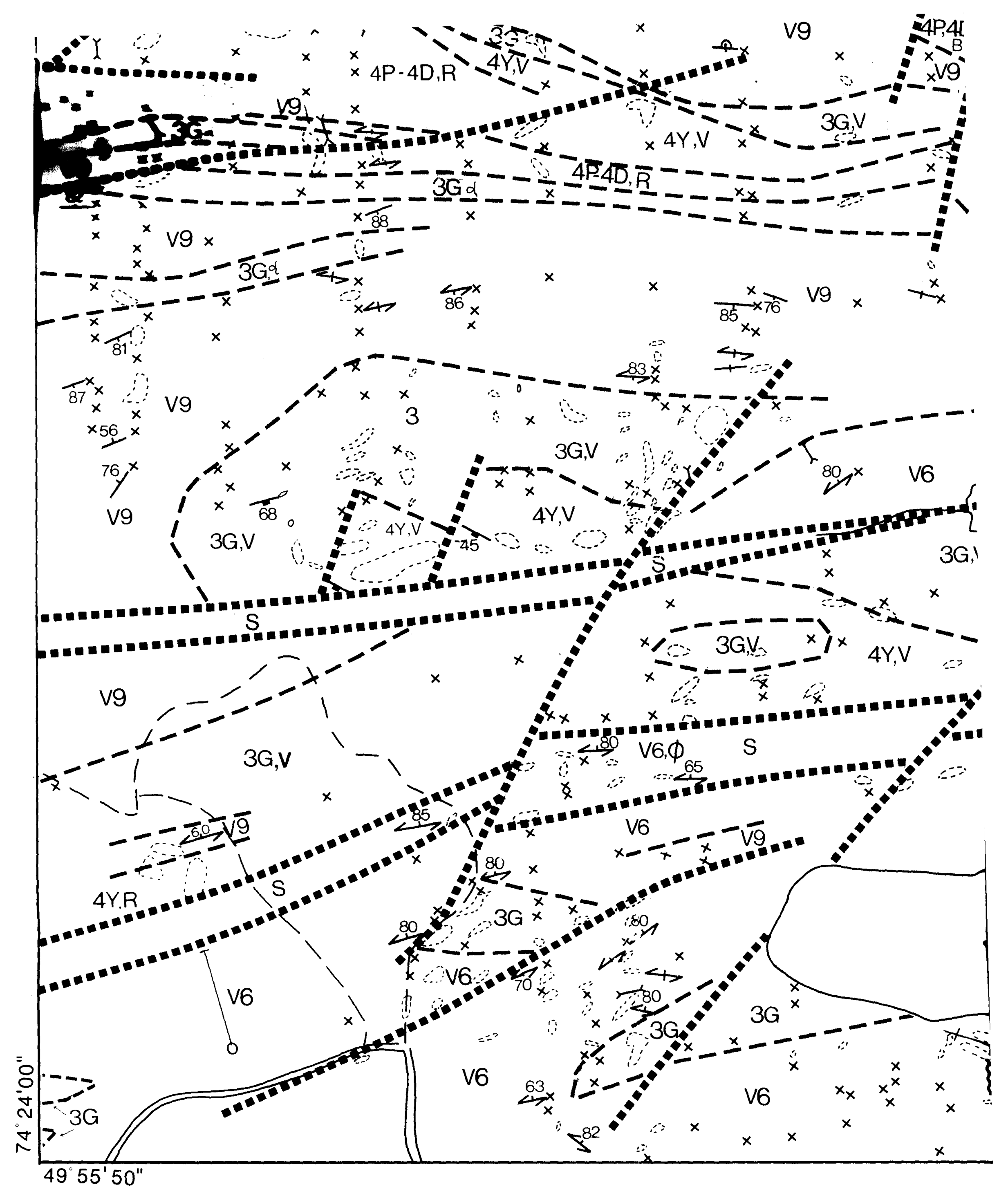

